\title{
Supproting Information
}

Preparation of $C_{2}$-Symmetric Bicyclo[2.2.2]octa-2,5-diene Ligands and Their Use for Rhodium-Catalyzed Asymmetric 1,4-Addition of Arylboronic Acids

Yusuke Otomaru, Kazuhiro Okamoto, Ryo Shintani, and Tamio Hayashi*

Department of Chemistry, Graduate School of Science, Kyoto University, Sakyo, Kyoto 606-8502, Japan

\section{Contents}

General and Materials

${ }^{1} \mathrm{H}$ NMR spectra for the compounds prepared

Data for X-ray structure
S14 
General. All anaerobic and moisture-sensitive manipulations were carried out with standard Schlenk technique under predried nitrogen or glovebox techniques under prepurified argon. NMR spectra were recorded at $500 \mathrm{MHz}$ for ${ }^{1} \mathrm{H}$ and 125 $\mathrm{MHz}$ for ${ }^{13} \mathrm{C}$. Chemical shifts are reported in $\mathrm{ppm}$ referenced to an internal $\mathrm{SiMe}_{4}$ standard for ${ }^{1} \mathrm{H}$ NMR, chloroform- $d(\square 77.05)$ for ${ }^{13} \mathrm{C}$ NMR. Chiralcel OJ (2 $\mathrm{cm} \square \times 25 \mathrm{~cm})$ was used for the separation of enantiomers of 2,5bis(trifluoromethanesulfonyloxy)bicyclo[2.2.2]octa-2,5-diene (2) and 2,5disubstituted bicyclo[2.2.2] octa-2,5-dienes (3a-d).

Materials. $\mathrm{THF}, \mathrm{Et}_{2} \mathrm{O}$, and dioxane were distilled from sodium benzophenoneketyl under nitrogen. Diisopropylamine was distilled from $\mathrm{CaH}_{2}$ under nitrogen. Racemic bicyclo[2.2.2] $\quad$ octane-2,5-dione $\quad(d l-1),{ }^{1} \quad$ (2-pyridyl)triflimide, ${ }^{2}$ $\mathrm{PdCl}_{2}(\mathrm{dppf}),{ }^{3}\left[\mathrm{RhCl}\left(\mathrm{C}_{2} \mathrm{H}_{4}\right)_{2}\right]_{2},{ }^{4}$ and $(1 R, 4 R)-2,5$-dibenzylbicylo[2.2.1]hepta-2,5diene $(7)^{5}$ were prepared according to the reported procedures.

(1) Werstiuk, N. H.; Yeroushalmi, S.; Guan-Lin, H. Can. J. Chem. 1992, 70, 974.

(2) Comins, D. L.; Dehghani, A. Tetrahedron Lett. 1992, 33, 6299.

(3) Hayashi, T.; Konishi, M.; Kobori, Y.; Kumada, M.; Higuchi, T.; Hirotsu, K. J. Am. Chem. Soc. 1984, 106, 158.

(4) Cramer, R. Inorg. Synth. 1974, 15, 14.

(5) Hayashi, T.; Ueyama, K.; Tokunaga, N.; Yoshida, K. J. Am. Chem. Soc. 2003, 125,11508 . 

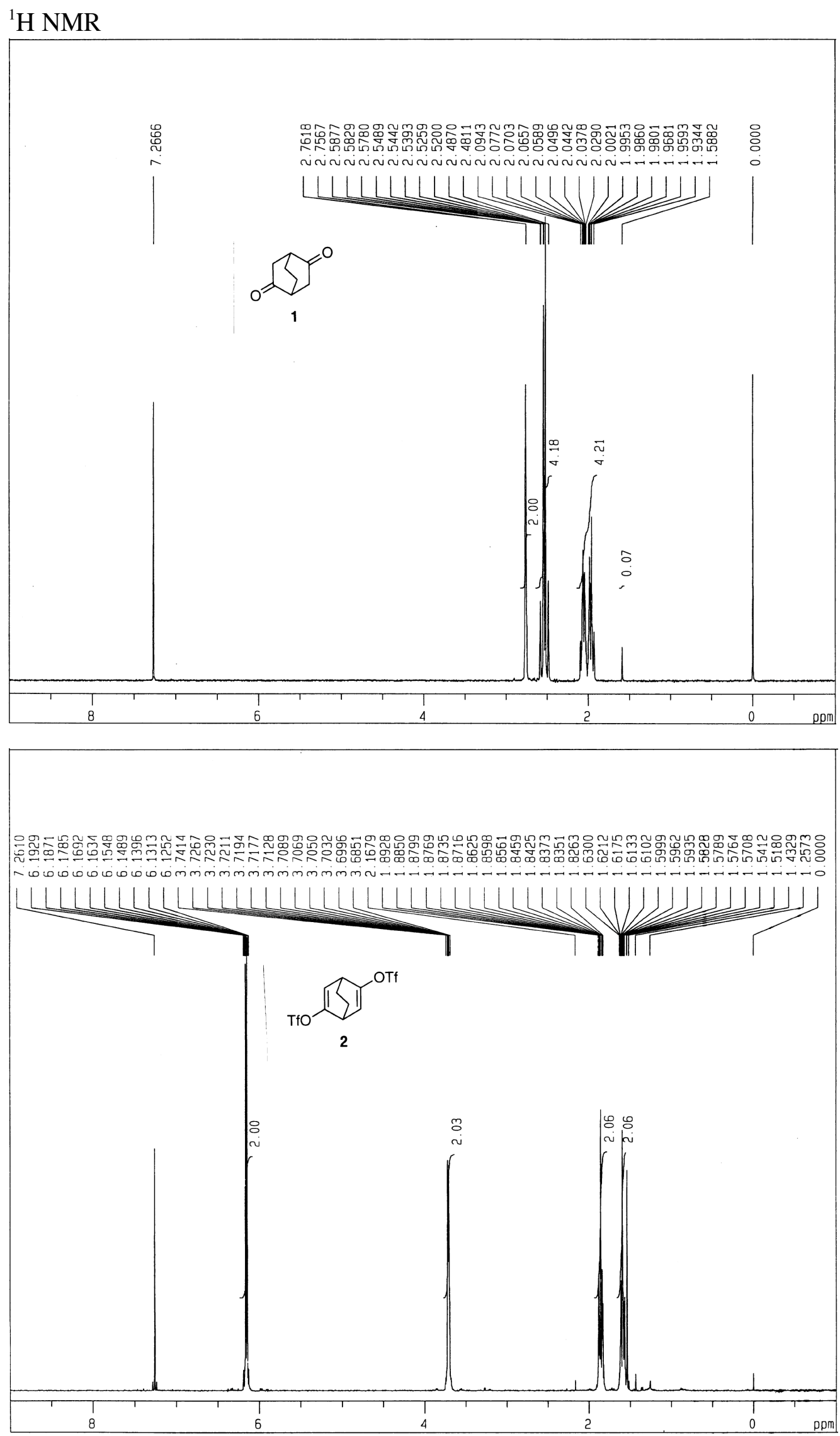

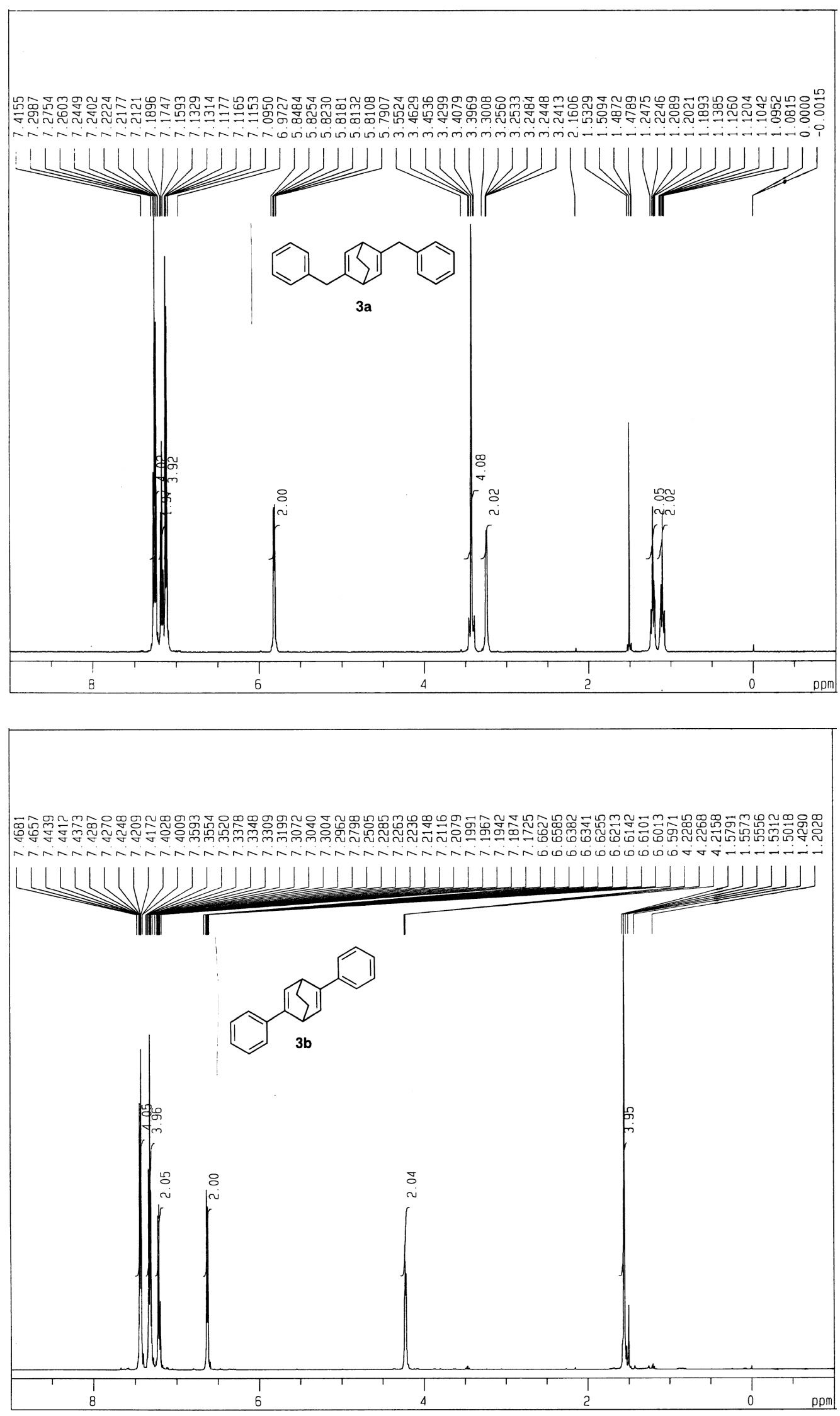

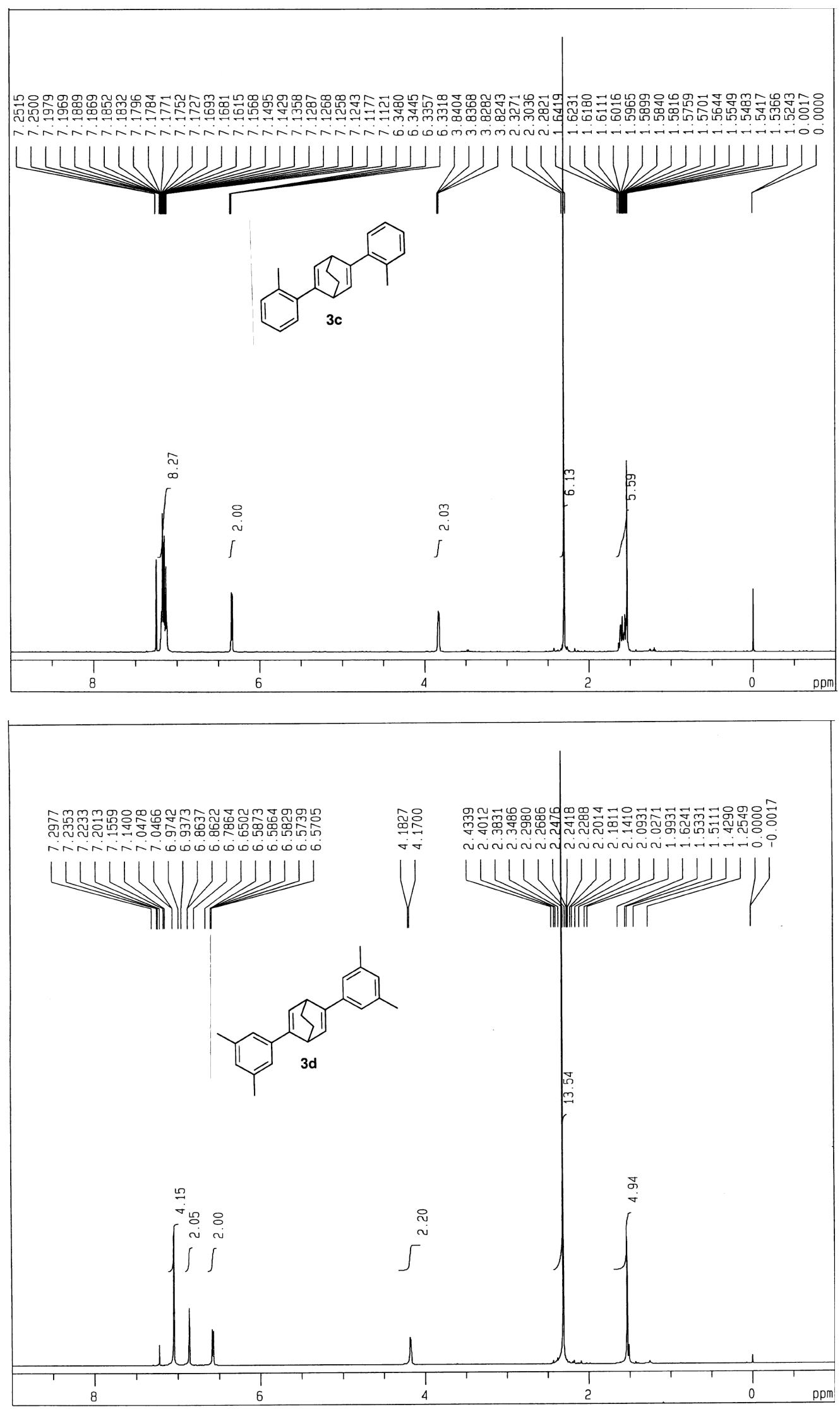

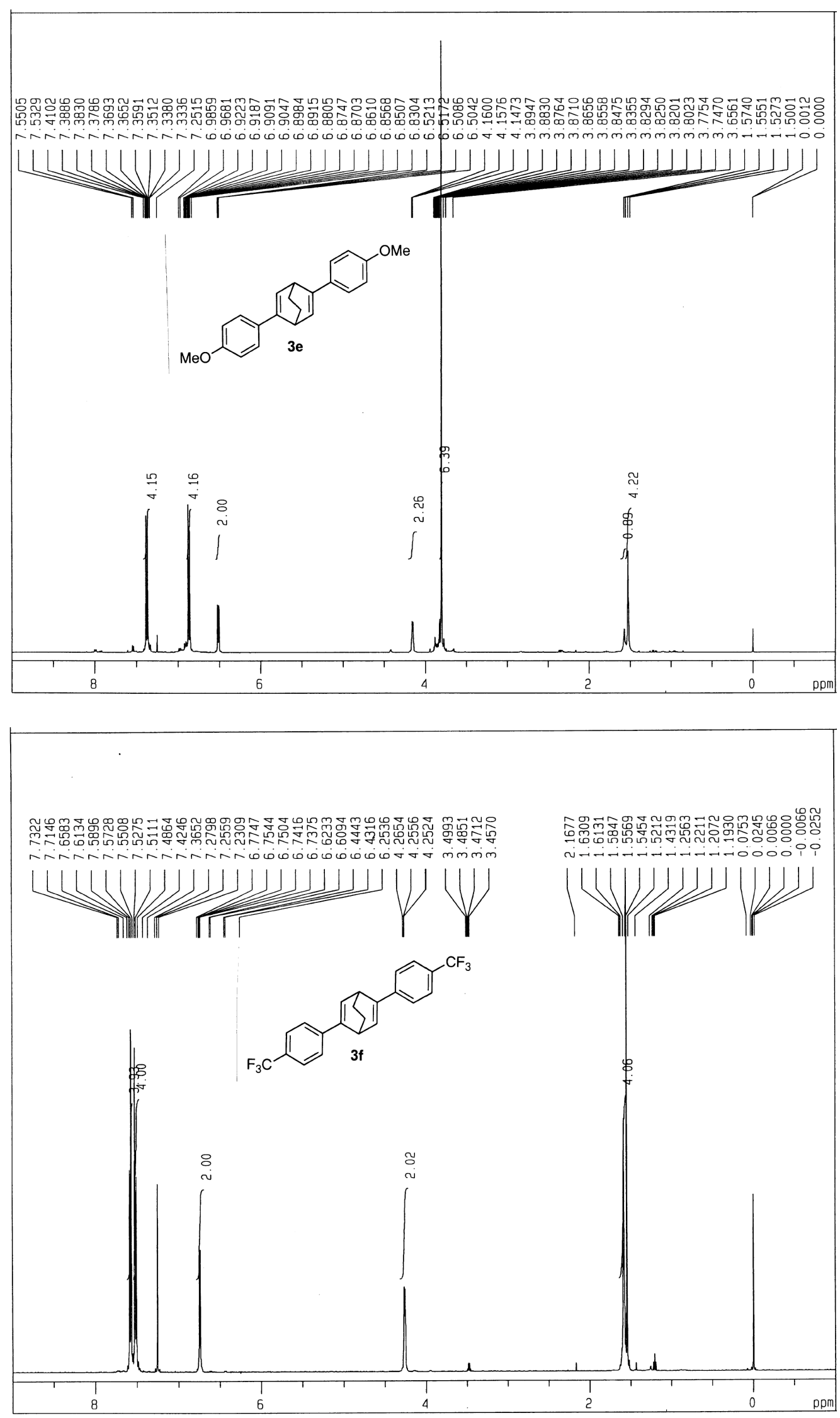

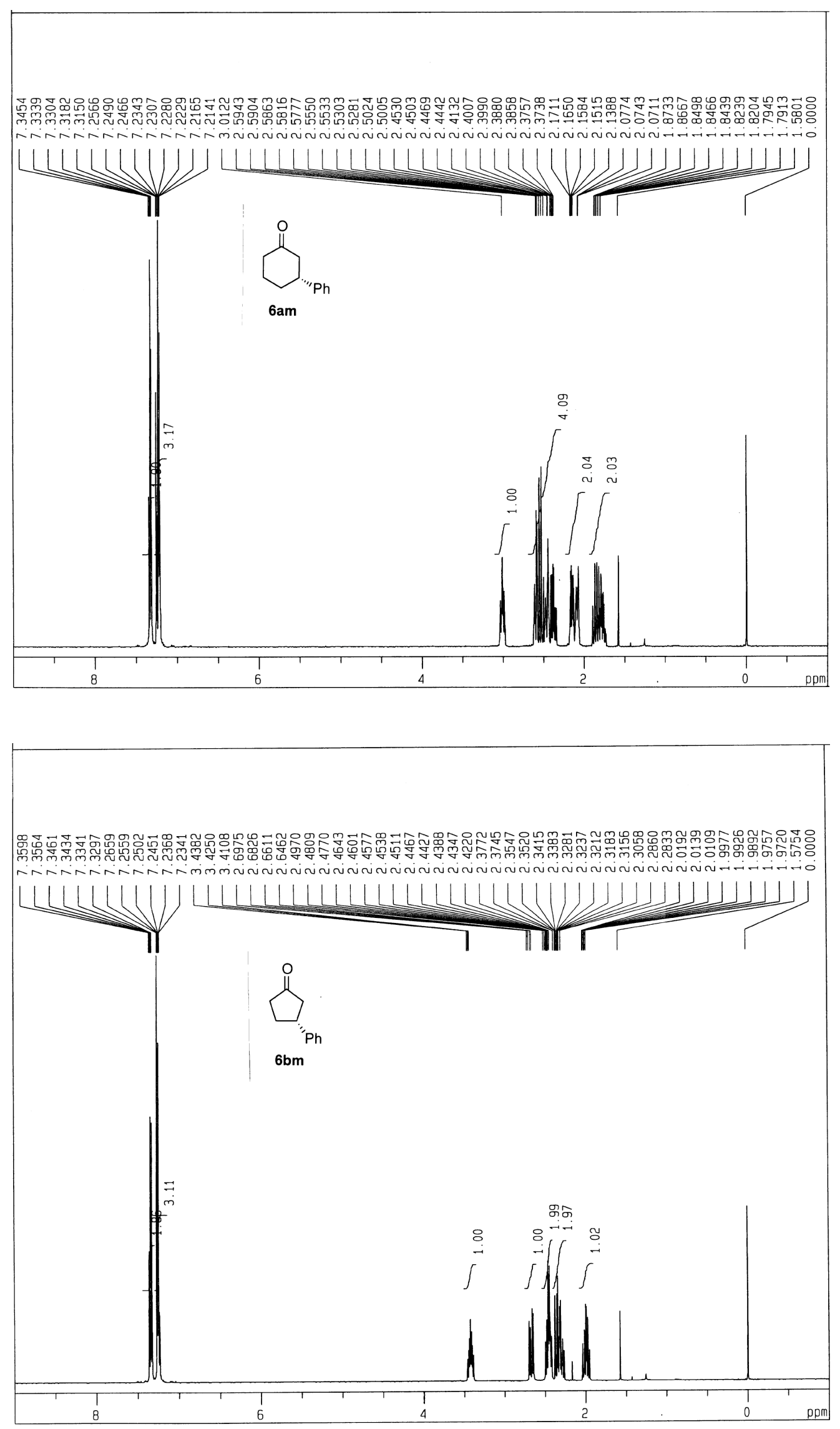

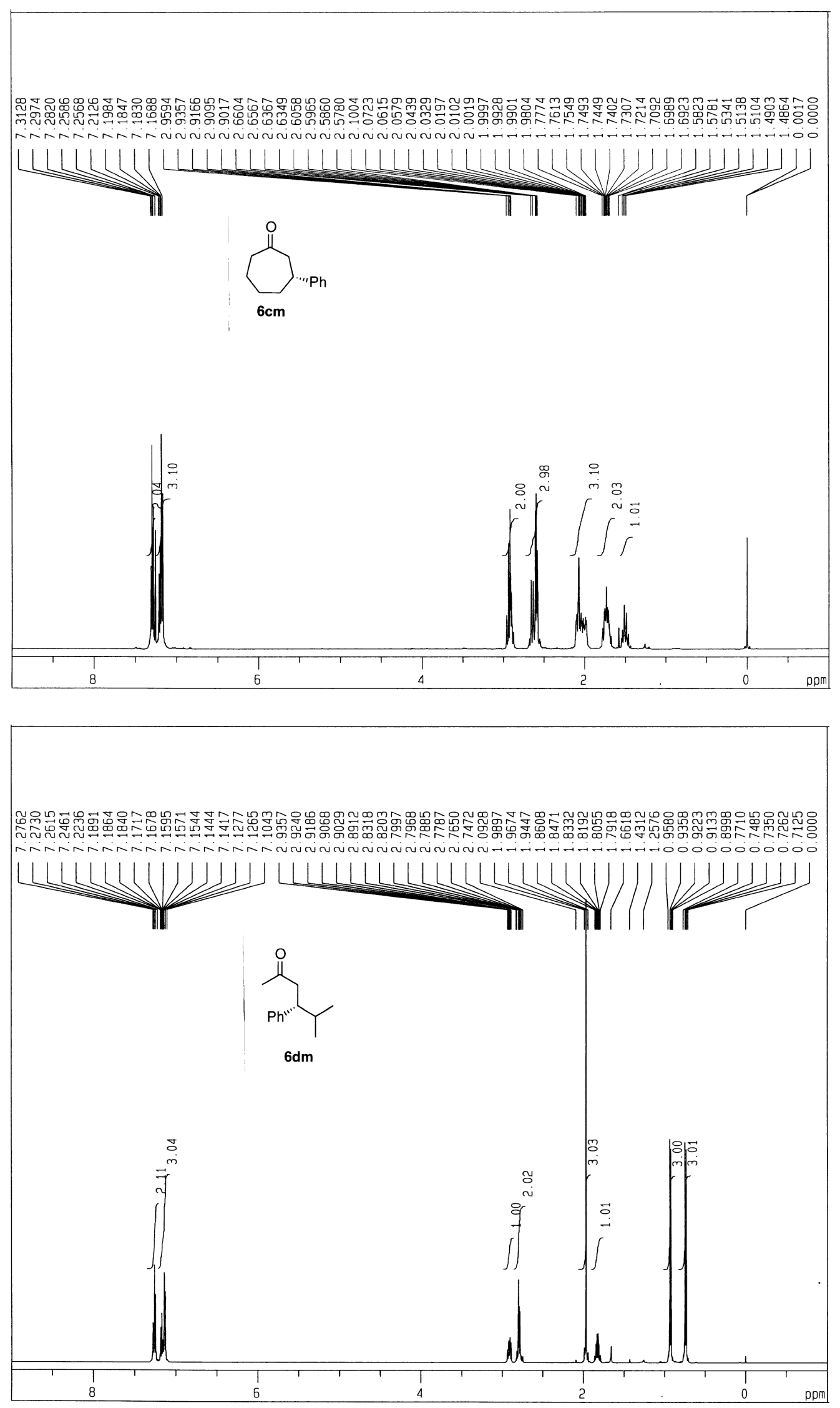

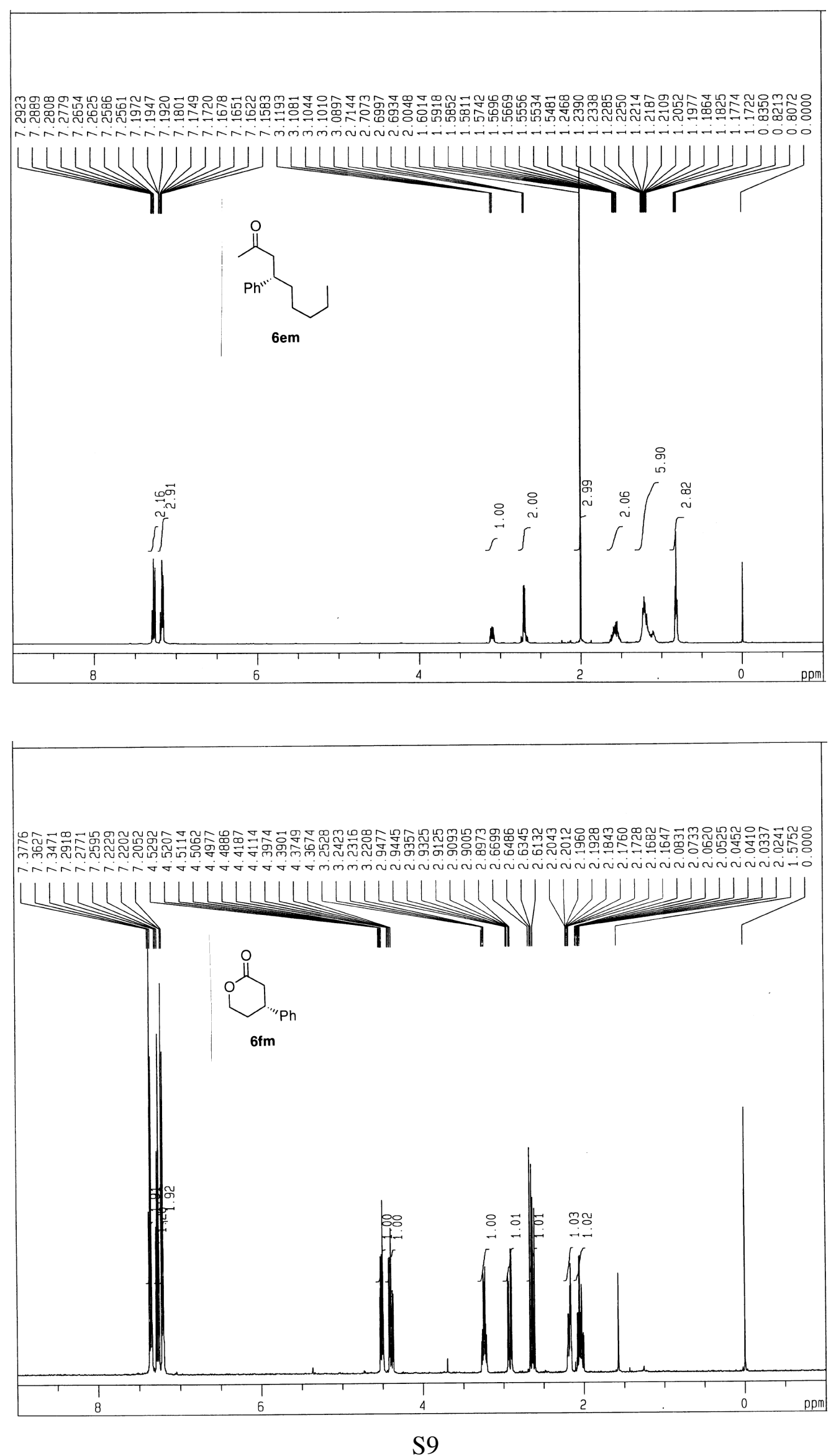

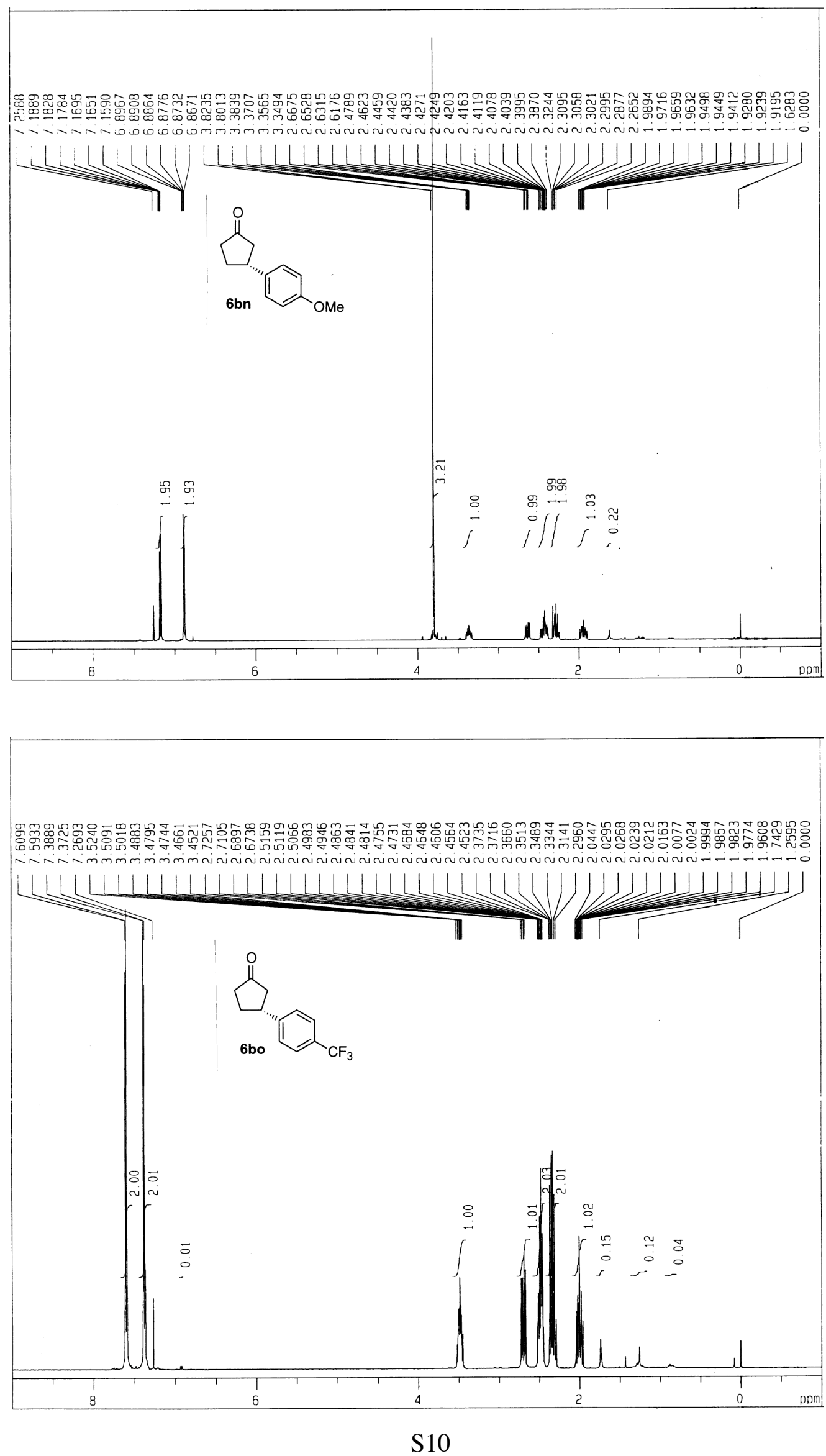

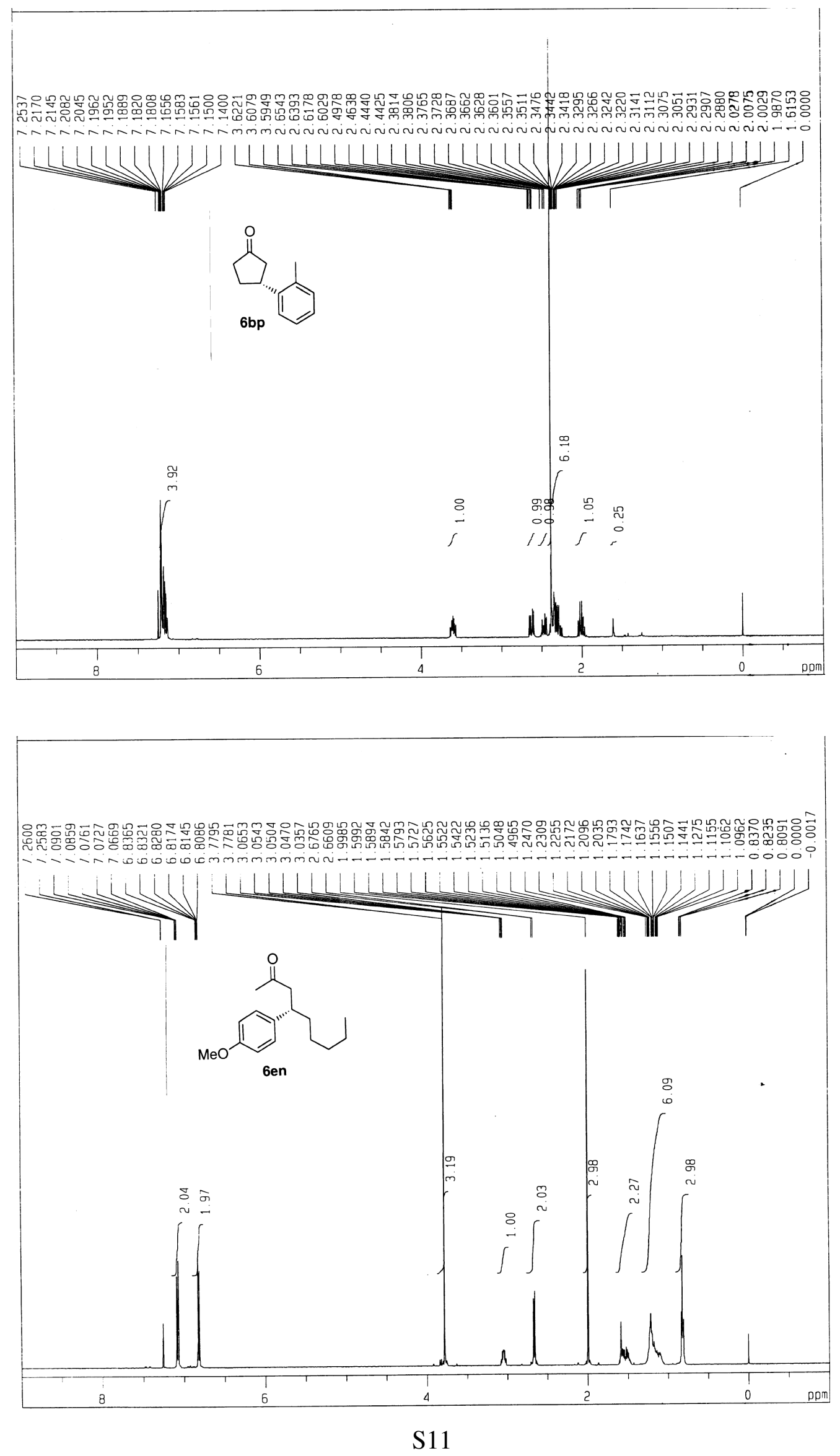

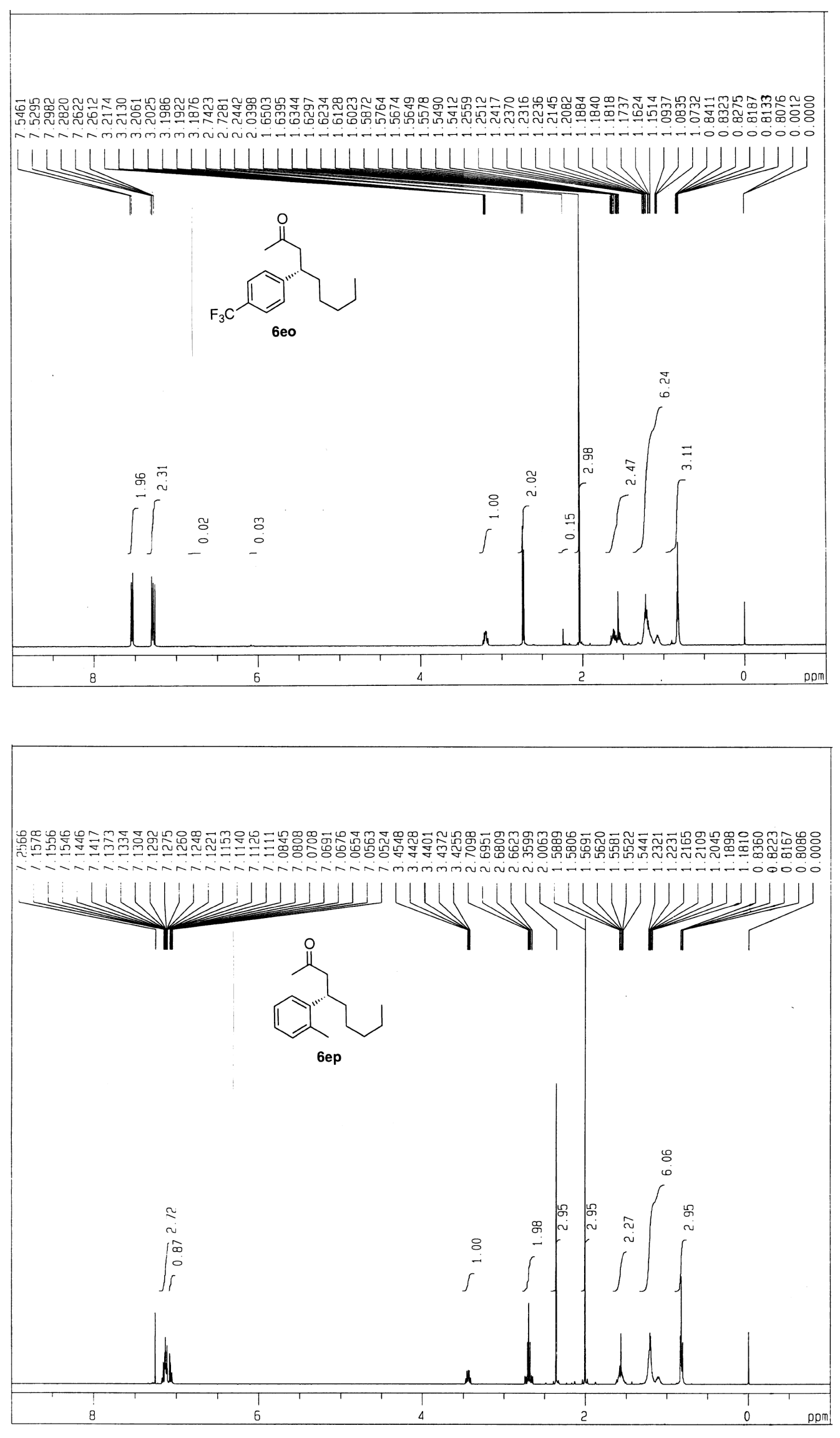

S12 


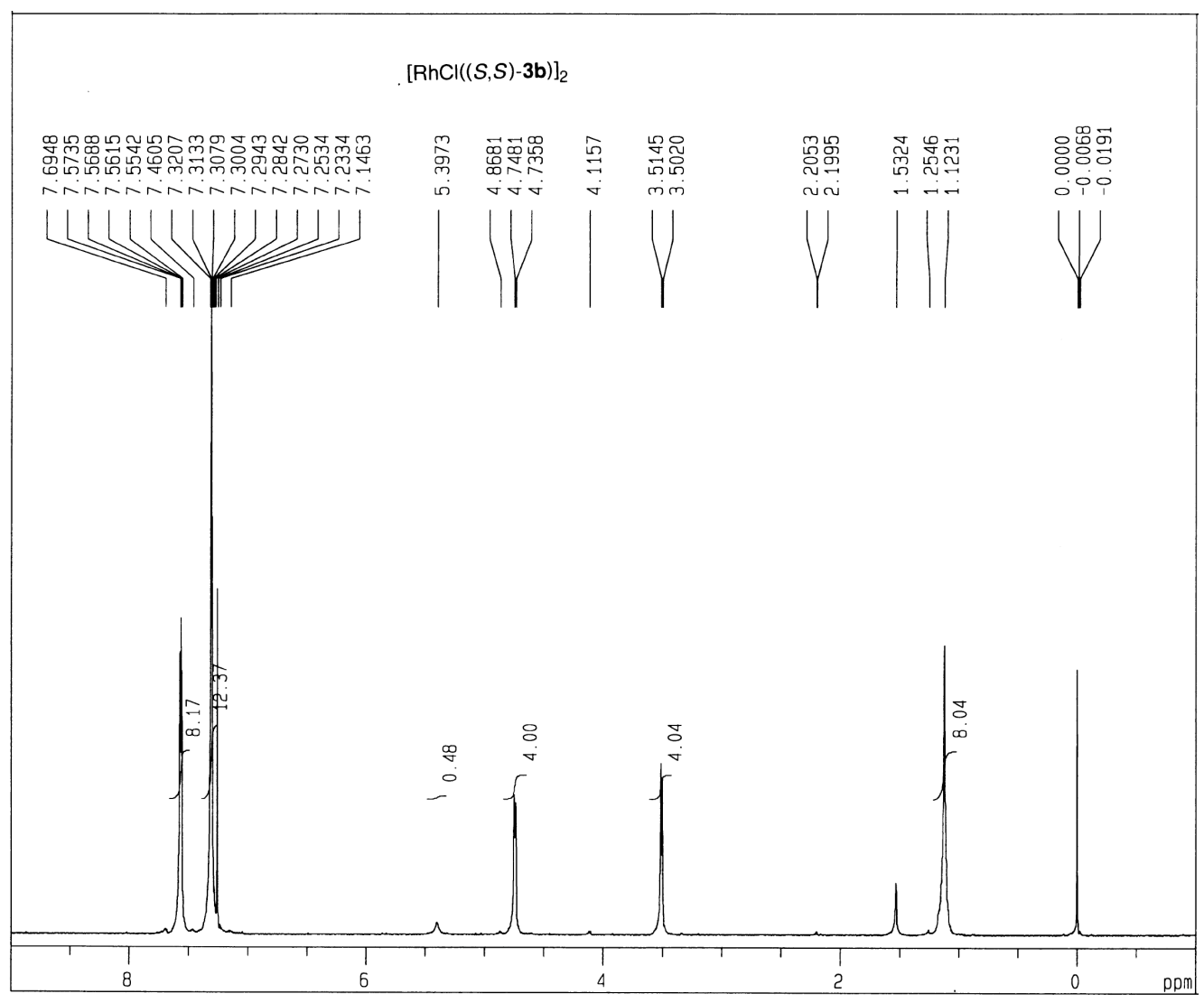




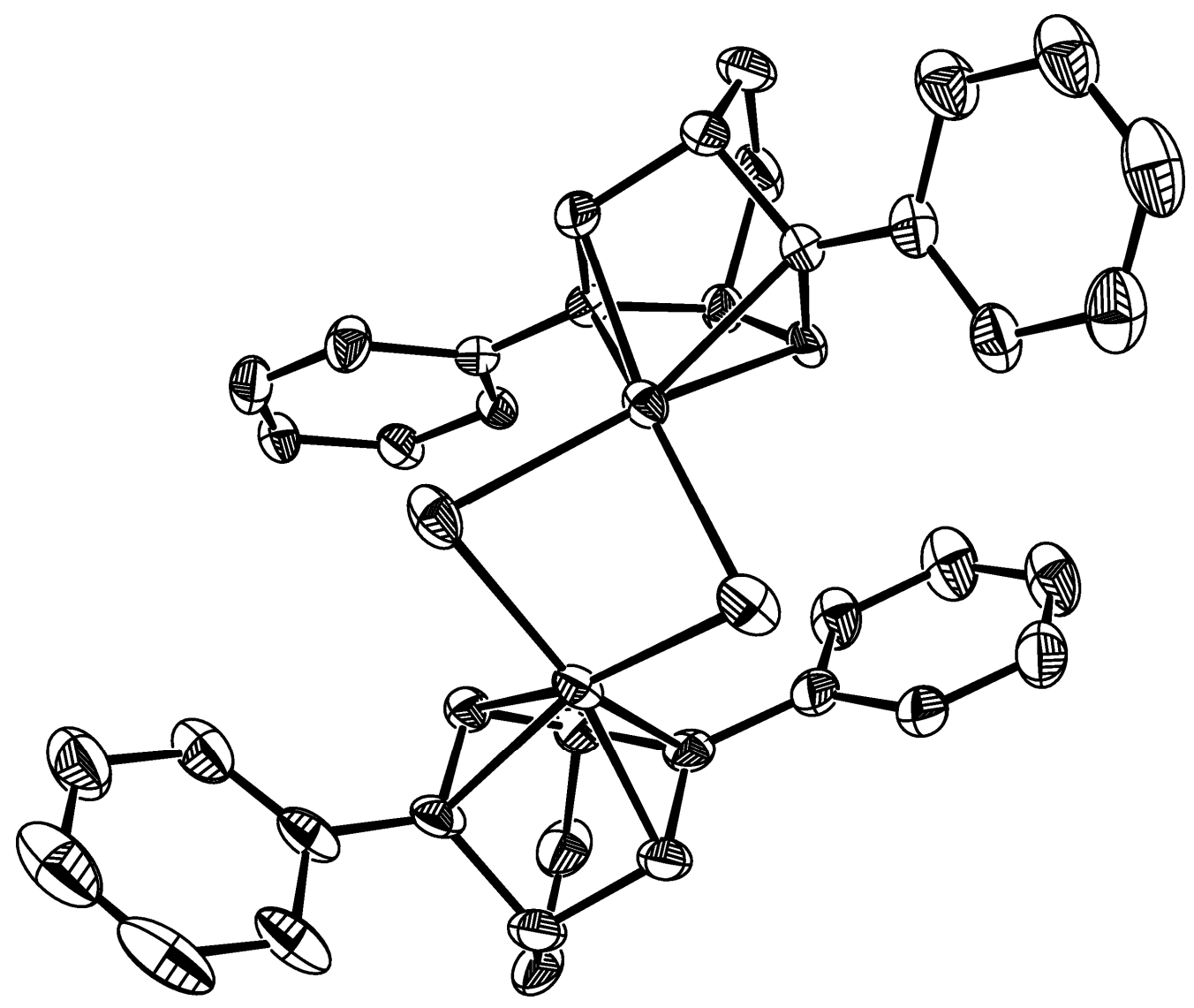

Table S1. Crystal Data

Empirical Formula

Formula Weight

Crystal Color, Habit

Crystal Dimensions

Crystal System

Lattice Type

Indexing Images

Detector Position

Pixel Size

Lattice Parameters

Space Group

$\mathrm{Z}$ value

$\mathrm{D}_{\text {calc }}$

$\mathrm{F}_{000}$

$\square(\mathrm{MoK} \square)$
$\mathrm{C}_{40} \mathrm{H}_{36} \mathrm{Rh}_{2} \mathrm{Cl}_{2}$

793.44

red, prism

$0.50 \times 0.40 \times 0.30 \mathrm{~mm}$

monoclinic

Primitive

3 oscillations at 30.0 seconds

$127.40 \mathrm{~mm}$

$0.100 \mathrm{~mm}$

$\mathrm{a}=13.45(1) \AA$

$\mathrm{b}=19.80(1) \AA$

$\mathrm{c}=18.97(1) \AA$

$\square=93.82(3)^{\circ}$

$\mathrm{V}=5039.6(6) \AA^{3}$

$\mathrm{P} 2{ }_{1}(\# 4)$

6

$1.569 \mathrm{~g} / \mathrm{cm}^{3}$

2400.00

$11.67 \mathrm{~cm}^{-1}$ 
Table S2. Intensity Measurements

Diffractometer

Radiation

Detector Aperture

Data Images

$\square$ oscillation Range $(\square=45.0, \square=0.0)$

Exposure Rate

$\square$ oscillation Range $(\square=45.0, \square=180.0)$

Exposure Rate

Detector Position

Pixel Size

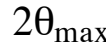

No. of Reflections Measured

Corrections

Table S3. Structure Solution and Refinement

Structure Solution

Refinement

Function Minimized

Least Squares Weights

Anomalous Dispersion

No. Observations $(\mathrm{I}>3.00 \square(\mathrm{I}))$

No. Variables

Reflection/Parameter Ratio

Residuals: R (I >3.00 $\square(\mathrm{I})$ )

Residuals: Rw (I>3.00 $\square(\mathrm{I})$ )

Goodness of Fit Indicator

Max Shift/Error in Final Cycle

Maximum peak in Final Diff. Map

Minimum peak in Final Diff. Map
Rigaku RAXIS-RAPID

$\operatorname{MoK} \square(\square=0.71075 \AA)$

graphite monochromated

$280 \mathrm{~mm} \times 256 \mathrm{~mm}$

74 exposures

$130.0-190.0^{\circ}$

$90.0 \mathrm{sec} . /^{\circ}$

$0.0-162.0^{\circ}$

$90.0 \mathrm{sec} . /^{\circ}$

$127.40 \mathrm{~mm}$

$0.100 \mathrm{~mm}$

$55.0^{\circ}$

Total: 48571

Unique: $11826\left(\mathrm{R}_{\mathrm{int}}=0.021\right)$

Lorentz-polarization

Direct Methods (SIR92)

Full-matrix least-squares on $\mathrm{F}$

$\square \mathrm{w}(|\mathrm{Fo}|-|\mathrm{Fc}|)^{2}$

All non-hydrogen atoms

10449

1298

8.05

0.029

0.043

2.12

0.00

$5.30 \mathrm{e}^{-} / \AA^{3}$

$-3.18 \mathrm{e}^{-} / \AA^{3}$

Table S4. Atomic coordinates and $\mathrm{B}_{\mathrm{iso}} / \mathrm{B}_{\mathrm{eq}}$

$\begin{array}{lcccc}\text { atom } & \mathrm{x} & \mathrm{y} & \mathrm{z} & \mathrm{B}_{\mathrm{eq}} \\ \operatorname{Rh}(5) & 0.32070(5) & -0.26760(7) & -1.53142(3) & 1.49(1) \\ \operatorname{Rh}(2) & -0.07854(5) & -0.39385(7) & -0.83460(4) & 1.83(1) \\ \operatorname{Rh}(3) & 0.42792(4) & -0.21864(7) & -1.12146(3) & 1.35(1) \\ \operatorname{Rh}(4) & 0.57482(4) & -0.32356(7) & -1.15821(3) & 1.25(1) \\ \operatorname{Rh}(6) & 0.17251(4) & -0.33263(7) & -1.44984(3) & 1.35(1) \\ \operatorname{Rh}(1) & -0.10882(5) & -0.25016(7) & -0.82333(3) & 1.67(1) \\ \operatorname{Cl}(2) & -0.2355(1) & -0.3354(1) & -0.8408(1) & 2.55(4) \\ \operatorname{Cl}(3) & 0.3974(1) & -0.3338(1) & -1.1558(1) & 1.73(3) \\ \operatorname{Cl}(4) & 0.5405(1) & -0.2183(1) & -1.2158(1) & 1.69(3)\end{array}$




\begin{tabular}{|c|c|c|c|c|}
\hline $\mathrm{Cl}(6)$ & $0.1503(1)$ & $-0.2336(1)$ & $-1.5224(1)$ & $1.84(4)$ \\
\hline $\mathrm{Cl}(5)$ & $0.2519(2)$ & $-0.3791(1)$ & $-1.5481(1)$ & $2.05(4)$ \\
\hline $\mathrm{Cl}(1)$ & $-0.0363(2)$ & $-0.3084(1)$ & $-0.9173(1)$ & $2.59(4)$ \\
\hline $\mathrm{C}(510)$ & $0.3070(7)$ & $-0.1329(5)$ & $-1.6762(5)$ & $2.6(2)$ \\
\hline $\mathrm{C}(303)$ & $0.4257(6)$ & $-0.1146(4)$ & $-1.0971(4)$ & $1.8(2)$ \\
\hline$C(102)$ & $-0.1744(6)$ & $-0.1690(4)$ & $-0.7651(4)$ & $1.9(2)$ \\
\hline$C(314)$ & $0.6491(7)$ & $-0.1127(5)$ & $-1.0975(5)$ & $2.4(2)$ \\
\hline$C(604)$ & $0.2291(6)$ & $-0.3496(4)$ & $-1.3104(4)$ & $1.7(1)$ \\
\hline C(309) & $0.6010(6)$ & $-0.1416(4)$ & $-1.0431(5)$ & $2.1(2)$ \\
\hline$C(403)$ & $0.6208(6)$ & $-0.4234(4)$ & $-1.1380(4)$ & $1.5(1)$ \\
\hline$C(213)$ & $-0.2787(8)$ & $-0.3706(6)$ & $-0.6294(6)$ & $3.2(2)$ \\
\hline$C(316)$ & $0.1517(6)$ & $-0.1972(4)$ & $-1.1641(4)$ & $1.8(2)$ \\
\hline $\mathrm{C}(401)$ & $0.7242(6)$ & $-0.3525(4)$ & $-1.0582(4)$ & $1.5(1)$ \\
\hline$C(405)$ & $0.7230(5)$ & $-0.3413(4)$ & $-1.1873(4)$ & $1.3(1)$ \\
\hline$C(104)$ & $-0.0265(6)$ & $-0.2110(4)$ & $-0.6952(4)$ & $1.8(2)$ \\
\hline$C(116)$ & $0.1685(6)$ & $-0.2789(4)$ & $-0.7158(4)$ & $1.8(2)$ \\
\hline C(109) & $-0.2810(6)$ & $-0.1512(5)$ & $-0.7752(4)$ & $2.2(2)$ \\
\hline$C(410)$ & $0.5568(7)$ & $-0.3580(6)$ & $-0.9600(5)$ & $3.1(2)$ \\
\hline$C(413)$ & $0.4104(7)$ & $-0.4561(5)$ & $-0.9736(6)$ & $2.9(2)$ \\
\hline C(616) & $0.3033(6)$ & $-0.2056(4)$ & $-1.3104(4)$ & $2.0(2)$ \\
\hline $\mathrm{C}(605)$ & $0.1720(6)$ & $-0.2913(4)$ & $-1.3453(4)$ & $1.5(1)$ \\
\hline $\mathrm{C}(408)$ & $0.8055(6)$ & $-0.4436(4)$ & $-1.1290(5)$ & $2.0(2)$ \\
\hline $\mathrm{C}(601)$ & $0.0514(6)$ & $-0.3852(4)$ & $-1.3555(4)$ & $2.0(2)$ \\
\hline C(306) & $0.3627(5)$ & $-0.2218(5)$ & $-1.0241(4)$ & $1.7(1)$ \\
\hline$C(417)$ & $0.7798(7)$ & $-0.3217(5)$ & $-1.3785(4)$ & $2.4(2)$ \\
\hline$C(406)$ & $0.7253(6)$ & $-0.3072(4)$ & $-1.1217(4)$ & $1.5(1)$ \\
\hline C(304) & $0.3176(6)$ & $-0.1137(4)$ & $-1.0795(5)$ & $2.0(2)$ \\
\hline $\mathrm{C}(114)$ & $-0.3565(7)$ & $-0.1993(5)$ & $-0.7734(5)$ & $2.7(2)$ \\
\hline C(318) & $0.0099(6)$ & $-0.2677(5)$ & $-1.1453(5)$ & $2.4(2)$ \\
\hline$C(503)$ & $0.3935(6)$ & $-0.1816(4)$ & $-1.4878(5)$ & $2.0(2)$ \\
\hline $\mathrm{C}(610)$ & $0.0146(6)$ & $-0.4988(5)$ & $-1.4637(4)$ & $2.1(2)$ \\
\hline$C(402)$ & $0.6242(6)$ & $-0.3883(4)$ & $-1.0727(4)$ & $1.5(1)$ \\
\hline$C(515)$ & $0.4865(6)$ & $-0.3495(4)$ & $-1.4492(5)$ & $1.9(2)$ \\
\hline$C(602)$ & $0.1319(6)$ & $-0.4207(4)$ & $-1.3945(4)$ & $1.6(1)$ \\
\hline$C(411)$ & $0.4881(8)$ & $-0.3676(8)$ & $-0.9078(6)$ & $5.0(3)$ \\
\hline $\mathrm{C}(517)$ & $0.5012(6)$ & $-0.4091(5)$ & $-1.3381(5)$ & $2.4(2)$ \\
\hline $\mathrm{C}(217)$ & $0.017(1)$ & $-0.5163(6)$ & $-1.0468(6)$ & $4.5(3)$ \\
\hline $\mathrm{C}(619)$ & $0.1783(7)$ & $-0.1001(4)$ & $-1.3517(5)$ & $2.4(2)$ \\
\hline C(418) & $0.8123(6)$ & $-0.2555(5)$ & $-1.3819(4)$ & $2.3(2)$ \\
\hline$C(416)$ & $0.7492(6)$ & $-0.3486(4)$ & $-1.3170(4)$ & $1.9(2)$ \\
\hline $\mathrm{C}(207)$ & $0.0803(8)$ & $-0.5115(5)$ & $-0.7051(6)$ & $3.2(2)$ \\
\hline $\mathrm{C}(613)$ & $0.1698(7)$ & $-0.5818(5)$ & $-1.4989(6)$ & $3.0(2)$ \\
\hline$C(618)$ & $0.2754(8)$ & $-0.0860(5)$ & $-1.3256(5)$ & $2.7(2)$ \\
\hline$C(310)$ & $0.6594(7)$ & $-0.1663(5)$ & $-0.9848(5)$ & $2.8(2)$ \\
\hline $\mathrm{C}(509)$ & $0.3242(6)$ & $-0.1266(4)$ & $-1.6031(5)$ & $2.1(2)$ \\
\hline C(103) & $-0.1360(6)$ & $-0.2206(4)$ & $-0.7200(4)$ & $1.7(1)$ \\
\hline $\mathrm{C}(311)$ & $0.7642(7)$ & $-0.1654(6)$ & $-0.9839(6)$ & $3.4(2)$ \\
\hline$C(305)$ & $0.2950(6)$ & $-0.1895(4)$ & $-1.0733(4)$ & $1.7(1)$ \\
\hline$C(206)$ & $0.0667(6)$ & $-0.4251(4)$ & $-0.8028(5)$ & $1.9(2)$ \\
\hline $\mathrm{C}(101)$ & $-0.0941(7)$ & $-0.1176(4)$ & $-0.7794(5)$ & $2.3(2)$ \\
\hline $\mathrm{C}(216)$ & $-0.0067(8)$ & $-0.5147(5)$ & $-0.9772(5)$ & $3.3(2)$ \\
\hline $\mathrm{C}(111)$ & $-0.4082(8)$ & $-0.0653(6)$ & $-0.7878(6)$ & $3.5(2)$ \\
\hline $\mathrm{C}(218)$ & $0.096(1)$ & $-0.4807(7)$ & $-1.0681(6)$ & $4.9(3)$ \\
\hline
\end{tabular}




\begin{tabular}{|c|c|c|c|c|}
\hline C(419) & $0.8139(6)$ & $-0.2173(5)$ & $-1.3215(5)$ & $2.4(2)$ \\
\hline C(308) & $0.3074(7)$ & $-0.0784(5)$ & $-1.0061(5)$ & $2.8(2)$ \\
\hline $\mathrm{C}(312)$ & $0.8104(7)$ & $-0.1366(5)$ & $-1.0403(6)$ & $3.1(2)$ \\
\hline C(118) & $0.3078(6)$ & $-0.3049(5)$ & $-0.7854(5)$ & $2.2(2)$ \\
\hline$C(603)$ & $0.2278(6)$ & $-0.3995(4)$ & $-1.3709(4)$ & $1.6(1)$ \\
\hline$C(507)$ & $0.5751(7)$ & $-0.1799(5)$ & $-1.5655(6)$ & $2.9(2)$ \\
\hline $\mathrm{C}(415)$ & $0.7524(5)$ & $-0.3111(4)$ & $-1.2551(4)$ & $1.5(1)$ \\
\hline $\mathrm{C}(620)$ & $0.1449(7)$ & $-0.1664(4)$ & $-1.3574(5)$ & $2.1(2)$ \\
\hline $\mathrm{C}(606)$ & $0.0749(6)$ & $-0.3113(4)$ & $-1.3704(4)$ & $1.8(2)$ \\
\hline$C(505)$ & $0.4716(6)$ & $-0.2862(4)$ & $-1.4907(5)$ & $1.9(2)$ \\
\hline $\mathrm{C}(210)$ & $-0.0716(8)$ & $-0.3755(5)$ & $-0.6154(5)$ & $2.7(2)$ \\
\hline C(108) & $-0.0115(7)$ & $-0.1410(5)$ & $-0.6598(5)$ & $2.8(2)$ \\
\hline $\mathrm{C}(412)$ & $0.4155(8)$ & $-0.4172(7)$ & $-0.9160(6)$ & $3.8(3)$ \\
\hline $\mathrm{C}(611)$ & $-0.0033(7)$ & $-0.5567(5)$ & $-1.5032(5)$ & $2.6(2)$ \\
\hline C(106) & $-0.0179(7)$ & $-0.1627(5)$ & $-0.8113(5)$ & $2.3(2)$ \\
\hline C(520) & $0.4983(9)$ & $-0.4123(5)$ & $-1.4823(5)$ & $3.1(2)$ \\
\hline C(113) & $-0.4564(8)$ & $-0.1789(6)$ & $-0.7788(5)$ & $3.4(2)$ \\
\hline $\mathrm{C}(211)$ & $-0.1238(8)$ & $-0.3436(6)$ & $-0.5649(5)$ & $3.5(2)$ \\
\hline C(105) & $0.0230(6)$ & $-0.2121(4)$ & $-0.7646(4)$ & 1.7(1) \\
\hline $\mathrm{C}(511)$ & $0.2454(8)$ & $-0.0858(5)$ & $-1.7144(6)$ & $3.3(2)$ \\
\hline$C(414)$ & $0.4790(7)$ & $-0.4483(5)$ & $-1.0244(5)$ & $2.5(2)$ \\
\hline C(203) & $-0.1093(7)$ & $-0.4832(4)$ & $-0.7813(6)$ & $2.6(2)$ \\
\hline $\mathrm{C}(315)$ & $0.1981(5)$ & $-0.2178(5)$ & $-1.0995(4)$ & $1.7(1)$ \\
\hline C(208) & $0.0327(8)$ & $-0.5636(5)$ & $-0.7593(7)$ & $3.7(2)$ \\
\hline C(301) & $0.4340(6)$ & $-0.1732(5)$ & $-0.9849(4)$ & $2.1(2)$ \\
\hline$C(215)$ & $0.0526(7)$ & $-0.4760(5)$ & $-0.9278(5)$ & $2.6(2)$ \\
\hline C(209) & $-0.1208(7)$ & $-0.4047(4)$ & $-0.6751(5)$ & $2.3(2)$ \\
\hline C(404) & $0.7169(6)$ & $-0.4179(4)$ & $-1.1765(4)$ & $1.5(1)$ \\
\hline C(407) & $0.8095(6)$ & $-0.4052(5)$ & $-1.0581(4)$ & $1.9(2)$ \\
\hline C(302) & $0.4908(6)$ & $-0.1467(4)$ & $-1.0460(4)$ & $1.8(2)$ \\
\hline C(313) & $0.7543(7)$ & $-0.1107(5)$ & $-1.0959(5)$ & $2.7(2)$ \\
\hline C(516) & $0.4901(6)$ & $-0.3499(5)$ & $-1.3745(5)$ & $2.1(2)$ \\
\hline $\mathrm{C}(202)$ & $-0.0671(6)$ & $-0.4373(4)$ & $-0.7311(5)$ & $2.1(2)$ \\
\hline$C(205)$ & $0.0256(6)$ & $-0.4714(4)$ & $-0.8525(5)$ & $2.1(2)$ \\
\hline $\mathrm{C}(120)$ & $0.1690(6)$ & $-0.2420(5)$ & $-0.8365(5)$ & $2.3(2)$ \\
\hline $\mathrm{C}(420)$ & $0.7846(6)$ & $-0.2446(4)$ & $-1.2586(4)$ & $2.0(2)$ \\
\hline $\mathrm{C}(614)$ & $0.1891(7)$ & $-0.5247(5)$ & $-1.4568(5)$ & $2.6(2)$ \\
\hline $\mathrm{C}(320)$ & $0.1490(6)$ & $-0.2638(5)$ & $-1.0584(4)$ & $2.4(2)$ \\
\hline C(319) & $0.0573(7)$ & $-0.2886(6)$ & $-1.0815(5)$ & $2.9(2)$ \\
\hline $\mathrm{C}(204)$ & $-0.0325(7)$ & $-0.5269(5)$ & $-0.8151(6)$ & $3.0(2)$ \\
\hline $\mathrm{C}(110)$ & $-0.3084(8)$ & $-0.0832(6)$ & $-0.7837(5)$ & $3.1(2)$ \\
\hline $\mathrm{C}(107)$ & $-0.0504(7)$ & $-0.0864(5)$ & $-0.7112(6)$ & $3.0(2)$ \\
\hline $\mathrm{C}(612)$ & $0.0726(7)$ & $-0.5979(5)$ & $-1.5211(5)$ & $2.6(2)$ \\
\hline $\mathrm{C}(617)$ & $0.3365(7)$ & $-0.1391(5)$ & $-1.3051(5)$ & $2.5(2)$ \\
\hline $\mathrm{C}(112)$ & $-0.4820(8)$ & $-0.1116(7)$ & $-0.7859(5)$ & $3.8(3)$ \\
\hline C(519) & $0.5073(9)$ & $-0.4714(5)$ & $-1.4440(6)$ & $3.5(2)$ \\
\hline $\mathrm{C}(317)$ & $0.0583(6)$ & $-0.2233(5)$ & $-1.1868(4)$ & $2.2(2)$ \\
\hline$C(607)$ & $0.0649(7)$ & $-0.3979(5)$ & $-1.2755(5)$ & $2.6(2)$ \\
\hline$C(115)$ & $0.1203(6)$ & $-0.2457(4)$ & $-0.7725(4)$ & $1.8(1)$ \\
\hline C(409) & $0.5526(6)$ & $-0.3985(4)$ & $-1.0183(4)$ & 1.7(1) \\
\hline $\mathrm{C}(514)$ & $0.2833(7)$ & $-0.0711(5)$ & $-1.5691(6)$ & $2.9(2)$ \\
\hline $\mathrm{C}(508)$ & $0.5820(7)$ & $-0.1848(5)$ & $-1.4839(6)$ & $3.0(2)$ \\
\hline$C(219)$ & $0.154(1)$ & $-0.4436(6)$ & $-1.0209(6)$ & $4.2(3)$ \\
\hline
\end{tabular}




\begin{tabular}{|c|c|c|c|c|}
\hline$C(502)$ & $0.3882(6)$ & $-0.1758(4)$ & $-1.5629(5)$ & $2.1(2)$ \\
\hline $\mathrm{C}(307)$ & $0.3788(7)$ & $-0.1149(5)$ & $-0.9512(5)$ & $2.5(2)$ \\
\hline C(615) & $0.2068(6)$ & $-0.2202(4)$ & $-1.3379(4)$ & $1.7(1)$ \\
\hline $\mathrm{C}(201)$ & $0.0471(6)$ & $-0.4399(5)$ & $-0.7273(5)$ & $2.1(2)$ \\
\hline C(119) & $0.2601(7)$ & $-0.2707(5)$ & $-0.8428(5)$ & $2.8(2)$ \\
\hline$C(506)$ & $0.4638(6)$ & $-0.2824(4)$ & $-1.5661(5)$ & $1.9(2)$ \\
\hline $\mathrm{C}(212)$ & $-0.2277(9)$ & $-0.3407(6)$ & $-0.5716(5)$ & $3.7(2)$ \\
\hline $\mathrm{C}(512)$ & $0.2065(7)$ & $-0.0307(5)$ & $-1.6804(6)$ & $3.3(2)$ \\
\hline $\mathrm{C}(214)$ & $-0.2264(7)$ & $-0.4019(5)$ & $-0.6800(6)$ & $2.8(2)$ \\
\hline C(608) & $0.1733(7)$ & $-0.3791(5)$ & $-1.2484(4)$ & $2.3(2)$ \\
\hline $\mathrm{C}(518)$ & $0.5107(7)$ & $-0.4705(5)$ & $-1.3703(5)$ & $2.6(2)$ \\
\hline $\mathrm{C}(117)$ & $0.2624(6)$ & $-0.3081(4)$ & $-0.7211(5)$ & $2.1(2)$ \\
\hline $\mathrm{C}(501)$ & $0.4751(6)$ & $-0.2116(4)$ & $-1.5949(5)$ & $2.2(2)$ \\
\hline $\mathrm{C}(504)$ & $0.4866(6)$ & $-0.2172(5)$ & $-1.4574(5)$ & $2.2(2)$ \\
\hline$C(220)$ & $0.1324(8)$ & $-0.4412(5)$ & $-0.9509(5)$ & $3.2(2)$ \\
\hline C(609) & $0.1116(6)$ & $-0.4815(4)$ & $-1.4390(4)$ & $1.5(1)$ \\
\hline $\mathrm{C}(513)$ & $0.2254(7)$ & $-0.0245(5)$ & $-1.6070(6)$ & $3.3(2)$ \\
\hline $\mathrm{H}(511)$ & $0.1670(7)$ & $0.0016(5)$ & $-1.7065(6)$ & $3.9(3)$ \\
\hline $\mathrm{H}(512)$ & $0.1980(7)$ & $0.0127(5)$ & $-1.5833(6)$ & $4.1(3)$ \\
\hline $\mathrm{H}(509)$ & $0.3364(7)$ & $-0.1691(5)$ & $-1.7002(5)$ & $3.1(2)$ \\
\hline $\mathrm{H}(510)$ & $0.2317(8)$ & $-0.0914(5)$ & $-1.7638(6)$ & $3.9(2)$ \\
\hline $\mathrm{H}(513)$ & $0.2960(7)$ & $-0.0659(5)$ & $-1.5196(6)$ & $3.6(2)$ \\
\hline $\mathrm{H}(515)$ & $0.5026(6)$ & $-0.4077(5)$ & $-1.2880(5)$ & $2.8(2)$ \\
\hline $\mathrm{H}(518)$ & $0.4996(9)$ & $-0.4139(5)$ & $-1.5322(5)$ & $3.7(2)$ \\
\hline $\mathrm{H}(514)$ & $0.4853(6)$ & $-0.3086(5)$ & $-1.3495(5)$ & $2.5(2)$ \\
\hline $\mathrm{H}(517)$ & $0.5114(9)$ & $-0.5133(5)$ & $-1.4680(6)$ & $4.2(3)$ \\
\hline $\mathrm{H}(516)$ & $0.5191(7)$ & $-0.5108(5)$ & $-1.3434(5)$ & $3.1(2)$ \\
\hline $\mathrm{H}(609)$ & $-0.0394(6)$ & $-0.4704(5)$ & $-1.4529(4)$ & $2.5(2)$ \\
\hline $\mathrm{H}(612)$ & $0.2230(7)$ & $-0.6096(5)$ & $-1.5119(6)$ & $3.6(2)$ \\
\hline $\mathrm{H}(610)$ & $-0.0700(7)$ & $-0.5680(5)$ & $-1.5184(5)$ & $3.1(2)$ \\
\hline $\mathrm{H}(613)$ & $0.2555(7)$ & $-0.5145(5)$ & $-1.4401(5)$ & $3.1(2)$ \\
\hline $\mathrm{H}(611)$ & $0.0585(7)$ & $-0.6373(5)$ & $-1.5485(5)$ & $3.1(2)$ \\
\hline $\mathrm{H}(614)$ & $0.3468(6)$ & $-0.2413(4)$ & $-1.2953(4)$ & $2.4(2)$ \\
\hline $\mathrm{H}(617)$ & $0.1345(7)$ & $-0.0640(4)$ & $-1.3652(5)$ & $2.8(2)$ \\
\hline $\mathrm{H}(616)$ & $0.2995(8)$ & $-0.0410(5)$ & $-1.3225(5)$ & $3.2(2)$ \\
\hline H(618) & $0.0786(7)$ & $-0.1752(4)$ & $-1.3756(5)$ & $2.5(2)$ \\
\hline $\mathrm{H}(615)$ & $0.4025(7)$ & $-0.1303(5)$ & $-1.2864(5)$ & $3.0(2)$ \\
\hline $\mathrm{H}(501)$ & $0.4684(6)$ & $-0.2108(4)$ & $-1.6451(5)$ & $2.7(2)$ \\
\hline $\mathrm{H}(506)$ & $0.5770(7)$ & $-0.1338(5)$ & $-1.5793(6)$ & $3.5(2)$ \\
\hline $\mathrm{H}(505)$ & $0.6294(7)$ & $-0.2033(5)$ & $-1.5838(6)$ & $3.5(2)$ \\
\hline $\mathrm{H}(507)$ & $0.6379(7)$ & $-0.2118(5)$ & $-1.4690(6)$ & $3.6(2)$ \\
\hline $\mathrm{H}(508)$ & $0.5900(7)$ & $-0.1408(5)$ & $-1.4644(6)$ & $3.6(2)$ \\
\hline $\mathrm{H}(503)$ & $0.4887(6)$ & $-0.2198(5)$ & $-1.4074(5)$ & $2.7(2)$ \\
\hline $\mathrm{H}(605)$ & $0.0189(7)$ & $-0.3709(5)$ & $-1.2520(5)$ & $3.2(2)$ \\
\hline $\mathrm{H}(606)$ & $0.0531(7)$ & $-0.4442(5)$ & $-1.2661(5)$ & $3.2(2)$ \\
\hline $\mathrm{H}(608)$ & $0.2068(7)$ & $-0.4183(5)$ & $-1.2304(4)$ & $2.8(2)$ \\
\hline $\mathrm{H}(607)$ & $0.1714(7)$ & $-0.3463(5)$ & $-1.2121(4)$ & $2.8(2)$ \\
\hline $\mathrm{H}(603)$ & $0.2953(6)$ & $-0.3366(4)$ & $-1.2957(4)$ & $2.0(2)$ \\
\hline $\mathrm{H}(601)$ & $-0.0134(6)$ & $-0.3974(4)$ & $-1.3742(4)$ & $2.4(2)$ \\
\hline $\mathrm{H}(602)$ & $0.2839(6)$ & $-0.4271(4)$ & $-1.3782(4)$ & $1.9(2)$ \\
\hline $\mathrm{H}(604)$ & $0.0230(6)$ & $-0.2790(4)$ & $-1.3781(4)$ & $2.2(2)$ \\
\hline $\mathrm{H}(502)$ & $0.3589(6)$ & $-0.1506(4)$ & $-1.4601(5)$ & $2.4(2)$ \\
\hline $\mathrm{H}(504)$ & $0.4779(6)$ & $-0.3208(4)$ & $-1.5937(5)$ & $2.4(2)$ \\
\hline
\end{tabular}




\begin{tabular}{|c|c|c|c|c|}
\hline $\mathrm{H}(313)$ & $0.6106(7)$ & $-0.0938(5)$ & $-1.1364(5)$ & $2.9(2)$ \\
\hline H(309) & $0.6278(7)$ & $-0.1842(5)$ & $-0.9455(5)$ & $3.4(2)$ \\
\hline $\mathrm{H}(310)$ & $0.8034(7)$ & $-0.1837(6)$ & $-0.9449(6)$ & $4.0(2)$ \\
\hline $\mathrm{H}(311)$ & $0.8811(7)$ & $-0.1351(5)$ & $-1.0394(6)$ & $3.7(2)$ \\
\hline $\mathrm{H}(312)$ & $0.7863(7)$ & $-0.0917(5)$ & $-1.1344(5)$ & $3.2(2)$ \\
\hline $\mathrm{H}(314)$ & $0.1838(6)$ & $-0.1657(4)$ & $-1.1928(4)$ & $2.2(2)$ \\
\hline $\mathrm{H}(316)$ & $-0.0549(6)$ & $-0.2836(5)$ & $-1.1599(5)$ & $2.9(2)$ \\
\hline H(318) & $0.1796(6)$ & $-0.2779(5)$ & $-1.0143(4)$ & $2.8(2)$ \\
\hline $\mathrm{H}(317)$ & $0.0258(7)$ & $-0.3209(6)$ & $-1.0534(5)$ & $3.5(2)$ \\
\hline $\mathrm{H}(315)$ & $0.0281(6)$ & $-0.2102(5)$ & $-1.2314(4)$ & $2.6(2)$ \\
\hline H(409) & $0.6055(7)$ & $-0.3233(6)$ & $-0.9551(5)$ & $3.8(2)$ \\
\hline $\mathrm{H}(412)$ & $0.3586(7)$ & $-0.4886(5)$ & $-0.9805(6)$ & $3.5(2)$ \\
\hline $\mathrm{H}(410)$ & $0.4910(8)$ & $-0.3399(8)$ & $-0.8668(6)$ & $6.0(4)$ \\
\hline $\mathrm{H}(411)$ & $0.3706(8)$ & $-0.4247(7)$ & $-0.8802(6)$ & $4.7(3)$ \\
\hline $\mathrm{H}(413)$ & $0.4762(7)$ & $-0.4776(5)$ & $-1.0642(5)$ & $3.0(2)$ \\
\hline $\mathrm{H}(415)$ & $0.7788(7)$ & $-0.3493(5)$ & $-1.4196(4)$ & $2.9(2)$ \\
\hline $\mathrm{H}(416)$ & $0.8328(6)$ & $-0.2371(5)$ & $-1.4249(4)$ & $2.7(2)$ \\
\hline $\mathrm{H}(414)$ & $0.7248(6)$ & $-0.3936(4)$ & $-1.3166(4)$ & $2.3(2)$ \\
\hline $\mathrm{H}(417)$ & $0.8360(6)$ & $-0.1717(5)$ & $-1.3225(5)$ & $2.9(2)$ \\
\hline $\mathrm{H}(418)$ & $0.7859(6)$ & $-0.2173(4)$ & $-1.2173(4)$ & $2.4(2)$ \\
\hline H(308) & $0.3253(7)$ & $-0.0321(5)$ & $-1.0092(5)$ & $3.3(2)$ \\
\hline H(307) & $0.2407(7)$ & $-0.0818(5)$ & $-0.9929(5)$ & $3.3(2)$ \\
\hline $\mathrm{H}(306)$ & $0.4261(7)$ & $-0.0832(5)$ & $-0.9318(5)$ & $3.0(2)$ \\
\hline H(305) & $0.3410(7)$ & $-0.1322(5)$ & $-0.9147(5)$ & $3.0(2)$ \\
\hline $\mathrm{H}(301)$ & $0.4772(6)$ & $-0.1958(5)$ & $-0.9510(4)$ & $2.5(2)$ \\
\hline $\mathrm{H}(303)$ & $0.2760(6)$ & $-0.0930(4)$ & $-1.1158(5)$ & $2.4(2)$ \\
\hline $\mathrm{H}(304)$ & $0.3460(5)$ & $-0.2638(5)$ & $-1.0036(4)$ & $2.0(2)$ \\
\hline $\mathrm{H}(302)$ & $0.4502(6)$ & $-0.0822(4)$ & $-1.1285(4)$ & $2.1(2)$ \\
\hline $\mathrm{H}(408)$ & $0.7981(6)$ & $-0.4906(4)$ & $-1.1203(5)$ & $2.4(2)$ \\
\hline $\mathrm{H}(407)$ & $0.8654(6)$ & $-0.4362(4)$ & $-1.1517(5)$ & $2.4(2)$ \\
\hline $\mathrm{H}(405)$ & $0.8716(6)$ & $-0.3824(5)$ & $-1.0516(4)$ & $2.3(2)$ \\
\hline $\mathrm{H}(406)$ & $0.8031(6)$ & $-0.4363(5)$ & $-1.0205(4)$ & $2.3(2)$ \\
\hline $\mathrm{H}(404)$ & $0.7511(6)$ & $-0.2626(4)$ & $-1.1176(4)$ & $1.8(2)$ \\
\hline $\mathrm{H}(403)$ & $0.7114(6)$ & $-0.4411(4)$ & $-1.2204(4)$ & $1.8(2)$ \\
\hline $\mathrm{H}(401)$ & $0.7258(6)$ & $-0.3273(4)$ & $-1.0156(4)$ & $1.8(2)$ \\
\hline $\mathrm{H}(402)$ & $0.5759(6)$ & $-0.4599(4)$ & $-1.1467(4)$ & $1.8(2)$ \\
\hline $\mathrm{H}(215)$ & $-0.022(1)$ & $-0.5423(6)$ & $-1.0807(6)$ & $5.3(3)$ \\
\hline $\mathrm{H}(214)$ & $-0.0622(8)$ & $-0.5395(5)$ & $-0.9624(5)$ & $3.9(3)$ \\
\hline $\mathrm{H}(216)$ & $0.111(1)$ & $-0.4809(7)$ & $-1.1163(6)$ & $5.9(4)$ \\
\hline $\mathrm{H}(217)$ & $0.211(1)$ & $-0.4202(6)$ & $-1.0354(6)$ & $5.0(3)$ \\
\hline $\mathrm{H}(218)$ & $0.1721(8)$ & $-0.4149(5)$ & $-0.9180(5)$ & $3.8(2)$ \\
\hline $\mathrm{H}(212)$ & $-0.3494(8)$ & $-0.3697(6)$ & $-0.6341(6)$ & $3.9(2)$ \\
\hline $\mathrm{H}(209)$ & $-0.0010(8)$ & $-0.3787(5)$ & $-0.6094(5)$ & $3.3(2)$ \\
\hline $\mathrm{H}(210)$ & $-0.0884(8)$ & $-0.3234(6)$ & $-0.5253(5)$ & $4.2(3)$ \\
\hline $\mathrm{H}(211)$ & $-0.2632(9)$ & $-0.3180(6)$ & $-0.5370(5)$ & $4.5(3)$ \\
\hline $\mathrm{H}(213)$ & $-0.2615(7)$ & $-0.4221(5)$ & $-0.7197(6)$ & $3.4(2)$ \\
\hline H(206) & $0.0583(8)$ & $-0.5211(5)$ & $-0.6596(6)$ & $3.8(2)$ \\
\hline $\mathrm{H}(205)$ & $0.1509(8)$ & $-0.5146(5)$ & $-0.7037(6)$ & $3.8(2)$ \\
\hline $\mathrm{H}(202)$ & $-0.1760(7)$ & $-0.4984(4)$ & $-0.7787(6)$ & $3.1(2)$ \\
\hline $\mathrm{H}(207)$ & $0.0844(8)$ & $-0.5869(5)$ & $-0.7809(7)$ & $4.5(3)$ \\
\hline H(208) & $-0.0066(8)$ & $-0.5950(5)$ & $-0.7355(7)$ & $4.5(3)$ \\
\hline $\mathrm{H}(203)$ & $-0.0628(7)$ & $-0.5576(5)$ & $-0.8485(6)$ & $3.7(2)$ \\
\hline $\mathrm{H}(204)$ & $0.1223(6)$ & $-0.3983(4)$ & $-0.8137(5)$ & $2.3(2)$ \\
\hline
\end{tabular}




$\begin{array}{lrrrr}\mathrm{H}(201) & 0.0752(6) & -0.4065(5) & -0.6960(5) & 2.6(2) \\ \mathrm{H}(114) & 0.1373(6) & -0.2816(4) & -0.6724(4) & 2.2(2) \\ \mathrm{H}(116) & 0.3705(6) & -0.3260(5) & -0.7899(5) & 2.7(2) \\ \mathrm{H}(118) & 0.1371(6) & -0.2190(5) & -0.8756(5) & 2.8(2) \\ \mathrm{H}(117) & 0.2910(7) & -0.2675(5) & -0.8862(5) & 3.4(2) \\ \mathrm{H}(115) & 0.2951(6) & -0.3297(4) & -0.6813(5) & 2.5(2) \\ \mathrm{H}(113) & -0.3400(7) & -0.2458(5) & -0.7690(5) & 3.3(2) \\ \mathrm{H}(110) & -0.4253(8) & -0.0188(6) & -0.7921(6) & 4.2(3) \\ \mathrm{H}(112) & -0.5075(8) & -0.2118(6) & -0.7767(5) & 4.1(3) \\ \mathrm{H}(109) & -0.2584(8) & -0.0495(6) & -0.7861(5) & 3.8(2) \\ \mathrm{H}(111) & -0.5500(8) & -0.0982(7) & -0.7902(5) & 4.5(3) \\ \mathrm{H}(103) & -0.0029(6) & -0.2467(4) & -0.6651(4) & 2.2(2) \\ \mathrm{H}(101) & -0.1185(7) & -0.0842(4) & -0.8121(5) & 2.8(2) \\ \mathrm{H}(108) & -0.0469(7) & -0.1391(5) & -0.6182(5) & 3.3(2) \\ \mathrm{H}(107) & 0.0575(7) & -0.1338(5) & -0.6480(5) & 3.3(2) \\ \mathrm{H}(105) & 0.0029(7) & -0.0574(5) & -0.7218(6) & 3.7(2) \\ \mathrm{H}(106) & -0.1005(7) & -0.0609(5) & -0.6902(6) & 3.7(2) \\ \mathrm{H}(104) & 0.0187(7) & -0.1472(5) & -0.8493(5) & 2.8(2) \\ \mathrm{H}(102) & -0.1792(6) & -0.2464(4) & -0.6929(4) & 2.0(2)\end{array}$

$\mathrm{B}_{\mathrm{eq}}=8 / 3 \square^{2}\left(\mathrm{U}_{11}\left(\mathrm{aa}^{\star}\right)^{2}+\mathrm{U}_{22}\left(\mathrm{bb}^{\star}\right)^{2}+\mathrm{U}_{33}\left(\mathrm{cc}^{\star}\right)^{2}+2 \mathrm{U}_{12}\left(\mathrm{aa}^{\star} \mathrm{b} \mathrm{b}^{\star}\right) \cos \square+2 \mathrm{U}_{13}\left(\mathrm{aa}^{\star} \mathrm{cc}^{\star}\right) \cos \square+2 \mathrm{U}_{23}\left(\mathrm{bb}^{\star} \mathrm{cc}^{\star}\right) \cos \square\right)$

Table S5. Anisotropic Displacement Parameters

$\begin{array}{llllllc}\text { atom } & \mathrm{U}_{11} & \mathrm{U}_{22} & \mathrm{U}_{33} & \mathrm{U}_{12} & \mathrm{U}_{13} & \mathrm{U}_{23} \\ \mathrm{Rh}(5) & 0.0200(3) & 0.0161(3) & 0.0205(3) & 0.0000(2) & 0.0029(2) & 0.0005(2) \\ \mathrm{Rh}(2) & 0.0235(3) & 0.0196(3) & 0.0261(3) & 0.0022(3) & -0.0009(3) & -0.0072(3) \\ \mathrm{Rh}(3) & 0.0150(3) & 0.0178(3) & 0.0189(3) & 0.0013(2) & 0.0020(2) & 0.0022(2) \\ \mathrm{Rh}(4) & 0.0150(3) & 0.0155(3) & 0.0169(3) & 0.0011(2) & 0.0010(2) & -0.0000(2) \\ \mathrm{Rh}(6) & 0.0154(3) & 0.0169(3) & 0.0187(3) & -0.0002(2) & 0.0003(2) & -0.0003(2) \\ \mathrm{Rh}(1) & 0.0263(3) & 0.0207(3) & 0.0165(3) & 0.0052(3) & 0.0024(2) & 0.0008(2) \\ \mathrm{Cl}(2) & 0.024(1) & 0.034(1) & 0.037(1) & 0.0051(9) & -0.0070(8) & -0.011(1) \\ \mathrm{Cl}(3) & 0.0170(8) & 0.0212(9) & 0.0275(9) & -0.0005(7) & -0.0003(7) & 0.0002(8) \\ \mathrm{Cl}(4) & 0.0203(8) & 0.0214(9) & 0.0231(8) & 0.0032(8) & 0.0047(7) & 0.0052(8) \\ \mathrm{Cl}(6) & 0.0220(9) & 0.023(1) & 0.0245(9) & 0.0029(7) & -0.0011(7) & 0.0032(7) \\ \mathrm{Cl}(5) & 0.035(1) & 0.0199(9) & 0.0236(9) & -0.0041(8) & 0.0068(8) & -0.0034(7) \\ \mathrm{Cl}(1) & 0.046(1) & 0.036(1) & 0.0167(9) & 0.013(1) & 0.0046(8) & -0.0006(8) \\ \mathrm{C}(510) & 0.031(5) & 0.026(5) & 0.042(5) & -0.004(4) & -0.004(4) & 0.009(4) \\ \mathrm{C}(303) & 0.021(4) & 0.016(4) & 0.030(4) & -0.001(3) & 0.004(3) & 0.000(3) \\ \mathrm{C}(102) & 0.025(4) & 0.022(4) & 0.027(4) & 0.001(3) & 0.008(3) & -0.002(3) \\ \mathrm{C}(314) & 0.026(4) & 0.030(5) & 0.035(5) & -0.003(4) & 0.003(4) & -0.006(4) \\ \mathrm{C}(604) & 0.025(4) & 0.017(4) & 0.022(4) & 0.001(3) & -0.001(3) & 0.001(3) \\ \mathrm{C}(309) & 0.019(4) & 0.023(4) & 0.036(5) & -0.003(3) & 0.002(3) & -0.008(4) \\ \mathrm{C}(403) & 0.018(4) & 0.013(3) & 0.025(4) & 0.000(3) & 0.001(3) & 0.001(3) \\ \mathrm{C}(213) & 0.036(5) & 0.043(6) & 0.044(6) & 0.007(5) & 0.013(4) & 0.007(5) \\ \mathrm{C}(316) & 0.023(4) & 0.020(4) & 0.027(4) & 0.003(3) & 0.001(3) & -0.001(3) \\ \mathrm{C}(401) & 0.020(4) & 0.021(4) & 0.016(3) & -0.002(3) & -0.001(3) & -0.001(3) \\ \mathrm{C}(405) & 0.012(3) & 0.019(4) & 0.020(3) & 0.002(3) & 0.003(3) & 0.000(3) \\ \mathrm{C}(104) & 0.022(4) & 0.026(4) & 0.021(4) & -0.001(3) & 0.002(3) & -0.004(3) \\ \mathrm{C}(116) & 0.026(4) & 0.021(4) & 0.022(4) & -0.003(3) & 0.005(3) & -0.007(3) \\ \mathrm{C}(109) & 0.025(4) & 0.035(5) & 0.024(4) & 0.005(4) & 0.004(3) & 0.001(4)\end{array}$




\begin{tabular}{|c|c|c|c|c|c|c|}
\hline C(410) & $0.027(5)$ & $0.060(7)$ & $0.032(5)$ & $-0.010(5)$ & $0.009(4)$ & $-0.008(5)$ \\
\hline C(413) & $0.036(5)$ & $0.029(5)$ & $0.047(6)$ & $-0.002(4)$ & $0.017(4)$ & $0.005(4)$ \\
\hline C(616) & $0.028(4)$ & $0.017(4)$ & $0.029(4)$ & $0.003(3)$ & $-0.003(3)$ & $0.001(3)$ \\
\hline $\mathrm{C}(605)$ & $0.021(4)$ & $0.018(4)$ & $0.017(3)$ & $0.002(3)$ & $0.002(3)$ & $-0.000(3)$ \\
\hline C(408) & $0.021(4)$ & $0.022(4)$ & $0.033(4)$ & $0.008(3)$ & $0.001(3)$ & $0.003(3)$ \\
\hline$C(601)$ & $0.017(4)$ & $0.027(4)$ & $0.031(4)$ & $-0.002(3)$ & $0.007(3)$ & $-0.005(4)$ \\
\hline C(306) & $0.017(3)$ & $0.028(4)$ & $0.019(3)$ & $-0.003(3)$ & $0.001(3)$ & $0.005(3)$ \\
\hline C(417) & $0.035(5)$ & $0.034(5)$ & $0.021(4)$ & $0.005(4)$ & $0.006(3)$ & $-0.002(4)$ \\
\hline C(406) & $0.018(4)$ & $0.019(4)$ & $0.022(4)$ & $-0.000(3)$ & $0.001(3)$ & $-0.001(3)$ \\
\hline$C(304)$ & $0.020(4)$ & $0.025(4)$ & $0.030(4)$ & $0.002(3)$ & $0.003(3)$ & $-0.003(3)$ \\
\hline C(114) & $0.029(5)$ & $0.043(6)$ & $0.032(5)$ & $0.001(4)$ & $0.008(4)$ & $-0.010(4)$ \\
\hline$C(318)$ & $0.019(4)$ & $0.043(5)$ & $0.031(4)$ & $-0.002(4)$ & $0.002(3)$ & $-0.010(4)$ \\
\hline$C(503)$ & $0.025(4)$ & $0.014(4)$ & $0.037(5)$ & $-0.001(3)$ & $-0.002(3)$ & $-0.004(3)$ \\
\hline C(610) & $0.021(4)$ & $0.035(5)$ & $0.025(4)$ & $-0.000(4)$ & $-0.001(3)$ & $-0.003(4)$ \\
\hline C(402) & $0.018(3)$ & $0.017(4)$ & $0.021(4)$ & $0.003(3)$ & $0.002(3)$ & $0.005(3)$ \\
\hline$C(515)$ & $0.021(4)$ & $0.024(4)$ & $0.029(4)$ & $0.003(3)$ & $0.002(3)$ & $0.000(3)$ \\
\hline$C(602)$ & $0.021(4)$ & $0.019(4)$ & $0.022(4)$ & $-0.001(3)$ & $0.005(3)$ & $0.001(3)$ \\
\hline C(411) & $0.040(6)$ & $0.12(1)$ & $0.031(5)$ & $-0.011(7)$ & $0.012(5)$ & $-0.021(7)$ \\
\hline $\mathrm{C}(517)$ & $0.022(4)$ & $0.046(6)$ & $0.020(4)$ & $0.004(4)$ & $-0.004(3)$ & $0.004(4)$ \\
\hline $\mathrm{C}(217)$ & $0.072(8)$ & $0.048(7)$ & $0.046(6)$ & $0.029(6)$ & $-0.027(6)$ & $-0.027(6)$ \\
\hline C(619) & $0.037(5)$ & $0.019(4)$ & $0.034(5)$ & $0.009(4)$ & $-0.008(4)$ & $-0.000(4)$ \\
\hline C(418) & $0.024(4)$ & $0.037(5)$ & $0.025(4)$ & $0.004(4)$ & $0.006(3)$ & $0.008(4)$ \\
\hline C(416) & $0.032(4)$ & $0.021(4)$ & $0.021(4)$ & $0.001(3)$ & $0.000(3)$ & $0.000(3)$ \\
\hline C(207) & $0.033(5)$ & $0.032(5)$ & $0.056(6)$ & $0.008(4)$ & $0.010(5)$ & $0.018(5)$ \\
\hline C(613) & $0.031(5)$ & $0.030(5)$ & $0.053(6)$ & $0.002(4)$ & $0.007(4)$ & $-0.016(4)$ \\
\hline C(618) & $0.044(5)$ & $0.020(4)$ & $0.038(5)$ & $-0.000(4)$ & $-0.010(4)$ & $-0.006(4)$ \\
\hline$C(310)$ & $0.025(4)$ & $0.040(6)$ & $0.043(6)$ & $-0.008(4)$ & $-0.005(4)$ & $0.003(4)$ \\
\hline C(509) & $0.020(4)$ & $0.021(4)$ & $0.039(5)$ & $0.001(3)$ & $0.006(3)$ & $0.009(4)$ \\
\hline C(103) & $0.026(4)$ & $0.021(4)$ & $0.016(3)$ & $0.002(3)$ & $0.006(3)$ & $-0.005(3)$ \\
\hline $\mathrm{C}(311)$ & $0.021(4)$ & $0.046(6)$ & $0.059(7)$ & $-0.005(4)$ & $-0.016(4)$ & $0.003(5)$ \\
\hline C(305) & $0.018(4)$ & $0.027(4)$ & $0.020(4)$ & $0.000(3)$ & $0.006(3)$ & $-0.003(3)$ \\
\hline C(206) & $0.022(4)$ & $0.021(4)$ & $0.031(4)$ & $0.002(3)$ & $0.003(3)$ & $-0.000(3)$ \\
\hline C(101) & $0.029(4)$ & $0.018(4)$ & $0.043(5)$ & $0.006(3)$ & $0.012(4)$ & $0.006(4)$ \\
\hline$C(216)$ & $0.040(5)$ & $0.038(6)$ & $0.046(6)$ & $0.021(5)$ & $-0.011(5)$ & $-0.020(5)$ \\
\hline $\mathrm{C}(111)$ & $0.038(6)$ & $0.053(7)$ & $0.042(6)$ & $0.023(5)$ & $0.004(5)$ & $0.007(5)$ \\
\hline $\mathrm{C}(218)$ & $0.10(1)$ & $0.051(7)$ & $0.035(6)$ & $0.037(8)$ & $-0.002(7)$ & $-0.004(5)$ \\
\hline C(419) & $0.029(4)$ & $0.030(4)$ & $0.031(4)$ & $-0.003(4)$ & $0.001(3)$ & $0.011(4)$ \\
\hline C(308) & $0.027(4)$ & $0.040(5)$ & $0.038(5)$ & $-0.000(4)$ & $0.006(4)$ & $-0.013(4)$ \\
\hline$C(312)$ & $0.015(4)$ & $0.039(6)$ & $0.063(7)$ & $-0.004(4)$ & $-0.002(4)$ & $-0.015(5)$ \\
\hline C(118) & $0.024(4)$ & $0.029(5)$ & $0.033(4)$ & $0.003(3)$ & $0.009(3)$ & $-0.004(4)$ \\
\hline C(603) & $0.021(4)$ & $0.016(4)$ & $0.025(4)$ & $0.001(3)$ & $0.003(3)$ & $-0.002(3)$ \\
\hline C(507) & $0.024(4)$ & $0.026(5)$ & $0.061(6)$ & $-0.003(4)$ & $0.010(4)$ & $0.005(4)$ \\
\hline$C(415)$ & $0.014(3)$ & $0.021(4)$ & $0.023(4)$ & $0.003(3)$ & $0.002(3)$ & $0.003(3)$ \\
\hline C(620) & $0.030(4)$ & $0.021(4)$ & $0.027(4)$ & $0.005(3)$ & $-0.007(3)$ & $-0.004(3)$ \\
\hline C(606) & $0.018(4)$ & $0.023(4)$ & $0.028(4)$ & $0.003(3)$ & $0.004(3)$ & $-0.001(3)$ \\
\hline$C(505)$ & $0.017(4)$ & $0.021(4)$ & $0.033(4)$ & $0.002(3)$ & $0.002(3)$ & $-0.001(3)$ \\
\hline $\mathrm{C}(210)$ & $0.040(5)$ & $0.041(5)$ & $0.024(4)$ & $0.007(4)$ & $0.007(4)$ & $0.008(4)$ \\
\hline C(108) & $0.027(4)$ & $0.037(5)$ & $0.041(5)$ & $-0.001(4)$ & $0.007(4)$ & $-0.020(4)$ \\
\hline C(412) & $0.037(6)$ & $0.078(9)$ & $0.033(5)$ & $-0.006(6)$ & $0.019(4)$ & $0.009(5)$ \\
\hline C(611) & $0.026(4)$ & $0.041(6)$ & $0.032(4)$ & $-0.006(4)$ & $-0.006(3)$ & $-0.008(4)$ \\
\hline C(106) & $0.031(5)$ & $0.021(4)$ & $0.038(5)$ & $0.004(4)$ & $0.015(4)$ & $0.007(4)$ \\
\hline $\mathrm{C}(520)$ & $0.057(6)$ & $0.035(5)$ & $0.025(5)$ & $0.015(5)$ & $0.003(4)$ & $0.000(4)$ \\
\hline C(113) & $0.030(5)$ & $0.064(8)$ & $0.037(5)$ & $-0.004(5)$ & $0.002(4)$ & $-0.012(5)$ \\
\hline
\end{tabular}




\begin{tabular}{|c|c|c|c|c|c|c|}
\hline $\mathrm{C}(211)$ & $0.053(6)$ & $0.059(7)$ & $0.022(4)$ & $0.013(6)$ & $0.005(4)$ & $0.006(4)$ \\
\hline C(105) & $0.025(4)$ & $0.019(4)$ & $0.023(4)$ & $-0.000(3)$ & $0.005(3)$ & $0.001(3)$ \\
\hline $\mathrm{C}(511)$ & $0.036(5)$ & $0.034(5)$ & $0.054(6)$ & $-0.012(4)$ & $-0.008(5)$ & $0.018(5)$ \\
\hline $\mathrm{C}(414)$ & $0.032(5)$ & $0.024(4)$ & $0.040(5)$ & $-0.004(4)$ & $0.016(4)$ & $-0.002(4)$ \\
\hline$C(203)$ & $0.024(4)$ & $0.019(4)$ & $0.055(6)$ & $-0.001(3)$ & $0.007(4)$ & $-0.008(4)$ \\
\hline$C(315)$ & $0.016(3)$ & $0.026(4)$ & $0.023(4)$ & $0.001(3)$ & $0.003(3)$ & $-0.004(3)$ \\
\hline C(208) & $0.037(5)$ & $0.022(5)$ & $0.084(8)$ & $0.007(4)$ & $0.030(6)$ & $0.015(5)$ \\
\hline $\mathrm{C}(301)$ & $0.021(4)$ & $0.036(5)$ & $0.021(4)$ & $-0.000(4)$ & $-0.001(3)$ & $-0.004(3)$ \\
\hline$C(215)$ & $0.030(5)$ & $0.027(5)$ & $0.040(5)$ & $0.013(4)$ & $-0.004(4)$ & $-0.011(4)$ \\
\hline C(209) & $0.033(5)$ & $0.021(4)$ & $0.035(5)$ & $0.002(4)$ & $0.010(4)$ & $0.008(4)$ \\
\hline C(404) & $0.017(4)$ & $0.016(4)$ & $0.025(4)$ & $0.002(3)$ & $0.002(3)$ & $-0.001(3)$ \\
\hline $\mathrm{C}(407)$ & $0.017(4)$ & $0.031(4)$ & $0.025(4)$ & $-0.001(3)$ & $-0.004(3)$ & $0.005(3)$ \\
\hline $\mathrm{C}(302)$ & $0.018(4)$ & $0.027(4)$ & $0.022(4)$ & $-0.005(3)$ & $-0.000(3)$ & $-0.003(3)$ \\
\hline C(313) & $0.022(4)$ & $0.035(5)$ & $0.044(5)$ & $-0.008(4)$ & $0.006(4)$ & $-0.013(4)$ \\
\hline$C(516)$ & $0.022(4)$ & $0.030(4)$ & $0.029(4)$ & $0.001(3)$ & $0.001(3)$ & $-0.003(3)$ \\
\hline$C(202)$ & $0.025(4)$ & $0.017(4)$ & $0.037(5)$ & $0.003(3)$ & $0.003(4)$ & $0.006(3)$ \\
\hline$C(205)$ & $0.025(4)$ & $0.017(4)$ & $0.040(5)$ & $0.005(3)$ & $0.001(4)$ & $-0.005(3)$ \\
\hline$C(120)$ & $0.029(4)$ & $0.032(5)$ & $0.028(4)$ & $0.006(4)$ & $0.007(3)$ & $0.010(4)$ \\
\hline C(420) & $0.030(4)$ & $0.021(4)$ & $0.025(4)$ & $0.001(3)$ & $0.005(3)$ & $0.002(3)$ \\
\hline C(614) & $0.021(4)$ & $0.028(5)$ & $0.048(6)$ & $-0.004(4)$ & $0.001(4)$ & $-0.011(4)$ \\
\hline C(320) & $0.027(4)$ & $0.043(5)$ & $0.020(4)$ & $-0.011(4)$ & $0.002(3)$ & $0.004(4)$ \\
\hline C(319) & $0.031(5)$ & $0.052(6)$ & $0.028(5)$ & $-0.015(4)$ & $0.010(4)$ & $-0.001(4)$ \\
\hline C(204) & $0.030(5)$ & $0.020(4)$ & $0.066(7)$ & $-0.003(4)$ & $0.010(5)$ & $-0.013(4)$ \\
\hline C(110) & $0.039(5)$ & $0.041(6)$ & $0.040(5)$ & $0.015(5)$ & $0.004(4)$ & $0.005(5)$ \\
\hline C(107) & $0.031(5)$ & $0.019(4)$ & $0.065(7)$ & $0.001(4)$ & $0.008(5)$ & $-0.007(4)$ \\
\hline C(612) & $0.037(5)$ & $0.030(5)$ & $0.031(5)$ & $-0.007(4)$ & $-0.003(4)$ & $-0.011(4)$ \\
\hline C(617) & $0.032(5)$ & $0.023(4)$ & $0.039(5)$ & $-0.002(4)$ & $-0.008(4)$ & $-0.004(4)$ \\
\hline C(112) & $0.032(5)$ & $0.077(9)$ & $0.034(5)$ & $0.022(6)$ & $-0.001(4)$ & $0.000(5)$ \\
\hline $\mathrm{C}(519)$ & $0.066(7)$ & $0.027(5)$ & $0.039(6)$ & $0.015(5)$ & $0.009(5)$ & $-0.003(4)$ \\
\hline C(317) & $0.029(4)$ & $0.028(4)$ & $0.025(4)$ & $0.006(4)$ & $-0.008(3)$ & $-0.003(4)$ \\
\hline$C(607)$ & $0.047(5)$ & $0.025(4)$ & $0.029(4)$ & $-0.009(4)$ & $0.021(4)$ & $-0.004(4)$ \\
\hline C(115) & $0.025(4)$ & $0.016(3)$ & $0.025(4)$ & $-0.002(3)$ & $0.005(3)$ & $-0.001(3)$ \\
\hline C(409) & $0.020(4)$ & $0.024(4)$ & $0.019(3)$ & $0.005(3)$ & $0.002(3)$ & $0.005(3)$ \\
\hline$C(514)$ & $0.037(5)$ & $0.023(4)$ & $0.052(6)$ & $0.003(4)$ & $0.021(5)$ & $0.007(4)$ \\
\hline C(508) & $0.022(4)$ & $0.029(5)$ & $0.064(7)$ & $-0.002(4)$ & $-0.003(4)$ & $0.000(5)$ \\
\hline C(219) & $0.079(9)$ & $0.047(7)$ & $0.033(5)$ & $0.013(6)$ & $0.015(6)$ & $-0.004(5)$ \\
\hline$C(502)$ & $0.025(4)$ & $0.017(4)$ & $0.036(5)$ & $-0.001(3)$ & $0.006(3)$ & $0.004(3)$ \\
\hline C(307) & $0.029(5)$ & $0.040(5)$ & $0.026(4)$ & $-0.008(4)$ & $0.004(4)$ & $-0.009(4)$ \\
\hline C(615) & $0.026(4)$ & $0.020(4)$ & $0.018(3)$ & $0.005(3)$ & $0.002(3)$ & $-0.001(3)$ \\
\hline$C(201)$ & $0.025(4)$ & $0.025(4)$ & $0.031(4)$ & $0.004(3)$ & $0.001(3)$ & $0.004(4)$ \\
\hline C(119) & $0.038(5)$ & $0.036(5)$ & $0.033(5)$ & $0.004(4)$ & $0.017(4)$ & $0.005(4)$ \\
\hline C(506) & $0.020(4)$ & $0.022(4)$ & $0.032(4)$ & $0.006(3)$ & $0.008(3)$ & $0.001(3)$ \\
\hline$C(212)$ & $0.060(7)$ & $0.052(7)$ & $0.031(5)$ & $0.021(6)$ & $0.019(5)$ & $0.013(5)$ \\
\hline C(512) & $0.017(4)$ & $0.034(5)$ & $0.073(8)$ & $-0.002(4)$ & $0.001(4)$ & $0.025(5)$ \\
\hline C(214) & $0.031(5)$ & $0.025(5)$ & $0.050(6)$ & $0.001(4)$ & $0.012(4)$ & $0.003(4)$ \\
\hline C(608) & $0.041(5)$ & $0.022(4)$ & $0.024(4)$ & $0.002(4)$ & $0.007(4)$ & $0.001(3)$ \\
\hline C(518) & $0.033(5)$ & $0.033(5)$ & $0.034(5)$ & $0.010(4)$ & $-0.005(4)$ & $0.012(4)$ \\
\hline C(117) & $0.027(4)$ & $0.024(4)$ & $0.030(4)$ & $0.003(3)$ & $0.002(3)$ & $-0.004(3)$ \\
\hline C(501) & $0.025(4)$ & $0.022(4)$ & $0.038(5)$ & $0.003(3)$ & $0.011(3)$ & $0.007(4)$ \\
\hline C(504) & $0.023(4)$ & $0.024(4)$ & $0.036(5)$ & $-0.002(4)$ & $-0.004(3)$ & $0.001(4)$ \\
\hline$C(220)$ & $0.053(6)$ & $0.039(6)$ & $0.030(5)$ & $0.012(5)$ & $0.003(4)$ & $-0.006(4)$ \\
\hline C(609) & $0.022(4)$ & $0.015(4)$ & $0.021(4)$ & $-0.003(3)$ & $0.002(3)$ & $0.003(3)$ \\
\hline $\mathrm{C}(513)$ & $0.031(5)$ & $0.033(5)$ & $0.065(7)$ & $0.010(4)$ & $0.020(5)$ & $0.011(5)$ \\
\hline
\end{tabular}


The general temperature factor expression: $\exp \left(-2 \square^{2}\left(a^{\star} 2 U_{11} h^{2}+b^{\star} 2 U_{22} k^{2}+c^{\star} 2 U_{33} 1^{2}+2 a^{\star} b^{\star} U_{12} h k+\right.\right.$ $\left.2 a^{\star} c^{\star} U_{13} h l+2 b^{\star} c^{\star} U_{23} k l\right)$ )

Table S6. Bond lengths $(\AA)$

\begin{tabular}{|c|c|c|c|c|c|}
\hline atom & atom & distance & atom & atom & distance \\
\hline $\mathrm{Rh}(5)$ & $\operatorname{Rh}(6)$ & $2.904(2)$ & $\operatorname{Rh}(5)$ & $\mathrm{Cl}(6)$ & $2.405(3)$ \\
\hline $\operatorname{Rh}(5)$ & $\mathrm{Cl}(5)$ & $2.407(3)$ & $\mathrm{Rh}(5)$ & $\mathrm{C}(503)$ & $2.106(8)$ \\
\hline $\mathrm{Rh}(5)$ & $\mathrm{C}(505)$ & $2.154(8)$ & $\mathrm{Rh}(5)$ & $\mathrm{C}(502)$ & $2.135(8)$ \\
\hline $\operatorname{Rh}(5)$ & $\mathrm{C}(506)$ & $2.095(8)$ & $\mathrm{Rh}(2)$ & $\mathrm{Rh}(1)$ & $2.884(2)$ \\
\hline $\mathrm{Rh}(2)$ & $\mathrm{Cl}(2)$ & $2.403(3)$ & $\mathrm{Rh}(2)$ & $\mathrm{Cl}(1)$ & $2.401(3)$ \\
\hline $\mathrm{Rh}(2)$ & $\mathrm{C}(206)$ & $2.098(8)$ & $\mathrm{Rh}(2)$ & $\mathrm{C}(203)$ & $2.09(1)$ \\
\hline $\mathrm{Rh}(2)$ & $\mathrm{C}(202)$ & $2.140(9)$ & $\operatorname{Rh}(2)$ & $C(205)$ & $2.120(8)$ \\
\hline $\operatorname{Rh}(3)$ & $\mathrm{Rh}(4)$ & $2.981(2)$ & $\operatorname{Rh}(3)$ & $\mathrm{Cl}(3)$ & $2.398(3)$ \\
\hline $\mathrm{Rh}(3)$ & $\mathrm{Cl}(4)$ & $2.420(2)$ & $\mathrm{Rh}(3)$ & $\mathrm{C}(303)$ & $2.112(8)$ \\
\hline $\mathrm{Rh}(3)$ & $\mathrm{C}(306)$ & $2.097(7)$ & $\mathrm{Rh}(3)$ & $C(305)$ & $2.139(8)$ \\
\hline $\operatorname{Rh}(3)$ & $\mathrm{C}(302)$ & $2.153(8)$ & $\mathrm{Rh}(4)$ & $\mathrm{Cl}(3)$ & $2.397(3)$ \\
\hline $\operatorname{Rh}(4)$ & $\mathrm{Cl}(4)$ & $2.384(3)$ & $\mathrm{Rh}(4)$ & $C(403)$ & $2.099(8)$ \\
\hline $\mathrm{Rh}(4)$ & $\mathrm{C}(405)$ & $2.132(7)$ & $\operatorname{Rh}(4)$ & $\mathrm{C}(406)$ & $2.119(8)$ \\
\hline $\mathrm{Rh}(4)$ & $\mathrm{C}(402)$ & $2.138(7)$ & $\operatorname{Rh}(6)$ & $\mathrm{Cl}(6)$ & $2.402(2)$ \\
\hline $\operatorname{Rh}(6)$ & $\mathrm{Cl}(5)$ & $2.392(2)$ & $\operatorname{Rh}(6)$ & $\mathrm{C}(605)$ & $2.145(8)$ \\
\hline $\operatorname{Rh}(6)$ & $C(602)$ & $2.125(8)$ & $\operatorname{Rh}(6)$ & C(603) & $2.097(8)$ \\
\hline $\operatorname{Rh}(6)$ & $C(606)$ & $2.106(8)$ & $\operatorname{Rh}(1)$ & $\mathrm{Cl}(2)$ & $2.404(3)$ \\
\hline $\operatorname{Rh}(1)$ & $\mathrm{Cl}(1)$ & $2.385(2)$ & $\operatorname{Rh}(1)$ & $\mathrm{C}(102)$ & $2.170(8)$ \\
\hline $\operatorname{Rh}(1)$ & $\mathrm{C}(103)$ & $2.101(7)$ & $\operatorname{Rh}(1)$ & C(106) & $2.123(9)$ \\
\hline $\operatorname{Rh}(1)$ & $\mathrm{C}(105)$ & $2.165(8)$ & $\mathrm{C}(510)$ & $\mathrm{C}(509)$ & $1.40(1)$ \\
\hline $\mathrm{C}(510)$ & $\mathrm{C}(511)$ & $1.42(1)$ & $\mathrm{C}(303)$ & $\mathrm{C}(304)$ & $1.51(1)$ \\
\hline C(303) & $\mathrm{C}(302)$ & $1.41(1)$ & $\mathrm{C}(102)$ & $\mathrm{C}(109)$ & $1.48(1)$ \\
\hline$C(102)$ & $\mathrm{C}(103)$ & $1.41(1)$ & $\mathrm{C}(102)$ & $\mathrm{C}(101)$ & $1.52(1)$ \\
\hline $\mathrm{C}(314)$ & C(309) & $1.38(1)$ & $\mathrm{C}(314)$ & $\mathrm{C}(313)$ & $1.41(1)$ \\
\hline $\mathrm{C}(604)$ & $\mathrm{C}(605)$ & $1.51(1)$ & $\mathrm{C}(604)$ & $\mathrm{C}(603)$ & $1.51(1)$ \\
\hline $\mathrm{C}(604)$ & $\mathrm{C}(608)$ & $1.55(1)$ & $\mathrm{C}(309)$ & $\mathrm{C}(310)$ & $1.40(1)$ \\
\hline C(309) & $\mathrm{C}(302)$ & $1.48(1)$ & $\mathrm{C}(403)$ & $\mathrm{C}(402)$ & $1.42(1)$ \\
\hline $\mathrm{C}(403)$ & $\mathrm{C}(404)$ & $1.53(1)$ & $\mathrm{C}(213)$ & $\mathrm{C}(212)$ & $1.39(2)$ \\
\hline$C(213)$ & $\mathrm{C}(214)$ & $1.38(1)$ & $\mathrm{C}(316)$ & $\mathrm{C}(315)$ & $1.40(1)$ \\
\hline$C(316)$ & $\mathrm{C}(317)$ & $1.40(1)$ & $\mathrm{C}(401)$ & $C(406)$ & $1.50(1)$ \\
\hline$C(401)$ & $\mathrm{C}(402)$ & $1.53(1)$ & $\mathrm{C}(401)$ & $\mathrm{C}(407)$ & $1.55(1)$ \\
\hline$C(405)$ & $C(406)$ & $1.42(1)$ & $\mathrm{C}(405)$ & $\mathrm{C}(415)$ & $1.49(1)$ \\
\hline $\mathrm{C}(405)$ & $\mathrm{C}(404)$ & $1.53(1)$ & $\mathrm{C}(104)$ & $\mathrm{C}(103)$ & $1.53(1)$ \\
\hline C(104) & $\mathrm{C}(108)$ & $1.55(1)$ & $\mathrm{C}(104)$ & $\mathrm{C}(105)$ & $1.51(1)$ \\
\hline C(116) & $\mathrm{C}(115)$ & $1.38(1)$ & $\mathrm{C}(116)$ & $\mathrm{C}(117)$ & $1.40(1)$ \\
\hline C(109) & $\mathrm{C}(114)$ & $1.39(1)$ & $\mathrm{C}(109)$ & $\mathrm{C}(110)$ & $1.40(1)$ \\
\hline $\mathrm{C}(410)$ & $\mathrm{C}(411)$ & $1.41(1)$ & $\mathrm{C}(410)$ & $\mathrm{C}(409)$ & $1.36(1)$ \\
\hline $\mathrm{C}(413)$ & $\mathrm{C}(412)$ & $1.34(2)$ & $\mathrm{C}(413)$ & $\mathrm{C}(414)$ & $1.39(1)$ \\
\hline C(616) & $\mathrm{C}(617)$ & $1.39(1)$ & $\mathrm{C}(616)$ & $\mathrm{C}(615)$ & $1.40(1)$ \\
\hline$C(605)$ & $\mathrm{C}(606)$ & $1.42(1)$ & $\mathrm{C}(605)$ & $\mathrm{C}(615)$ & $1.49(1)$ \\
\hline $\mathrm{C}(408)$ & $C(404)$ & $1.53(1)$ & $\mathrm{C}(408)$ & $\mathrm{C}(407)$ & $1.54(1)$ \\
\hline $\mathrm{C}(601)$ & $\mathrm{C}(602)$ & $1.52(1)$ & $\mathrm{C}(601)$ & $C(606)$ & $1.53(1)$ \\
\hline $\mathrm{C}(601)$ & $\mathrm{C}(607)$ & $1.54(1)$ & $C(306)$ & $C(305)$ & 1.41(1) \\
\hline C(306) & $\mathrm{C}(301)$ & $1.52(1)$ & $\mathrm{C}(417)$ & $\mathrm{C}(418)$ & $1.39(1)$ \\
\hline
\end{tabular}




\begin{tabular}{|c|c|c|c|c|c|}
\hline$C(417)$ & C(416) & $1.37(1)$ & $C(304)$ & $C(305)$ & $1.54(1)$ \\
\hline$C(304)$ & C(308) & $1.57(1)$ & C(114) & C(113) & $1.40(1)$ \\
\hline$C(318)$ & $C(319)$ & $1.39(1)$ & $C(318)$ & $C(317)$ & $1.37(1)$ \\
\hline$C(503)$ & $\mathrm{C}(502)$ & $1.43(1)$ & $C(503)$ & $C(504)$ & $1.52(1)$ \\
\hline$C(610)$ & $C(611)$ & $1.38(1)$ & $C(610)$ & $C(609)$ & $1.40(1)$ \\
\hline$C(402)$ & $C(409)$ & $1.47(1)$ & $C(515)$ & $C(505)$ & $1.49(1)$ \\
\hline$C(515)$ & $C(520)$ & $1.41(1)$ & $C(515)$ & $C(516)$ & $1.41(1)$ \\
\hline$C(602)$ & $C(603)$ & $1.40(1)$ & $C(602)$ & $C(609)$ & $1.49(1)$ \\
\hline $\mathrm{C}(411)$ & $C(412)$ & $1.39(2)$ & $C(517)$ & $C(516)$ & $1.37(1)$ \\
\hline $\mathrm{C}(517)$ & $C(518)$ & $1.37(1)$ & $C(217)$ & $C(216)$ & $1.38(2)$ \\
\hline$C(217)$ & $C(218)$ & $1.36(2)$ & $C(619)$ & $C(618)$ & $1.39(1)$ \\
\hline C(619) & $C(620)$ & $1.39(1)$ & $C(418)$ & $C(419)$ & $1.37(1)$ \\
\hline$C(416)$ & $C(415)$ & $1.39(1)$ & C(207) & C(208) & $1.56(2)$ \\
\hline$C(207)$ & $\mathrm{C}(201)$ & $1.54(1)$ & C(613) & C(614) & $1.40(1)$ \\
\hline$C(613)$ & $C(612)$ & $1.38(1)$ & $C(618)$ & $C(617)$ & $1.37(1)$ \\
\hline$C(310)$ & $\mathrm{C}(311)$ & 1.41(1) & $\mathrm{C}(509)$ & $C(514)$ & $1.40(1)$ \\
\hline $\mathrm{C}(509)$ & $\mathrm{C}(502)$ & $1.48(1)$ & $\mathrm{C}(311)$ & $C(312)$ & $1.39(2)$ \\
\hline$C(305)$ & $C(315)$ & $1.47(1)$ & C(206) & $C(205)$ & $1.40(1)$ \\
\hline$C(206)$ & $\mathrm{C}(201)$ & $1.50(1)$ & $\mathrm{C}(101)$ & C(106) & $1.52(1)$ \\
\hline$C(101)$ & C(107) & $1.51(1)$ & $C(216)$ & $C(215)$ & $1.41(1)$ \\
\hline$C(111)$ & $\mathrm{C}(110)$ & $1.39(1)$ & $\mathrm{C}(111)$ & $C(112)$ & $1.36(2)$ \\
\hline$C(218)$ & $\mathrm{C}(219)$ & $1.37(2)$ & C(419) & C(420) & $1.39(1)$ \\
\hline$C(308)$ & $\mathrm{C}(307)$ & $1.55(1)$ & $C(312)$ & $\mathrm{C}(313)$ & $1.36(1)$ \\
\hline$C(118)$ & C(119) & $1.40(1)$ & C(118) & C(117) & $1.40(1)$ \\
\hline $\mathrm{C}(507)$ & $\mathrm{C}(508)$ & $1.55(2)$ & $\mathrm{C}(507)$ & $\mathrm{C}(501)$ & $1.55(1)$ \\
\hline$C(415)$ & $\mathrm{C}(420)$ & $1.39(1)$ & $\mathrm{C}(620)$ & C(615) & $1.39(1)$ \\
\hline$C(505)$ & $C(506)$ & $1.43(1)$ & $C(505)$ & $C(504)$ & $1.51(1)$ \\
\hline$C(210)$ & $\mathrm{C}(211)$ & $1.38(1)$ & $C(210)$ & $\mathrm{C}(209)$ & $1.40(1)$ \\
\hline$C(108)$ & C(107) & $1.52(1)$ & $\mathrm{C}(611)$ & $C(612)$ & $1.37(1)$ \\
\hline$C(106)$ & C(105) & $1.41(1)$ & $C(520)$ & $\mathrm{C}(519)$ & $1.38(1)$ \\
\hline$C(113)$ & $C(112)$ & $1.38(2)$ & $C(211)$ & $C(212)$ & $1.40(2)$ \\
\hline$C(105)$ & $C(115)$ & $1.48(1)$ & $\mathrm{C}(511)$ & $\mathrm{C}(512)$ & $1.39(2)$ \\
\hline$C(414)$ & C(409) & $1.40(1)$ & $C(203)$ & $C(202)$ & $1.41(1)$ \\
\hline$C(203)$ & C(204) & $1.52(1)$ & $C(315)$ & $\mathrm{C}(320)$ & $1.39(1)$ \\
\hline$C(208)$ & $C(204)$ & $1.51(2)$ & $\mathrm{C}(301)$ & $C(302)$ & $1.52(1)$ \\
\hline$C(301)$ & $\mathrm{C}(307)$ & $1.53(1)$ & $C(215)$ & $C(205)$ & $1.50(1)$ \\
\hline$C(215)$ & $\mathrm{C}(220)$ & $1.37(2)$ & C(209) & $C(202)$ & $1.47(1)$ \\
\hline C(209) & $C(214)$ & $1.42(1)$ & $C(202)$ & $\mathrm{C}(201)$ & $1.53(1)$ \\
\hline$C(205)$ & $C(204)$ & $1.55(1)$ & C(120) & $C(115)$ & $1.42(1)$ \\
\hline$C(120)$ & C(119) & $1.36(1)$ & C(614) & C(609) & $1.41(1)$ \\
\hline$C(320)$ & C(319) & $1.37(1)$ & $\mathrm{C}(519)$ & $\mathrm{C}(518)$ & $1.40(1)$ \\
\hline$C(607)$ & C(608) & $1.56(1)$ & $C(514)$ & $\mathrm{C}(513)$ & $1.38(1)$ \\
\hline$C(508)$ & $C(504)$ & $1.55(1)$ & $\mathrm{C}(219)$ & $C(220)$ & $1.38(1)$ \\
\hline$C(502)$ & $\mathrm{C}(501)$ & $1.53(1)$ & $C(506)$ & $\mathrm{C}(501)$ & $1.52(1)$ \\
\hline$C(512)$ & $C(513)$ & $1.40(2)$ & & & \\
\hline
\end{tabular}

Table S7. Bond lengths involving hydrogens ( $)$

$\begin{array}{llllll}\text { atom } & \text { atom } & \text { distance } & \text { atom } & \text { atom } & \text { distance } \\ \operatorname{Rh}(5) & \operatorname{Rh}(6) & 2.904(2) & \operatorname{Rh}(5) & \mathrm{Cl}(6) & 2.405(3) \\ \operatorname{Rh}(5) & \mathrm{Cl}(5) & 2.407(3) & \operatorname{Rh}(5) & \mathrm{C}(503) & 2.106(8) \\ \operatorname{Rh}(5) & \mathrm{C}(505) & 2.154(8) & \operatorname{Rh}(5) & \mathrm{C}(502) & 2.135(8)\end{array}$




\begin{tabular}{|c|c|c|c|c|c|}
\hline $\operatorname{Rh}(5)$ & $C(506)$ & $2.095(8)$ & $\operatorname{Rh}(2)$ & $\operatorname{Rh}(1)$ & $2.884(2)$ \\
\hline $\operatorname{Rh}(2)$ & $\mathrm{Cl}(2)$ & $2.403(3)$ & $\operatorname{Rh}(2)$ & $\mathrm{Cl}(1)$ & $2.401(3)$ \\
\hline $\operatorname{Rh}(2)$ & $C(206)$ & $2.098(8)$ & $\operatorname{Rh}(2)$ & $C(203)$ & $2.09(1)$ \\
\hline $\operatorname{Rh}(2)$ & $C(202)$ & $2.140(9)$ & $\operatorname{Rh}(2)$ & $C(205)$ & $2.120(8)$ \\
\hline $\operatorname{Rh}(3)$ & $\operatorname{Rh}(4)$ & $2.981(2)$ & $\operatorname{Rh}(3)$ & $\mathrm{Cl}(3)$ & $2.398(3)$ \\
\hline $\operatorname{Rh}(3)$ & $\mathrm{Cl}(4)$ & $2.420(2)$ & $\operatorname{Rh}(3)$ & $C(303)$ & $2.112(8)$ \\
\hline $\operatorname{Rh}(3)$ & $C(306)$ & $2.097(7)$ & $\operatorname{Rh}(3)$ & $C(305)$ & $2.139(8)$ \\
\hline $\operatorname{Rh}(3)$ & $C(302)$ & $2.153(8)$ & $\operatorname{Rh}(4)$ & $\mathrm{Cl}(3)$ & $2.397(3)$ \\
\hline $\operatorname{Rh}(4)$ & $\mathrm{Cl}(4)$ & $2.384(3)$ & $\operatorname{Rh}(4)$ & C(403) & $2.099(8)$ \\
\hline $\operatorname{Rh}(4)$ & $C(405)$ & $2.132(7)$ & $\mathrm{Rh}(4)$ & $C(406)$ & $2.119(8)$ \\
\hline $\operatorname{Rh}(4)$ & $\mathrm{C}(402)$ & $2.138(7)$ & $\operatorname{Rh}(6)$ & $\mathrm{Cl}(6)$ & $2.402(2)$ \\
\hline $\operatorname{Rh}(6)$ & $\mathrm{Cl}(5)$ & $2.392(2)$ & $\operatorname{Rh}(6)$ & C(605) & $2.145(8)$ \\
\hline $\operatorname{Rh}(6)$ & $C(602)$ & $2.125(8)$ & $\operatorname{Rh}(6)$ & C(603) & $2.097(8)$ \\
\hline $\operatorname{Rh}(6)$ & C(606) & $2.106(8)$ & $\operatorname{Rh}(1)$ & $\mathrm{Cl}(2)$ & $2.404(3)$ \\
\hline $\operatorname{Rh}(1)$ & $\mathrm{Cl}(1)$ & $2.385(2)$ & $\operatorname{Rh}(1)$ & $C(102)$ & $2.170(8)$ \\
\hline $\operatorname{Rh}(1)$ & $C(103)$ & $2.101(7)$ & $\operatorname{Rh}(1)$ & $C(106)$ & $2.123(9)$ \\
\hline $\operatorname{Rh}(1)$ & C(105) & $2.165(8)$ & $C(510)$ & $\mathrm{H}(509)$ & $0.95(1)$ \\
\hline$C(303)$ & $\mathrm{H}(302)$ & $0.95(1)$ & $C(314)$ & $\mathrm{H}(313)$ & $0.95(2)$ \\
\hline$C(604)$ & $\mathrm{H}(603)$ & $0.95(1)$ & $\mathrm{C}(403)$ & $\mathrm{H}(402)$ & $0.95(1)$ \\
\hline$C(213)$ & $\mathrm{H}(212)$ & $0.95(1)$ & $C(316)$ & $\mathrm{H}(314)$ & $0.95(1)$ \\
\hline$C(401)$ & $\mathrm{H}(401)$ & $0.95(1)$ & $\mathrm{C}(104)$ & $\mathrm{H}(103)$ & $0.95(1)$ \\
\hline$C(116)$ & $\mathrm{H}(114)$ & $0.95(1)$ & $C(410)$ & $\mathrm{H}(409)$ & $0.95(1)$ \\
\hline$C(413)$ & $\mathrm{H}(412)$ & $0.95(1)$ & $C(616)$ & $\mathrm{H}(614)$ & $0.95(1)$ \\
\hline$C(408)$ & $\mathrm{H}(408)$ & $0.95(1)$ & C(408) & $\mathrm{H}(407)$ & $0.95(1)$ \\
\hline$C(601)$ & $\mathrm{H}(601)$ & $0.95(1)$ & $C(306)$ & $\mathrm{H}(304)$ & $0.95(1)$ \\
\hline$C(417)$ & $\mathrm{H}(415)$ & $0.95(1)$ & C(406) & $\mathrm{H}(404)$ & $0.95(1)$ \\
\hline$C(304)$ & $\mathrm{H}(303)$ & $0.95(2)$ & $C(114)$ & $\mathrm{H}(113)$ & $0.95(1)$ \\
\hline$C(318)$ & $\mathrm{H}(316)$ & $0.95(1)$ & $C(503)$ & $\mathrm{H}(502)$ & $0.95(1)$ \\
\hline$C(610)$ & $\mathrm{H}(609)$ & $0.95(1)$ & $\mathrm{C}(411)$ & $\mathrm{H}(410)$ & $0.95(2)$ \\
\hline$C(517)$ & $\mathrm{H}(515)$ & $0.95(1)$ & $C(217)$ & $\mathrm{H}(215)$ & $0.95(2)$ \\
\hline$C(619)$ & $\mathrm{H}(617)$ & $0.95(1)$ & $\mathrm{C}(418)$ & $\mathrm{H}(416)$ & $0.95(1)$ \\
\hline$C(416)$ & $\mathrm{H}(414)$ & $0.95(1)$ & $C(207)$ & $\mathrm{H}(206)$ & $0.95(2)$ \\
\hline$C(207)$ & $\mathrm{H}(205)$ & $0.95(1)$ & $C(613)$ & $\mathrm{H}(612)$ & $0.95(1)$ \\
\hline C(618) & $\mathrm{H}(616)$ & $0.95(1)$ & $C(310)$ & $\mathrm{H}(309)$ & $0.95(2)$ \\
\hline C(103) & $\mathrm{H}(102)$ & $0.95(1)$ & $C(311)$ & $\mathrm{H}(310)$ & $0.95(2)$ \\
\hline$C(206)$ & $\mathrm{H}(204)$ & $0.95(1)$ & $\mathrm{C}(101)$ & $\mathrm{H}(101)$ & $0.95(1)$ \\
\hline$C(216)$ & $\mathrm{H}(214)$ & $0.95(2)$ & $C(111)$ & $\mathrm{H}(110)$ & $0.95(2)$ \\
\hline$C(218)$ & $\mathrm{H}(216)$ & $0.95(2)$ & C(419) & $\mathrm{H}(417)$ & $0.95(1)$ \\
\hline$C(308)$ & $\mathrm{H}(308)$ & $0.95(1)$ & C(308) & $\mathrm{H}(307)$ & $0.95(1)$ \\
\hline$C(312)$ & $\mathrm{H}(311)$ & $0.95(1)$ & C(118) & $\mathrm{H}(116)$ & $0.95(1)$ \\
\hline$C(603)$ & $\mathrm{H}(602)$ & $0.95(1)$ & $\mathrm{C}(507)$ & $\mathrm{H}(506)$ & $0.95(1)$ \\
\hline$C(507)$ & $\mathrm{H}(505)$ & $0.95(1)$ & $C(620)$ & $\mathrm{H}(618)$ & $0.95(1)$ \\
\hline$C(606)$ & $\mathrm{H}(604)$ & $0.95(1)$ & $C(210)$ & $\mathrm{H}(209)$ & $0.95(1)$ \\
\hline C(108) & $\mathrm{H}(108)$ & $0.95(2)$ & C(108) & $\mathrm{H}(107)$ & $0.95(1)$ \\
\hline$C(412)$ & $\mathrm{H}(411)$ & $0.95(2)$ & C(611) & $\mathrm{H}(610)$ & $0.95(1)$ \\
\hline C(106) & $\mathrm{H}(104)$ & $0.95(2)$ & $C(520)$ & $\mathrm{H}(518)$ & $0.95(1)$ \\
\hline $\mathrm{C}(113)$ & $\mathrm{H}(112)$ & $0.95(2)$ & $C(211)$ & $\mathrm{H}(210)$ & $0.95(2)$ \\
\hline $\mathrm{C}(511)$ & $\mathrm{H}(510)$ & $0.95(2)$ & C(414) & $\mathrm{H}(413)$ & $0.95(1)$ \\
\hline$C(203)$ & $\mathrm{H}(202)$ & $0.95(1)$ & $\mathrm{C}(208)$ & $\mathrm{H}(207)$ & $0.95(2)$ \\
\hline$C(208)$ & $\mathrm{H}(208)$ & $0.95(2)$ & C(301) & $\mathrm{H}(301)$ & $0.95(2)$ \\
\hline$C(404)$ & $\mathrm{H}(403)$ & $0.95(1)$ & C(407) & $\mathrm{H}(405)$ & $0.95(1)$ \\
\hline$C(407)$ & $\mathrm{H}(406)$ & $0.95(1)$ & C(313) & $\mathrm{H}(312)$ & $0.95(2)$ \\
\hline$C(516)$ & $\mathrm{H}(514)$ & $0.95(1)$ & $\mathrm{C}(120)$ & $\mathrm{H}(118)$ & $0.95(1)$ \\
\hline
\end{tabular}




$\begin{array}{llllll}\mathrm{C}(420) & \mathrm{H}(418) & 0.95(1) & \mathrm{C}(614) & \mathrm{H}(613) & 0.95(1) \\ \mathrm{C}(320) & \mathrm{H}(318) & 0.95(1) & \mathrm{C}(319) & \mathrm{H}(317) & 0.95(2) \\ \mathrm{C}(204) & \mathrm{H}(203) & 0.95(2) & \mathrm{C}(110) & \mathrm{H}(109) & 0.95(1) \\ \mathrm{C}(107) & \mathrm{H}(105) & 0.95(1) & \mathrm{C}(107) & \mathrm{H}(106) & 0.95(2) \\ \mathrm{C}(612) & \mathrm{H}(611) & 0.95(1) & \mathrm{C}(617) & \mathrm{H}(615) & 0.95(2) \\ \mathrm{C}(112) & \mathrm{H}(111) & 0.95(1) & \mathrm{C}(519) & \mathrm{H}(517) & 0.95(1) \\ \mathrm{C}(317) & \mathrm{H}(315) & 0.95(1) & \mathrm{C}(607) & \mathrm{H}(605) & 0.95(1) \\ \mathrm{C}(607) & \mathrm{H}(606) & 0.95(1) & \mathrm{C}(514) & \mathrm{H}(513) & 0.95(2) \\ \mathrm{C}(508) & \mathrm{H}(507) & 0.95(1) & \mathrm{C}(508) & \mathrm{H}(508) & 0.95(1) \\ \mathrm{C}(219) & \mathrm{H}(217) & 0.95(2) & \mathrm{C}(307) & \mathrm{H}(306) & 0.95(1) \\ \mathrm{C}(307) & \mathrm{H}(305) & 0.95(2) & \mathrm{C}(201) & \mathrm{H}(201) & 0.95(1) \\ \mathrm{C}(119) & \mathrm{H}(117) & 0.95(2) & \mathrm{C}(506) & \mathrm{H}(504) & 0.95(1) \\ \mathrm{C}(212) & \mathrm{H}(211) & 0.95(2) & \mathrm{C}(512) & \mathrm{H}(511) & 0.95(2) \\ \mathrm{C}(214) & \mathrm{H}(213) & 0.95(2) & \mathrm{C}(608) & \mathrm{H}(608) & 0.95(1) \\ \mathrm{C}(608) & \mathrm{H}(607) & 0.95(1) & \mathrm{C}(518) & \mathrm{H}(516) & 0.95(1) \\ \mathrm{C}(117) & \mathrm{H}(115) & 0.95(1) & \mathrm{C}(501) & \mathrm{H}(501) & 0.95(1) \\ \mathrm{C}(504) & \mathrm{H}(503) & 0.95(1) & \mathrm{C}(220) & \mathrm{H}(218) & 0.95(2) \\ \mathrm{C}(513) & \mathrm{H}(512) & 0.95(2) & & & \end{array}$

Table S8. Bond angles (')

\begin{tabular}{|c|c|c|c|c|c|c|c|}
\hline atom & atom & atom & angle & atom & atom & atom & angle \\
\hline $\mathrm{Rh}(6)$ & $\mathrm{Rh}(5)$ & $\mathrm{Cl}(6)$ & $52.79(7)$ & $\operatorname{Rh}(6)$ & $\mathrm{Rh}(5)$ & $\mathrm{Cl}(5)$ & $52.53(6)$ \\
\hline $\mathrm{Cl}(6)$ & $\operatorname{Rh}(5)$ & $\mathrm{Cl}(5)$ & $84.62(7)$ & $\operatorname{Rh}(6)$ & $\operatorname{Rh}(5)$ & $\mathrm{C}(503)$ & $117.8(3)$ \\
\hline $\mathrm{Cl}(6)$ & $\operatorname{Rh}(5)$ & $\mathrm{C}(503)$ & $99.5(2)$ & $\mathrm{Cl}(5)$ & $\operatorname{Rh}(5)$ & $\mathrm{C}(503)$ & $163.3(2)$ \\
\hline $\operatorname{Rh}(6)$ & $\operatorname{Rh}(5)$ & $\mathrm{C}(505)$ & $113.4(2)$ & $\mathrm{Cl}(6)$ & $\operatorname{Rh}(5)$ & $\mathrm{C}(505)$ & $154.3(2)$ \\
\hline $\mathrm{Cl}(5)$ & $\operatorname{Rh}(5)$ & $C(505)$ & $103.6(2)$ & $C(503)$ & $\operatorname{Rh}(5)$ & $\mathrm{C}(505)$ & $66.1(3)$ \\
\hline $\operatorname{Rh}(6)$ & $\operatorname{Rh}(5)$ & $C(502)$ & $147.8(2)$ & $\mathrm{Cl}(6)$ & $\operatorname{Rh}(5)$ & $\mathrm{C}(502)$ & $101.9(2)$ \\
\hline $\mathrm{Cl}(5)$ & $\operatorname{Rh}(5)$ & $\mathrm{C}(502)$ & $155.7(3)$ & $C(503)$ & $\operatorname{Rh}(5)$ & $\mathrm{C}(502)$ & $39.3(3)$ \\
\hline$C(505)$ & $\operatorname{Rh}(5)$ & $\mathrm{C}(502)$ & $80.7(3)$ & $\operatorname{Rh}(6)$ & $\operatorname{Rh}(5)$ & $\mathrm{C}(506)$ & $141.5(2)$ \\
\hline $\mathrm{Cl}(6)$ & $\operatorname{Rh}(5)$ & $C(506)$ & $163.9(2)$ & $\mathrm{Cl}(5)$ & $\operatorname{Rh}(5)$ & $C(506)$ & $100.6(3)$ \\
\hline$C(503)$ & $\operatorname{Rh}(5)$ & $C(506)$ & $79.8(3)$ & $C(505)$ & $\operatorname{Rh}(5)$ & $C(506)$ & $39.3(3)$ \\
\hline $\mathrm{C}(502)$ & $\operatorname{Rh}(5)$ & $C(506)$ & $67.3(3)$ & $\operatorname{Rh}(1)$ & $\operatorname{Rh}(2)$ & $\mathrm{Cl}(2)$ & $53.16(6)$ \\
\hline $\operatorname{Rh}(1)$ & $\operatorname{Rh}(2)$ & $\mathrm{Cl}(1)$ & $52.70(6)$ & $\mathrm{Cl}(2)$ & $\operatorname{Rh}(2)$ & $\mathrm{Cl}(1)$ & $82.7(1)$ \\
\hline $\mathrm{Rh}(1)$ & $\operatorname{Rh}(2)$ & $C(206)$ & $113.8(2)$ & $\mathrm{Cl}(2)$ & $\operatorname{Rh}(2)$ & $C(206)$ & $162.5(2)$ \\
\hline $\mathrm{Cl}(1)$ & $\operatorname{Rh}(2)$ & $C(206)$ & $98.0(3)$ & $\operatorname{Rh}(1)$ & $\operatorname{Rh}(2)$ & $\mathrm{C}(203)$ & $139.9(3)$ \\
\hline $\mathrm{Cl}(2)$ & $\operatorname{Rh}(2)$ & $\mathrm{C}(203)$ & $103.3(3)$ & $\mathrm{Cl}(1)$ & $\operatorname{Rh}(2)$ & $\mathrm{C}(203)$ & $167.0(3)$ \\
\hline C(206) & $\operatorname{Rh}(2)$ & $\mathrm{C}(203)$ & $79.8(4)$ & $\operatorname{Rh}(1)$ & $\operatorname{Rh}(2)$ & $C(202)$ & $109.3(2)$ \\
\hline $\mathrm{Cl}(2)$ & $\operatorname{Rh}(2)$ & $\mathrm{C}(202)$ & $104.4(2)$ & $\mathrm{Cl}(1)$ & $\operatorname{Rh}(2)$ & $C(202)$ & $151.3(3)$ \\
\hline C(206) & $\operatorname{Rh}(2)$ & $\mathrm{C}(202)$ & $67.0(3)$ & $C(203)$ & $\operatorname{Rh}(2)$ & $C(202)$ & $38.8(4)$ \\
\hline $\mathrm{Rh}(1)$ & $\operatorname{Rh}(2)$ & $C(205)$ & $145.5(2)$ & $\mathrm{Cl}(2)$ & $\operatorname{Rh}(2)$ & $C(205)$ & $158.1(3)$ \\
\hline $\mathrm{Cl}(1)$ & $\operatorname{Rh}(2)$ & $C(205)$ & $102.6(3)$ & $C(206)$ & $\mathrm{Rh}(2)$ & $C(205)$ & $38.8(3)$ \\
\hline C(203) & $\operatorname{Rh}(2)$ & $C(205)$ & $67.6(4)$ & $C(202)$ & $\operatorname{Rh}(2)$ & $C(205)$ & $81.3(4)$ \\
\hline $\mathrm{Rh}(4)$ & $\operatorname{Rh}(3)$ & $\mathrm{Cl}(3)$ & $51.55(7)$ & $\mathrm{Rh}(4)$ & $\operatorname{Rh}(3)$ & $\mathrm{Cl}(4)$ & $51.10(6)$ \\
\hline $\mathrm{Cl}(3)$ & $\operatorname{Rh}(3)$ & $\mathrm{Cl}(4)$ & $84.60(9)$ & $\operatorname{Rh}(4)$ & $\operatorname{Rh}(3)$ & $C(303)$ & $138.6(2)$ \\
\hline $\mathrm{Cl}(3)$ & $\operatorname{Rh}(3)$ & $C(303)$ & $169.0(2)$ & $\mathrm{Cl}(4)$ & $\operatorname{Rh}(3)$ & $C(303)$ & $100.2(2)$ \\
\hline $\operatorname{Rh}(4)$ & $\operatorname{Rh}(3)$ & $C(306)$ & $120.5(2)$ & $\mathrm{Cl}(3)$ & $\operatorname{Rh}(3)$ & $\mathrm{C}(306)$ & $97.8(3)$ \\
\hline $\mathrm{Cl}(4)$ & $\operatorname{Rh}(3)$ & $C(306)$ & $165.9(2)$ & $C(303)$ & $\operatorname{Rh}(3)$ & C(306) & $79.9(3)$ \\
\hline $\operatorname{Rh}(4)$ & $\operatorname{Rh}(3)$ & $C(305)$ & $150.8(2)$ & $\mathrm{Cl}(3)$ & $\operatorname{Rh}(3)$ & $C(305)$ & $103.8(2)$ \\
\hline $\mathrm{Cl}(4)$ & $\operatorname{Rh}(3)$ & $C(305)$ & $153.7(2)$ & $C(303)$ & $\operatorname{Rh}(3)$ & $C(305)$ & $67.6(3)$ \\
\hline$C(306)$ & $\operatorname{Rh}(3)$ & $C(305)$ & $39.0(3)$ & $\operatorname{Rh}(4)$ & $\operatorname{Rh}(3)$ & $C(302)$ & $112.3(2)$ \\
\hline
\end{tabular}




\begin{tabular}{|c|c|c|c|c|c|c|c|}
\hline $\mathrm{Cl}(3)$ & $\operatorname{Rh}(3)$ & $C(302)$ & $149.6(2)$ & $\mathrm{Cl}(4)$ & $\operatorname{Rh}(3)$ & $C(302)$ & $104.6(2)$ \\
\hline C(303) & $\operatorname{Rh}(3)$ & $C(302)$ & $38.7(3)$ & $C(306)$ & $\operatorname{Rh}(3)$ & $C(302)$ & $66.6(3)$ \\
\hline$C(305)$ & $\operatorname{Rh}(3)$ & $C(302)$ & $80.8(3)$ & $\operatorname{Rh}(3)$ & $\operatorname{Rh}(4)$ & $\mathrm{Cl}(3)$ & $51.58(7)$ \\
\hline $\operatorname{Rh}(3)$ & $\mathrm{Rh}(4)$ & $\mathrm{Cl}(4)$ & $52.18(6)$ & $\mathrm{Cl}(3)$ & $\mathrm{Rh}(4)$ & $\mathrm{Cl}(4)$ & $85.41(8)$ \\
\hline $\operatorname{Rh}(3)$ & $\operatorname{Rh}(4)$ & $C(403)$ & $143.6(2)$ & $\mathrm{Cl}(3)$ & $\operatorname{Rh}(4)$ & $C(403)$ & $101.5(2)$ \\
\hline $\mathrm{Cl}(4)$ & $\operatorname{Rh}(4)$ & $C(403)$ & $162.3(2)$ & $\operatorname{Rh}(3)$ & $\operatorname{Rh}(4)$ & $C(405)$ & $145.2(2)$ \\
\hline $\mathrm{Cl}(3)$ & $\operatorname{Rh}(4)$ & $C(405)$ & $159.9(2)$ & $\mathrm{Cl}(4)$ & $\mathrm{Rh}(4)$ & $C(405)$ & $100.4(2)$ \\
\hline$C(403)$ & $\operatorname{Rh}(4)$ & $C(405)$ & $67.8(3)$ & $\operatorname{Rh}(3)$ & $\operatorname{Rh}(4)$ & C(406) & $116.7(2)$ \\
\hline $\mathrm{Cl}(3)$ & $\operatorname{Rh}(4)$ & C(406) & $159.4(2)$ & $\mathrm{Cl}(4)$ & $\mathrm{Rh}(4)$ & C(406) & $99.6(2)$ \\
\hline C(403) & $\operatorname{Rh}(4)$ & C(406) & 79.7(3) & $C(405)$ & $\operatorname{Rh}(4)$ & C(406) & $38.9(3)$ \\
\hline $\operatorname{Rh}(3)$ & $\operatorname{Rh}(4)$ & $C(402)$ & $114.7(2)$ & $\mathrm{Cl}(3)$ & $\operatorname{Rh}(4)$ & $C(402)$ & $101.2(2)$ \\
\hline $\mathrm{Cl}(4)$ & $\operatorname{Rh}(4)$ & $\mathrm{C}(402)$ & $155.9(2)$ & $C(403)$ & $\operatorname{Rh}(4)$ & $\mathrm{C}(402)$ & 39.1(3) \\
\hline$C(405)$ & $\operatorname{Rh}(4)$ & $\mathrm{C}(402)$ & $81.3(3)$ & $C(406)$ & $\mathrm{Rh}(4)$ & C(402) & $66.6(3)$ \\
\hline $\operatorname{Rh}(5)$ & $\operatorname{Rh}(6)$ & $\mathrm{Cl}(6)$ & $52.88(6)$ & $\operatorname{Rh}(5)$ & $\operatorname{Rh}(6)$ & $\mathrm{Cl}(5)$ & $52.99(6)$ \\
\hline $\mathrm{Cl}(6)$ & $\operatorname{Rh}(6)$ & $\mathrm{Cl}(5)$ & $84.99(9)$ & $\operatorname{Rh}(5)$ & $\operatorname{Rh}(6)$ & $C(605)$ & $111.6(2)$ \\
\hline $\mathrm{Cl}(6)$ & $\operatorname{Rh}(6)$ & $C(605)$ & $102.1(2)$ & $\mathrm{Cl}(5)$ & $\operatorname{Rh}(6)$ & $C(605)$ & $153.6(2)$ \\
\hline $\operatorname{Rh}(5)$ & $\operatorname{Rh}(6)$ & $\mathrm{C}(602)$ & $146.6(2)$ & $\mathrm{Cl}(6)$ & $\operatorname{Rh}(6)$ & $C(602)$ & $157.8(2)$ \\
\hline $\mathrm{Cl}(5)$ & $\operatorname{Rh}(6)$ & $C(602)$ & $102.2(2)$ & $C(605)$ & $\operatorname{Rh}(6)$ & $C(602)$ & $80.8(3)$ \\
\hline $\operatorname{Rh}(5)$ & $\operatorname{Rh}(6)$ & $C(603)$ & $115.9(2)$ & $\mathrm{Cl}(6)$ & $\operatorname{Rh}(6)$ & $C(603)$ & $161.5(2)$ \\
\hline $\mathrm{Cl}(5)$ & $\operatorname{Rh}(6)$ & $C(603)$ & $99.1(2)$ & $C(605)$ & $\operatorname{Rh}(6)$ & $C(603)$ & $66.7(3)$ \\
\hline$C(602)$ & $\operatorname{Rh}(6)$ & $C(603)$ & $38.8(3)$ & $\operatorname{Rh}(5)$ & $\operatorname{Rh}(6)$ & C(606) & $140.8(2)$ \\
\hline $\mathrm{Cl}(6)$ & $\operatorname{Rh}(6)$ & $\mathrm{C}(606)$ & $100.8(2)$ & $\mathrm{Cl}(5)$ & $\operatorname{Rh}(6)$ & $\mathrm{C}(606)$ & $165.0(2)$ \\
\hline$C(605)$ & $\operatorname{Rh}(6)$ & $C(606)$ & $38.9(3)$ & $C(602)$ & $\operatorname{Rh}(6)$ & $C(606)$ & $67.5(3)$ \\
\hline$C(603)$ & $\operatorname{Rh}(6)$ & $C(606)$ & $79.9(3)$ & $\mathrm{Rh}(2)$ & $\mathrm{Rh}(1)$ & $\mathrm{Cl}(2)$ & $53.13(6)$ \\
\hline $\operatorname{Rh}(2)$ & $\operatorname{Rh}(1)$ & $\mathrm{Cl}(1)$ & $53.20(6)$ & $\mathrm{Cl}(2)$ & $\operatorname{Rh}(1)$ & $\mathrm{Cl}(1)$ & $83.0(1)$ \\
\hline $\operatorname{Rh}(2)$ & $\mathrm{Rh}(1)$ & $\mathrm{C}(102)$ & $146.3(2)$ & $\mathrm{Cl}(2)$ & $\operatorname{Rh}(1)$ & $\mathrm{C}(102)$ & $106.3(2)$ \\
\hline $\mathrm{Cl}(1)$ & $\mathrm{Rh}(1)$ & $\mathrm{C}(102)$ & $159.8(2)$ & $\operatorname{Rh}(2)$ & $\mathrm{Rh}(1)$ & C(103) & $112.3(2)$ \\
\hline $\mathrm{Cl}(2)$ & $\operatorname{Rh}(1)$ & $C(103)$ & $99.2(2)$ & $\mathrm{Cl}(1)$ & $\operatorname{Rh}(1)$ & $C(103)$ & $159.6(2)$ \\
\hline$C(102)$ & $\operatorname{Rh}(1)$ & $\mathrm{C}(103)$ & 38.4(3) & $\operatorname{Rh}(2)$ & $\operatorname{Rh}(1)$ & C(106) & $136.8(2)$ \\
\hline $\mathrm{Cl}(2)$ & $\operatorname{Rh}(1)$ & C(106) & $169.9(3)$ & $\mathrm{Cl}(1)$ & $\operatorname{Rh}(1)$ & C(106) & $102.3(3)$ \\
\hline$C(102)$ & $\operatorname{Rh}(1)$ & C(106) & $65.9(3)$ & $C(103)$ & $\operatorname{Rh}(1)$ & C(106) & $78.9(3)$ \\
\hline $\operatorname{Rh}(2)$ & $\operatorname{Rh}(1)$ & C(105) & $105.5(2)$ & $\mathrm{Cl}(2)$ & $\mathrm{Rh}(1)$ & $C(105)$ & $149.7(2)$ \\
\hline $\mathrm{Cl}(1)$ & $\operatorname{Rh}(1)$ & $C(105)$ & 101.1(2) & $C(102)$ & $\operatorname{Rh}(1)$ & C(105) & $80.0(3)$ \\
\hline C(103) & $\operatorname{Rh}(1)$ & $C(105)$ & $67.0(3)$ & $C(106)$ & $\operatorname{Rh}(1)$ & $C(105)$ & 38.3(3) \\
\hline $\mathrm{Rh}(2)$ & $\mathrm{Cl}(2)$ & $\operatorname{Rh}(1)$ & $73.72(8)$ & $\operatorname{Rh}(3)$ & $\mathrm{Cl}(3)$ & $\mathrm{Rh}(4)$ & $76.86(8)$ \\
\hline $\mathrm{Rh}(3)$ & $\mathrm{Cl}(4)$ & $\operatorname{Rh}(4)$ & $76.72(7)$ & $\operatorname{Rh}(5)$ & $\mathrm{Cl}(6)$ & $\operatorname{Rh}(6)$ & $74.33(7)$ \\
\hline $\operatorname{Rh}(5)$ & $\mathrm{Cl}(5)$ & $\operatorname{Rh}(6)$ & $74.48(7)$ & $\operatorname{Rh}(2)$ & $\mathrm{Cl}(1)$ & $\operatorname{Rh}(1)$ & $74.10(8)$ \\
\hline C(509) & $C(510)$ & $\mathrm{C}(511)$ & $120.2(1)$ & $\operatorname{Rh}(3)$ & $C(303)$ & $C(304)$ & $95.0(5)$ \\
\hline $\operatorname{Rh}(3)$ & $C(303)$ & $C(302)$ & $72.2(5)$ & $C(304)$ & $C(303)$ & $C(302)$ & $114.5(7)$ \\
\hline $\mathrm{Rh}(1)$ & $C(102)$ & C(109) & $122.3(6)$ & $\mathrm{Rh}(1)$ & $C(102)$ & $\mathrm{C}(103)$ & $68.2(4)$ \\
\hline C(109) & $C(102)$ & C(103) & $124.4(8)$ & $\mathrm{Rh}(1)$ & $C(102)$ & $\mathrm{C}(101)$ & $95.0(5)$ \\
\hline C(109) & $C(102)$ & $\mathrm{C}(101)$ & $120.8(8)$ & $C(103)$ & $C(102)$ & $C(101)$ & $110.9(7)$ \\
\hline C(309) & $C(314)$ & $C(313)$ & $121.0(9)$ & $C(605)$ & $C(604)$ & $C(603)$ & $100.7(6)$ \\
\hline C(605) & $C(604)$ & $C(608)$ & $111.2(7)$ & $C(603)$ & $C(604)$ & $C(608)$ & $110.3(7)$ \\
\hline C(314) & $\mathrm{C}(309)$ & $C(310)$ & $118.0(8)$ & $C(314)$ & $C(309)$ & $C(302)$ & $121.2(8)$ \\
\hline$C(310)$ & $C(309)$ & $C(302)$ & $120.8(8)$ & $\mathrm{Rh}(4)$ & $C(403)$ & $C(402)$ & $72.0(4)$ \\
\hline $\mathrm{Rh}(4)$ & $C(403)$ & $C(404)$ & $95.4(5)$ & $\mathrm{C}(402)$ & $C(403)$ & $C(404)$ & $113.8(7)$ \\
\hline$C(212)$ & $C(213)$ & $C(214)$ & 119.7(1) & $C(315)$ & $C(316)$ & $C(317)$ & $120.1(8)$ \\
\hline C(406) & $\mathrm{C}(401)$ & $C(402)$ & $100.8(6)$ & $C(406)$ & $\mathrm{C}(401)$ & $C(407)$ & $110.9(6)$ \\
\hline C(402) & $\mathrm{C}(401)$ & $\mathrm{C}(407)$ & 109.3(6) & $\mathrm{Rh}(4)$ & $C(405)$ & C(406) & $70.0(4)$ \\
\hline $\mathrm{Rh}(4)$ & C(405) & $C(415)$ & $117.5(5)$ & $C(406)$ & $C(405)$ & $C(415)$ & $125.0(7)$ \\
\hline $\mathrm{Rh}(4)$ & $C(405)$ & C(404) & $94.0(5)$ & $C(406)$ & $C(405)$ & C(404) & $110.7(7)$ \\
\hline C(415) & $C(405)$ & C(404) & $122.1(7)$ & $C(103)$ & $C(104)$ & C(108) & $109.8(7)$ \\
\hline
\end{tabular}




\begin{tabular}{|c|c|c|c|c|c|c|c|}
\hline$C(103)$ & $C(104)$ & $C(105)$ & $101.6(6)$ & $C(108)$ & $C(104)$ & $C(105)$ & $109.7(7)$ \\
\hline$C(115)$ & $C(116)$ & C(117) & $121.3(8)$ & C(102) & C(109) & $C(114)$ & $122.5(9)$ \\
\hline$C(102)$ & $\mathrm{C}(109)$ & $\mathrm{C}(110)$ & 119.3(9) & $\mathrm{C}(114)$ & $\mathrm{C}(109)$ & $C(110)$ & 118.2(9) \\
\hline$C(411)$ & $\mathrm{C}(410)$ & C(409) & $119.8(1)$ & $\mathrm{C}(412)$ & $C(413)$ & $C(414)$ & $120.3(1)$ \\
\hline$C(617)$ & $C(616)$ & $C(615)$ & $120.6(8)$ & $\operatorname{Rh}(6)$ & $C(605)$ & $C(604)$ & $94.6(5)$ \\
\hline $\operatorname{Rh}(6)$ & $C(605)$ & $C(606)$ & $69.1(4)$ & $\mathrm{C}(604)$ & $C(605)$ & $C(606)$ & $111.3(7)$ \\
\hline $\operatorname{Rh}(6)$ & $C(605)$ & $C(615)$ & $115.4(5)$ & $C(604)$ & $\mathrm{C}(605)$ & $C(615)$ & $122.1(7)$ \\
\hline C(606) & $\mathrm{C}(605)$ & $\mathrm{C}(615)$ & $125.1(7)$ & C(404) & $\mathrm{C}(408)$ & $\mathrm{C}(407)$ & $109.2(6)$ \\
\hline$C(602)$ & $\mathrm{C}(601)$ & $C(606)$ & $101.0(6)$ & $\mathrm{C}(602)$ & $C(601)$ & $\mathrm{C}(607)$ & $111.3(7)$ \\
\hline$C(606)$ & $\mathrm{C}(601)$ & $C(607)$ & 109.1(7) & $\mathrm{Rh}(3)$ & $C(306)$ & $C(305)$ & $72.1(4)$ \\
\hline $\operatorname{Rh}(3)$ & $C(306)$ & $\mathrm{C}(301)$ & $97.2(5)$ & $C(305)$ & $C(306)$ & $\mathrm{C}(301)$ & $113.2(8)$ \\
\hline$C(418)$ & $\mathrm{C}(417)$ & C(416) & $121.4(8)$ & $\operatorname{Rh}(4)$ & $C(406)$ & $C(401)$ & $96.4(5)$ \\
\hline $\mathrm{Rh}(4)$ & C(406) & $C(405)$ & $71.0(4)$ & $C(401)$ & $C(406)$ & $C(405)$ & $114.7(7)$ \\
\hline$C(303)$ & $C(304)$ & $C(305)$ & $101.7(6)$ & C(303) & $C(304)$ & $\mathrm{C}(308)$ & $110.0(7)$ \\
\hline$C(305)$ & $C(304)$ & C(308) & 109.7(7) & C(109) & $C(114)$ & $C(113)$ & 119.9(1) \\
\hline$C(319)$ & $\mathrm{C}(318)$ & $C(317)$ & $118.8(8)$ & $\operatorname{Rh}(5)$ & $C(503)$ & $C(502)$ & $71.4(5)$ \\
\hline $\operatorname{Rh}(5)$ & $C(503)$ & $C(504)$ & $97.1(5)$ & $C(502)$ & $C(503)$ & $C(504)$ & $113.7(8)$ \\
\hline$C(611)$ & $C(610)$ & $\mathrm{C}(609)$ & $120.6(8)$ & $\operatorname{Rh}(4)$ & $C(402)$ & $C(403)$ & $69.0(4)$ \\
\hline $\mathrm{Rh}(4)$ & $\mathrm{C}(402)$ & $\mathrm{C}(401)$ & $94.8(5)$ & C(403) & $C(402)$ & $C(401)$ & $111.2(6)$ \\
\hline $\operatorname{Rh}(4)$ & $\mathrm{C}(402)$ & C(409) & $115.5(5)$ & C(403) & $C(402)$ & $\mathrm{C}(409)$ & $124.2(7)$ \\
\hline $\mathrm{C}(401)$ & $\mathrm{C}(402)$ & C(409) & $123.1(7)$ & $C(505)$ & $C(515)$ & $C(520)$ & $121.6(8)$ \\
\hline$C(505)$ & $C(515)$ & $C(516)$ & $122.0(8)$ & $C(520)$ & $C(515)$ & $C(516)$ & $116.4(8)$ \\
\hline $\operatorname{Rh}(6)$ & $C(602)$ & $\mathrm{C}(601)$ & $94.3(5)$ & $\operatorname{Rh}(6)$ & $C(602)$ & $C(603)$ & $69.5(5)$ \\
\hline$C(601)$ & $C(602)$ & $\mathrm{C}(603)$ & $112.0(7)$ & $\operatorname{Rh}(6)$ & $C(602)$ & $C(609)$ & $115.4(5)$ \\
\hline$C(601)$ & $C(602)$ & $\mathrm{C}(609)$ & $122.8(7)$ & $\mathrm{C}(603)$ & $C(602)$ & $C(609)$ & $123.7(7)$ \\
\hline$C(410)$ & $\mathrm{C}(411)$ & $C(412)$ & $120.3(1)$ & $C(516)$ & $\mathrm{C}(517)$ & $C(518)$ & $123.2(8)$ \\
\hline$C(216)$ & $C(217)$ & $\mathrm{C}(218)$ & $120.5(1)$ & C(618) & $C(619)$ & $C(620)$ & $120.5(8)$ \\
\hline$C(417)$ & $\mathrm{C}(418)$ & C(419) & $118.0(8)$ & C(417) & $C(416)$ & $C(415)$ & $121.3(8)$ \\
\hline$C(208)$ & $\mathrm{C}(207)$ & $\mathrm{C}(201)$ & 109.3(8) & $\mathrm{C}(614)$ & $C(613)$ & $C(612)$ & 119.7(9) \\
\hline$C(619)$ & $C(618)$ & $C(617)$ & 118.4(9) & $\mathrm{C}(309)$ & $C(310)$ & $C(311)$ & $120.9(9)$ \\
\hline$C(510)$ & $\mathrm{C}(509)$ & $\mathrm{C}(514)$ & 118.8(9) & $\mathrm{C}(510)$ & $\mathrm{C}(509)$ & $C(502)$ & $120.3(8)$ \\
\hline$C(514)$ & $\mathrm{C}(509)$ & $C(502)$ & $120.8(9)$ & $\operatorname{Rh}(1)$ & $C(103)$ & $C(102)$ & 73.4(5) \\
\hline $\mathrm{Rh}(1)$ & $C(103)$ & C(104) & $95.6(5)$ & $\mathrm{C}(102)$ & $C(103)$ & $C(104)$ & $113.9(7)$ \\
\hline$C(310)$ & $C(311)$ & $C(312)$ & 119.5(9) & $\operatorname{Rh}(3)$ & $C(305)$ & $C(306)$ & $68.9(4)$ \\
\hline $\operatorname{Rh}(3)$ & $C(305)$ & $C(304)$ & $93.2(5)$ & $C(306)$ & $C(305)$ & $C(304)$ & $111.8(7)$ \\
\hline $\operatorname{Rh}(3)$ & $C(305)$ & $C(315)$ & $120.0(5)$ & $C(306)$ & $C(305)$ & $C(315)$ & $124.6(8)$ \\
\hline$C(304)$ & $C(305)$ & $C(315)$ & $121.2(7)$ & $\operatorname{Rh}(2)$ & $C(206)$ & $C(205)$ & $71.4(5)$ \\
\hline $\operatorname{Rh}(2)$ & C(206) & $\mathrm{C}(201)$ & $96.5(5)$ & $\mathrm{C}(205)$ & $C(206)$ & $C(201)$ & $115.3(8)$ \\
\hline$C(102)$ & $C(101)$ & $C(106)$ & $100.6(7)$ & $\mathrm{C}(102)$ & $C(101)$ & $C(107)$ & $111.2(8)$ \\
\hline$C(106)$ & $C(101)$ & $C(107)$ & $110.3(8)$ & $\mathrm{C}(217)$ & $C(216)$ & $C(215)$ & 119.3(1) \\
\hline$C(110)$ & $\mathrm{C}(111)$ & $C(112)$ & $122.3(1)$ & $C(217)$ & $\mathrm{C}(218)$ & $C(219)$ & $120.8(1)$ \\
\hline$C(418)$ & C(419) & $C(420)$ & $120.9(9)$ & C(304) & $\mathrm{C}(308)$ & $C(307)$ & $107.6(7)$ \\
\hline$C(311)$ & $C(312)$ & $C(313)$ & 119.9(9) & C(119) & $C(118)$ & $C(117)$ & 119.7(8) \\
\hline $\operatorname{Rh}(6)$ & $C(603)$ & $C(604)$ & $96.6(5)$ & $\operatorname{Rh}(6)$ & $C(603)$ & $C(602)$ & $71.7(5)$ \\
\hline$C(604)$ & $C(603)$ & $C(602)$ & $113.7(7)$ & $C(508)$ & $\mathrm{C}(507)$ & $C(501)$ & $109.0(7)$ \\
\hline$C(405)$ & $C(415)$ & $C(416)$ & $121.3(7)$ & $C(405)$ & $C(415)$ & $C(420)$ & $121.4(7)$ \\
\hline$C(416)$ & $C(415)$ & $C(420)$ & 117.3(7) & C(619) & $C(620)$ & $C(615)$ & $121.3(8)$ \\
\hline $\operatorname{Rh}(6)$ & $C(606)$ & $C(605)$ & $72.0(4)$ & $\operatorname{Rh}(6)$ & $C(606)$ & $C(601)$ & $95.0(5)$ \\
\hline$C(605)$ & $C(606)$ & $C(601)$ & $113.8(7)$ & $\operatorname{Rh}(5)$ & $C(505)$ & $C(515)$ & 115.1(6) \\
\hline $\operatorname{Rh}(5)$ & $C(505)$ & $C(506)$ & $68.2(5)$ & $C(515)$ & $C(505)$ & $C(506)$ & $124.9(7)$ \\
\hline $\operatorname{Rh}(5)$ & $C(505)$ & $C(504)$ & $95.3(5)$ & $C(515)$ & $C(505)$ & $C(504)$ & $122.1(7)$ \\
\hline$C(506)$ & $C(505)$ & $C(504)$ & $111.7(7)$ & $\mathrm{C}(211)$ & $C(210)$ & $C(209)$ & 121.1(1) \\
\hline$C(104)$ & $\mathrm{C}(108)$ & C(107) & $109.1(8)$ & C(413) & $C(412)$ & $C(411)$ & 119.9(9) \\
\hline
\end{tabular}




\begin{tabular}{|c|c|c|c|c|c|c|c|}
\hline$C(610)$ & $C(611)$ & $C(612)$ & $121.6(9)$ & $\operatorname{Rh}(1)$ & $C(106)$ & $C(101)$ & $97.1(6)$ \\
\hline $\operatorname{Rh}(1)$ & C(106) & C(105) & $72.5(5)$ & $\mathrm{C}(101)$ & C(106) & C(105) & $113.8(8)$ \\
\hline$C(515)$ & $\mathrm{C}(520)$ & $C(519)$ & $121.6(9)$ & $\mathrm{C}(114)$ & $C(113)$ & $C(112)$ & $121.2(1)$ \\
\hline$C(210)$ & $\mathrm{C}(211)$ & $C(212)$ & $120.6(1)$ & $\operatorname{Rh}(1)$ & $C(105)$ & $C(104)$ & $93.5(5)$ \\
\hline $\operatorname{Rh}(1)$ & C(105) & $C(106)$ & $69.2(5)$ & $C(104)$ & $C(105)$ & $C(106)$ & $111.3(7)$ \\
\hline $\operatorname{Rh}(1)$ & C(105) & $C(115)$ & $119.3(6)$ & $C(104)$ & $\mathrm{C}(105)$ & $C(115)$ & $122.4(7)$ \\
\hline$C(106)$ & $\mathrm{C}(105)$ & $C(115)$ & $123.9(7)$ & $C(510)$ & $\mathrm{C}(511)$ & $\mathrm{C}(512)$ & $120.4(1)$ \\
\hline C(413) & C(414) & $C(409)$ & 121.4(9) & $\operatorname{Rh}(2)$ & $C(203)$ & $C(202)$ & $72.3(5)$ \\
\hline $\operatorname{Rh}(2)$ & $C(203)$ & $C(204)$ & $96.6(6)$ & $C(202)$ & $C(203)$ & $C(204)$ & $113.4(8)$ \\
\hline$C(316)$ & $C(315)$ & $C(305)$ & $121.2(7)$ & $C(316)$ & $C(315)$ & $C(320)$ & $118.7(7)$ \\
\hline$C(305)$ & $\mathrm{C}(315)$ & $C(320)$ & $120.0(7)$ & $\mathrm{C}(207)$ & $\mathrm{C}(208)$ & $C(204)$ & $109.6(8)$ \\
\hline$C(306)$ & $\mathrm{C}(301)$ & $C(302)$ & $100.3(6)$ & C(306) & $C(301)$ & $C(307)$ & $112.0(7)$ \\
\hline$C(302)$ & $\mathrm{C}(301)$ & $C(307)$ & $109.8(8)$ & $C(216)$ & $C(215)$ & $C(205)$ & $120.1(9)$ \\
\hline$C(216)$ & $C(215)$ & $C(220)$ & 118.7(1) & $C(205)$ & $C(215)$ & $C(220)$ & $121.1(8)$ \\
\hline$C(210)$ & $\mathrm{C}(209)$ & $C(202)$ & $122.4(8)$ & $C(210)$ & $\mathrm{C}(209)$ & $C(214)$ & $117.0(9)$ \\
\hline$C(202)$ & $\mathrm{C}(209)$ & $C(214)$ & $120.5(9)$ & C(403) & C(404) & $C(405)$ & $100.8(6)$ \\
\hline C(403) & C(404) & $C(408)$ & $110.1(7)$ & C(405) & $\mathrm{C}(404)$ & $\mathrm{C}(408)$ & 111.1(7) \\
\hline C(401) & $\mathrm{C}(407)$ & $C(408)$ & $110.4(6)$ & $\operatorname{Rh}(3)$ & $C(302)$ & $C(303)$ & $69.1(5)$ \\
\hline $\operatorname{Rh}(3)$ & $\mathrm{C}(302)$ & $C(309)$ & $114.6(6)$ & $\mathrm{C}(303)$ & $\mathrm{C}(302)$ & $\mathrm{C}(309)$ & $124.5(8)$ \\
\hline $\operatorname{Rh}(3)$ & $C(302)$ & $C(301)$ & $94.8(5)$ & $C(303)$ & $\mathrm{C}(302)$ & $C(301)$ & $111.0(7)$ \\
\hline C(309) & $\mathrm{C}(302)$ & $C(301)$ & $123.1(7)$ & $\mathrm{C}(314)$ & $\mathrm{C}(313)$ & $\mathrm{C}(312)$ & $120.7(9)$ \\
\hline$C(515)$ & $C(516)$ & $C(517)$ & $120.5(8)$ & $\operatorname{Rh}(2)$ & $\mathrm{C}(202)$ & $C(203)$ & $68.8(6)$ \\
\hline $\operatorname{Rh}(2)$ & $C(202)$ & $C(209)$ & $118.5(6)$ & $\mathrm{C}(203)$ & $\mathrm{C}(202)$ & $\mathrm{C}(209)$ & $125.1(8)$ \\
\hline $\operatorname{Rh}(2)$ & $C(202)$ & $C(201)$ & $93.9(5)$ & $C(203)$ & $\mathrm{C}(202)$ & $C(201)$ & $111.5(8)$ \\
\hline$C(209)$ & $C(202)$ & $C(201)$ & $121.3(8)$ & $\operatorname{Rh}(2)$ & $C(205)$ & $C(206)$ & $69.8(5)$ \\
\hline $\operatorname{Rh}(2)$ & $C(205)$ & $C(215)$ & $113.7(6)$ & $C(206)$ & $C(205)$ & $C(215)$ & $124.8(8)$ \\
\hline $\operatorname{Rh}(2)$ & $C(205)$ & $C(204)$ & $94.7(5)$ & $C(206)$ & $C(205)$ & $C(204)$ & $110.2(8)$ \\
\hline$C(215)$ & $C(205)$ & $C(204)$ & $123.7(8)$ & $C(115)$ & $\mathrm{C}(120)$ & $\mathrm{C}(119)$ & $121.5(8)$ \\
\hline C(419) & $\mathrm{C}(420)$ & $C(415)$ & 121.1(8) & $C(613)$ & $\mathrm{C}(614)$ & $\mathrm{C}(609)$ & 121.1(8) \\
\hline$C(315)$ & $\mathrm{C}(320)$ & $C(319)$ & $120.3(8)$ & $\mathrm{C}(318)$ & $\mathrm{C}(319)$ & $C(320)$ & $121.4(9)$ \\
\hline$C(203)$ & $C(204)$ & $C(208)$ & $110.8(9)$ & $C(203)$ & $C(204)$ & $C(205)$ & $99.6(7)$ \\
\hline$C(208)$ & $\mathrm{C}(204)$ & $C(205)$ & $112.0(8)$ & $\mathrm{C}(109)$ & $C(110)$ & $C(111)$ & 119.9(1) \\
\hline$C(101)$ & C(107) & $C(108)$ & $110.7(7)$ & $C(613)$ & $C(612)$ & $C(611)$ & $119.5(9)$ \\
\hline$C(616)$ & $\mathrm{C}(617)$ & $C(618)$ & 121.3(9) & $\mathrm{C}(111)$ & $\mathrm{C}(112)$ & $\mathrm{C}(113)$ & $118.6(1)$ \\
\hline$C(520)$ & $\mathrm{C}(519)$ & $C(518)$ & $120.8(1)$ & $C(316)$ & $C(317)$ & $C(318)$ & $120.7(8)$ \\
\hline$C(601)$ & C(607) & $C(608)$ & $109.5(7)$ & $C(116)$ & $C(115)$ & $C(105)$ & $120.3(7)$ \\
\hline$C(116)$ & $C(115)$ & $C(120)$ & $118.0(7)$ & $C(105)$ & $C(115)$ & $C(120)$ & $121.6(7)$ \\
\hline C(410) & C(409) & $C(402)$ & $119.6(8)$ & $C(410)$ & C(409) & $\mathrm{C}(414)$ & 118.3(8) \\
\hline$C(402)$ & C(409) & $C(414)$ & 122.1(8) & $\mathrm{C}(509)$ & $C(514)$ & $C(513)$ & $120.7(1)$ \\
\hline$C(507)$ & $\mathrm{C}(508)$ & $C(504)$ & $110.8(8)$ & $C(218)$ & $\mathrm{C}(219)$ & $C(220)$ & $120.0(1)$ \\
\hline $\operatorname{Rh}(5)$ & $\mathrm{C}(502)$ & $C(503)$ & $69.2(5)$ & $\operatorname{Rh}(5)$ & $\mathrm{C}(502)$ & $\mathrm{C}(509)$ & $117.5(6)$ \\
\hline$C(503)$ & $C(502)$ & $C(509)$ & $123.8(8)$ & $\operatorname{Rh}(5)$ & $\mathrm{C}(502)$ & $C(501)$ & $93.9(5)$ \\
\hline$C(503)$ & $\mathrm{C}(502)$ & $C(501)$ & $111.8(7)$ & $\mathrm{C}(509)$ & $\mathrm{C}(502)$ & $\mathrm{C}(501)$ & $122.4(8)$ \\
\hline C(308) & C(307) & $C(301)$ & $111.5(7)$ & $C(616)$ & $C(615)$ & $C(605)$ & $120.7(7)$ \\
\hline$C(616)$ & $\mathrm{C}(615)$ & $C(620)$ & $117.8(8)$ & $C(605)$ & $C(615)$ & $C(620)$ & $121.4(7)$ \\
\hline$C(207)$ & $\mathrm{C}(201)$ & $C(206)$ & $112.0(8)$ & $C(207)$ & $\mathrm{C}(201)$ & $C(202)$ & $108.4(7)$ \\
\hline C(206) & $\mathrm{C}(201)$ & $C(202)$ & $100.8(7)$ & $\mathrm{C}(118)$ & $\mathrm{C}(119)$ & $C(120)$ & $120.1(8)$ \\
\hline $\operatorname{Rh}(5)$ & $\mathrm{C}(506)$ & $C(505)$ & $72.6(5)$ & $\operatorname{Rh}(5)$ & $C(506)$ & $C(501)$ & $95.8(5)$ \\
\hline$C(505)$ & $\mathrm{C}(506)$ & $C(501)$ & $114.0(7)$ & $C(213)$ & $C(212)$ & $C(211)$ & 119.6(1) \\
\hline $\mathrm{C}(511)$ & $C(512)$ & $C(513)$ & $118.8(9)$ & $C(213)$ & $C(214)$ & $C(209)$ & $121.9(1)$ \\
\hline C(604) & C(608) & $C(607)$ & $109.2(7)$ & $\mathrm{C}(517)$ & $C(518)$ & $\mathrm{C}(519)$ & $117.4(9)$ \\
\hline$C(116)$ & $\mathrm{C}(117)$ & $C(118)$ & $119.5(8)$ & $\mathrm{C}(507)$ & $\mathrm{C}(501)$ & $C(502)$ & $109.7(8)$ \\
\hline$C(507)$ & $\mathrm{C}(501)$ & $C(506)$ & $110.5(7)$ & $\mathrm{C}(502)$ & $\mathrm{C}(501)$ & $C(506)$ & $100.8(6)$ \\
\hline
\end{tabular}




$\begin{array}{llllllll}\mathrm{C}(503) & \mathrm{C}(504) & \mathrm{C}(505) & 100.2(7) & \mathrm{C}(503) & \mathrm{C}(504) & \mathrm{C}(508) & 111.4(8) \\ \mathrm{C}(505) & \mathrm{C}(504) & \mathrm{C}(508) & 109.3(8) & \mathrm{C}(215) & \mathrm{C}(220) & \mathrm{C}(219) & 120.8(1) \\ \mathrm{C}(610) & \mathrm{C}(609) & \mathrm{C}(602) & 121.3(7) & \mathrm{C}(610) & \mathrm{C}(609) & \mathrm{C}(614) & 117.4(8) \\ \mathrm{C}(602) & \mathrm{C}(609) & \mathrm{C}(614) & 121.3(7) & \mathrm{C}(514) & \mathrm{C}(513) & \mathrm{C}(512) & 121.1(1)\end{array}$

Table S9. Bond angles involving hydrogens $\left({ }^{\circ}\right)$

\begin{tabular}{|c|c|c|c|c|c|c|c|}
\hline atom & atom & atom & angle & atom & atom & atom & angle \\
\hline $\operatorname{Rh}(6)$ & $\operatorname{Rh}(5)$ & $\mathrm{Cl}(6)$ & $52.79(7)$ & $\operatorname{Rh}(6)$ & $\operatorname{Rh}(5)$ & $\mathrm{Cl}(5)$ & $52.53(6)$ \\
\hline $\mathrm{Cl}(6)$ & $\operatorname{Rh}(5)$ & $\mathrm{Cl}(5)$ & $84.62(7)$ & $\operatorname{Rh}(6)$ & $\operatorname{Rh}(5)$ & $C(503)$ & $117.8(3)$ \\
\hline $\mathrm{Cl}(6)$ & $\operatorname{Rh}(5)$ & $C(503)$ & $99.5(2)$ & $\mathrm{Cl}(5)$ & $\operatorname{Rh}(5)$ & $C(503)$ & $163.3(2)$ \\
\hline $\operatorname{Rh}(6)$ & $\operatorname{Rh}(5)$ & $C(505)$ & $113.4(2)$ & $\mathrm{Cl}(6)$ & $\operatorname{Rh}(5)$ & $C(505)$ & $154.3(2)$ \\
\hline $\mathrm{Cl}(5)$ & $\operatorname{Rh}(5)$ & $C(505)$ & $103.6(2)$ & $C(503)$ & $\operatorname{Rh}(5)$ & $C(505)$ & $66.1(3)$ \\
\hline $\operatorname{Rh}(6)$ & $\operatorname{Rh}(5)$ & $\mathrm{C}(502)$ & $147.8(2)$ & $\mathrm{Cl}(6)$ & $\operatorname{Rh}(5)$ & $\mathrm{C}(502)$ & $101.9(2)$ \\
\hline $\mathrm{Cl}(5)$ & $\operatorname{Rh}(5)$ & $\mathrm{C}(502)$ & $155.7(3)$ & $C(503)$ & $\operatorname{Rh}(5)$ & $C(502)$ & 39.3(3) \\
\hline$C(505)$ & $\operatorname{Rh}(5)$ & $\mathrm{C}(502)$ & $80.7(3)$ & $\operatorname{Rh}(6)$ & $\operatorname{Rh}(5)$ & $C(506)$ & $141.5(2)$ \\
\hline $\mathrm{Cl}(6)$ & $\operatorname{Rh}(5)$ & $\mathrm{C}(506)$ & $163.9(2)$ & $\mathrm{Cl}(5)$ & $\operatorname{Rh}(5)$ & $C(506)$ & $100.6(3)$ \\
\hline$C(503)$ & $\operatorname{Rh}(5)$ & $\mathrm{C}(506)$ & $79.8(3)$ & $C(505)$ & $\operatorname{Rh}(5)$ & $C(506)$ & $39.3(3)$ \\
\hline$C(502)$ & $\operatorname{Rh}(5)$ & $\mathrm{C}(506)$ & $67.3(3)$ & $\operatorname{Rh}(1)$ & $\operatorname{Rh}(2)$ & $\mathrm{Cl}(2)$ & $53.16(6)$ \\
\hline $\mathrm{Rh}(1)$ & $\operatorname{Rh}(2)$ & $\mathrm{Cl}(1)$ & $52.70(6)$ & $\mathrm{Cl}(2)$ & $\operatorname{Rh}(2)$ & $\mathrm{Cl}(1)$ & $82.7(1)$ \\
\hline $\operatorname{Rh}(1)$ & $\operatorname{Rh}(2)$ & $C(206)$ & $113.8(2)$ & $\mathrm{Cl}(2)$ & $\operatorname{Rh}(2)$ & $C(206)$ & $162.5(2)$ \\
\hline $\mathrm{Cl}(1)$ & $\operatorname{Rh}(2)$ & $\mathrm{C}(206)$ & $98.0(3)$ & $\operatorname{Rh}(1)$ & $\operatorname{Rh}(2)$ & $C(203)$ & $139.9(3)$ \\
\hline $\mathrm{Cl}(2)$ & $\operatorname{Rh}(2)$ & $C(203)$ & $103.3(3)$ & $\mathrm{Cl}(1)$ & $\operatorname{Rh}(2)$ & $C(203)$ & $167.0(3)$ \\
\hline$C(206)$ & $\operatorname{Rh}(2)$ & $C(203)$ & $79.8(4)$ & $\operatorname{Rh}(1)$ & $\operatorname{Rh}(2)$ & $\mathrm{C}(202)$ & $109.3(2)$ \\
\hline $\mathrm{Cl}(2)$ & $\operatorname{Rh}(2)$ & $\mathrm{C}(202)$ & $104.4(2)$ & $\mathrm{Cl}(1)$ & $\operatorname{Rh}(2)$ & $C(202)$ & $151.3(3)$ \\
\hline$C(206)$ & $\operatorname{Rh}(2)$ & $C(202)$ & $67.0(3)$ & $C(203)$ & $\operatorname{Rh}(2)$ & $C(202)$ & $38.8(4)$ \\
\hline $\operatorname{Rh}(1)$ & $\operatorname{Rh}(2)$ & $C(205)$ & $145.5(2)$ & $\mathrm{Cl}(2)$ & $\operatorname{Rh}(2)$ & $C(205)$ & $158.1(3)$ \\
\hline $\mathrm{Cl}(1)$ & $\operatorname{Rh}(2)$ & $C(205)$ & $102.6(3)$ & C(206) & $\operatorname{Rh}(2)$ & $\mathrm{C}(205)$ & $38.8(3)$ \\
\hline$C(203)$ & $\operatorname{Rh}(2)$ & $C(205)$ & $67.6(4)$ & $C(202)$ & $\operatorname{Rh}(2)$ & $C(205)$ & $81.3(4)$ \\
\hline $\mathrm{Rh}(4)$ & $\operatorname{Rh}(3)$ & $\mathrm{Cl}(3)$ & $51.55(7)$ & $\operatorname{Rh}(4)$ & $\operatorname{Rh}(3)$ & $\mathrm{Cl}(4)$ & $51.10(6)$ \\
\hline $\mathrm{Cl}(3)$ & $\operatorname{Rh}(3)$ & $\mathrm{Cl}(4)$ & $84.60(9)$ & $\operatorname{Rh}(4)$ & $\operatorname{Rh}(3)$ & $C(303)$ & $138.6(2)$ \\
\hline $\mathrm{Cl}(3)$ & $\operatorname{Rh}(3)$ & $\mathrm{C}(303)$ & $169.0(2)$ & $\mathrm{Cl}(4)$ & $\operatorname{Rh}(3)$ & $C(303)$ & $100.2(2)$ \\
\hline $\operatorname{Rh}(4)$ & $\operatorname{Rh}(3)$ & $\mathrm{C}(306)$ & $120.5(2)$ & $\mathrm{Cl}(3)$ & $\operatorname{Rh}(3)$ & C(306) & $97.8(3)$ \\
\hline $\mathrm{Cl}(4)$ & $\operatorname{Rh}(3)$ & $\mathrm{C}(306)$ & $165.9(2)$ & $C(303)$ & $\operatorname{Rh}(3)$ & $C(306)$ & $79.9(3)$ \\
\hline $\operatorname{Rh}(4)$ & $\operatorname{Rh}(3)$ & $C(305)$ & $150.8(2)$ & $\mathrm{Cl}(3)$ & $\operatorname{Rh}(3)$ & $C(305)$ & $103.8(2)$ \\
\hline $\mathrm{Cl}(4)$ & $\operatorname{Rh}(3)$ & $\mathrm{C}(305)$ & $153.7(2)$ & $C(303)$ & $\operatorname{Rh}(3)$ & $C(305)$ & $67.6(3)$ \\
\hline$C(306)$ & $\operatorname{Rh}(3)$ & $\mathrm{C}(305)$ & 39.0(3) & $\operatorname{Rh}(4)$ & $\operatorname{Rh}(3)$ & $\mathrm{C}(302)$ & $112.3(2)$ \\
\hline $\mathrm{Cl}(3)$ & $\operatorname{Rh}(3)$ & $\mathrm{C}(302)$ & $149.6(2)$ & $\mathrm{Cl}(4)$ & $\operatorname{Rh}(3)$ & $C(302)$ & $104.6(2)$ \\
\hline$C(303)$ & $\operatorname{Rh}(3)$ & $\mathrm{C}(302)$ & 38.7(3) & $C(306)$ & $\operatorname{Rh}(3)$ & $C(302)$ & $66.6(3)$ \\
\hline$C(305)$ & $\operatorname{Rh}(3)$ & $\mathrm{C}(302)$ & $80.8(3)$ & $\operatorname{Rh}(3)$ & $\operatorname{Rh}(4)$ & $\mathrm{Cl}(3)$ & $51.58(7)$ \\
\hline $\operatorname{Rh}(3)$ & $\operatorname{Rh}(4)$ & $\mathrm{Cl}(4)$ & $52.18(6)$ & $\mathrm{Cl}(3)$ & $\operatorname{Rh}(4)$ & $\mathrm{Cl}(4)$ & $85.41(8)$ \\
\hline $\operatorname{Rh}(3)$ & $\operatorname{Rh}(4)$ & $\mathrm{C}(403)$ & $143.6(2)$ & $\mathrm{Cl}(3)$ & $\operatorname{Rh}(4)$ & C(403) & $101.5(2)$ \\
\hline $\mathrm{Cl}(4)$ & $\operatorname{Rh}(4)$ & C(403) & $162.3(2)$ & $\operatorname{Rh}(3)$ & $\operatorname{Rh}(4)$ & $C(405)$ & $145.2(2)$ \\
\hline $\mathrm{Cl}(3)$ & $\operatorname{Rh}(4)$ & C(405) & $159.9(2)$ & $\mathrm{Cl}(4)$ & $\operatorname{Rh}(4)$ & $C(405)$ & $100.4(2)$ \\
\hline$C(403)$ & $\operatorname{Rh}(4)$ & $C(405)$ & $67.8(3)$ & $\operatorname{Rh}(3)$ & $\operatorname{Rh}(4)$ & C(406) & $116.7(2)$ \\
\hline $\mathrm{Cl}(3)$ & $\mathrm{Rh}(4)$ & $C(406)$ & $159.4(2)$ & $\mathrm{Cl}(4)$ & $\operatorname{Rh}(4)$ & $\mathrm{C}(406)$ & $99.6(2)$ \\
\hline$C(403)$ & $\operatorname{Rh}(4)$ & $\mathrm{C}(406)$ & $79.7(3)$ & $C(405)$ & $\operatorname{Rh}(4)$ & C(406) & $38.9(3)$ \\
\hline $\operatorname{Rh}(3)$ & $\operatorname{Rh}(4)$ & $\mathrm{C}(402)$ & $114.7(2)$ & $\mathrm{Cl}(3)$ & $\operatorname{Rh}(4)$ & $\mathrm{C}(402)$ & $101.2(2)$ \\
\hline $\mathrm{Cl}(4)$ & $\operatorname{Rh}(4)$ & $\mathrm{C}(402)$ & $155.9(2)$ & $C(403)$ & $\operatorname{Rh}(4)$ & $\mathrm{C}(402)$ & $39.1(3)$ \\
\hline$C(405)$ & $\operatorname{Rh}(4)$ & $\mathrm{C}(402)$ & 81.3(3) & C(406) & $\operatorname{Rh}(4)$ & C(402) & $66.6(3)$ \\
\hline $\operatorname{Rh}(5)$ & $\operatorname{Rh}(6)$ & $\mathrm{Cl}(6)$ & $52.88(6)$ & $\operatorname{Rh}(5)$ & $\operatorname{Rh}(6)$ & $\mathrm{Cl}(5)$ & $52.99(6)$ \\
\hline
\end{tabular}




\begin{tabular}{|c|c|c|c|c|c|c|c|}
\hline $\mathrm{Cl}(6)$ & $\operatorname{Rh}(6)$ & $\mathrm{Cl}(5)$ & $84.99(9)$ & $\mathrm{Rh}(5)$ & $\operatorname{Rh}(6)$ & $C(605)$ & $111.6(2)$ \\
\hline $\mathrm{Cl}(6)$ & $\operatorname{Rh}(6)$ & $\mathrm{C}(605)$ & $102.1(2)$ & $\mathrm{Cl}(5)$ & $\mathrm{Rh}(6)$ & $C(605)$ & $153.6(2)$ \\
\hline $\operatorname{Rh}(5)$ & $\operatorname{Rh}(6)$ & $C(602)$ & $146.6(2)$ & $\mathrm{Cl}(6)$ & $\mathrm{Rh}(6)$ & $C(602)$ & $157.8(2)$ \\
\hline $\mathrm{Cl}(5)$ & $\operatorname{Rh}(6)$ & $C(602)$ & $102.2(2)$ & $C(605)$ & $\mathrm{Rh}(6)$ & $\mathrm{C}(602)$ & $80.8(3)$ \\
\hline $\operatorname{Rh}(5)$ & $\operatorname{Rh}(6)$ & $C(603)$ & $115.9(2)$ & $\mathrm{Cl}(6)$ & $\operatorname{Rh}(6)$ & $C(603)$ & $161.5(2)$ \\
\hline $\mathrm{Cl}(5)$ & $\operatorname{Rh}(6)$ & $C(603)$ & $99.1(2)$ & $C(605)$ & $\operatorname{Rh}(6)$ & $C(603)$ & $66.7(3)$ \\
\hline$C(602)$ & $\operatorname{Rh}(6)$ & $C(603)$ & $38.8(3)$ & $\operatorname{Rh}(5)$ & $\operatorname{Rh}(6)$ & $\mathrm{C}(606)$ & $140.8(2)$ \\
\hline $\mathrm{Cl}(6)$ & $\operatorname{Rh}(6)$ & $C(606)$ & $100.8(2)$ & $\mathrm{Cl}(5)$ & $\operatorname{Rh}(6)$ & $C(606)$ & $165.0(2)$ \\
\hline$C(605)$ & $\operatorname{Rh}(6)$ & $C(606)$ & $38.9(3)$ & $C(602)$ & $\operatorname{Rh}(6)$ & $C(606)$ & $67.5(3)$ \\
\hline$C(603)$ & $\mathrm{Rh}(6)$ & $C(606)$ & 79.9(3) & $\operatorname{Rh}(2)$ & $\mathrm{Rh}(1)$ & $\mathrm{Cl}(2)$ & $53.13(6)$ \\
\hline $\operatorname{Rh}(2)$ & $\operatorname{Rh}(1)$ & $\mathrm{Cl}(1)$ & $53.20(6)$ & $\mathrm{Cl}(2)$ & $\operatorname{Rh}(1)$ & $\mathrm{Cl}(1)$ & $83.0(1)$ \\
\hline $\operatorname{Rh}(2)$ & $\operatorname{Rh}(1)$ & $C(102)$ & $146.3(2)$ & $\mathrm{Cl}(2)$ & $\mathrm{Rh}(1)$ & $\mathrm{C}(102)$ & $106.3(2)$ \\
\hline $\mathrm{Cl}(1)$ & $\operatorname{Rh}(1)$ & $C(102)$ & $159.8(2)$ & $\mathrm{Rh}(2)$ & $\mathrm{Rh}(1)$ & C(103) & $112.3(2)$ \\
\hline $\mathrm{Cl}(2)$ & $\operatorname{Rh}(1)$ & $\mathrm{C}(103)$ & $99.2(2)$ & $\mathrm{Cl}(1)$ & $\operatorname{Rh}(1)$ & $C(103)$ & $159.6(2)$ \\
\hline$C(102)$ & $\mathrm{Rh}(1)$ & $\mathrm{C}(103)$ & $38.4(3)$ & $\mathrm{Rh}(2)$ & $\mathrm{Rh}(1)$ & C(106) & $136.8(2)$ \\
\hline $\mathrm{Cl}(2)$ & $\operatorname{Rh}(1)$ & C(106) & $169.9(3)$ & $\mathrm{Cl}(1)$ & $\operatorname{Rh}(1)$ & C(106) & $102.3(3)$ \\
\hline$C(102)$ & $\operatorname{Rh}(1)$ & C(106) & $65.9(3)$ & $C(103)$ & $\operatorname{Rh}(1)$ & C(106) & $78.9(3)$ \\
\hline $\mathrm{Rh}(2)$ & $\operatorname{Rh}(1)$ & $C(105)$ & $105.5(2)$ & $\mathrm{Cl}(2)$ & $\mathrm{Rh}(1)$ & $C(105)$ & $149.7(2)$ \\
\hline $\mathrm{Cl}(1)$ & $\operatorname{Rh}(1)$ & $C(105)$ & $101.1(2)$ & $C(102)$ & $\operatorname{Rh}(1)$ & $C(105)$ & $80.0(3)$ \\
\hline$C(103)$ & $\operatorname{Rh}(1)$ & $C(105)$ & $67.0(3)$ & $C(106)$ & $\operatorname{Rh}(1)$ & $C(105)$ & $38.3(3)$ \\
\hline $\operatorname{Rh}(2)$ & $\mathrm{Cl}(2)$ & $\operatorname{Rh}(1)$ & $73.72(8)$ & $\operatorname{Rh}(3)$ & $\mathrm{Cl}(3)$ & $\operatorname{Rh}(4)$ & $76.86(8)$ \\
\hline $\operatorname{Rh}(3)$ & $\mathrm{Cl}(4)$ & $\operatorname{Rh}(4)$ & $76.72(7)$ & $\operatorname{Rh}(5)$ & $\mathrm{Cl}(6)$ & $\operatorname{Rh}(6)$ & $74.33(7)$ \\
\hline $\operatorname{Rh}(5)$ & $\mathrm{Cl}(5)$ & $\operatorname{Rh}(6)$ & $74.48(7)$ & $\operatorname{Rh}(2)$ & $\mathrm{Cl}(1)$ & $\mathrm{Rh}(1)$ & $74.10(8)$ \\
\hline $\mathrm{C}(509)$ & $C(510)$ & $\mathrm{H}(509)$ & $119.8(1)$ & $C(511)$ & $C(510)$ & $\mathrm{H}(509)$ & $120.0(1)$ \\
\hline $\operatorname{Rh}(3)$ & $C(303)$ & $C(304)$ & $95.0(5)$ & $\operatorname{Rh}(3)$ & $C(303)$ & $C(302)$ & $72.2(5)$ \\
\hline $\operatorname{Rh}(3)$ & $C(303)$ & $\mathrm{H}(302)$ & $120.7(8)$ & $C(304)$ & $C(303)$ & $\mathrm{H}(302)$ & $120.7(1)$ \\
\hline $\mathrm{C}(302)$ & $\mathrm{C}(303)$ & $\mathrm{H}(302)$ & $120.6(1)$ & $\operatorname{Rh}(1)$ & $C(102)$ & C(109) & $122.3(6)$ \\
\hline $\mathrm{Rh}(1)$ & $\mathrm{C}(102)$ & $\mathrm{C}(103)$ & $68.2(4)$ & $\mathrm{Rh}(1)$ & $C(102)$ & C(101) & $95.0(5)$ \\
\hline C(309) & $C(314)$ & $\mathrm{H}(313)$ & 119.1(1) & $C(313)$ & $C(314)$ & $\mathrm{H}(313)$ & $119.9(1)$ \\
\hline $\mathrm{C}(605)$ & $C(604)$ & $\mathrm{H}(603)$ & 111.1(9) & $C(603)$ & $C(604)$ & $\mathrm{H}(603)$ & 111.3(9) \\
\hline $\mathrm{C}(608)$ & $\mathrm{C}(604)$ & $\mathrm{H}(603)$ & 111.8(9) & $\mathrm{Rh}(4)$ & C(403) & $C(402)$ & $72.0(4)$ \\
\hline $\operatorname{Rh}(4)$ & $\mathrm{C}(403)$ & $C(404)$ & $95.4(5)$ & $\operatorname{Rh}(4)$ & $C(403)$ & $\mathrm{H}(402)$ & $120.6(7)$ \\
\hline $\mathrm{C}(402)$ & $C(403)$ & $\mathrm{H}(402)$ & $120.6(9)$ & $C(404)$ & $C(403)$ & $\mathrm{H}(402)$ & 121.1(9) \\
\hline $\mathrm{C}(212)$ & $\mathrm{C}(213)$ & $\mathrm{H}(212)$ & $120.4(1)$ & $C(214)$ & $C(213)$ & $\mathrm{H}(212)$ & $119.9(1)$ \\
\hline C(315) & $C(316)$ & $\mathrm{H}(314)$ & $120.0(1)$ & $C(317)$ & $C(316)$ & $\mathrm{H}(314)$ & $120.0(1)$ \\
\hline C(406) & $\mathrm{C}(401)$ & $\mathrm{H}(401)$ & 111.6(9) & $C(402)$ & $C(401)$ & $\mathrm{H}(401)$ & $111.5(9)$ \\
\hline C(407) & $\mathrm{C}(401)$ & $\mathrm{H}(401)$ & 112.2(9) & $\operatorname{Rh}(4)$ & $C(405)$ & C(406) & $70.0(4)$ \\
\hline $\operatorname{Rh}(4)$ & $C(405)$ & $C(415)$ & $117.5(5)$ & $\operatorname{Rh}(4)$ & $C(405)$ & $\mathrm{C}(404)$ & $94.0(5)$ \\
\hline C(103) & C(104) & $\mathrm{H}(103)$ & 111.7(9) & $C(108)$ & $C(104)$ & $\mathrm{H}(103)$ & $112.2(1)$ \\
\hline C(105) & C(104) & $\mathrm{H}(103)$ & 111.3(9) & $C(115)$ & $C(116)$ & $\mathrm{H}(114)$ & 119.3(1) \\
\hline C(117) & $C(116)$ & $\mathrm{H}(114)$ & $119.5(1)$ & $C(411)$ & $C(410)$ & $\mathrm{H}(409)$ & $120.3(1)$ \\
\hline C(409) & $C(410)$ & $\mathrm{H}(409)$ & 119.9(1) & $C(412)$ & $C(413)$ & $\mathrm{H}(412)$ & $120.0(1)$ \\
\hline C(414) & $C(413)$ & $\mathrm{H}(412)$ & 119.7(1) & $C(617)$ & $C(616)$ & $\mathrm{H}(614)$ & $119.7(1)$ \\
\hline$C(615)$ & $C(616)$ & $\mathrm{H}(614)$ & 119.7(1) & $\operatorname{Rh}(6)$ & $C(605)$ & $C(604)$ & $94.6(5)$ \\
\hline $\operatorname{Rh}(6)$ & $C(605)$ & $C(606)$ & $69.1(4)$ & $\operatorname{Rh}(6)$ & $C(605)$ & $C(615)$ & $115.4(5)$ \\
\hline$C(404)$ & $\mathrm{C}(408)$ & $\mathrm{H}(408)$ & $109.9(9)$ & $C(407)$ & $C(408)$ & $\mathrm{H}(408)$ & $109.2(1)$ \\
\hline$C(404)$ & C(408) & $\mathrm{H}(407)$ & 109.3(9) & $C(407)$ & $C(408)$ & $\mathrm{H}(407)$ & $109.7(1)$ \\
\hline $\mathrm{H}(408)$ & C(408) & $\mathrm{H}(407)$ & $109.5(1)$ & $C(602)$ & $C(601)$ & $\mathrm{H}(601)$ & 111.3(9) \\
\hline$C(606)$ & $C(601)$ & $\mathrm{H}(601)$ & $111.8(1)$ & $C(607)$ & $C(601)$ & $\mathrm{H}(601)$ & $111.9(1)$ \\
\hline $\operatorname{Rh}(3)$ & C(306) & $\mathrm{C}(305)$ & 72.1(4) & $\operatorname{Rh}(3)$ & $C(306)$ & $C(301)$ & $97.2(5)$ \\
\hline $\operatorname{Rh}(3)$ & C(306) & $\mathrm{H}(304)$ & $120.8(7)$ & $C(305)$ & $C(306)$ & $\mathrm{H}(304)$ & $120.4(9)$ \\
\hline $\mathrm{C}(301)$ & $C(306)$ & $\mathrm{H}(304)$ & 120.9(9) & $C(418)$ & $C(417)$ & $\mathrm{H}(415)$ & $119.4(1)$ \\
\hline C(416) & C(417) & $\mathrm{H}(415)$ & 119.1(1) & $\operatorname{Rh}(4)$ & $C(406)$ & $\mathrm{C}(401)$ & $96.4(5)$ \\
\hline
\end{tabular}




\begin{tabular}{|c|c|c|c|c|c|c|c|}
\hline $\operatorname{Rh}(4)$ & $C(406)$ & $C(405)$ & $71.0(4)$ & $\mathrm{Rh}(4)$ & $C(406)$ & $\mathrm{H}(404)$ & $120.3(7)$ \\
\hline$C(401)$ & C(406) & $\mathrm{H}(404)$ & $120.9(9)$ & $C(405)$ & $\mathrm{C}(406)$ & $\mathrm{H}(404)$ & 120.1(9) \\
\hline$C(303)$ & C(304) & $\mathrm{H}(303)$ & $111.9(1)$ & $C(305)$ & $\mathrm{C}(304)$ & $\mathrm{H}(303)$ & $111.4(1)$ \\
\hline$C(308)$ & $\mathrm{C}(304)$ & $\mathrm{H}(303)$ & 111.7(1) & $C(109)$ & $\mathrm{C}(114)$ & $\mathrm{H}(113)$ & 119.9(1) \\
\hline$C(113)$ & C(114) & $\mathrm{H}(113)$ & $120.2(1)$ & C(319) & $\mathrm{C}(318)$ & $\mathrm{H}(316)$ & $120.8(1)$ \\
\hline$C(317)$ & C(318) & $\mathrm{H}(316)$ & $120.4(1)$ & $\operatorname{Rh}(5)$ & $C(503)$ & $C(502)$ & $71.4(5)$ \\
\hline $\operatorname{Rh}(5)$ & $\mathrm{C}(503)$ & $C(504)$ & $97.1(5)$ & $\operatorname{Rh}(5)$ & $C(503)$ & $\mathrm{H}(502)$ & $120.5(8)$ \\
\hline$C(502)$ & $C(503)$ & $\mathrm{H}(502)$ & $120.5(1)$ & $C(504)$ & $\mathrm{C}(503)$ & $\mathrm{H}(502)$ & $120.8(1)$ \\
\hline$C(611)$ & $C(610)$ & $\mathrm{H}(609)$ & $120.0(1)$ & $\mathrm{C}(609)$ & $\mathrm{C}(610)$ & $\mathrm{H}(609)$ & $119.5(1)$ \\
\hline $\mathrm{Rh}(4)$ & $\mathrm{C}(402)$ & $C(403)$ & $69.0(4)$ & $\mathrm{Rh}(4)$ & $C(402)$ & $\mathrm{C}(401)$ & $94.8(5)$ \\
\hline $\operatorname{Rh}(4)$ & $\mathrm{C}(402)$ & $C(409)$ & $115.5(5)$ & $\operatorname{Rh}(6)$ & $C(602)$ & $C(601)$ & $94.3(5)$ \\
\hline $\operatorname{Rh}(6)$ & $C(602)$ & $C(603)$ & $69.5(5)$ & $\operatorname{Rh}(6)$ & $C(602)$ & $\mathrm{C}(609)$ & $115.4(5)$ \\
\hline$C(410)$ & $\mathrm{C}(411)$ & $\mathrm{H}(410)$ & $120.2(2)$ & $C(412)$ & $\mathrm{C}(411)$ & $\mathrm{H}(410)$ & $119.4(2)$ \\
\hline$C(516)$ & $\mathrm{C}(517)$ & $\mathrm{H}(515)$ & 118.3(1) & $C(518)$ & $C(517)$ & $\mathrm{H}(515)$ & $118.5(1)$ \\
\hline$C(216)$ & $C(217)$ & $\mathrm{H}(215)$ & $120.7(2)$ & $C(218)$ & $C(217)$ & $\mathrm{H}(215)$ & $118.9(2)$ \\
\hline$C(618)$ & $\mathrm{C}(619)$ & $\mathrm{H}(617)$ & $119.6(1)$ & $C(620)$ & C(619) & $\mathrm{H}(617)$ & 119.9(1) \\
\hline$C(417)$ & C(418) & $\mathrm{H}(416)$ & $120.9(1)$ & C(419) & $\mathrm{C}(418)$ & $\mathrm{H}(416)$ & $121.0(1)$ \\
\hline$C(417)$ & $C(416)$ & $\mathrm{H}(414)$ & $119.6(1)$ & $C(415)$ & $C(416)$ & $\mathrm{H}(414)$ & 119.1(1) \\
\hline$C(208)$ & $C(207)$ & $\mathrm{H}(206)$ & $109.2(1)$ & $C(201)$ & $C(207)$ & $\mathrm{H}(206)$ & $109.3(1)$ \\
\hline$C(208)$ & $C(207)$ & $\mathrm{H}(205)$ & $109.9(1)$ & $C(201)$ & $C(207)$ & $\mathrm{H}(205)$ & $109.8(1)$ \\
\hline $\mathrm{H}(206)$ & $C(207)$ & $\mathrm{H}(205)$ & $109.5(1)$ & $C(614)$ & $C(613)$ & $\mathrm{H}(612)$ & $120.3(1)$ \\
\hline$C(612)$ & $C(613)$ & $\mathrm{H}(612)$ & $120.0(1)$ & $C(619)$ & $\mathrm{C}(618)$ & $\mathrm{H}(616)$ & $121.2(1)$ \\
\hline$C(617)$ & $C(618)$ & $\mathrm{H}(616)$ & $120.4(1)$ & C(309) & $\mathrm{C}(310)$ & $\mathrm{H}(309)$ & $119.5(1)$ \\
\hline$C(311)$ & $C(310)$ & $\mathrm{H}(309)$ & $119.6(1)$ & $\operatorname{Rh}(1)$ & $C(103)$ & $C(102)$ & $73.4(5)$ \\
\hline $\operatorname{Rh}(1)$ & $C(103)$ & C(104) & $95.6(5)$ & $\operatorname{Rh}(1)$ & $\mathrm{C}(103)$ & $\mathrm{H}(102)$ & $120.3(8)$ \\
\hline$C(102)$ & $C(103)$ & $\mathrm{H}(102)$ & $120.4(1)$ & $C(104)$ & C(103) & $\mathrm{H}(102)$ & $120.8(9)$ \\
\hline$C(310)$ & $\mathrm{C}(311)$ & $\mathrm{H}(310)$ & $120.5(1)$ & $C(312)$ & $\mathrm{C}(311)$ & $\mathrm{H}(310)$ & $120.1(1)$ \\
\hline $\operatorname{Rh}(3)$ & $C(305)$ & C(306) & $68.9(4)$ & $\operatorname{Rh}(3)$ & $C(305)$ & $C(304)$ & $93.2(5)$ \\
\hline $\operatorname{Rh}(3)$ & $C(305)$ & $C(315)$ & $120.0(5)$ & $\operatorname{Rh}(2)$ & $C(206)$ & $C(205)$ & $71.4(5)$ \\
\hline $\operatorname{Rh}(2)$ & $C(206)$ & $\mathrm{C}(201)$ & $96.5(5)$ & $\operatorname{Rh}(2)$ & C(206) & $\mathrm{H}(204)$ & $120.3(8)$ \\
\hline$C(205)$ & $C(206)$ & $\mathrm{H}(204)$ & $120.0(1)$ & $C(201)$ & $C(206)$ & $\mathrm{H}(204)$ & $120.4(1)$ \\
\hline$C(102)$ & $\mathrm{C}(101)$ & $\mathrm{H}(101)$ & $111.4(1)$ & $C(106)$ & $\mathrm{C}(101)$ & $\mathrm{H}(101)$ & $111.2(1)$ \\
\hline$C(107)$ & $\mathrm{C}(101)$ & $\mathrm{H}(101)$ & $111.8(1)$ & $C(217)$ & $C(216)$ & $\mathrm{H}(214)$ & $120.4(1)$ \\
\hline$C(215)$ & $C(216)$ & $\mathrm{H}(214)$ & $120.3(1)$ & $C(110)$ & $\mathrm{C}(111)$ & $\mathrm{H}(110)$ & $118.8(1)$ \\
\hline$C(112)$ & $\mathrm{C}(111)$ & $\mathrm{H}(110)$ & $119.0(1)$ & $C(217)$ & $\mathrm{C}(218)$ & $\mathrm{H}(216)$ & $120.2(2)$ \\
\hline $\mathrm{C}(219)$ & $\mathrm{C}(218)$ & $H(216)$ & $119.0(2)$ & C(418) & $\mathrm{C}(419)$ & $\mathrm{H}(417)$ & $119.7(1)$ \\
\hline$C(420)$ & $\mathrm{C}(419)$ & $\mathrm{H}(417)$ & $119.5(1)$ & C(304) & $\mathrm{C}(308)$ & $\mathrm{H}(308)$ & $109.7(1)$ \\
\hline C(307) & $\mathrm{C}(308)$ & $\mathrm{H}(308)$ & $110.1(1)$ & C(304) & $\mathrm{C}(308)$ & $\mathrm{H}(307)$ & $110.0(1)$ \\
\hline C(307) & $\mathrm{C}(308)$ & $\mathrm{H}(307)$ & $110.0(1)$ & $\mathrm{H}(308)$ & $\mathrm{C}(308)$ & $\mathrm{H}(307)$ & $109.5(1)$ \\
\hline$C(311)$ & $C(312)$ & $\mathrm{H}(311)$ & 119.7(1) & C(313) & $\mathrm{C}(312)$ & $\mathrm{H}(311)$ & $120.4(1)$ \\
\hline$C(119)$ & $\mathrm{C}(118)$ & $\mathrm{H}(116)$ & $120.4(1)$ & $\mathrm{C}(117)$ & C(118) & $\mathrm{H}(116)$ & 119.9(1) \\
\hline $\operatorname{Rh}(6)$ & C(603) & $\mathrm{C}(604)$ & $96.6(5)$ & $\operatorname{Rh}(6)$ & $C(603)$ & $\mathrm{C}(602)$ & $71.7(5)$ \\
\hline $\operatorname{Rh}(6)$ & $C(603)$ & $\mathrm{H}(602)$ & $120.5(8)$ & $C(604)$ & $C(603)$ & $\mathrm{H}(602)$ & 121.2(9) \\
\hline$C(602)$ & $C(603)$ & $\mathrm{H}(602)$ & $120.2(9)$ & $C(508)$ & $\mathrm{C}(507)$ & $\mathrm{H}(506)$ & $109.6(1)$ \\
\hline $\mathrm{C}(501)$ & $\mathrm{C}(507)$ & $\mathrm{H}(506)$ & $109.1(1)$ & $C(508)$ & $\mathrm{C}(507)$ & $\mathrm{H}(505)$ & $109.8(1)$ \\
\hline $\mathrm{C}(501)$ & $\mathrm{C}(507)$ & $\mathrm{H}(505)$ & $109.9(1)$ & $\mathrm{H}(506)$ & $\mathrm{C}(507)$ & $\mathrm{H}(505)$ & $109.5(1)$ \\
\hline C(619) & $\mathrm{C}(620)$ & $\mathrm{H}(618)$ & $119.6(1)$ & $C(615)$ & $\mathrm{C}(620)$ & $\mathrm{H}(618)$ & 119.1(1) \\
\hline $\operatorname{Rh}(6)$ & C(606) & $C(605)$ & $72.0(4)$ & $\operatorname{Rh}(6)$ & C(606) & $\mathrm{C}(601)$ & $95.0(5)$ \\
\hline $\operatorname{Rh}(6)$ & $\mathrm{C}(606)$ & $\mathrm{H}(604)$ & $120.7(8)$ & $C(605)$ & C(606) & $\mathrm{H}(604)$ & $120.9(9)$ \\
\hline$C(601)$ & $\mathrm{C}(606)$ & $\mathrm{H}(604)$ & $121.0(1)$ & $\operatorname{Rh}(5)$ & $C(505)$ & $\mathrm{C}(515)$ & $115.1(6)$ \\
\hline $\operatorname{Rh}(5)$ & $C(505)$ & $\mathrm{C}(506)$ & $68.2(5)$ & $\operatorname{Rh}(5)$ & $C(505)$ & $C(504)$ & $95.3(5)$ \\
\hline$C(211)$ & $C(210)$ & $\mathrm{H}(209)$ & $119.8(1)$ & C(209) & $C(210)$ & $\mathrm{H}(209)$ & 119.1(1) \\
\hline$C(104)$ & $\mathrm{C}(108)$ & $\mathrm{H}(108)$ & $109.7(1)$ & $C(107)$ & $\mathrm{C}(108)$ & $\mathrm{H}(108)$ & $109.6(1)$ \\
\hline
\end{tabular}




\begin{tabular}{|c|c|c|c|c|c|c|c|}
\hline$C(104)$ & $C(108)$ & $\mathrm{H}(107)$ & $109.4(1)$ & $C(107)$ & $\mathrm{C}(108)$ & $\mathrm{H}(107)$ & $109.5(1)$ \\
\hline H(108) & $\mathrm{C}(108)$ & $\mathrm{H}(107)$ & $109.5(1)$ & $\mathrm{C}(413)$ & $\mathrm{C}(412)$ & $\mathrm{H}(411)$ & $119.6(1)$ \\
\hline C(411) & C(412) & $\mathrm{H}(411)$ & $120.5(1)$ & $C(610)$ & $\mathrm{C}(611)$ & $\mathrm{H}(610)$ & 119.1(1) \\
\hline$C(612)$ & $\mathrm{C}(611)$ & $\mathrm{H}(610)$ & $119.3(1)$ & $\operatorname{Rh}(1)$ & $\mathrm{C}(106)$ & $C(101)$ & $97.1(6)$ \\
\hline $\mathrm{Rh}(1)$ & $\mathrm{C}(106)$ & $C(105)$ & $72.5(5)$ & $\operatorname{Rh}(1)$ & C(106) & $\mathrm{H}(104)$ & $120.3(9)$ \\
\hline$C(101)$ & C(106) & $\mathrm{H}(104)$ & $120.7(1)$ & $C(105)$ & $\mathrm{C}(106)$ & $\mathrm{H}(104)$ & $120.1(1)$ \\
\hline $\mathrm{C}(515)$ & $C(520)$ & $\mathrm{H}(518)$ & $119.0(1)$ & $\mathrm{C}(519)$ & $\mathrm{C}(520)$ & $\mathrm{H}(518)$ & $119.4(1)$ \\
\hline$C(114)$ & C(113) & $\mathrm{H}(112)$ & 119.4(1) & $C(112)$ & $\mathrm{C}(113)$ & $\mathrm{H}(112)$ & $119.4(1)$ \\
\hline$C(210)$ & $C(211)$ & $\mathrm{H}(210)$ & $119.5(1)$ & $C(212)$ & $\mathrm{C}(211)$ & $\mathrm{H}(210)$ & $120.0(1)$ \\
\hline $\mathrm{Rh}(1)$ & $C(105)$ & C(104) & $93.5(5)$ & $\mathrm{Rh}(1)$ & $C(105)$ & $\mathrm{C}(106)$ & $69.2(5)$ \\
\hline $\operatorname{Rh}(1)$ & $C(105)$ & $C(115)$ & $119.3(6)$ & $C(510)$ & $\mathrm{C}(511)$ & $\mathrm{H}(510)$ & $119.8(1)$ \\
\hline$C(512)$ & $\mathrm{C}(511)$ & $\mathrm{H}(510)$ & $119.8(1)$ & $\mathrm{C}(413)$ & $\mathrm{C}(414)$ & $\mathrm{H}(413)$ & 119.4(1) \\
\hline C(409) & C(414) & $\mathrm{H}(413)$ & 119.1(1) & $\operatorname{Rh}(2)$ & $\mathrm{C}(203)$ & $\mathrm{C}(202)$ & $72.3(5)$ \\
\hline $\operatorname{Rh}(2)$ & $C(203)$ & $\mathrm{C}(204)$ & $96.6(6)$ & $\operatorname{Rh}(2)$ & $C(203)$ & $\mathrm{H}(202)$ & $120.6(9)$ \\
\hline$C(202)$ & $C(203)$ & $\mathrm{H}(202)$ & $120.6(1)$ & $C(204)$ & $C(203)$ & $\mathrm{H}(202)$ & $120.8(1)$ \\
\hline C(207) & $\mathrm{C}(208)$ & $\mathrm{H}(207)$ & $109.0(1)$ & $\mathrm{C}(204)$ & $C(208)$ & $\mathrm{H}(207)$ & 109.7(1) \\
\hline C(207) & $\mathrm{C}(208)$ & $\mathrm{H}(208)$ & $109.7(1)$ & $C(204)$ & $\mathrm{C}(208)$ & $\mathrm{H}(208)$ & $109.4(1)$ \\
\hline $\mathrm{H}(207)$ & $\mathrm{C}(208)$ & $\mathrm{H}(208)$ & $109.5(1)$ & $\mathrm{C}(306)$ & $\mathrm{C}(301)$ & $\mathrm{H}(301)$ & $111.7(1)$ \\
\hline$C(302)$ & $\mathrm{C}(301)$ & $\mathrm{H}(301)$ & 111.4(1) & $C(307)$ & $\mathrm{C}(301)$ & $\mathrm{H}(301)$ & 111.2(1) \\
\hline C(403) & $\mathrm{C}(404)$ & $\mathrm{H}(403)$ & $111.5(9)$ & $\mathrm{C}(405)$ & C(404) & $\mathrm{H}(403)$ & $111.2(9)$ \\
\hline C(408) & C(404) & $\mathrm{H}(403)$ & $111.5(9)$ & $\mathrm{C}(401)$ & C(407) & $\mathrm{H}(405)$ & $109.0(9)$ \\
\hline C(408) & $\mathrm{C}(407)$ & $\mathrm{H}(405)$ & 109.1(9) & $\mathrm{C}(401)$ & $\mathrm{C}(407)$ & $\mathrm{H}(406)$ & 109.5(9) \\
\hline C(408) & $\mathrm{C}(407)$ & $\mathrm{H}(406)$ & $109.5(1)$ & $\mathrm{H}(405)$ & $\mathrm{C}(407)$ & $\mathrm{H}(406)$ & $109.5(1)$ \\
\hline $\operatorname{Rh}(3)$ & $C(302)$ & C(303) & $69.1(5)$ & $\operatorname{Rh}(3)$ & $\mathrm{C}(302)$ & $\mathrm{C}(309)$ & $114.6(6)$ \\
\hline $\operatorname{Rh}(3)$ & $\mathrm{C}(302)$ & $\mathrm{C}(301)$ & $94.8(5)$ & $C(314)$ & $\mathrm{C}(313)$ & $\mathrm{H}(312)$ & 119.9(1) \\
\hline$C(312)$ & $\mathrm{C}(313)$ & $\mathrm{H}(312)$ & $119.4(1)$ & $\mathrm{C}(515)$ & $\mathrm{C}(516)$ & $\mathrm{H}(514)$ & $119.8(1)$ \\
\hline $\mathrm{C}(517)$ & $C(516)$ & $\mathrm{H}(514)$ & 119.7(1) & $\operatorname{Rh}(2)$ & $C(202)$ & $C(203)$ & $68.8(6)$ \\
\hline $\operatorname{Rh}(2)$ & $C(202)$ & C(209) & $118.5(6)$ & $\operatorname{Rh}(2)$ & $C(202)$ & $C(201)$ & $93.9(5)$ \\
\hline $\mathrm{Rh}(2)$ & $C(205)$ & $C(206)$ & $69.8(5)$ & $\operatorname{Rh}(2)$ & $C(205)$ & $C(215)$ & $113.7(6)$ \\
\hline $\mathrm{Rh}(2)$ & $C(205)$ & $C(204)$ & $94.7(5)$ & $\mathrm{C}(115)$ & $\mathrm{C}(120)$ & $\mathrm{H}(118)$ & $118.9(1)$ \\
\hline $\mathrm{C}(119)$ & $\mathrm{C}(120)$ & $\mathrm{H}(118)$ & $119.6(1)$ & $\mathrm{C}(419)$ & $\mathrm{C}(420)$ & $\mathrm{H}(418)$ & $119.8(1)$ \\
\hline C(415) & $\mathrm{C}(420)$ & $\mathrm{H}(418)$ & 119.2(1) & $\mathrm{C}(613)$ & $\mathrm{C}(614)$ & $\mathrm{H}(613)$ & $119.8(1)$ \\
\hline $\mathrm{C}(609)$ & $\mathrm{C}(614)$ & $\mathrm{H}(613)$ & 119.1(1) & $\mathrm{C}(315)$ & $\mathrm{C}(320)$ & $\mathrm{H}(318)$ & $119.5(1)$ \\
\hline C(319) & $\mathrm{C}(320)$ & $\mathrm{H}(318)$ & $120.2(1)$ & $\mathrm{C}(318)$ & $\mathrm{C}(319)$ & $\mathrm{H}(317)$ & $119.5(1)$ \\
\hline C(320) & $\mathrm{C}(319)$ & $\mathrm{H}(317)$ & $119.2(1)$ & $C(203)$ & $C(204)$ & $\mathrm{H}(203)$ & $111.8(1)$ \\
\hline $\mathrm{C}(208)$ & C(204) & $\mathrm{H}(203)$ & $111.2(1)$ & $C(205)$ & $\mathrm{C}(204)$ & $\mathrm{H}(203)$ & $110.9(1)$ \\
\hline C(109) & $\mathrm{C}(110)$ & H(109) & $119.9(1)$ & $\mathrm{C}(111)$ & $\mathrm{C}(110)$ & $\mathrm{H}(109)$ & $120.2(1)$ \\
\hline $\mathrm{C}(101)$ & C(107) & $\mathrm{H}(105)$ & $108.8(1)$ & $\mathrm{C}(108)$ & $\mathrm{C}(107)$ & $\mathrm{H}(105)$ & 109.4(1) \\
\hline $\mathrm{C}(101)$ & C(107) & $\mathrm{H}(106)$ & $109.2(1)$ & $\mathrm{C}(108)$ & $\mathrm{C}(107)$ & $\mathrm{H}(106)$ & $109.3(1)$ \\
\hline $\mathrm{H}(105)$ & C(107) & $\mathrm{H}(106)$ & $109.5(1)$ & $C(613)$ & $\mathrm{C}(612)$ & $\mathrm{H}(611)$ & $120.4(1)$ \\
\hline C(611) & $\mathrm{C}(612)$ & $\mathrm{H}(611)$ & $120.1(1)$ & C(616) & $\mathrm{C}(617)$ & $\mathrm{H}(615)$ & 119.2(1) \\
\hline C(618) & C(617) & $\mathrm{H}(615)$ & $119.5(1)$ & $\mathrm{C}(111)$ & $\mathrm{C}(112)$ & $\mathrm{H}(111)$ & $120.7(1)$ \\
\hline C(113) & $C(112)$ & $\mathrm{H}(111)$ & $120.7(1)$ & $\mathrm{C}(520)$ & $\mathrm{C}(519)$ & $\mathrm{H}(517)$ & $119.6(1)$ \\
\hline $\mathrm{C}(518)$ & $\mathrm{C}(519)$ & $\mathrm{H}(517)$ & $119.6(1)$ & $C(316)$ & $\mathrm{C}(317)$ & $\mathrm{H}(315)$ & $119.6(1)$ \\
\hline C(318) & $\mathrm{C}(317)$ & $\mathrm{H}(315)$ & $119.7(1)$ & $\mathrm{C}(601)$ & $\mathrm{C}(607)$ & $\mathrm{H}(605)$ & $109.5(1)$ \\
\hline C(608) & C(607) & $\mathrm{H}(605)$ & $109.6(1)$ & $\mathrm{C}(601)$ & $\mathrm{C}(607)$ & $\mathrm{H}(606)$ & 109.4(1) \\
\hline C(608) & C(607) & $\mathrm{H}(606)$ & 109.4(1) & $\mathrm{H}(605)$ & $\mathrm{C}(607)$ & $\mathrm{H}(606)$ & $109.5(1)$ \\
\hline $\mathrm{C}(509)$ & $C(514)$ & $\mathrm{H}(513)$ & 119.3(1) & $C(513)$ & $C(514)$ & $\mathrm{H}(513)$ & $120.1(1)$ \\
\hline $\mathrm{C}(507)$ & $\mathrm{C}(508)$ & $\mathrm{H}(507)$ & $109.0(1)$ & $C(504)$ & $C(508)$ & $\mathrm{H}(507)$ & 109.1(1) \\
\hline $\mathrm{C}(507)$ & $\mathrm{C}(508)$ & $\mathrm{H}(508)$ & $109.2(1)$ & $C(504)$ & $C(508)$ & $\mathrm{H}(508)$ & 109.2(1) \\
\hline $\mathrm{H}(507)$ & $\mathrm{C}(508)$ & $\mathrm{H}(508)$ & $109.5(1)$ & $C(218)$ & $\mathrm{C}(219)$ & $\mathrm{H}(217)$ & $120.7(2)$ \\
\hline$C(220)$ & $\mathrm{C}(219)$ & $\mathrm{H}(217)$ & 119.3(1) & $\operatorname{Rh}(5)$ & $C(502)$ & $C(503)$ & $69.2(5)$ \\
\hline $\operatorname{Rh}(5)$ & $\mathrm{C}(502)$ & $\mathrm{C}(509)$ & $117.5(6)$ & $\operatorname{Rh}(5)$ & $\mathrm{C}(502)$ & $\mathrm{C}(501)$ & $93.9(5)$ \\
\hline
\end{tabular}




$\begin{array}{llllllll}\mathrm{C}(308) & \mathrm{C}(307) & \mathrm{H}(306) & 108.7(1) & \mathrm{C}(301) & \mathrm{C}(307) & \mathrm{H}(306) & 109.1(1) \\ \mathrm{C}(308) & \mathrm{C}(307) & \mathrm{H}(305) & 108.8(1) & \mathrm{C}(301) & \mathrm{C}(307) & \mathrm{H}(305) & 109.3(1) \\ \mathrm{H}(306) & \mathrm{C}(307) & \mathrm{H}(305) & 109.5(1) & \mathrm{C}(207) & \mathrm{C}(201) & \mathrm{H}(201) & 112.1(1) \\ \mathrm{C}(206) & \mathrm{C}(201) & \mathrm{H}(201) & 111.8(1) & \mathrm{C}(202) & \mathrm{C}(201) & \mathrm{H}(201) & 111.2(1) \\ \mathrm{C}(118) & \mathrm{C}(119) & \mathrm{H}(117) & 120.2(1) & \mathrm{C}(120) & \mathrm{C}(119) & \mathrm{H}(117) & 119.8(1) \\ \mathrm{Rh}(5) & \mathrm{C}(506) & \mathrm{C}(505) & 72.6(5) & \mathrm{Rh}(5) & \mathrm{C}(506) & \mathrm{C}(501) & 95.8(5) \\ \mathrm{Rh}(5) & \mathrm{C}(506) & \mathrm{H}(504) & 120.4(8) & \mathrm{C}(505) & \mathrm{C}(506) & \mathrm{H}(504) & 120.4(1) \\ \mathrm{C}(501) & \mathrm{C}(506) & \mathrm{H}(504) & 120.9(1) & \mathrm{C}(213) & \mathrm{C}(212) & \mathrm{H}(211) & 120.2(1) \\ \mathrm{C}(211) & \mathrm{C}(212) & \mathrm{H}(211) & 120.2(1) & \mathrm{C}(511) & \mathrm{C}(512) & \mathrm{H}(511) & 120.2(1) \\ \mathrm{C}(513) & \mathrm{C}(512) & \mathrm{H}(511) & 121.0(1) & \mathrm{C}(213) & \mathrm{C}(214) & \mathrm{H}(213) & 119.5(1) \\ \mathrm{C}(209) & \mathrm{C}(214) & \mathrm{H}(213) & 118.6(1) & \mathrm{C}(604) & \mathrm{C}(608) & \mathrm{H}(608) & 109.8(1) \\ \mathrm{C}(607) & \mathrm{C}(608) & \mathrm{H}(608) & 109.6(1) & \mathrm{C}(604) & \mathrm{C}(608) & \mathrm{H}(607) & 109.3(1) \\ \mathrm{C}(607) & \mathrm{C}(608) & \mathrm{H}(607) & 109.4(1) & \mathrm{H}(608) & \mathrm{C}(608) & \mathrm{H}(607) & 109.5(1) \\ \mathrm{C}(517) & \mathrm{C}(518) & \mathrm{H}(516) & 121.0(1) & \mathrm{C}(519) & \mathrm{C}(518) & \mathrm{H}(516) & 121.5(1) \\ \mathrm{C}(116) & \mathrm{C}(117) & \mathrm{H}(115) & 120.1(1) & \mathrm{C}(118) & \mathrm{C}(117) & \mathrm{H}(115) & 120.4(1) \\ \mathrm{C}(507) & \mathrm{C}(501) & \mathrm{H}(501) & 112.0(1) & \mathrm{C}(502) & \mathrm{C}(501) & \mathrm{H}(501) & 111.5(1) \\ \mathrm{C}(506) & \mathrm{C}(501) & \mathrm{H}(501) & 111.8(1) & \mathrm{C}(503) & \mathrm{C}(504) & \mathrm{H}(503) & 111.9(1) \\ \mathrm{C}(505) & \mathrm{C}(504) & \mathrm{H}(503) & 111.3(1) & \mathrm{C}(508) & \mathrm{C}(504) & \mathrm{H}(503) & 112.1(1) \\ \mathrm{C}(215) & \mathrm{C}(220) & \mathrm{H}(218) & 118.9(1) & \mathrm{C}(219) & \mathrm{C}(220) & \mathrm{H}(218) & 120.3(1) \\ \mathrm{C}(514) & \mathrm{C}(513) & \mathrm{H}(512) & 119.7(1) & \mathrm{C}(512) & \mathrm{C}(513) & \mathrm{H}(512) & 119.2(1)\end{array}$

Table S10. Torsion Angles $\left({ }^{\circ}\right)$

\begin{tabular}{|c|c|c|c|c|c|c|c|c|c|}
\hline atom & atom & & atom & angle & atom & atom & atom & atom & angle \\
\hline $\mathrm{Cl}(6)$ & $\operatorname{Rh}(5)$ & $\operatorname{Rh}(6)$ & $\mathrm{Cl}(6)$ & $0.0(2)$ & $\mathrm{Cl}(6)$ & $\operatorname{Rh}(5)$ & $\operatorname{Rh}(6)$ & $\mathrm{Cl}(5)$ & $115.7(2)$ \\
\hline $\mathrm{Cl}(6)$ & $\mathrm{Rh}(5)$ & $\operatorname{Rh}(6)$ & $\mathrm{C}(605)$ & $-89.1(5)$ & $\mathrm{Cl}(6)$ & $\operatorname{Rh}(5)$ & $\operatorname{Rh}(6)$ & $\mathrm{C}(602)$ & $164.2(5)$ \\
\hline $\mathrm{Cl}(6)$ & $\mathrm{Rh}(5)$ & $\operatorname{Rh}(6)$ & $\mathrm{C}(603)$ & $-162.7(5)$ & $\mathrm{Cl}(6)$ & $\operatorname{Rh}(5)$ & $\operatorname{Rh}(6)$ & $C(606)$ & $-56.1(5)$ \\
\hline $\mathrm{Cl}(5)$ & $\mathrm{Rh}(5)$ & $\operatorname{Rh}(6)$ & $\mathrm{Cl}(6)$ & $-115.7(2)$ & $\mathrm{Cl}(5)$ & $\operatorname{Rh}(5)$ & $\operatorname{Rh}(6)$ & $\mathrm{Cl}(5)$ & $0.0(2)$ \\
\hline $\mathrm{Cl}(5)$ & $\mathrm{Rh}(5)$ & $\operatorname{Rh}(6)$ & $\mathrm{C}(605)$ & $155.2(5)$ & $\mathrm{Cl}(5)$ & $\operatorname{Rh}(5)$ & $\operatorname{Rh}(6)$ & $\mathrm{C}(602)$ & $48.5(6)$ \\
\hline $\mathrm{Cl}(5)$ & $\operatorname{Rh}(5)$ & $\mathrm{Rh}(6)$ & $\mathrm{C}(603)$ & $81.6(5)$ & $\mathrm{Cl}(5)$ & $\operatorname{Rh}(5)$ & $\operatorname{Rh}(6)$ & $C(606)$ & $-171.8(5)$ \\
\hline$C(503)$ & $\operatorname{Rh}(5)$ & $\mathrm{Rh}(6)$ & $\mathrm{Cl}(6)$ & $80.5(1)$ & $C(503)$ & $\operatorname{Rh}(5)$ & $\operatorname{Rh}(6)$ & $\mathrm{Cl}(5)$ & $-163.8(1)$ \\
\hline$C(503)$ & $\operatorname{Rh}(5)$ & $\operatorname{Rh}(6)$ & $\mathrm{C}(605)$ & $-8.6(1)$ & $\mathrm{C}(503)$ & $\operatorname{Rh}(5)$ & $\operatorname{Rh}(6)$ & $\mathrm{C}(602)$ & $-115.3(1)$ \\
\hline $\mathrm{C}(503)$ & $\operatorname{Rh}(5)$ & $\mathrm{Rh}(6)$ & $\mathrm{C}(603)$ & $-82.2(1)$ & $C(503)$ & $\operatorname{Rh}(5)$ & $\operatorname{Rh}(6)$ & $C(606)$ & $24.4(2)$ \\
\hline $\mathrm{C}(505)$ & $\operatorname{Rh}(5)$ & $\mathrm{Rh}(6)$ & $\mathrm{Cl}(6)$ & $154.8(1)$ & $C(505)$ & $\operatorname{Rh}(5)$ & $\operatorname{Rh}(6)$ & $\mathrm{Cl}(5)$ & $-89.5(1)$ \\
\hline$C(505)$ & $\operatorname{Rh}(5)$ & $\mathrm{Rh}(6)$ & $\mathrm{C}(605)$ & $65.7(1)$ & $C(505)$ & $\operatorname{Rh}(5)$ & $\operatorname{Rh}(6)$ & $C(602)$ & $-41.0(2)$ \\
\hline$C(505)$ & $\operatorname{Rh}(5)$ & $\mathrm{Rh}(6)$ & $\mathrm{C}(603)$ & $-8.0(1)$ & $\mathrm{C}(505)$ & $\operatorname{Rh}(5)$ & $\operatorname{Rh}(6)$ & $C(606)$ & $98.7(1)$ \\
\hline$C(502)$ & $\operatorname{Rh}(5)$ & $\mathrm{Rh}(6)$ & $\mathrm{Cl}(6)$ & $44.0(2)$ & $\mathrm{C}(502)$ & $\operatorname{Rh}(5)$ & $\operatorname{Rh}(6)$ & $\mathrm{Cl}(5)$ & $159.7(2)$ \\
\hline$C(502)$ & $\operatorname{Rh}(5)$ & $\mathrm{Rh}(6)$ & $\mathrm{C}(605)$ & $-45.1(2)$ & $\mathrm{C}(502)$ & $\operatorname{Rh}(5)$ & $\operatorname{Rh}(6)$ & $\mathrm{C}(602)$ & $-151.8(2)$ \\
\hline $\mathrm{C}(502)$ & $\operatorname{Rh}(5)$ & $\operatorname{Rh}(6)$ & $\mathrm{C}(603)$ & $-118.7(2)$ & $\mathrm{C}(502)$ & $\operatorname{Rh}(5)$ & $\operatorname{Rh}(6)$ & $\mathrm{C}(606)$ & $-12.1(3)$ \\
\hline$C(506)$ & $\operatorname{Rh}(5)$ & $\operatorname{Rh}(6)$ & $\mathrm{Cl}(6)$ & $-169.5(2)$ & $\mathrm{C}(506)$ & $\operatorname{Rh}(5)$ & $\operatorname{Rh}(6)$ & $\mathrm{Cl}(5)$ & $-53.8(2)$ \\
\hline$C(506)$ & $\operatorname{Rh}(5)$ & $\operatorname{Rh}(6)$ & $C(605)$ & $101.5(2)$ & $C(506)$ & $\operatorname{Rh}(5)$ & $\operatorname{Rh}(6)$ & $\mathrm{C}(602)$ & $-5.3(3)$ \\
\hline$C(506)$ & $\operatorname{Rh}(5)$ & $\operatorname{Rh}(6)$ & C(603) & $27.8(2)$ & $C(506)$ & $\operatorname{Rh}(5)$ & $\operatorname{Rh}(6)$ & C(606) & $134.4(2)$ \\
\hline $\operatorname{Rh}(6)$ & $\operatorname{Rh}(5)$ & $\mathrm{Cl}(6)$ & $\operatorname{Rh}(6)$ & $0.00(7)$ & $\mathrm{Cl}(5)$ & $\operatorname{Rh}(5)$ & $\mathrm{Cl}(6)$ & $\operatorname{Rh}(6)$ & $45.9(2)$ \\
\hline$C(503)$ & $\operatorname{Rh}(5)$ & $\mathrm{Cl}(6)$ & $\operatorname{Rh}(6)$ & $-117.8(9)$ & $C(505)$ & $\operatorname{Rh}(5)$ & $\mathrm{Cl}(6)$ & $\operatorname{Rh}(6)$ & $-64.6(2)$ \\
\hline$C(502)$ & $\operatorname{Rh}(5)$ & $\mathrm{Cl}(6)$ & $\operatorname{Rh}(6)$ & $-157.8(9)$ & $C(506)$ & $\operatorname{Rh}(5)$ & $\mathrm{Cl}(6)$ & $\operatorname{Rh}(6)$ & $155.8(4)$ \\
\hline $\operatorname{Rh}(6)$ & $\operatorname{Rh}(5)$ & $\mathrm{Cl}(5)$ & $\operatorname{Rh}(6)$ & $0.00(7)$ & $\mathrm{Cl}(6)$ & $\operatorname{Rh}(5)$ & $\mathrm{Cl}(5)$ & $\operatorname{Rh}(6)$ & $-46.1(2)$ \\
\hline$C(503)$ & $\operatorname{Rh}(5)$ & $\mathrm{Cl}(5)$ & $\operatorname{Rh}(6)$ & $59.3(4)$ & $C(505)$ & $\operatorname{Rh}(5)$ & $\mathrm{Cl}(5)$ & $\operatorname{Rh}(6)$ & $109.2(9)$ \\
\hline $\mathrm{C}(502)$ & $\operatorname{Rh}(5)$ & $\mathrm{Cl}(5)$ & $\operatorname{Rh}(6)$ & $-153.3(3)$ & $\mathrm{C}(506)$ & $\operatorname{Rh}(5)$ & $\mathrm{Cl}(5)$ & $\operatorname{Rh}(6)$ & $149.3(9)$ \\
\hline $\operatorname{Rh}(6)$ & $\operatorname{Rh}(5)$ & $\mathrm{C}(503)$ & $\mathrm{C}(502)$ & $-150.0(6)$ & $\mathrm{Rh}(6)$ & $\operatorname{Rh}(5)$ & $C(503)$ & $C(504)$ & $97.3(9)$ \\
\hline $\mathrm{Cl}(6)$ & $\operatorname{Rh}(5)$ & $\mathrm{C}(503)$ & $C(502)$ & $-97.2(8)$ & $\mathrm{Cl}(6)$ & $\operatorname{Rh}(5)$ & $\mathrm{C}(503)$ & $C(504)$ & $150.1(8)$ \\
\hline $\mathrm{Cl}(5)$ & $\operatorname{Rh}(5)$ & $\mathrm{C}(503)$ & $\mathrm{C}(502)$ & $159.5(1)$ & $\mathrm{Cl}(5)$ & $\operatorname{Rh}(5)$ & $C(503)$ & $C(504)$ & $46.8(2)$ \\
\hline
\end{tabular}




\begin{tabular}{|c|c|c|c|c|c|c|c|c|c|}
\hline$C(505)$ & $\operatorname{Rh}(5)$ & $C(503)$ & $C(502)$ & 105.1(1) & $C(505)$ & $\operatorname{Rh}(5)$ & $C(503)$ & $C(504)$ & $-7.5(9)$ \\
\hline$C(502)$ & $\operatorname{Rh}(5)$ & $\mathrm{C}(503)$ & $\mathrm{C}(502)$ & $0.0(7)$ & $C(502)$ & $\operatorname{Rh}(5)$ & $\mathrm{C}(503)$ & $C(504)$ & $-112.7(1)$ \\
\hline$C(506)$ & $\operatorname{Rh}(5)$ & $\mathrm{C}(503)$ & $\mathrm{C}(502)$ & $66.5(1)$ & $C(506)$ & $\operatorname{Rh}(5)$ & $C(503)$ & $C(504)$ & $-46.2(1)$ \\
\hline $\operatorname{Rh}(6)$ & $\operatorname{Rh}(5)$ & $\mathrm{C}(505)$ & $\mathrm{C}(515)$ & $25.4(1)$ & $\operatorname{Rh}(6)$ & $\operatorname{Rh}(5)$ & $C(505)$ & $C(506)$ & $144.9(7)$ \\
\hline $\operatorname{Rh}(6)$ & $\operatorname{Rh}(5)$ & $C(505)$ & C(504) & $-103.8(9)$ & $\mathrm{Cl}(6)$ & $\operatorname{Rh}(5)$ & $C(505)$ & $C(515)$ & $77.0(2)$ \\
\hline $\mathrm{Cl}(6)$ & $\operatorname{Rh}(5)$ & $C(505)$ & C(506) & $-163.5(8)$ & $\mathrm{Cl}(6)$ & $\operatorname{Rh}(5)$ & $C(505)$ & $C(504)$ & $-52.2(2)$ \\
\hline $\mathrm{Cl}(5)$ & $\operatorname{Rh}(5)$ & $C(505)$ & $\mathrm{C}(515)$ & $-29.4(1)$ & $\mathrm{Cl}(5)$ & $\operatorname{Rh}(5)$ & $C(505)$ & $C(506)$ & $90.2(8)$ \\
\hline $\mathrm{Cl}(5)$ & $\operatorname{Rh}(5)$ & $C(505)$ & C(504) & $-158.6(8)$ & $C(503)$ & $\operatorname{Rh}(5)$ & $C(505)$ & $C(515)$ & 136.7(1) \\
\hline$C(503)$ & $\operatorname{Rh}(5)$ & $C(505)$ & $C(506)$ & $-103.7(1)$ & $C(503)$ & $\operatorname{Rh}(5)$ & $C(505)$ & $C(504)$ & $7.5(9)$ \\
\hline$C(502)$ & $\operatorname{Rh}(5)$ & $C(505)$ & $C(515)$ & 175.1(1) & $C(502)$ & $\operatorname{Rh}(5)$ & $C(505)$ & $C(506)$ & $-65.4(1)$ \\
\hline$C(502)$ & $\operatorname{Rh}(5)$ & $C(505)$ & C(504) & $45.9(1)$ & $C(506)$ & $\operatorname{Rh}(5)$ & $C(505)$ & $C(515)$ & $-119.5(2)$ \\
\hline$C(506)$ & $\operatorname{Rh}(5)$ & $\mathrm{C}(505)$ & $C(506)$ & $0.0(7)$ & $C(506)$ & $\operatorname{Rh}(5)$ & $C(505)$ & $C(504)$ & 111.3(1) \\
\hline $\operatorname{Rh}(6)$ & $\operatorname{Rh}(5)$ & $C(502)$ & $C(503)$ & $56.1(1)$ & $\operatorname{Rh}(6)$ & $\operatorname{Rh}(5)$ & $C(502)$ & $\mathrm{C}(509)$ & $-62.1(2)$ \\
\hline $\operatorname{Rh}(6)$ & $\operatorname{Rh}(5)$ & $C(502)$ & $\mathrm{C}(501)$ & $168.0(5)$ & $\mathrm{Cl}(6)$ & $\operatorname{Rh}(5)$ & $\mathrm{C}(502)$ & $C(503)$ & $90.6(8)$ \\
\hline $\mathrm{Cl}(6)$ & $\operatorname{Rh}(5)$ & $\mathrm{C}(502)$ & $\mathrm{C}(509)$ & $-27.7(1)$ & $\mathrm{Cl}(6)$ & $\operatorname{Rh}(5)$ & $C(502)$ & $C(501)$ & $-157.6(8)$ \\
\hline $\mathrm{Cl}(5)$ & $\operatorname{Rh}(5)$ & $\mathrm{C}(502)$ & $C(503)$ & $-165.9(9)$ & $\mathrm{Cl}(5)$ & $\operatorname{Rh}(5)$ & $C(502)$ & $\mathrm{C}(509)$ & $75.9(2)$ \\
\hline $\mathrm{Cl}(5)$ & $\operatorname{Rh}(5)$ & $C(502)$ & $\mathrm{C}(501)$ & $-54.0(2)$ & $C(503)$ & $\operatorname{Rh}(5)$ & $C(502)$ & $C(503)$ & $0.0(7)$ \\
\hline$C(503)$ & $\operatorname{Rh}(5)$ & $C(502)$ & C(509) & $-118.3(2)$ & $C(503)$ & $\operatorname{Rh}(5)$ & $C(502)$ & $C(501)$ & $111.8(1)$ \\
\hline$C(505)$ & $\operatorname{Rh}(5)$ & $C(502)$ & $C(503)$ & $-63.4(1)$ & $C(505)$ & $\operatorname{Rh}(5)$ & $C(502)$ & $\mathrm{C}(509)$ & $178.3(1)$ \\
\hline$C(505)$ & $\operatorname{Rh}(5)$ & $C(502)$ & $C(501)$ & 48.4(1) & $C(506)$ & $\operatorname{Rh}(5)$ & $\mathrm{C}(502)$ & $C(503)$ & $-102.0(1)$ \\
\hline$C(506)$ & $\operatorname{Rh}(5)$ & $C(502)$ & $\mathrm{C}(509)$ & $139.7(1)$ & $C(506)$ & $\operatorname{Rh}(5)$ & $C(502)$ & $C(501)$ & $9.8(9)$ \\
\hline $\operatorname{Rh}(6)$ & $\operatorname{Rh}(5)$ & $C(506)$ & $C(505)$ & $-57.9(1)$ & $\operatorname{Rh}(6)$ & $\operatorname{Rh}(5)$ & $C(506)$ & $C(501)$ & $-171.3(5)$ \\
\hline $\mathrm{Cl}(6)$ & $\operatorname{Rh}(5)$ & $C(506)$ & $C(505)$ & $153.7(2)$ & $\mathrm{Cl}(6)$ & $\operatorname{Rh}(5)$ & $C(506)$ & $C(501)$ & 40.3(2) \\
\hline $\mathrm{Cl}(5)$ & $\operatorname{Rh}(5)$ & $C(506)$ & $C(505)$ & $-98.6(8)$ & $\mathrm{Cl}(5)$ & $\operatorname{Rh}(5)$ & $C(506)$ & $C(501)$ & $148.0(8)$ \\
\hline$C(503)$ & $\operatorname{Rh}(5)$ & $C(506)$ & $C(505)$ & $64.5(9)$ & $C(503)$ & $\operatorname{Rh}(5)$ & $C(506)$ & $C(501)$ & $-48.9(1)$ \\
\hline$C(505)$ & $\operatorname{Rh}(5)$ & $C(506)$ & $C(505)$ & $0.0(7)$ & $C(505)$ & $\operatorname{Rh}(5)$ & $C(506)$ & $\mathrm{C}(501)$ & $-113.4(1)$ \\
\hline$C(502)$ & $\operatorname{Rh}(5)$ & $\mathrm{C}(506)$ & $C(505)$ & $103.5(1)$ & $C(502)$ & $\operatorname{Rh}(5)$ & $C(506)$ & $C(501)$ & $-9.9(9)$ \\
\hline $\mathrm{Cl}(2)$ & $\operatorname{Rh}(2)$ & $\operatorname{Rh}(1)$ & $\mathrm{Cl}(2)$ & $0.0(2)$ & $\mathrm{Cl}(2)$ & $\operatorname{Rh}(2)$ & $\operatorname{Rh}(1)$ & $\mathrm{Cl}(1)$ & $-111.8(2)$ \\
\hline $\mathrm{Cl}(2)$ & $\operatorname{Rh}(2)$ & $\operatorname{Rh}(1)$ & $C(102)$ & $60.6(6)$ & $\mathrm{Cl}(2)$ & $\operatorname{Rh}(2)$ & $\operatorname{Rh}(1)$ & $C(103)$ & 84.7(5) \\
\hline $\mathrm{Cl}(2)$ & $\operatorname{Rh}(2)$ & $\operatorname{Rh}(1)$ & C(106) & $-177.8(6)$ & $\mathrm{Cl}(2)$ & $\operatorname{Rh}(2)$ & $\operatorname{Rh}(1)$ & $C(105)$ & $155.8(5)$ \\
\hline $\mathrm{Cl}(1)$ & $\operatorname{Rh}(2)$ & $\operatorname{Rh}(1)$ & $\mathrm{Cl}(2)$ & $111.8(2)$ & $\mathrm{Cl}(1)$ & $\operatorname{Rh}(2)$ & $\operatorname{Rh}(1)$ & $\mathrm{Cl}(1)$ & $0.0(2)$ \\
\hline $\mathrm{Cl}(1)$ & $\operatorname{Rh}(2)$ & $\operatorname{Rh}(1)$ & $C(102)$ & $172.4(6)$ & $\mathrm{Cl}(1)$ & $\operatorname{Rh}(2)$ & $\operatorname{Rh}(1)$ & $C(103)$ & $-163.4(5)$ \\
\hline $\mathrm{Cl}(1)$ & $\operatorname{Rh}(2)$ & $\operatorname{Rh}(1)$ & C(106) & $-65.9(6)$ & $\mathrm{Cl}(1)$ & $\operatorname{Rh}(2)$ & $\operatorname{Rh}(1)$ & $C(105)$ & $-92.3(5)$ \\
\hline$C(206)$ & $\operatorname{Rh}(2)$ & $\operatorname{Rh}(1)$ & $\mathrm{Cl}(2)$ & $-166.4(1)$ & $C(206)$ & $\operatorname{Rh}(2)$ & $\operatorname{Rh}(1)$ & $\mathrm{Cl}(1)$ & $81.7(1)$ \\
\hline$C(206)$ & $\operatorname{Rh}(2)$ & $\operatorname{Rh}(1)$ & $C(102)$ & $-105.9(1)$ & $C(206)$ & $\operatorname{Rh}(2)$ & $\operatorname{Rh}(1)$ & $C(103)$ & $-81.7(1)$ \\
\hline$C(206)$ & $\operatorname{Rh}(2)$ & $\operatorname{Rh}(1)$ & C(106) & $15.8(2)$ & $C(206)$ & $\operatorname{Rh}(2)$ & $\operatorname{Rh}(1)$ & $C(105)$ & $-10.6(1)$ \\
\hline$C(203)$ & $\operatorname{Rh}(2)$ & $\operatorname{Rh}(1)$ & $\mathrm{Cl}(2)$ & $-63.7(2)$ & $C(203)$ & $\operatorname{Rh}(2)$ & $\operatorname{Rh}(1)$ & $\mathrm{Cl}(1)$ & $-175.6(2)$ \\
\hline$C(203)$ & $\operatorname{Rh}(2)$ & $\operatorname{Rh}(1)$ & $\mathrm{C}(102)$ & $-3.1(3)$ & $C(203)$ & $\operatorname{Rh}(2)$ & $\operatorname{Rh}(1)$ & $C(103)$ & $21.0(2)$ \\
\hline$C(203)$ & $\operatorname{Rh}(2)$ & $\operatorname{Rh}(1)$ & C(106) & $118.5(2)$ & $C(203)$ & $\operatorname{Rh}(2)$ & $\operatorname{Rh}(1)$ & $C(105)$ & $92.1(2)$ \\
\hline$C(202)$ & $\operatorname{Rh}(2)$ & $\operatorname{Rh}(1)$ & $\mathrm{Cl}(2)$ & $-93.8(1)$ & $C(202)$ & $\operatorname{Rh}(2)$ & $\operatorname{Rh}(1)$ & $\mathrm{Cl}(1)$ & $154.4(1)$ \\
\hline$C(202)$ & $\operatorname{Rh}(2)$ & $\operatorname{Rh}(1)$ & $C(102)$ & $-33.2(2)$ & $C(202)$ & $\operatorname{Rh}(2)$ & $\operatorname{Rh}(1)$ & $C(103)$ & $-9.1(1)$ \\
\hline$C(202)$ & $\operatorname{Rh}(2)$ & $\operatorname{Rh}(1)$ & C(106) & $88.4(1)$ & $C(202)$ & $\operatorname{Rh}(2)$ & $\operatorname{Rh}(1)$ & $C(105)$ & $62.0(1)$ \\
\hline$C(205)$ & $\operatorname{Rh}(2)$ & $\operatorname{Rh}(1)$ & $\mathrm{Cl}(2)$ & $163.2(2)$ & $C(205)$ & $\operatorname{Rh}(2)$ & $\operatorname{Rh}(1)$ & $\mathrm{Cl}(1)$ & $51.4(2)$ \\
\hline$C(205)$ & $\operatorname{Rh}(2)$ & $\operatorname{Rh}(1)$ & $\mathrm{C}(102)$ & $-136.2(2)$ & $C(205)$ & $\operatorname{Rh}(2)$ & $\operatorname{Rh}(1)$ & $C(103)$ & $-112.1(2)$ \\
\hline$C(205)$ & $\operatorname{Rh}(2)$ & $\operatorname{Rh}(1)$ & $C(106)$ & $-14.6(3)$ & $C(205)$ & $\operatorname{Rh}(2)$ & $\operatorname{Rh}(1)$ & $C(105)$ & $-41.0(2)$ \\
\hline $\operatorname{Rh}(1)$ & $\operatorname{Rh}(2)$ & $\mathrm{Cl}(2)$ & $\mathrm{Rh}(1)$ & $0.00(7)$ & $\mathrm{Cl}(1)$ & $\operatorname{Rh}(2)$ & $\mathrm{Cl}(2)$ & $\mathrm{Rh}(1)$ & $-48.1(2)$ \\
\hline$C(206)$ & $\operatorname{Rh}(2)$ & $\mathrm{Cl}(2)$ & $\operatorname{Rh}(1)$ & $45.4(3)$ & $C(203)$ & $\operatorname{Rh}(2)$ & $\mathrm{Cl}(2)$ & $\operatorname{Rh}(1)$ & $143.6(1)$ \\
\hline$C(202)$ & $\operatorname{Rh}(2)$ & $\mathrm{Cl}(2)$ & $\operatorname{Rh}(1)$ & $103.5(9)$ & $C(205)$ & $\operatorname{Rh}(2)$ & $\mathrm{Cl}(2)$ & $\operatorname{Rh}(1)$ & $-153.9(3)$ \\
\hline $\mathrm{Rh}(1)$ & $\operatorname{Rh}(2)$ & $\mathrm{Cl}(1)$ & $\operatorname{Rh}(1)$ & $0.00(7)$ & $\mathrm{Cl}(2)$ & $\operatorname{Rh}(2)$ & $\mathrm{Cl}(1)$ & $\operatorname{Rh}(1)$ & 48.5(2) \\
\hline$C(206)$ & $\operatorname{Rh}(2)$ & $\mathrm{Cl}(1)$ & $\operatorname{Rh}(1)$ & $-113.8(9)$ & $C(203)$ & $\operatorname{Rh}(2)$ & $\mathrm{Cl}(1)$ & $\operatorname{Rh}(1)$ & $167.2(5)$ \\
\hline$C(202)$ & $\operatorname{Rh}(2)$ & $\mathrm{Cl}(1)$ & $\operatorname{Rh}(1)$ & $-58.2(2)$ & $C(205)$ & $\operatorname{Rh}(2)$ & $\mathrm{Cl}(1)$ & $\operatorname{Rh}(1)$ & $-153.0(1)$ \\
\hline $\mathrm{Rh}(1)$ & $\operatorname{Rh}(2)$ & $C(206)$ & $C(205)$ & $-152.8(7)$ & $\operatorname{Rh}(1)$ & $\operatorname{Rh}(2)$ & $C(206)$ & $C(201)$ & 92.7(9) \\
\hline $\mathrm{Cl}(2)$ & $\operatorname{Rh}(2)$ & $C(206)$ & $C(205)$ & 168.7(1) & $\mathrm{Cl}(2)$ & $\operatorname{Rh}(2)$ & $C(206)$ & $C(201)$ & $54.1(2)$ \\
\hline
\end{tabular}




\begin{tabular}{|c|c|c|c|c|c|c|c|c|c|}
\hline $\mathrm{Cl}(1)$ & $\operatorname{Rh}(2)$ & $C(206)$ & $C(205)$ & $-100.2(8)$ & $\mathrm{Cl}(1)$ & $\operatorname{Rh}(2)$ & $C(206)$ & $C(201)$ & $145.3(9)$ \\
\hline$C(203)$ & $\operatorname{Rh}(2)$ & $C(206)$ & $C(205)$ & $66.9(1)$ & $C(203)$ & $\operatorname{Rh}(2)$ & C(206) & $\mathrm{C}(201)$ & $-47.6(1)$ \\
\hline$C(202)$ & $\operatorname{Rh}(2)$ & C(206) & $C(205)$ & 105.3(1) & $C(202)$ & $\operatorname{Rh}(2)$ & $C(206)$ & $C(201)$ & $-9.2(1)$ \\
\hline$C(205)$ & $\operatorname{Rh}(2)$ & $C(206)$ & $C(205)$ & $0.0(7)$ & $C(205)$ & $\operatorname{Rh}(2)$ & $C(206)$ & $C(201)$ & $-114.5(1)$ \\
\hline $\mathrm{Rh}(1)$ & $\operatorname{Rh}(2)$ & $C(203)$ & $C(202)$ & $-49.0(1)$ & $\mathrm{Rh}(1)$ & $\operatorname{Rh}(2)$ & $C(203)$ & $C(204)$ & $-161.5(7)$ \\
\hline $\mathrm{Cl}(2)$ & $\operatorname{Rh}(2)$ & $C(203)$ & $C(202)$ & $-96.5(9)$ & $\mathrm{Cl}(2)$ & $\operatorname{Rh}(2)$ & $C(203)$ & $C(204)$ & $151.0(9)$ \\
\hline $\mathrm{Cl}(1)$ & $\operatorname{Rh}(2)$ & $C(203)$ & $C(202)$ & $147.0(2)$ & $\mathrm{Cl}(1)$ & $\operatorname{Rh}(2)$ & $C(203)$ & $C(204)$ & $34.4(3)$ \\
\hline$C(206)$ & $\operatorname{Rh}(2)$ & $C(203)$ & $C(202)$ & $65.9(1)$ & $C(206)$ & $\operatorname{Rh}(2)$ & $C(203)$ & $C(204)$ & $-46.7(1)$ \\
\hline$C(202)$ & $\operatorname{Rh}(2)$ & $C(203)$ & $C(202)$ & $0.0(7)$ & $C(202)$ & $\operatorname{Rh}(2)$ & $C(203)$ & $C(204)$ & $-112.6(2)$ \\
\hline$C(205)$ & $\operatorname{Rh}(2)$ & $C(203)$ & $C(202)$ & $104.5(1)$ & $C(205)$ & $\operatorname{Rh}(2)$ & $C(203)$ & $C(204)$ & $-8.1(1)$ \\
\hline $\mathrm{Rh}(1)$ & $\operatorname{Rh}(2)$ & $C(202)$ & $C(203)$ & $149.0(7)$ & $\mathrm{Rh}(1)$ & $\operatorname{Rh}(2)$ & $C(202)$ & $\mathrm{C}(209)$ & $29.6(1)$ \\
\hline $\operatorname{Rh}(1)$ & $\operatorname{Rh}(2)$ & $\mathrm{C}(202)$ & $\mathrm{C}(201)$ & $-99.4(9)$ & $\mathrm{Cl}(2)$ & $\operatorname{Rh}(2)$ & $C(202)$ & $C(203)$ & $93.5(8)$ \\
\hline $\mathrm{Cl}(2)$ & $\operatorname{Rh}(2)$ & $C(202)$ & C(209) & $-26.0(1)$ & $\mathrm{Cl}(2)$ & $\operatorname{Rh}(2)$ & $C(202)$ & $C(201)$ & $-154.9(8)$ \\
\hline $\mathrm{Cl}(1)$ & $\operatorname{Rh}(2)$ & $C(202)$ & $C(203)$ & $-165.2(8)$ & $\mathrm{Cl}(1)$ & $\operatorname{Rh}(2)$ & $C(202)$ & $C(209)$ & 75.3(2) \\
\hline $\mathrm{Cl}(1)$ & $\operatorname{Rh}(2)$ & $\mathrm{C}(202)$ & $\mathrm{C}(201)$ & $-53.6(2)$ & $C(206)$ & $\operatorname{Rh}(2)$ & $C(202)$ & $C(203)$ & $-102.6(1)$ \\
\hline C(206) & $\operatorname{Rh}(2)$ & $C(202)$ & C(209) & $137.9(2)$ & $C(206)$ & $\operatorname{Rh}(2)$ & $C(202)$ & $C(201)$ & 9.0(9) \\
\hline$C(203)$ & $\operatorname{Rh}(2)$ & $C(202)$ & $C(203)$ & $0.0(8)$ & $C(203)$ & $\operatorname{Rh}(2)$ & $C(202)$ & $\mathrm{C}(209)$ & $-119.4(2)$ \\
\hline$C(203)$ & $\operatorname{Rh}(2)$ & $C(202)$ & $\mathrm{C}(201)$ & $111.6(1)$ & $C(205)$ & $\operatorname{Rh}(2)$ & $C(202)$ & $C(203)$ & $-65.0(1)$ \\
\hline$C(205)$ & $\operatorname{Rh}(2)$ & $C(202)$ & C(209) & $175.6(1)$ & $C(205)$ & $\operatorname{Rh}(2)$ & $C(202)$ & $C(201)$ & $46.7(1)$ \\
\hline $\operatorname{Rh}(1)$ & $\operatorname{Rh}(2)$ & $C(205)$ & $C(206)$ & 47.6(1) & $\operatorname{Rh}(1)$ & $\operatorname{Rh}(2)$ & $C(205)$ & $C(215)$ & $-72.6(2)$ \\
\hline $\operatorname{Rh}(1)$ & $\operatorname{Rh}(2)$ & $C(205)$ & C(204) & $157.3(7)$ & $\mathrm{Cl}(2)$ & $\operatorname{Rh}(2)$ & $C(205)$ & $C(206)$ & $-170.8(1)$ \\
\hline $\mathrm{Cl}(2)$ & $\operatorname{Rh}(2)$ & $C(205)$ & $C(215)$ & $69.0(2)$ & $\mathrm{Cl}(2)$ & $\operatorname{Rh}(2)$ & $C(205)$ & $C(204)$ & $-61.1(2)$ \\
\hline $\mathrm{Cl}(1)$ & $\operatorname{Rh}(2)$ & $C(205)$ & $C(206)$ & 87.1(8) & $\mathrm{Cl}(1)$ & $\operatorname{Rh}(2)$ & $C(205)$ & $C(215)$ & $-33.1(1)$ \\
\hline $\mathrm{Cl}(1)$ & $\operatorname{Rh}(2)$ & $C(205)$ & $C(204)$ & $-163.1(9)$ & $C(206)$ & $\operatorname{Rh}(2)$ & $C(205)$ & $C(206)$ & $0.0(7)$ \\
\hline$C(206)$ & $\operatorname{Rh}(2)$ & $C(205)$ & $C(215)$ & $-120.2(2)$ & $C(206)$ & $\operatorname{Rh}(2)$ & $C(205)$ & $C(204)$ & $109.8(1)$ \\
\hline$C(203)$ & $\operatorname{Rh}(2)$ & $C(205)$ & $C(206)$ & $-101.8(1)$ & $C(203)$ & $\operatorname{Rh}(2)$ & $C(205)$ & $C(215)$ & $138.0(1)$ \\
\hline$C(203)$ & $\operatorname{Rh}(2)$ & $\mathrm{C}(205)$ & $C(204)$ & 7.9(1) & $C(202)$ & $\operatorname{Rh}(2)$ & $C(205)$ & $C(206)$ & $-63.9(1)$ \\
\hline$C(202)$ & $\operatorname{Rh}(2)$ & $\mathrm{C}(205)$ & $\mathrm{C}(215)$ & 175.9(1) & $C(202)$ & $\operatorname{Rh}(2)$ & $C(205)$ & $C(204)$ & $45.9(1)$ \\
\hline $\mathrm{Cl}(3)$ & $\operatorname{Rh}(3)$ & $\operatorname{Rh}(4)$ & $\mathrm{Cl}(3)$ & $0.0(2)$ & $\mathrm{Cl}(3)$ & $\operatorname{Rh}(3)$ & $\operatorname{Rh}(4)$ & $\mathrm{Cl}(4)$ & $119.1(2)$ \\
\hline $\mathrm{Cl}(3)$ & $\operatorname{Rh}(3)$ & $\operatorname{Rh}(4)$ & $C(403)$ & $-49.5(5)$ & $\mathrm{Cl}(3)$ & $\operatorname{Rh}(3)$ & $\operatorname{Rh}(4)$ & $C(405)$ & $163.5(5)$ \\
\hline $\mathrm{Cl}(3)$ & $\operatorname{Rh}(3)$ & $\operatorname{Rh}(4)$ & $\mathrm{C}(406)$ & $-159.8(5)$ & $\mathrm{Cl}(3)$ & $\operatorname{Rh}(3)$ & $\operatorname{Rh}(4)$ & $C(402)$ & $-84.7(5)$ \\
\hline $\mathrm{Cl}(4)$ & $\operatorname{Rh}(3)$ & $\operatorname{Rh}(4)$ & $\mathrm{Cl}(3)$ & $-119.1(2)$ & $\mathrm{Cl}(4)$ & $\operatorname{Rh}(3)$ & $\operatorname{Rh}(4)$ & $\mathrm{Cl}(4)$ & $0.0(2)$ \\
\hline $\mathrm{Cl}(4)$ & $\operatorname{Rh}(3)$ & $\operatorname{Rh}(4)$ & $C(403)$ & $-168.6(5)$ & $\mathrm{Cl}(4)$ & $\operatorname{Rh}(3)$ & $\operatorname{Rh}(4)$ & $C(405)$ & $44.4(5)$ \\
\hline $\mathrm{Cl}(4)$ & $\operatorname{Rh}(3)$ & $\operatorname{Rh}(4)$ & $C(406)$ & $81.1(5)$ & $\mathrm{Cl}(4)$ & $\operatorname{Rh}(3)$ & $\operatorname{Rh}(4)$ & $C(402)$ & $156.2(5)$ \\
\hline$C(303)$ & $\operatorname{Rh}(3)$ & $\operatorname{Rh}(4)$ & $\mathrm{Cl}(3)$ & $-174.3(2)$ & $C(303)$ & $\operatorname{Rh}(3)$ & $\operatorname{Rh}(4)$ & $\mathrm{Cl}(4)$ & $-55.2(1)$ \\
\hline$C(303)$ & $\operatorname{Rh}(3)$ & $\operatorname{Rh}(4)$ & $C(403)$ & $136.2(1)$ & $C(303)$ & $\operatorname{Rh}(3)$ & $\operatorname{Rh}(4)$ & $C(405)$ & $-10.8(2)$ \\
\hline$C(303)$ & $\operatorname{Rh}(3)$ & $\operatorname{Rh}(4)$ & $C(406)$ & $25.9(2)$ & $C(303)$ & $\operatorname{Rh}(3)$ & $\operatorname{Rh}(4)$ & $C(402)$ & $100.9(2)$ \\
\hline C(306) & $\operatorname{Rh}(3)$ & $\operatorname{Rh}(4)$ & $\mathrm{Cl}(3)$ & $74.7(1)$ & $C(306)$ & $\operatorname{Rh}(3)$ & $\operatorname{Rh}(4)$ & $\mathrm{Cl}(4)$ & $-166.3(1)$ \\
\hline$C(306)$ & $\operatorname{Rh}(3)$ & $\operatorname{Rh}(4)$ & $C(403)$ & $25.1(2)$ & $C(306)$ & $\operatorname{Rh}(3)$ & $\operatorname{Rh}(4)$ & $C(405)$ & $-121.8(1)$ \\
\hline$C(306)$ & $\operatorname{Rh}(3)$ & $\operatorname{Rh}(4)$ & C(406) & $-85.1(1)$ & $C(306)$ & $\operatorname{Rh}(3)$ & $\operatorname{Rh}(4)$ & $C(402)$ & $-10.1(1)$ \\
\hline$C(305)$ & $\operatorname{Rh}(3)$ & $\operatorname{Rh}(4)$ & $\mathrm{Cl}(3)$ & $37.5(2)$ & $C(305)$ & $\operatorname{Rh}(3)$ & $\operatorname{Rh}(4)$ & $\mathrm{Cl}(4)$ & $156.6(3)$ \\
\hline$C(305)$ & $\operatorname{Rh}(3)$ & $\operatorname{Rh}(4)$ & $C(403)$ & $-12.1(3)$ & $C(305)$ & $\operatorname{Rh}(3)$ & $\operatorname{Rh}(4)$ & $C(405)$ & $-159.0(2)$ \\
\hline$C(305)$ & $\mathrm{Rh}(3)$ & $\operatorname{Rh}(4)$ & $C(406)$ & $-122.3(2)$ & $C(305)$ & $\operatorname{Rh}(3)$ & $\operatorname{Rh}(4)$ & $C(402)$ & $-47.3(2)$ \\
\hline$C(302)$ & $\operatorname{Rh}(3)$ & $\operatorname{Rh}(4)$ & $\mathrm{Cl}(3)$ & 149.8(1) & $C(302)$ & $\operatorname{Rh}(3)$ & $\operatorname{Rh}(4)$ & $\mathrm{Cl}(4)$ & $-91.1(1)$ \\
\hline$C(302)$ & $\operatorname{Rh}(3)$ & $\operatorname{Rh}(4)$ & $C(403)$ & $100.3(1)$ & $C(302)$ & $\operatorname{Rh}(3)$ & $\operatorname{Rh}(4)$ & $C(405)$ & $-46.7(2)$ \\
\hline$C(302)$ & $\operatorname{Rh}(3)$ & $\operatorname{Rh}(4)$ & C(406) & $-10.0(1)$ & $C(302)$ & $\operatorname{Rh}(3)$ & $\operatorname{Rh}(4)$ & $C(402)$ & $65.0(1)$ \\
\hline $\operatorname{Rh}(4)$ & $\operatorname{Rh}(3)$ & $\mathrm{Cl}(3)$ & $\mathrm{Rh}(4)$ & $0.00(7)$ & $\mathrm{Cl}(4)$ & $\operatorname{Rh}(3)$ & $\mathrm{Cl}(3)$ & $\mathrm{Rh}(4)$ & 43.1(2) \\
\hline$C(303)$ & $\operatorname{Rh}(3)$ & $\mathrm{Cl}(3)$ & $\operatorname{Rh}(4)$ & $159.9(5)$ & $C(306)$ & $\operatorname{Rh}(3)$ & $\mathrm{Cl}(3)$ & $\operatorname{Rh}(4)$ & $-123.0(8)$ \\
\hline$C(305)$ & $\operatorname{Rh}(3)$ & $\mathrm{Cl}(3)$ & $\operatorname{Rh}(4)$ & $-162.2(9)$ & $C(302)$ & $\operatorname{Rh}(3)$ & $\mathrm{Cl}(3)$ & $\operatorname{Rh}(4)$ & $-66.8(2)$ \\
\hline $\operatorname{Rh}(4)$ & $\operatorname{Rh}(3)$ & $\mathrm{Cl}(4)$ & $\operatorname{Rh}(4)$ & $0.00(7)$ & $\mathrm{Cl}(3)$ & $\operatorname{Rh}(3)$ & $\mathrm{Cl}(4)$ & $\operatorname{Rh}(4)$ & $-43.4(2)$ \\
\hline C(303) & $\operatorname{Rh}(3)$ & $\mathrm{Cl}(4)$ & $\operatorname{Rh}(4)$ & $146.5(9)$ & $C(306)$ & $\operatorname{Rh}(3)$ & $\mathrm{Cl}(4)$ & $\operatorname{Rh}(4)$ & $57.4(4)$ \\
\hline C(305) & $\operatorname{Rh}(3)$ & $\mathrm{Cl}(4)$ & $\mathrm{Rh}(4)$ & $-154.0(2)$ & $C(302)$ & $\operatorname{Rh}(3)$ & $\mathrm{Cl}(4)$ & $\operatorname{Rh}(4)$ & 107.1(9) \\
\hline $\mathrm{Rh}(4)$ & $\operatorname{Rh}(3)$ & $C(303)$ & $\mathrm{C}(304)$ & $-174.3(5)$ & $\mathrm{Rh}(4)$ & $\operatorname{Rh}(3)$ & $\mathrm{C}(303)$ & $\mathrm{C}(302)$ & $-60.1(1)$ \\
\hline
\end{tabular}




\begin{tabular}{|c|c|c|c|c|c|c|c|c|c|}
\hline $\mathrm{Cl}(3)$ & $\operatorname{Rh}(3)$ & $\mathrm{C}(303)$ & C(304) & $29.8(3)$ & $\mathrm{Cl}(3)$ & $\operatorname{Rh}(3)$ & $C(303)$ & $C(302)$ & $143.9(2)$ \\
\hline $\mathrm{Cl}(4)$ & $\operatorname{Rh}(3)$ & $\mathrm{C}(303)$ & C(304) & $145.2(8)$ & $\mathrm{Cl}(4)$ & $\operatorname{Rh}(3)$ & C(303) & $C(302)$ & $-100.7(8)$ \\
\hline$C(306)$ & $\operatorname{Rh}(3)$ & $\mathrm{C}(303)$ & C(304) & $-49.1(1)$ & $C(306)$ & $\operatorname{Rh}(3)$ & $C(303)$ & $C(302)$ & $65.0(9)$ \\
\hline$C(305)$ & $\operatorname{Rh}(3)$ & $C(303)$ & C(304) & $-10.4(9)$ & $C(305)$ & $\operatorname{Rh}(3)$ & $C(303)$ & $C(302)$ & 103.7(1) \\
\hline$C(302)$ & $\operatorname{Rh}(3)$ & $C(303)$ & C(304) & $-114.1(1)$ & $C(302)$ & $\operatorname{Rh}(3)$ & C(303) & $C(302)$ & $0.0(7)$ \\
\hline $\mathrm{Rh}(4)$ & $\operatorname{Rh}(3)$ & $C(306)$ & $C(305)$ & $-152.0(6)$ & $\mathrm{Rh}(4)$ & $\operatorname{Rh}(3)$ & $C(306)$ & $C(301)$ & $95.8(9)$ \\
\hline $\mathrm{Cl}(3)$ & $\operatorname{Rh}(3)$ & $C(306)$ & $C(305)$ & $-102.3(7)$ & $\mathrm{Cl}(3)$ & $\operatorname{Rh}(3)$ & C(306) & $\mathrm{C}(301)$ & $145.5(8)$ \\
\hline $\mathrm{Cl}(4)$ & $\operatorname{Rh}(3)$ & $C(306)$ & $C(305)$ & $158.5(2)$ & $\mathrm{Cl}(4)$ & $\operatorname{Rh}(3)$ & $C(306)$ & $\mathrm{C}(301)$ & $46.3(3)$ \\
\hline$C(303)$ & $\operatorname{Rh}(3)$ & $C(306)$ & $C(305)$ & $66.8(9)$ & $C(303)$ & $\operatorname{Rh}(3)$ & $C(306)$ & $C(301)$ & $-45.3(1)$ \\
\hline$C(305)$ & $\operatorname{Rh}(3)$ & $C(306)$ & $C(305)$ & $0.0(6)$ & $C(305)$ & $\mathrm{Rh}(3)$ & $C(306)$ & $C(301)$ & $-112.1(1)$ \\
\hline$C(302)$ & $\operatorname{Rh}(3)$ & C(306) & C(305) & 104.9(1) & $C(302)$ & $\operatorname{Rh}(3)$ & C(306) & $C(301)$ & $-7.2(9)$ \\
\hline $\operatorname{Rh}(4)$ & $\operatorname{Rh}(3)$ & $C(305)$ & $C(306)$ & $56.0(1)$ & $\mathrm{Rh}(4)$ & $\operatorname{Rh}(3)$ & $C(305)$ & $C(304)$ & $168.1(6)$ \\
\hline $\operatorname{Rh}(4)$ & $\operatorname{Rh}(3)$ & $C(305)$ & $C(315)$ & $-62.7(2)$ & $\mathrm{Cl}(3)$ & $\operatorname{Rh}(3)$ & $C(305)$ & $C(306)$ & $85.3(8)$ \\
\hline $\mathrm{Cl}(3)$ & $\operatorname{Rh}(3)$ & $C(305)$ & C(304) & $-162.6(8)$ & $\mathrm{Cl}(3)$ & $\operatorname{Rh}(3)$ & $C(305)$ & $C(315)$ & $-33.4(1)$ \\
\hline $\mathrm{Cl}(4)$ & $\operatorname{Rh}(3)$ & $C(305)$ & C(306) & $-168.4(8)$ & $\mathrm{Cl}(4)$ & $\operatorname{Rh}(3)$ & $C(305)$ & $C(304)$ & $-56.3(2)$ \\
\hline $\mathrm{Cl}(4)$ & $\operatorname{Rh}(3)$ & $C(305)$ & $C(315)$ & $72.9(2)$ & $C(303)$ & $\operatorname{Rh}(3)$ & $C(305)$ & $C(306)$ & $-101.9(1)$ \\
\hline C(303) & $\operatorname{Rh}(3)$ & $C(305)$ & C(304) & $10.2(9)$ & $C(303)$ & $\operatorname{Rh}(3)$ & $C(305)$ & $C(315)$ & 139.4(1) \\
\hline$C(306)$ & $\operatorname{Rh}(3)$ & $C(305)$ & C(306) & $0.0(6)$ & $C(306)$ & $\operatorname{Rh}(3)$ & $C(305)$ & $C(304)$ & 112.1(1) \\
\hline$C(306)$ & $\operatorname{Rh}(3)$ & $C(305)$ & $C(315)$ & $-118.7(2)$ & $C(302)$ & $\operatorname{Rh}(3)$ & $C(305)$ & $C(306)$ & $-63.9(9)$ \\
\hline$C(302)$ & $\operatorname{Rh}(3)$ & $C(305)$ & C(304) & 48.2(1) & $C(302)$ & $\operatorname{Rh}(3)$ & $C(305)$ & $C(315)$ & 177.3(1) \\
\hline $\operatorname{Rh}(4)$ & $\operatorname{Rh}(3)$ & $C(302)$ & $C(303)$ & $141.7(7)$ & $\mathrm{Rh}(4)$ & $\operatorname{Rh}(3)$ & $C(302)$ & $\mathrm{C}(309)$ & $22.2(1)$ \\
\hline $\operatorname{Rh}(4)$ & $\operatorname{Rh}(3)$ & $C(302)$ & C(301) & $-107.7(8)$ & $\mathrm{Cl}(3)$ & $\operatorname{Rh}(3)$ & $C(302)$ & $C(303)$ & $-167.2(7)$ \\
\hline $\mathrm{Cl}(3)$ & $\operatorname{Rh}(3)$ & $\mathrm{C}(302)$ & C(309) & $73.3(2)$ & $\mathrm{Cl}(3)$ & $\operatorname{Rh}(3)$ & $C(302)$ & $C(301)$ & $-56.6(2)$ \\
\hline $\mathrm{Cl}(4)$ & $\operatorname{Rh}(3)$ & $\mathrm{C}(302)$ & $C(303)$ & $88.2(8)$ & $\mathrm{Cl}(4)$ & $\operatorname{Rh}(3)$ & $C(302)$ & $\mathrm{C}(309)$ & $-31.4(1)$ \\
\hline $\mathrm{Cl}(4)$ & $\operatorname{Rh}(3)$ & $C(302)$ & C(301) & $-161.2(8)$ & $C(303)$ & $\operatorname{Rh}(3)$ & $C(302)$ & $C(303)$ & $0.0(7)$ \\
\hline$C(303)$ & $\operatorname{Rh}(3)$ & $\mathrm{C}(302)$ & C(309) & $-119.5(2)$ & $C(303)$ & $\operatorname{Rh}(3)$ & $C(302)$ & $C(301)$ & $110.6(1)$ \\
\hline$C(306)$ & $\operatorname{Rh}(3)$ & $C(302)$ & C(303) & $-103.5(1)$ & $C(306)$ & $\operatorname{Rh}(3)$ & $C(302)$ & $\mathrm{C}(309)$ & $137.0(1)$ \\
\hline$C(306)$ & $\operatorname{Rh}(3)$ & $C(302)$ & C(301) & $7.2(9)$ & $C(305)$ & $\operatorname{Rh}(3)$ & $C(302)$ & $C(303)$ & $-65.5(9)$ \\
\hline$C(305)$ & $\operatorname{Rh}(3)$ & $\mathrm{C}(302)$ & C(309) & $175.0(1)$ & $C(305)$ & $\operatorname{Rh}(3)$ & $C(302)$ & $C(301)$ & 45.1(1) \\
\hline $\operatorname{Rh}(3)$ & $\operatorname{Rh}(4)$ & $\mathrm{Cl}(3)$ & $\operatorname{Rh}(3)$ & $0.00(7)$ & $\mathrm{Cl}(4)$ & $\operatorname{Rh}(4)$ & $\mathrm{Cl}(3)$ & $\operatorname{Rh}(3)$ & $-43.8(2)$ \\
\hline$C(403)$ & $\operatorname{Rh}(4)$ & $\mathrm{Cl}(3)$ & $\operatorname{Rh}(3)$ & $152.6(8)$ & $C(405)$ & $\operatorname{Rh}(4)$ & $\mathrm{Cl}(3)$ & $\operatorname{Rh}(3)$ & $-151.9(3)$ \\
\hline C(406) & $\operatorname{Rh}(4)$ & $\mathrm{Cl}(3)$ & $\operatorname{Rh}(3)$ & $61.4(3)$ & $C(402)$ & $\operatorname{Rh}(4)$ & $\mathrm{Cl}(3)$ & $\operatorname{Rh}(3)$ & $112.7(8)$ \\
\hline $\operatorname{Rh}(3)$ & $\operatorname{Rh}(4)$ & $\mathrm{Cl}(4)$ & $\operatorname{Rh}(3)$ & $0.00(7)$ & $\mathrm{Cl}(3)$ & $\operatorname{Rh}(4)$ & $\mathrm{Cl}(4)$ & $\operatorname{Rh}(3)$ & $43.4(2)$ \\
\hline$C(403)$ & $\operatorname{Rh}(4)$ & $\mathrm{Cl}(4)$ & $\operatorname{Rh}(3)$ & $157.3(3)$ & $C(405)$ & $\operatorname{Rh}(4)$ & $\mathrm{Cl}(4)$ & $\operatorname{Rh}(3)$ & $-156.0(8)$ \\
\hline$C(406)$ & $\operatorname{Rh}(4)$ & $\mathrm{Cl}(4)$ & $\operatorname{Rh}(3)$ & $-116.5(8)$ & $C(402)$ & $\operatorname{Rh}(4)$ & $\mathrm{Cl}(4)$ & $\operatorname{Rh}(3)$ & $-64.0(2)$ \\
\hline $\operatorname{Rh}(3)$ & $\operatorname{Rh}(4)$ & C(403) & C(402) & $-56.3(1)$ & $\operatorname{Rh}(3)$ & $\operatorname{Rh}(4)$ & C(403) & $C(404)$ & $-169.6(5)$ \\
\hline $\mathrm{Cl}(3)$ & $\operatorname{Rh}(4)$ & C(403) & C(402) & $-93.7(7)$ & $\mathrm{Cl}(3)$ & $\operatorname{Rh}(4)$ & $C(403)$ & $C(404)$ & $153.0(7)$ \\
\hline $\mathrm{Cl}(4)$ & $\operatorname{Rh}(4)$ & C(403) & C(402) & 154.7(1) & $\mathrm{Cl}(4)$ & $\operatorname{Rh}(4)$ & C(403) & $C(404)$ & $41.3(2)$ \\
\hline$C(405)$ & $\operatorname{Rh}(4)$ & C(403) & C(402) & 104.1(9) & $C(405)$ & $\operatorname{Rh}(4)$ & C(403) & $C(404)$ & $-9.2(8)$ \\
\hline C(406) & $\operatorname{Rh}(4)$ & C(403) & C(402) & $65.3(9)$ & $C(406)$ & $\operatorname{Rh}(4)$ & $C(403)$ & $C(404)$ & $-48.0(9)$ \\
\hline$C(402)$ & $\operatorname{Rh}(4)$ & C(403) & C(402) & $0.0(6)$ & $C(402)$ & $\operatorname{Rh}(4)$ & C(403) & $C(404)$ & $-113.3(1)$ \\
\hline $\operatorname{Rh}(3)$ & $\operatorname{Rh}(4)$ & $C(405)$ & C(406) & $58.2(1)$ & $\operatorname{Rh}(3)$ & $\operatorname{Rh}(4)$ & $C(405)$ & $C(415)$ & $-61.7(1)$ \\
\hline $\operatorname{Rh}(3)$ & $\operatorname{Rh}(4)$ & $C(405)$ & C(404) & $168.8(5)$ & $\mathrm{Cl}(3)$ & $\operatorname{Rh}(4)$ & $C(405)$ & $C(406)$ & $-162.1(1)$ \\
\hline $\mathrm{Cl}(3)$ & $\operatorname{Rh}(4)$ & C(405) & C(415) & $78.0(2)$ & $\mathrm{Cl}(3)$ & $\operatorname{Rh}(4)$ & C(405) & $C(404)$ & $-51.5(2)$ \\
\hline $\mathrm{Cl}(4)$ & $\operatorname{Rh}(4)$ & $C(405)$ & C(406) & $92.4(7)$ & $\mathrm{Cl}(4)$ & $\operatorname{Rh}(4)$ & $C(405)$ & $C(415)$ & $-27.5(1)$ \\
\hline $\mathrm{Cl}(4)$ & $\operatorname{Rh}(4)$ & $C(405)$ & C(404) & $-157.0(7)$ & $C(403)$ & $\mathrm{Rh}(4)$ & $C(405)$ & $C(406)$ & $-101.3(9)$ \\
\hline C(403) & $\operatorname{Rh}(4)$ & C(405) & C(415) & 138.7(1) & $C(403)$ & $\operatorname{Rh}(4)$ & C(405) & $C(404)$ & $9.2(8)$ \\
\hline C(406) & $\operatorname{Rh}(4)$ & C(405) & C(406) & $0.0(6)$ & $C(406)$ & $\operatorname{Rh}(4)$ & $C(405)$ & $C(415)$ & $-120.0(1)$ \\
\hline C(406) & $\operatorname{Rh}(4)$ & $C(405)$ & C(404) & $110.5(1)$ & $C(402)$ & $\operatorname{Rh}(4)$ & $C(405)$ & $C(406)$ & $-63.2(8)$ \\
\hline$C(402)$ & $\operatorname{Rh}(4)$ & C(405) & C(415) & $176.9(1)$ & $C(402)$ & $\operatorname{Rh}(4)$ & C(405) & $C(404)$ & 47.4(9) \\
\hline $\operatorname{Rh}(3)$ & $\operatorname{Rh}(4)$ & C(406) & $C(401)$ & $98.9(8)$ & $\operatorname{Rh}(3)$ & $\operatorname{Rh}(4)$ & $C(406)$ & $C(405)$ & $-147.1(6)$ \\
\hline $\mathrm{Cl}(3)$ & $\operatorname{Rh}(4)$ & C(406) & C(401) & $48.5(2)$ & $\mathrm{Cl}(3)$ & $\operatorname{Rh}(4)$ & C(406) & $C(405)$ & $162.5(1)$ \\
\hline $\mathrm{Cl}(4)$ & $\operatorname{Rh}(4)$ & $C(406)$ & $C(401)$ & $151.3(8)$ & $\mathrm{Cl}(4)$ & $\operatorname{Rh}(4)$ & C(406) & $C(405)$ & $-94.7(7)$ \\
\hline
\end{tabular}




\begin{tabular}{|c|c|c|c|c|c|c|c|c|c|}
\hline$C(403)$ & $\operatorname{Rh}(4)$ & $C(406)$ & $\mathrm{C}(401)$ & $-46.6(1)$ & C(403) & $\operatorname{Rh}(4)$ & $C(406)$ & $C(405)$ & $67.3(9)$ \\
\hline C(405) & $\operatorname{Rh}(4)$ & $C(406)$ & $\mathrm{C}(401)$ & $-114.0(1)$ & $\mathrm{C}(405)$ & $\operatorname{Rh}(4)$ & $C(406)$ & $C(405)$ & $0.0(6)$ \\
\hline C(402) & $\operatorname{Rh}(4)$ & $C(406)$ & $\mathrm{C}(401)$ & $-8.0(8)$ & $C(402)$ & $\operatorname{Rh}(4)$ & C(406) & $\mathrm{C}(405)$ & $106.0(9)$ \\
\hline $\operatorname{Rh}(3)$ & $\operatorname{Rh}(4)$ & $C(402)$ & $\mathrm{C}(403)$ & $147.1(6)$ & $\operatorname{Rh}(3)$ & $\operatorname{Rh}(4)$ & $C(402)$ & $\mathrm{C}(401)$ & $-102.0(8)$ \\
\hline $\operatorname{Rh}(3)$ & $\operatorname{Rh}(4)$ & $C(402)$ & $\mathrm{C}(409)$ & $28.2(1)$ & $\mathrm{Cl}(3)$ & $\operatorname{Rh}(4)$ & $\mathrm{C}(402)$ & $\mathrm{C}(403)$ & $94.5(7)$ \\
\hline $\mathrm{Cl}(3)$ & $\operatorname{Rh}(4)$ & $C(402)$ & $\mathrm{C}(401)$ & $-154.7(7)$ & $\mathrm{Cl}(3)$ & $\operatorname{Rh}(4)$ & C(402) & $\mathrm{C}(409)$ & $-24.4(1)$ \\
\hline $\mathrm{Cl}(4)$ & $\operatorname{Rh}(4)$ & $C(402)$ & $\mathrm{C}(403)$ & $-161.5(9)$ & $\mathrm{Cl}(4)$ & $\operatorname{Rh}(4)$ & $\mathrm{C}(402)$ & $\mathrm{C}(401)$ & $-50.7(2)$ \\
\hline $\mathrm{Cl}(4)$ & $\operatorname{Rh}(4)$ & $\mathrm{C}(402)$ & $\mathrm{C}(409)$ & $79.6(2)$ & $C(403)$ & $\operatorname{Rh}(4)$ & C(402) & $\mathrm{C}(403)$ & $0.0(6)$ \\
\hline$C(403)$ & $\mathrm{Rh}(4)$ & $\mathrm{C}(402)$ & $\mathrm{C}(401)$ & $110.8(1)$ & C(403) & $\operatorname{Rh}(4)$ & $\mathrm{C}(402)$ & C(409) & $-118.9(1)$ \\
\hline$C(405)$ & $\operatorname{Rh}(4)$ & $\mathrm{C}(402)$ & $\mathrm{C}(403)$ & $-65.3(9)$ & $C(405)$ & $\operatorname{Rh}(4)$ & $\mathrm{C}(402)$ & $\mathrm{C}(401)$ & $45.5(9)$ \\
\hline $\mathrm{C}(405)$ & $\operatorname{Rh}(4)$ & $\mathrm{C}(402)$ & $\mathrm{C}(409)$ & $175.8(1)$ & C(406) & $\operatorname{Rh}(4)$ & C(402) & $\mathrm{C}(403)$ & $-103.0(9)$ \\
\hline C(406) & $\mathrm{Rh}(4)$ & $\mathrm{C}(402)$ & $\mathrm{C}(401)$ & $7.9(8)$ & C(406) & $\operatorname{Rh}(4)$ & $C(402)$ & $\mathrm{C}(409)$ & 138.1(1) \\
\hline $\operatorname{Rh}(5)$ & $\operatorname{Rh}(6)$ & $\mathrm{Cl}(6)$ & $\operatorname{Rh}(5)$ & $0.00(7)$ & $\mathrm{Cl}(5)$ & $\operatorname{Rh}(6)$ & $\mathrm{Cl}(6)$ & $\operatorname{Rh}(5)$ & $-46.2(2)$ \\
\hline$C(605)$ & $\operatorname{Rh}(6)$ & $\mathrm{Cl}(6)$ & $\operatorname{Rh}(5)$ & $108.0(8)$ & $C(602)$ & $\operatorname{Rh}(6)$ & $\mathrm{Cl}(6)$ & $\operatorname{Rh}(5)$ & $-156.6(3)$ \\
\hline $\mathrm{C}(603)$ & $\operatorname{Rh}(6)$ & $\mathrm{Cl}(6)$ & $\operatorname{Rh}(5)$ & $57.5(3)$ & C(606) & $\operatorname{Rh}(6)$ & $\mathrm{Cl}(6)$ & $\operatorname{Rh}(5)$ & $147.7(9)$ \\
\hline $\operatorname{Rh}(5)$ & $\operatorname{Rh}(6)$ & $\mathrm{Cl}(5)$ & $\operatorname{Rh}(5)$ & $0.00(7)$ & $\mathrm{Cl}(6)$ & $\operatorname{Rh}(6)$ & $\mathrm{Cl}(5)$ & $\operatorname{Rh}(5)$ & $46.2(2)$ \\
\hline$C(605)$ & $\operatorname{Rh}(6)$ & $\mathrm{Cl}(5)$ & $\operatorname{Rh}(5)$ & $-61.1(2)$ & $C(602)$ & $\operatorname{Rh}(6)$ & $\mathrm{Cl}(5)$ & $\operatorname{Rh}(5)$ & $-155.1(9)$ \\
\hline $\mathrm{C}(603)$ & $\operatorname{Rh}(6)$ & $\mathrm{Cl}(5)$ & $\operatorname{Rh}(5)$ & $-115.7(8)$ & C(606) & $\operatorname{Rh}(6)$ & $\mathrm{Cl}(5)$ & $\operatorname{Rh}(5)$ & $159.6(4)$ \\
\hline $\operatorname{Rh}(5)$ & $\operatorname{Rh}(6)$ & $\mathrm{C}(605)$ & $C(604)$ & $-102.2(8)$ & $\operatorname{Rh}(5)$ & $\operatorname{Rh}(6)$ & $C(605)$ & $\mathrm{C}(606)$ & $146.8(6)$ \\
\hline $\operatorname{Rh}(5)$ & $\operatorname{Rh}(6)$ & $\mathrm{C}(605)$ & $\mathrm{C}(615)$ & $26.8(1)$ & $\mathrm{Cl}(6)$ & $\operatorname{Rh}(6)$ & $C(605)$ & $\mathrm{C}(604)$ & $-156.8(7)$ \\
\hline $\mathrm{Cl}(6)$ & $\operatorname{Rh}(6)$ & $\mathrm{C}(605)$ & $\mathrm{C}(606)$ & $92.2(7)$ & $\mathrm{Cl}(6)$ & $\operatorname{Rh}(6)$ & $C(605)$ & $\mathrm{C}(615)$ & $-27.8(1)$ \\
\hline $\mathrm{Cl}(5)$ & $\operatorname{Rh}(6)$ & $\mathrm{C}(605)$ & $\mathrm{C}(604)$ & $-53.4(2)$ & $\mathrm{Cl}(5)$ & $\operatorname{Rh}(6)$ & $C(605)$ & $\mathrm{C}(606)$ & $-164.4(8)$ \\
\hline $\mathrm{Cl}(5)$ & $\operatorname{Rh}(6)$ & $\mathrm{C}(605)$ & $\mathrm{C}(615)$ & $75.6(2)$ & $C(602)$ & $\operatorname{Rh}(6)$ & $\mathrm{C}(605)$ & $\mathrm{C}(604)$ & $45.6(1)$ \\
\hline$C(602)$ & $\operatorname{Rh}(6)$ & $\mathrm{C}(605)$ & $C(606)$ & $-65.4(9)$ & $C(602)$ & $\operatorname{Rh}(6)$ & $C(605)$ & $\mathrm{C}(615)$ & $174.6(1)$ \\
\hline$C(603)$ & $\operatorname{Rh}(6)$ & $\mathrm{C}(605)$ & $\mathrm{C}(604)$ & $7.8(9)$ & $C(603)$ & $\operatorname{Rh}(6)$ & $C(605)$ & $\mathrm{C}(606)$ & $-103.2(1)$ \\
\hline$C(603)$ & $\operatorname{Rh}(6)$ & $\mathrm{C}(605)$ & $\mathrm{C}(615)$ & $136.8(1)$ & $C(606)$ & $\operatorname{Rh}(6)$ & $C(605)$ & $\mathrm{C}(604)$ & $111.0(1)$ \\
\hline C(606) & $\operatorname{Rh}(6)$ & $\mathrm{C}(605)$ & $\mathrm{C}(606)$ & $0.0(6)$ & $C(606)$ & $\operatorname{Rh}(6)$ & $\mathrm{C}(605)$ & $\mathrm{C}(615)$ & $-120.0(1)$ \\
\hline $\operatorname{Rh}(5)$ & $\operatorname{Rh}(6)$ & $\mathrm{C}(602)$ & $\mathrm{C}(601)$ & $163.5(5)$ & $\operatorname{Rh}(5)$ & $\operatorname{Rh}(6)$ & $\mathrm{C}(602)$ & $\mathrm{C}(603)$ & $51.6(1)$ \\
\hline $\mathrm{Rh}(5)$ & $\operatorname{Rh}(6)$ & $\mathrm{C}(602)$ & $\mathrm{C}(609)$ & $-67.0(1)$ & $\mathrm{Cl}(6)$ & $\operatorname{Rh}(6)$ & $C(602)$ & $\mathrm{C}(601)$ & $-51.6(2)$ \\
\hline $\mathrm{Cl}(6)$ & $\operatorname{Rh}(6)$ & $\mathrm{C}(602)$ & $\mathrm{C}(603)$ & $-163.5(9)$ & $\mathrm{Cl}(6)$ & $\operatorname{Rh}(6)$ & $C(602)$ & $\mathrm{C}(609)$ & $77.9(2)$ \\
\hline $\mathrm{Cl}(5)$ & $\operatorname{Rh}(6)$ & $\mathrm{C}(602)$ & $\mathrm{C}(601)$ & $-158.8(8)$ & $\mathrm{Cl}(5)$ & $\operatorname{Rh}(6)$ & $C(602)$ & $\mathrm{C}(603)$ & $89.3(7)$ \\
\hline $\mathrm{Cl}(5)$ & $\operatorname{Rh}(6)$ & $\mathrm{C}(602)$ & $\mathrm{C}(609)$ & $-29.2(1)$ & $C(605)$ & $\operatorname{Rh}(6)$ & $\mathrm{C}(602)$ & $\mathrm{C}(601)$ & 47.9(1) \\
\hline$C(605)$ & $\operatorname{Rh}(6)$ & $\mathrm{C}(602)$ & $C(603)$ & $-64.0(9)$ & $C(605)$ & $\operatorname{Rh}(6)$ & $C(602)$ & $\mathrm{C}(609)$ & $177.5(1)$ \\
\hline$C(603)$ & $\operatorname{Rh}(6)$ & $C(602)$ & $\mathrm{C}(601)$ & 111.9(1) & $C(603)$ & $\operatorname{Rh}(6)$ & $C(602)$ & $C(603)$ & $0.0(6)$ \\
\hline$C(603)$ & $\operatorname{Rh}(6)$ & $C(602)$ & $\mathrm{C}(609)$ & $-118.6(1)$ & $C(606)$ & $\operatorname{Rh}(6)$ & $C(602)$ & $\mathrm{C}(601)$ & 9.7(9) \\
\hline$C(606)$ & $\operatorname{Rh}(6)$ & $C(602)$ & $C(603)$ & $-102.2(1)$ & $C(606)$ & $\operatorname{Rh}(6)$ & $C(602)$ & $\mathrm{C}(609)$ & 139.3(1) \\
\hline $\operatorname{Rh}(5)$ & $\operatorname{Rh}(6)$ & $C(603)$ & $\mathrm{C}(604)$ & $95.8(8)$ & $\operatorname{Rh}(5)$ & $\operatorname{Rh}(6)$ & $C(603)$ & $\mathrm{C}(602)$ & $-151.3(6)$ \\
\hline $\mathrm{Cl}(6)$ & $\operatorname{Rh}(6)$ & $C(603)$ & $C(604)$ & $47.5(2)$ & $\mathrm{Cl}(6)$ & $\operatorname{Rh}(6)$ & $C(603)$ & $C(602)$ & 160.3(1) \\
\hline $\mathrm{Cl}(5)$ & $\operatorname{Rh}(6)$ & $C(603)$ & $C(604)$ & $149.0(8)$ & $\mathrm{Cl}(5)$ & $\operatorname{Rh}(6)$ & $C(603)$ & $C(602)$ & $-98.2(7)$ \\
\hline$C(605)$ & $\operatorname{Rh}(6)$ & $C(603)$ & $\mathrm{C}(604)$ & $-7.8(9)$ & $C(605)$ & $\operatorname{Rh}(6)$ & $C(603)$ & $C(602)$ & $105.0(1)$ \\
\hline$C(602)$ & $\operatorname{Rh}(6)$ & $C(603)$ & $\mathrm{C}(604)$ & $-112.8(1)$ & $C(602)$ & $\operatorname{Rh}(6)$ & $C(603)$ & $\mathrm{C}(602)$ & $0.0(6)$ \\
\hline$C(606)$ & $\operatorname{Rh}(6)$ & $C(603)$ & $C(604)$ & $-46.2(1)$ & $C(606)$ & $\operatorname{Rh}(6)$ & $C(603)$ & $C(602)$ & 66.6(9) \\
\hline $\operatorname{Rh}(5)$ & $\operatorname{Rh}(6)$ & $\mathrm{C}(606)$ & $C(605)$ & $-53.6(1)$ & $\operatorname{Rh}(5)$ & $\operatorname{Rh}(6)$ & $C(606)$ & $\mathrm{C}(601)$ & $-167.1(5)$ \\
\hline $\mathrm{Cl}(6)$ & $\operatorname{Rh}(6)$ & $\mathrm{C}(606)$ & $\mathrm{C}(605)$ & $-96.0(7)$ & $\mathrm{Cl}(6)$ & $\operatorname{Rh}(6)$ & C(606) & $\mathrm{C}(601)$ & $150.6(8)$ \\
\hline $\mathrm{Cl}(5)$ & $\operatorname{Rh}(6)$ & $\mathrm{C}(606)$ & $\mathrm{C}(605)$ & $152.5(2)$ & $\mathrm{Cl}(5)$ & $\operatorname{Rh}(6)$ & C(606) & $\mathrm{C}(601)$ & $39.1(3)$ \\
\hline$C(605)$ & $\operatorname{Rh}(6)$ & $\mathrm{C}(606)$ & $\mathrm{C}(605)$ & $0.0(6)$ & $C(605)$ & $\operatorname{Rh}(6)$ & $\mathrm{C}(606)$ & $\mathrm{C}(601)$ & $-113.4(1)$ \\
\hline $\mathrm{C}(602)$ & $\operatorname{Rh}(6)$ & $\mathrm{C}(606)$ & $\mathrm{C}(605)$ & $103.7(1)$ & $C(602)$ & $\operatorname{Rh}(6)$ & C(606) & $\mathrm{C}(601)$ & $-9.7(9)$ \\
\hline $\mathrm{C}(603)$ & $\operatorname{Rh}(6)$ & $\mathrm{C}(606)$ & $\mathrm{C}(605)$ & $65.2(9)$ & $\mathrm{C}(603)$ & $\operatorname{Rh}(6)$ & C(606) & $\mathrm{C}(601)$ & $-48.2(1)$ \\
\hline $\operatorname{Rh}(2)$ & $\operatorname{Rh}(1)$ & $\mathrm{Cl}(2)$ & $\operatorname{Rh}(2)$ & $0.00(8)$ & $\mathrm{Cl}(1)$ & $\operatorname{Rh}(1)$ & $\mathrm{Cl}(2)$ & $\mathrm{Rh}(2)$ & $48.5(2)$ \\
\hline$C(102)$ & $\operatorname{Rh}(1)$ & $\mathrm{Cl}(2)$ & $\operatorname{Rh}(2)$ & $-149.8(1)$ & $C(103)$ & $\operatorname{Rh}(1)$ & $\mathrm{Cl}(2)$ & $\operatorname{Rh}(2)$ & $-111.0(8)$ \\
\hline C(106) & $\operatorname{Rh}(1)$ & $\mathrm{Cl}(2)$ & $\operatorname{Rh}(2)$ & $171.2(7)$ & $\mathrm{C}(105)$ & $\operatorname{Rh}(1)$ & $\mathrm{Cl}(2)$ & $\operatorname{Rh}(2)$ & $-51.5(2)$ \\
\hline $\operatorname{Rh}(2)$ & $\operatorname{Rh}(1)$ & $\mathrm{Cl}(1)$ & $\operatorname{Rh}(2)$ & $0.00(8)$ & $\mathrm{Cl}(2)$ & $\operatorname{Rh}(1)$ & $\mathrm{Cl}(1)$ & $\operatorname{Rh}(2)$ & $-48.4(2)$ \\
\hline$C(102)$ & $\operatorname{Rh}(1)$ & $\mathrm{Cl}(1)$ & $\operatorname{Rh}(2)$ & $-167.7(3)$ & $C(103)$ & $\operatorname{Rh}(1)$ & $\mathrm{Cl}(1)$ & $\operatorname{Rh}(2)$ & 49.1(3) \\
\hline
\end{tabular}




\begin{tabular}{|c|c|c|c|c|c|c|c|c|c|}
\hline$C(106)$ & $\mathrm{Rh}(1)$ & $\mathrm{Cl}(1)$ & $\operatorname{Rh}(2)$ & $140.3(1)$ & $C(105)$ & $\operatorname{Rh}(1)$ & $\mathrm{Cl}(1)$ & $\operatorname{Rh}(2)$ & $101.2(8)$ \\
\hline $\operatorname{Rh}(2)$ & $\operatorname{Rh}(1)$ & $C(102)$ & $\mathrm{C}(109)$ & $-80.3(2)$ & $\operatorname{Rh}(2)$ & $\operatorname{Rh}(1)$ & $C(102)$ & $\mathrm{C}(103)$ & $37.5(1)$ \\
\hline $\operatorname{Rh}(2)$ & $\operatorname{Rh}(1)$ & $C(102)$ & $\mathrm{C}(101)$ & $148.1(7)$ & $\mathrm{Cl}(2)$ & $\operatorname{Rh}(1)$ & $C(102)$ & $\mathrm{C}(109)$ & $-33.8(1)$ \\
\hline $\mathrm{Cl}(2)$ & $\operatorname{Rh}(1)$ & $C(102)$ & $C(103)$ & $84.1(8)$ & $\mathrm{Cl}(2)$ & $\operatorname{Rh}(1)$ & $C(102)$ & $C(101)$ & $-165.3(8)$ \\
\hline $\mathrm{Cl}(1)$ & $\operatorname{Rh}(1)$ & $C(102)$ & C(109) & $81.8(2)$ & $\mathrm{Cl}(1)$ & $\operatorname{Rh}(1)$ & $C(102)$ & $\mathrm{C}(103)$ & $-160.3(1)$ \\
\hline $\mathrm{Cl}(1)$ & $\operatorname{Rh}(1)$ & $C(102)$ & $\mathrm{C}(101)$ & $-49.8(2)$ & $C(103)$ & $\mathrm{Rh}(1)$ & $C(102)$ & C(109) & $-117.9(2)$ \\
\hline$C(103)$ & $\operatorname{Rh}(1)$ & $C(102)$ & C(103) & $0.0(6)$ & $C(103)$ & $\operatorname{Rh}(1)$ & $C(102)$ & $\mathrm{C}(101)$ & $110.6(1)$ \\
\hline C(106) & $\operatorname{Rh}(1)$ & $C(102)$ & C(109) & $139.3(2)$ & $C(106)$ & $\operatorname{Rh}(1)$ & $C(102)$ & $C(103)$ & $-102.8(1)$ \\
\hline C(106) & $\operatorname{Rh}(1)$ & $C(102)$ & $C(101)$ & $7.7(1)$ & $C(105)$ & $\operatorname{Rh}(1)$ & $C(102)$ & $\mathrm{C}(109)$ & 176.7(1) \\
\hline$C(105)$ & $\operatorname{Rh}(1)$ & $C(102)$ & $C(103)$ & $-65.5(9)$ & $C(105)$ & $\operatorname{Rh}(1)$ & $C(102)$ & $\mathrm{C}(101)$ & 45.1(1) \\
\hline $\operatorname{Rh}(2)$ & $\operatorname{Rh}(1)$ & $C(103)$ & $C(102)$ & $-158.6(6)$ & $\operatorname{Rh}(2)$ & $\operatorname{Rh}(1)$ & $C(103)$ & $C(104)$ & $88.1(8)$ \\
\hline $\mathrm{Cl}(2)$ & $\operatorname{Rh}(1)$ & $C(103)$ & $C(102)$ & $-104.8(8)$ & $\mathrm{Cl}(2)$ & $\operatorname{Rh}(1)$ & $C(103)$ & $C(104)$ & $141.9(8)$ \\
\hline $\mathrm{Cl}(1)$ & $\operatorname{Rh}(1)$ & $C(103)$ & $C(102)$ & $160.6(1)$ & $\mathrm{Cl}(1)$ & $\operatorname{Rh}(1)$ & $C(103)$ & $C(104)$ & $47.3(2)$ \\
\hline$C(102)$ & $\operatorname{Rh}(1)$ & $C(103)$ & $C(102)$ & $0.0(7)$ & $C(102)$ & $\operatorname{Rh}(1)$ & $C(103)$ & $\mathrm{C}(104)$ & $-113.3(1)$ \\
\hline$C(106)$ & $\operatorname{Rh}(1)$ & $C(103)$ & $C(102)$ & $65.1(1)$ & $C(106)$ & $\operatorname{Rh}(1)$ & $C(103)$ & $C(104)$ & $-48.1(1)$ \\
\hline$C(105)$ & $\operatorname{Rh}(1)$ & $C(103)$ & $C(102)$ & $103.4(1)$ & $C(105)$ & $\operatorname{Rh}(1)$ & $C(103)$ & $\mathrm{C}(104)$ & $-9.9(9)$ \\
\hline $\operatorname{Rh}(2)$ & $\operatorname{Rh}(1)$ & $C(106)$ & $\mathrm{C}(101)$ & $-156.7(6)$ & $\operatorname{Rh}(2)$ & $\operatorname{Rh}(1)$ & $C(106)$ & $C(105)$ & $-43.8(1)$ \\
\hline $\mathrm{Cl}(2)$ & $\operatorname{Rh}(1)$ & $C(106)$ & $C(101)$ & $33.6(4)$ & $\mathrm{Cl}(2)$ & $\operatorname{Rh}(1)$ & $C(106)$ & $C(105)$ & $146.5(3)$ \\
\hline $\mathrm{Cl}(1)$ & $\operatorname{Rh}(1)$ & $C(106)$ & $C(101)$ & $154.9(9)$ & $\mathrm{Cl}(1)$ & $\operatorname{Rh}(1)$ & $C(106)$ & $C(105)$ & $-92.3(8)$ \\
\hline$C(102)$ & $\operatorname{Rh}(1)$ & $C(106)$ & $C(101)$ & $-7.8(1)$ & $C(102)$ & $\operatorname{Rh}(1)$ & $C(106)$ & C(105) & $105.0(1)$ \\
\hline$C(103)$ & $\operatorname{Rh}(1)$ & $C(106)$ & $C(101)$ & $-45.9(1)$ & $C(103)$ & $\operatorname{Rh}(1)$ & $C(106)$ & $C(105)$ & $66.9(1)$ \\
\hline$C(105)$ & $\operatorname{Rh}(1)$ & $C(106)$ & $C(101)$ & $-112.8(1)$ & $C(105)$ & $\operatorname{Rh}(1)$ & $C(106)$ & $C(105)$ & $0.0(7)$ \\
\hline $\operatorname{Rh}(2)$ & $\operatorname{Rh}(1)$ & $C(105)$ & $C(104)$ & $-98.0(8)$ & $\operatorname{Rh}(2)$ & $\operatorname{Rh}(1)$ & $C(105)$ & C(106) & $150.5(7)$ \\
\hline $\operatorname{Rh}(2)$ & $\operatorname{Rh}(1)$ & $C(105)$ & $C(115)$ & $32.4(1)$ & $\mathrm{Cl}(2)$ & $\operatorname{Rh}(1)$ & $C(105)$ & $C(104)$ & $-57.5(2)$ \\
\hline $\mathrm{Cl}(2)$ & $\operatorname{Rh}(1)$ & $C(105)$ & $C(106)$ & $-168.9(8)$ & $\mathrm{Cl}(2)$ & $\operatorname{Rh}(1)$ & $C(105)$ & $C(115)$ & $72.9(2)$ \\
\hline $\mathrm{Cl}(1)$ & $\operatorname{Rh}(1)$ & $C(105)$ & C(104) & $-152.6(8)$ & $\mathrm{Cl}(1)$ & $\operatorname{Rh}(1)$ & $C(105)$ & $C(106)$ & $95.9(8)$ \\
\hline $\mathrm{Cl}(1)$ & $\operatorname{Rh}(1)$ & $C(105)$ & $C(115)$ & $-22.2(1)$ & $C(102)$ & $\operatorname{Rh}(1)$ & $C(105)$ & $C(104)$ & 47.9(1) \\
\hline$C(102)$ & $\operatorname{Rh}(1)$ & $C(105)$ & $C(106)$ & $-63.6(1)$ & $C(102)$ & $\operatorname{Rh}(1)$ & $C(105)$ & $C(115)$ & 178.3(1) \\
\hline$C(103)$ & $\operatorname{Rh}(1)$ & $C(105)$ & $C(104)$ & 10.0(9) & $C(103)$ & $\operatorname{Rh}(1)$ & $C(105)$ & $C(106)$ & $-101.4(1)$ \\
\hline$C(103)$ & $\operatorname{Rh}(1)$ & $C(105)$ & $C(115)$ & $140.4(1)$ & $C(106)$ & $\operatorname{Rh}(1)$ & $C(105)$ & $C(104)$ & 111.4(1) \\
\hline$C(106)$ & $\operatorname{Rh}(1)$ & $C(105)$ & $C(106)$ & $0.0(7)$ & $C(106)$ & $\operatorname{Rh}(1)$ & $C(105)$ & $C(115)$ & $-118.1(2)$ \\
\hline $\mathrm{C}(511)$ & $C(510)$ & $\mathrm{C}(509)$ & $\mathrm{C}(514)$ & $-3.0(2)$ & $\mathrm{C}(511)$ & $C(510)$ & $C(509)$ & $C(502)$ & $179.8(1)$ \\
\hline$C(509)$ & $C(510)$ & $\mathrm{C}(511)$ & $C(512)$ & $3.9(2)$ & $\operatorname{Rh}(3)$ & C(303) & $C(304)$ & $C(305)$ & $13.7(8)$ \\
\hline $\operatorname{Rh}(3)$ & $\mathrm{C}(303)$ & $\mathrm{C}(304)$ & $\mathrm{C}(308)$ & $129.9(9)$ & $C(302)$ & C(303) & $C(304)$ & $C(305)$ & $-59.0(1)$ \\
\hline$C(302)$ & $C(303)$ & $\mathrm{C}(304)$ & $\mathrm{C}(308)$ & $57.2(1)$ & $\operatorname{Rh}(3)$ & C(303) & $C(302)$ & $\operatorname{Rh}(3)$ & $0.00(7)$ \\
\hline $\operatorname{Rh}(3)$ & $\mathrm{C}(303)$ & $\mathrm{C}(302)$ & $\mathrm{C}(309)$ & 106.2(1) & $\operatorname{Rh}(3)$ & $C(303)$ & $C(302)$ & $C(301)$ & $-87.1(8)$ \\
\hline$C(304)$ & $C(303)$ & $\mathrm{C}(302)$ & $\operatorname{Rh}(3)$ & $87.4(9)$ & $C(304)$ & C(303) & $C(302)$ & $\mathrm{C}(309)$ & $-166.4(1)$ \\
\hline C(304) & C(303) & $\mathrm{C}(302)$ & $C(301)$ & $0.3(1)$ & $\mathrm{Rh}(1)$ & $C(102)$ & $\mathrm{C}(109)$ & C(114) & 49.9(1) \\
\hline $\operatorname{Rh}(1)$ & $\mathrm{C}(102)$ & $\mathrm{C}(109)$ & $C(110)$ & $-133.0(9)$ & $C(103)$ & $C(102)$ & $C(109)$ & $C(114)$ & $-34.4(2)$ \\
\hline$C(103)$ & $C(102)$ & $\mathrm{C}(109)$ & $C(110)$ & $142.8(1)$ & $C(101)$ & $C(102)$ & $C(109)$ & $C(114)$ & $169.7(1)$ \\
\hline$C(101)$ & $C(102)$ & $\mathrm{C}(109)$ & $C(110)$ & $-13.1(2)$ & $\mathrm{Rh}(1)$ & $C(102)$ & $C(103)$ & $\operatorname{Rh}(1)$ & $0.00(7)$ \\
\hline $\operatorname{Rh}(1)$ & $C(102)$ & $C(103)$ & $\mathrm{C}(104)$ & $88.8(8)$ & $C(109)$ & $C(102)$ & $C(103)$ & $\operatorname{Rh}(1)$ & 115.1(1) \\
\hline C(109) & $C(102)$ & $C(103)$ & $C(104)$ & $-156.1(1)$ & $C(101)$ & $C(102)$ & $C(103)$ & $\operatorname{Rh}(1)$ & $-87.0(9)$ \\
\hline$C(101)$ & $C(102)$ & $C(103)$ & $C(104)$ & $1.8(1)$ & $\operatorname{Rh}(1)$ & $C(102)$ & $C(101)$ & $C(106)$ & $-10.1(9)$ \\
\hline $\operatorname{Rh}(1)$ & $\mathrm{C}(102)$ & $\mathrm{C}(101)$ & $C(107)$ & $-126.8(9)$ & $C(109)$ & $C(102)$ & $C(101)$ & $C(106)$ & $-142.7(1)$ \\
\hline C(109) & $C(102)$ & $\mathrm{C}(101)$ & $\mathrm{C}(107)$ & $100.5(1)$ & $C(103)$ & $C(102)$ & $C(101)$ & $C(106)$ & $58.5(1)$ \\
\hline C(103) & $C(102)$ & $\mathrm{C}(101)$ & $C(107)$ & $-58.3(1)$ & $C(313)$ & C(314) & $\mathrm{C}(309)$ & $C(310)$ & $2.7(2)$ \\
\hline C(313) & $C(314)$ & $\mathrm{C}(309)$ & $\mathrm{C}(302)$ & $-177.2(1)$ & $C(309)$ & $C(314)$ & $C(313)$ & $C(312)$ & $-0.7(2)$ \\
\hline$C(603)$ & $C(604)$ & $\mathrm{C}(605)$ & $\operatorname{Rh}(6)$ & $-10.1(8)$ & $C(603)$ & $C(604)$ & $C(605)$ & $C(606)$ & $59.2(1)$ \\
\hline$C(603)$ & $C(604)$ & $\mathrm{C}(605)$ & $C(615)$ & $-134.1(1)$ & $C(608)$ & $C(604)$ & $C(605)$ & $\operatorname{Rh}(6)$ & $-127.0(9)$ \\
\hline C(608) & $C(604)$ & $\mathrm{C}(605)$ & C(606) & $-57.6(1)$ & $C(608)$ & $C(604)$ & $C(605)$ & $C(615)$ & 109.1(1) \\
\hline$C(605)$ & $C(604)$ & $\mathrm{C}(603)$ & $\operatorname{Rh}(6)$ & $10.4(9)$ & $C(605)$ & $C(604)$ & $C(603)$ & $C(602)$ & $-62.5(1)$ \\
\hline $\mathrm{C}(608)$ & C(604) & $C(603)$ & $\operatorname{Rh}(6)$ & 127.9(9) & $C(608)$ & $C(604)$ & $C(603)$ & $C(602)$ & $55.0(1)$ \\
\hline
\end{tabular}




\begin{tabular}{|c|c|c|c|c|c|c|c|}
\hline$C(605)$ & $C(604) C(608)$ & $C(607)$ & $53.9(1)$ & $C(603)$ & $C(604)$ & $C(608) C(607)$ & $-56.9(1)$ \\
\hline$C(314)$ & $\mathrm{C}(309) \mathrm{C}(310)$ & $C(311)$ & $-3.9(2)$ & $\mathrm{C}(302)$ & $\mathrm{C}(309)$ & $C(310) C(311)$ & $175.9(1)$ \\
\hline $\mathrm{C}(314)$ & $\mathrm{C}(309) \mathrm{C}(302)$ & $\operatorname{Rh}(3)$ & $70.8(1)$ & $C(314)$ & $\mathrm{C}(309)$ & $C(302) C(303)$ & $-10.0(2)$ \\
\hline$C(314)$ & $\mathrm{C}(309) \mathrm{C}(302)$ & $\mathrm{C}(301)$ & $-175.2(1)$ & $C(310)$ & $\mathrm{C}(309)$ & $\mathrm{C}(302) \mathrm{Rh}(3)$ & $-109.1(1)$ \\
\hline$C(310)$ & $\mathrm{C}(309) \mathrm{C}(302)$ & $\mathrm{C}(303)$ & $170.2(1)$ & $C(310)$ & $\mathrm{C}(309)$ & $C(302) C(301)$ & $5.0(2)$ \\
\hline $\mathrm{Rh}(4)$ & $\mathrm{C}(403) \mathrm{C}(402)$ & $\mathrm{Rh}(4)$ & $0.00(7)$ & $\operatorname{Rh}(4)$ & $\mathrm{C}(403)$ & $C(402) C(401)$ & $-87.0(7)$ \\
\hline $\mathrm{Rh}(4)$ & $\mathrm{C}(403) \mathrm{C}(402)$ & $\mathrm{C}(409)$ & 107.2(1) & $\mathrm{C}(404)$ & $\mathrm{C}(403)$ & C(402) Rh(4) & $88.0(8)$ \\
\hline$C(404)$ & $\mathrm{C}(403) \mathrm{C}(402)$ & $\mathrm{C}(401)$ & $1.0(1)$ & $\mathrm{C}(404)$ & $\mathrm{C}(403)$ & $C(402) C(409)$ & $-164.8(1)$ \\
\hline $\operatorname{Rh}(4)$ & $\mathrm{C}(403) \mathrm{C}(404)$ & $C(405)$ & $12.2(7)$ & $\operatorname{Rh}(4)$ & $\mathrm{C}(403)$ & $C(404) C(408)$ & $129.6(8)$ \\
\hline $\mathrm{C}(402)$ & $\mathrm{C}(403) \mathrm{C}(404)$ & $C(405)$ & $-60.5(1)$ & $\mathrm{C}(402)$ & $\mathrm{C}(403)$ & $C(404) C(408)$ & $57.0(1)$ \\
\hline$C(214)$ & $\mathrm{C}(213) \mathrm{C}(212)$ & $\mathrm{C}(211)$ & $-1.1(2)$ & $C(212)$ & $\mathrm{C}(213)$ & $C(214) C(209)$ & $0.1(2)$ \\
\hline $\mathrm{C}(317)$ & $C(316) C(315)$ & $C(305)$ & $-178.1(1)$ & $\mathrm{C}(317)$ & $C(316)$ & $C(315) C(320)$ & $-0.7(2)$ \\
\hline$C(315)$ & $C(316) C(317)$ & $C(318)$ & $1.8(2)$ & $C(402)$ & $\mathrm{C}(401)$ & $\mathrm{C}(406) \mathrm{Rh}(4)$ & $10.5(8)$ \\
\hline$C(402)$ & $\mathrm{C}(401) \mathrm{C}(406)$ & $C(405)$ & $-61.5(1)$ & $\mathrm{C}(407)$ & $\mathrm{C}(401)$ & $\mathrm{C}(406) \mathrm{Rh}(4)$ & $126.2(9)$ \\
\hline C(407) & $\mathrm{C}(401) \mathrm{C}(406)$ & $\mathrm{C}(405)$ & $54.2(1)$ & C(406) & $\mathrm{C}(401)$ & $\mathrm{C}(402) \mathrm{Rh}(4)$ & $-10.4(8)$ \\
\hline C(406) & $\mathrm{C}(401) \mathrm{C}(402)$ & $\mathrm{C}(403)$ & $58.9(1)$ & C(406) & $\mathrm{C}(401)$ & $C(402) C(409)$ & $-135.1(1)$ \\
\hline C(407) & $\mathrm{C}(401) \mathrm{C}(402)$ & $\mathrm{Rh}(4)$ & $-127.3(8)$ & $\mathrm{C}(407)$ & $\mathrm{C}(401)$ & $C(402) C(403)$ & $-58.0(1)$ \\
\hline C(407) & $\mathrm{C}(401) \mathrm{C}(402)$ & $\mathrm{C}(409)$ & $108.0(1)$ & C(406) & $\mathrm{C}(401)$ & C(407) C(408) & $-53.1(1)$ \\
\hline C(402) & C(401) C(407) & C(408) & $57.2(1)$ & $\mathrm{Rh}(4)$ & $C(405)$ & $\mathrm{C}(406) \mathrm{Rh}(4)$ & $0.00(7)$ \\
\hline $\operatorname{Rh}(4)$ & $\mathrm{C}(405) \mathrm{C}(406)$ & $\mathrm{C}(401)$ & $88.3(8)$ & $C(415)$ & $\mathrm{C}(405)$ & $\mathrm{C}(406) \mathrm{Rh}(4)$ & $110.3(1)$ \\
\hline$C(415)$ & $\mathrm{C}(405) \mathrm{C}(406)$ & $\mathrm{C}(401)$ & $-161.4(9)$ & C(404) & $C(405)$ & C(406) Rh(4) & $-86.7(8)$ \\
\hline C(404) & $\mathrm{C}(405) \mathrm{C}(406)$ & $\mathrm{C}(401)$ & $1.7(1)$ & $\operatorname{Rh}(4)$ & $C(405)$ & $C(415) C(416)$ & $-96.9(9)$ \\
\hline $\operatorname{Rh}(4)$ & $\mathrm{C}(405) \mathrm{C}(415)$ & $\mathrm{C}(420)$ & $83.5(1)$ & $C(406)$ & $\mathrm{C}(405)$ & $C(415) C(416)$ & 179.1(1) \\
\hline$C(406)$ & $\mathrm{C}(405) \mathrm{C}(415)$ & $\mathrm{C}(420)$ & $-0.5(2)$ & $\mathrm{C}(404)$ & $C(405)$ & $C(415) C(416)$ & $17.8(2)$ \\
\hline C(404) & $\mathrm{C}(405) \mathrm{C}(415)$ & $C(420)$ & $-161.8(1)$ & $\mathrm{Rh}(4)$ & $C(405)$ & C(404) C(403) & $-11.9(7)$ \\
\hline $\operatorname{Rh}(4)$ & $\mathrm{C}(405) \mathrm{C}(404)$ & $\mathrm{C}(408)$ & $-128.7(8)$ & $C(406)$ & $C(405)$ & C(404) C(403) & $58.2(1)$ \\
\hline$C(406)$ & $\mathrm{C}(405) \mathrm{C}(404)$ & $\mathrm{C}(408)$ & $-58.5(1)$ & $\mathrm{C}(415)$ & $\mathrm{C}(405)$ & C(404) C(403) & $-138.1(1)$ \\
\hline $\mathrm{C}(415)$ & $\mathrm{C}(405) \mathrm{C}(404)$ & $\mathrm{C}(408)$ & 105.2(1) & $\mathrm{C}(108)$ & $\mathrm{C}(104)$ & $\mathrm{C}(103) \mathrm{Rh}(1)$ & $129.5(1)$ \\
\hline $\mathrm{C}(108)$ & C(104) C(103) & $\mathrm{C}(102)$ & $55.2(2)$ & $\mathrm{C}(105)$ & $\mathrm{C}(104)$ & C(103) Rh(1) & $13.4(9)$ \\
\hline $\mathrm{C}(105)$ & C(104) C(103) & $C(102)$ & $-60.9(1)$ & $C(103)$ & $\mathrm{C}(104)$ & $C(108) C(107)$ & $-55.5(1)$ \\
\hline $\mathrm{C}(105)$ & C(104) C(108) & $C(107)$ & $55.4(1)$ & $\mathrm{C}(103)$ & $C(104)$ & $\mathrm{C}(105) \mathrm{Rh}(1)$ & $-13.0(9)$ \\
\hline $\mathrm{C}(103)$ & $C(104) C(105)$ & $C(106)$ & $56.1(1)$ & $C(103)$ & $\mathrm{C}(104)$ & $C(105) C(115)$ & $-141.2(1)$ \\
\hline $\mathrm{C}(108)$ & C(104) C(105) & $\operatorname{Rh}(1)$ & $-129.2(1)$ & $\mathrm{C}(108)$ & $\mathrm{C}(104)$ & $C(105) C(106)$ & $-60.1(1)$ \\
\hline $\mathrm{C}(108)$ & $C(104) C(105)$ & $C(115)$ & $102.7(1)$ & $C(117)$ & $\mathrm{C}(116)$ & $C(115) C(105)$ & $-177.0(1)$ \\
\hline C(117) & $C(116) C(115)$ & $C(120)$ & $-0.1(2)$ & $C(115)$ & C(116) & C(117) C(118) & $-1.2(2)$ \\
\hline$C(102)$ & $\mathrm{C}(109) \mathrm{C}(114)$ & $\mathrm{C}(113)$ & $175.9(1)$ & $C(110)$ & C(109) & $C(114) C(113)$ & $-1.3(2)$ \\
\hline$C(102)$ & $C(109) \mathrm{C}(110)$ & $\mathrm{C}(111)$ & $-175.1(1)$ & $C(114)$ & C(109) & $C(110) C(111)$ & $2.2(2)$ \\
\hline C(409) & $C(410) C(411)$ & C(412) & $-1.1(2)$ & $C(411)$ & $\mathrm{C}(410)$ & C(409) C(402) & $179.9(1)$ \\
\hline C(411) & $\mathrm{C}(410) \mathrm{C}(409)$ & C(414) & $0.5(2)$ & $C(414)$ & $\mathrm{C}(413)$ & $C(412) C(411)$ & $1.6(2)$ \\
\hline C(412) & $C(413) C(414)$ & C(409) & $-2.2(2)$ & $C(615)$ & C(616) & $C(617) C(618)$ & $1.0(2)$ \\
\hline C(617) & $C(616) C(615)$ & $C(605)$ & $178.4(1)$ & $C(617)$ & C(616) & $C(615) C(620)$ & $-2.0(2)$ \\
\hline $\operatorname{Rh}(6)$ & $C(605) \mathrm{C}(606)$ & $\mathrm{Rh}(6)$ & $0.00(7)$ & $\operatorname{Rh}(6)$ & $\mathrm{C}(605)$ & $C(606) \mathrm{C}(601)$ & $87.5(8)$ \\
\hline$C(604)$ & $C(605) \mathrm{C}(606)$ & $\operatorname{Rh}(6)$ & $-86.8(8)$ & $C(604)$ & $\mathrm{C}(605)$ & $C(606) \mathrm{C}(601)$ & $0.8(1)$ \\
\hline$C(615)$ & $C(605) C(606)$ & $\operatorname{Rh}(6)$ & $107.0(1)$ & $C(615)$ & $\mathrm{C}(605)$ & $C(606) C(601)$ & $-165.5(1)$ \\
\hline $\operatorname{Rh}(6)$ & $\mathrm{C}(605) \mathrm{C}(615)$ & $\mathrm{C}(616)$ & $-100.8(9)$ & $\operatorname{Rh}(6)$ & $\mathrm{C}(605)$ & $C(615) C(620)$ & 79.7(1) \\
\hline$C(604)$ & $\mathrm{C}(605) \mathrm{C}(615)$ & $\mathrm{C}(616)$ & $13.0(2)$ & $C(604)$ & $\mathrm{C}(605)$ & $C(615) C(620)$ & $-166.5(1)$ \\
\hline C(606) & $C(605) \mathrm{C}(615)$ & $C(616)$ & 177.9(1) & $C(606)$ & $\mathrm{C}(605)$ & $C(615) C(620)$ & $-1.7(2)$ \\
\hline C(407) & C(408) C(404) & $\mathrm{C}(403)$ & $-54.6(1)$ & $\mathrm{C}(407)$ & $\mathrm{C}(408)$ & $C(404) C(405)$ & $56.3(1)$ \\
\hline C(404) & $\mathrm{C}(408) \mathrm{C}(407)$ & $\mathrm{C}(401)$ & $-0.9(1)$ & $C(606)$ & $\mathrm{C}(601)$ & $\mathrm{C}(602) \mathrm{Rh}(6)$ & $-12.7(9)$ \\
\hline C(606) & $\mathrm{C}(601) \mathrm{C}(602)$ & $\mathrm{C}(603)$ & 56.9(1) & $C(606)$ & $\mathrm{C}(601)$ & $C(602) C(609)$ & $-136.7(1)$ \\
\hline C(607) & $\mathrm{C}(601) \mathrm{C}(602)$ & $\mathrm{Rh}(6)$ & $-128.4(1)$ & $\mathrm{C}(607)$ & $\mathrm{C}(601)$ & $C(602) C(603)$ & $-58.8(1)$ \\
\hline C(607) & $\mathrm{C}(601) \mathrm{C}(602)$ & $C(609)$ & $107.6(1)$ & $C(602)$ & $\mathrm{C}(601)$ & $\mathrm{C}(606) \mathrm{Rh}(6)$ & 12.8(9) \\
\hline$C(602)$ & $\mathrm{C}(601) \mathrm{C}(606)$ & $\mathrm{C}(605)$ & $-59.7(1)$ & $\mathrm{C}(607)$ & $\mathrm{C}(601)$ & $\mathrm{C}(606) \operatorname{Rh}(6)$ & $130.1(9)$ \\
\hline
\end{tabular}


C(607) C(601) C(606) C(605) 57.6(1) C(606) C(601) C(607) C(608) -57.7(1) Rh(3) C(306) C(305) C(304) -84.9(8) C(301) C(306) C(305) Rh(3) 90.2(9) C(301) C(306) C(305) C(315) -157.2(1) $\begin{array}{llll}\mathrm{Rh}(3) & \mathrm{C}(306) \mathrm{C}(301) & \mathrm{C}(307) & 126.0(9)\end{array}$ C(305) C(306) C(301) C(307) 52.4(1) C(418) C(417) C(416) C(415) -2.0(2) C(303) C(304) C(305) C(306) 55.1(1) C(308) C(304) C(305) $\mathrm{Rh}(3)$-129.9(1) C(308) C(304) C(305) C(315) 101.8(1) C(305) C(304) C(308) C(307) 56.7(1) C(317) C(318) C(319) C(320) 2.4(2) $\mathrm{Rh}(5) \quad \mathrm{C}(503) \mathrm{C}(502) \mathrm{Rh}(5) \quad 0.00(7)$ Rh(5) C(503) C(502) C(501) -85.8(9) C(504) C(503) C(502) C(509) -160.3(1) $\begin{array}{llll}\mathrm{Rh}(5) \quad \mathrm{C}(503) & \mathrm{C}(504) & \mathrm{C}(505) & 10.0(8)\end{array}$ C(502) C(503) C(504) C(505) -62.8(1) C(609) C(610) C(611) C(612) -1.8(2) C(611) C(610) C(609) C(614) 0.8(2) Rh(4) C(402) C(409) C(414) 86.2(1) C(403) C(402) C(409) C(414) 5.2(2) C(401) C(402) C(409) C(414) -159.0(1) C(520) C(515) C(505) C(506) 2.6(2) C(516) C(515) C(505) Rh(5) -97.8(1) C(516) C(515) C(505) C(504) 16.6(2) C(516) C(515) C(520) C(519) 3.5(2)

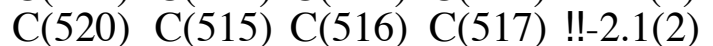
Rh(6) C(602) C(603) C(604) 89.3(8) C(601) C(602) C(603) C(604) 3.0(1) C(609) C(602) C(603) C(604) -163.2(1) $\mathrm{Rh}(6) \quad \mathrm{C}(602) \mathrm{C}(609) \mathrm{C}(614) \quad 85.4(1)$ C(601) C(602) C(609) C(614) -160.7(1) C(603) C(602) C(609) C(614) 4.0(2) C(518) C(517) C(516) C(515) 0.9(2) $\begin{array}{llll}\mathrm{C}(218) & \mathrm{C}(217) \mathrm{C}(216) & \mathrm{C}(215) & -0.7(3)\end{array}$ C(620) C(619) C(618) C(617) -0.7(2) C(417) C(418) C(419) C(420) $0.6(2)$ C(417) C(416) C(415) C(420) 2.2(2) C(208) C(207) C(201) C(206) -52.6(2) C(612) C(613) C(614) C(609) $-2.6(2)$ C(619) C(618) C(617) C(616) 0.4(2) C(510) C(509) C(514) C(513) 1.4(2) C(510) C(509) C(502) Rh(5) -84.3(1) C(510) C(509) C(502) C(501) 30.7(2) C(514) C(509) C(502) C(503) 16.2(2) C(310) C(311) C(312) C(313) -1.0(2) Rh(3) C(305) C(315) C(320) 109.7(9) C(306) C(305) C(315) C(320) 25.8(2) C(304) C(305) C(315) C(320) -135.1(1) $\mathrm{Rh}(2) \quad \mathrm{C}(206) \mathrm{C}(205) \mathrm{C}(215) \quad 105.4(1)$ C(201) C(206) C(205) Rh(2) 88.4(1) C(201) C(206) C(205) C(204) 0.9(2)
C(602) C(601) C(607) C(608) 52.9(1) $\mathrm{Rh}(3) \quad \mathrm{C}(306) \mathrm{C}(305) \mathrm{Rh}(3) \quad 0.00(7)$ Rh(3) C(306) C(305) C(315) 112.6(1) C(301) C(306) C(305) C(304) 5.4(1) $\mathrm{Rh}(3) \quad \mathrm{C}(306) \mathrm{C}(301) \mathrm{C}(302) \quad 9.5(8)$ C(305) C(306) C(301) C(302) -64.1(1) C(416) C(417) C(418) C(419) 0.5(2) C(303) C(304) C(305) Rh(3) -13.5(9) C(303) C(304) C(305) C(315) -141.7(1) C(308) C(304) C(305) C(306) -61.4(1) C(303) C(304) C(308) C(307) -54.4(1) C(109) C(114) C(113) C(112) $0.2(2)$ C(319) C(318) C(317) C(316) -2.6(2) Rh(5) C(503) C(502) C(509) $110.0(1)$ C(504) C(503) C(502) Rh(5) 89.7(1) C(504) C(503) C(502) C(501) 4.0(2) $\mathrm{Rh}(5) \quad \mathrm{C}(503) \mathrm{C}(504) \mathrm{C}(508) \quad 125.5(9)$ C(502) C(503) C(504) C(508) 52.7(2) C(611) C(610) C(609) C(602) -178.0(1) Rh(4) C(402) C(409) C(410) -93.2(1) C(403) C(402) C(409) C(410) -174.1(1) C(401) C(402) C(409) C(410) 21.7(2) C(520) C(515) C(505) Rh(5) 82.5(2) C(520) C(515) C(505) C(504) -163.0(1) C(516) C(515) C(505) C(506) -177.8(1) C(505) C(515) C(520) C(519) -176.9(1) C(505) C(515) C(516) C(517) 178.3(1) Rh(6) C(602) C(603) Rh(6) 0.00(7) C(601) C(602) C(603) Rh(6) -86.3(9) C(609) C(602) C(603) Rh(6) 107.5(1) $\mathrm{Rh}(6) \quad \mathrm{C}(602) \mathrm{C}(609) \mathrm{C}(610) \quad-95.8(1)$ C(601) C(602) C(609) C(610) 18.1(2) C(603) C(602) C(609) C(610) -177.2(1) C(410) C(411) C(412) C(413) $0.0(2)$ C(516) C(517) C(518) C(519) -0.8(2) C(216) C(217) C(218) C(219) 1.3(3) C(618) C(619) C(620) C(615) -0.3(2) C(417) C(416) C(415) C(405) -177.5(1) C(201) C(207) C(208) C(204) -1.3(2) C(208) C(207) C(201) C(202) 57.8(2) C(614) C(613) C(612) C(611) 1.7(2) C(309) C(310) C(311) C(312) 3.1(2) C(502) C(509) C(514) C(513) 178.6(1) C(510) C(509) C(502) C(503) -166.7(1) C(514) C(509) C(502) Rh(5) 98.6(1) C(514) C(509) C(502) C(501) -146.4(1) Rh(3) C(305) C(315) C(316) -72.9(1) $\mathrm{C}(306) \mathrm{C}(305) \mathrm{C}(315) \mathrm{C}(316)-156.8(1)$ C(304) C(305) C(315) C(316) 42.3(2) $\mathrm{Rh}(2) \quad \mathrm{C}(206) \mathrm{C}(205) \mathrm{Rh}(2) \quad 0.00(8)$ $\mathrm{Rh}(2) \quad \mathrm{C}(206) \mathrm{C}(205) \mathrm{C}(204)-87.6(9)$ C(201) C(206) C(205) C(215) -166.2(1) $\mathrm{Rh}(2) \quad \mathrm{C}(206) \mathrm{C}(201) \mathrm{C}(207) \quad 127.2(9)$ 


\begin{tabular}{|c|c|c|c|c|c|c|c|}
\hline $\operatorname{Rh}(2)$ & $C(206) C(201)$ & $C(202)$ & $12.1(8)$ & $C(205)$ & C(206) & $C(201) C(207)$ & $54.6(2)$ \\
\hline$C(205)$ & $C(206) C(201)$ & $\mathrm{C}(202)$ & $-60.5(1)$ & $C(102)$ & $\mathrm{C}(101)$ & $\mathrm{C}(106) \mathrm{Rh}(1)$ & $10.3(1)$ \\
\hline$C(102)$ & $C(101) C(106)$ & $C(105)$ & $-63.6(1)$ & $C(107)$ & $\mathrm{C}(101)$ & $C(106) \operatorname{Rh}(1)$ & 127.7(1) \\
\hline$C(107)$ & $C(101) C(106)$ & $C(105)$ & $53.8(2)$ & $C(102)$ & $\mathrm{C}(101)$ & $C(107) C(108)$ & $55.2(2)$ \\
\hline$C(106)$ & $C(101) C(107)$ & C(108) & $-55.5(2)$ & $C(217)$ & $C(216)$ & $C(215) C(205)$ & $177.5(1)$ \\
\hline$C(217)$ & $C(216) C(215)$ & $C(220)$ & $-0.0(2)$ & $C(112)$ & $\mathrm{C}(111)$ & $C(110) C(109)$ & $-2.0(2)$ \\
\hline$C(110)$ & $C(111) \mathrm{C}(112)$ & $C(113)$ & $0.9(2)$ & $C(217)$ & $C(218)$ & $\mathrm{C}(219) \mathrm{C}(220)$ & $-1.2(3)$ \\
\hline C(418) & $C(419) \mathrm{C}(420)$ & $C(415)$ & $-0.3(2)$ & $C(304)$ & $\mathrm{C}(308)$ & $\mathrm{C}(307) \mathrm{C}(301)$ & $-0.9(1)$ \\
\hline$C(311)$ & $C(312) C(313)$ & $C(314)$ & $-0.2(2)$ & $C(117)$ & $\mathrm{C}(118)$ & $C(119) C(120)$ & $-1.6(2)$ \\
\hline C(119) & $C(118) C(117)$ & $C(116)$ & $2.0(2)$ & $C(501)$ & $C(507)$ & $\mathrm{C}(508) \mathrm{C}(504)$ & $-1.9(2)$ \\
\hline$C(508)$ & $C(507) C(501)$ & $C(502)$ & $57.2(2)$ & $C(508)$ & $C(507)$ & $\mathrm{C}(501) \mathrm{C}(506)$ & $-53.0(2)$ \\
\hline$C(405)$ & $C(415) C(420)$ & C(419) & $178.6(1)$ & $C(416)$ & $C(415)$ & $C(420) C(419)$ & $-1.1(2)$ \\
\hline C(619) & $C(620) C(615)$ & $C(616)$ & $1.7(2)$ & $C(619)$ & $C(620)$ & $C(615) C(605)$ & $-178.8(1)$ \\
\hline $\operatorname{Rh}(5)$ & $C(505) \mathrm{C}(506)$ & $\operatorname{Rh}(5)$ & $0.00(7)$ & $\operatorname{Rh}(5)$ & $C(505)$ & $C(506) \mathrm{C}(501)$ & 88.6(9) \\
\hline$C(515)$ & $\mathrm{C}(505) \mathrm{C}(506)$ & $\operatorname{Rh}(5)$ & 106.1(1) & $C(515)$ & $C(505)$ & $C(506) \mathrm{C}(501)$ & $-165.3(1)$ \\
\hline$C(504)$ & $C(505) \mathrm{C}(506)$ & $\operatorname{Rh}(5)$ & $-87.0(9)$ & $C(504)$ & $C(505)$ & $C(506) \mathrm{C}(501)$ & $1.6(2)$ \\
\hline $\operatorname{Rh}(5)$ & $C(505) C(504)$ & $C(503)$ & $-9.8(8)$ & $\operatorname{Rh}(5)$ & $C(505)$ & $C(504) C(508)$ & $-126.9(9)$ \\
\hline$C(515)$ & $\mathrm{C}(505) \mathrm{C}(504)$ & $C(503)$ & $-133.8(1)$ & $C(515)$ & $C(505)$ & $C(504) C(508)$ & 109.0(1) \\
\hline$C(506)$ & $\mathrm{C}(505) \mathrm{C}(504)$ & $C(503)$ & 58.8(1) & $C(506)$ & $C(505)$ & $\mathrm{C}(504) \mathrm{C}(508)$ & $-58.3(1)$ \\
\hline C(209) & $C(210) C(211)$ & $C(212)$ & $1.2(2)$ & $C(211)$ & $C(210)$ & $C(209) C(202)$ & $178.0(1)$ \\
\hline$C(211)$ & $\mathrm{C}(210) \mathrm{C}(209)$ & $C(214)$ & $-2.1(2)$ & $C(104)$ & $\mathrm{C}(108)$ & $C(107) C(101)$ & $1.8(2)$ \\
\hline C(610) & $C(611) C(612)$ & $C(613)$ & $0.5(2)$ & $\operatorname{Rh}(1)$ & $C(106)$ & C(105) Rh(1) & $0.00(7)$ \\
\hline $\operatorname{Rh}(1)$ & $C(106) C(105)$ & C(104) & $-85.5(8)$ & $\operatorname{Rh}(1)$ & $C(106)$ & $C(105) C(115)$ & $112.0(1)$ \\
\hline$C(101)$ & $C(106) C(105)$ & $\operatorname{Rh}(1)$ & $90.1(1)$ & $C(101)$ & $C(106)$ & $C(105) C(104)$ & $4.6(2)$ \\
\hline $\mathrm{C}(101)$ & $C(106) C(105)$ & $C(115)$ & $-157.8(1)$ & $C(515)$ & $C(520)$ & $\mathrm{C}(519) \mathrm{C}(518)$ & $-3.6(2)$ \\
\hline C(114) & $C(113) C(112)$ & $\mathrm{C}(111)$ & $0.1(2)$ & $C(210)$ & $C(211)$ & $C(212) C(213)$ & $0.4(2)$ \\
\hline $\operatorname{Rh}(1)$ & $C(105) C(115)$ & $C(116)$ & $-114.5(9)$ & $\operatorname{Rh}(1)$ & $C(105)$ & $C(115) C(120)$ & $68.7(1)$ \\
\hline C(104) & $C(105) C(115)$ & $C(116)$ & $1.4(2)$ & $C(104)$ & $C(105)$ & $C(115) C(120)$ & $-175.4(1)$ \\
\hline C(106) & $C(105) \mathrm{C}(115)$ & $C(116)$ & 161.9(1) & $C(106)$ & $C(105)$ & $C(115) C(120)$ & $-14.9(2)$ \\
\hline$C(510)$ & $\mathrm{C}(511) \mathrm{C}(512)$ & $C(513)$ & $-3.0(2)$ & $C(413)$ & $\mathrm{C}(414)$ & $C(409) \mathrm{C}(410)$ & $1.1(2)$ \\
\hline C(413) & $\mathrm{C}(414) \mathrm{C}(409)$ & C(402) & $-178.2(1)$ & $\operatorname{Rh}(2)$ & $C(203)$ & C(202) $\operatorname{Rh}(2)$ & $0.00(8)$ \\
\hline $\operatorname{Rh}(2)$ & $C(203) C(202)$ & $\mathrm{C}(209)$ & 110.7(1) & $\operatorname{Rh}(2)$ & $C(203)$ & $C(202) C(201)$ & $-85.7(9)$ \\
\hline C(204) & $C(203) C(202)$ & $\operatorname{Rh}(2)$ & $89.6(1)$ & $C(204)$ & $C(203)$ & $C(202) C(209)$ & $-159.7(1)$ \\
\hline C(204) & $C(203) C(202)$ & $\mathrm{C}(201)$ & $3.9(2)$ & $\operatorname{Rh}(2)$ & $C(203)$ & $C(204) C(208)$ & $128.6(1)$ \\
\hline $\operatorname{Rh}(2)$ & $C(203) C(204)$ & $C(205)$ & $10.4(9)$ & $C(202)$ & $C(203)$ & $C(204) C(208)$ & $55.0(2)$ \\
\hline$C(202)$ & $C(203) C(204)$ & $C(205)$ & $-63.2(1)$ & $C(316)$ & $C(315)$ & $\mathrm{C}(320) \mathrm{C}(319)$ & $0.4(2)$ \\
\hline$C(305)$ & $\mathrm{C}(315) \mathrm{C}(320)$ & $C(319)$ & 177.9(1) & $C(207)$ & $C(208)$ & $C(204) C(203)$ & $-54.5(2)$ \\
\hline C(207) & $\mathrm{C}(208) \mathrm{C}(204)$ & $C(205)$ & $55.9(2)$ & $C(306)$ & $C(301)$ & $\mathrm{C}(302) \operatorname{Rh}(3)$ & $-9.2(9)$ \\
\hline C(306) & $\mathrm{C}(301) \mathrm{C}(302)$ & $C(303)$ & $60.2(1)$ & $C(306)$ & $\mathrm{C}(301)$ & $\mathrm{C}(302) \mathrm{C}(309)$ & $-132.8(1)$ \\
\hline$C(307)$ & $\mathrm{C}(301) \mathrm{C}(302)$ & $\operatorname{Rh}(3)$ & $-127.2(9)$ & $C(307)$ & $\mathrm{C}(301)$ & $C(302) C(303)$ & $-57.8(1)$ \\
\hline C(307) & $\mathrm{C}(301) \mathrm{C}(302)$ & $\mathrm{C}(309)$ & 109.2(1) & $C(306)$ & $\mathrm{C}(301)$ & $\mathrm{C}(307) \mathrm{C}(308)$ & $-53.6(1)$ \\
\hline C(302) & $C(301) \mathrm{C}(307)$ & $C(308)$ & $56.9(1)$ & $C(216)$ & $C(215)$ & $\mathrm{C}(205) \operatorname{Rh}(2)$ & $-86.1(2)$ \\
\hline$C(216)$ & $C(215) C(205)$ & $C(206)$ & $-167.2(1)$ & $C(216)$ & $C(215)$ & $C(205) C(204)$ & $27.4(2)$ \\
\hline$C(220)$ & $C(215) C(205)$ & $\operatorname{Rh}(2)$ & 91.3(2) & $C(220)$ & $C(215)$ & $C(205) C(206)$ & $10.3(2)$ \\
\hline$C(220)$ & $C(215) C(205)$ & $C(204)$ & $-155.1(1)$ & $C(216)$ & $C(215)$ & $\mathrm{C}(220) \mathrm{C}(219)$ & $0.1(2)$ \\
\hline$C(205)$ & $C(215) C(220)$ & $\mathrm{C}(219)$ & $-177.4(1)$ & $C(210)$ & $\mathrm{C}(209)$ & $\mathrm{C}(202) \mathrm{Rh}(2)$ & $-115.8(1)$ \\
\hline$C(210)$ & $C(209) \mathrm{C}(202)$ & $C(203)$ & 161.2(1) & $C(210)$ & $\mathrm{C}(209)$ & $C(202) C(201)$ & $-1.0(2)$ \\
\hline$C(214)$ & $C(209) C(202)$ & $\operatorname{Rh}(2)$ & $64.3(2)$ & $C(214)$ & $C(209)$ & $C(202) C(203)$ & $-18.8(2)$ \\
\hline$C(214)$ & $C(209) C(202)$ & $\mathrm{C}(201)$ & 179.1(1) & $C(210)$ & $C(209)$ & $C(214) C(213)$ & $1.4(2)$ \\
\hline$C(202)$ & C(209) & $C(213)$ & $-178.6(1)$ & $\operatorname{Rh}(2)$ & $C(202)$ & $C(201) C(207)$ & $-129.5(9)$ \\
\hline $\operatorname{Rh}(2)$ & $C(202) C(201)$ & $C(206)$ & $-11.8(8)$ & $C(203)$ & $C(202)$ & $C(201) C(207)$ & $-60.8(2)$ \\
\hline$C(203)$ & $C(202) C(201)$ & $C(206)$ & $57.0(1)$ & $C(209)$ & $C(202)$ & $C(201) C(207)$ & $103.6(1)$ \\
\hline C(209) & $C(202) C(201)$ & $C(206)$ & $-138.7(1)$ & $\operatorname{Rh}(2)$ & $C(205)$ & $C(204) C(203)$ & $-10.3(9)$ \\
\hline
\end{tabular}


$\mathrm{Rh}(2) \quad \mathrm{C}(205) \mathrm{C}(204) \mathrm{C}(208)-127.5(1)$ C(206) C(205) C(204) C(208) -57.3(2) C(215) C(205) C(204) C(208) 109.9(2) C(119) C(120) C(115) C(105) 177.4(1) C(613) C(614) C(609) C(610) 1.4(2) C(315) C(320) C(319) C(318) -1.3(2) C(601) C(607) C(608) C(604) 3.6(1) C(507) C(508) C(504) C(503) -52.3(2)

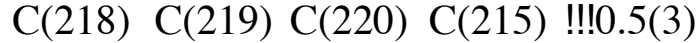

$\mathrm{Rh}(5) \quad \mathrm{C}(502) \mathrm{C}(501) \mathrm{C}(506)-12.8(8)$ C(503) C(502) C(501) C(506) 56.4(1) C(509) C(502) C(501) C(506) -139.1(1) Rh(5) C(506) C(501) C(502) 13.1(8) C(505) C(506) C(501) C(502) $-60.4(1)$

$\begin{array}{lllll}C(206) & C(205) & C(204) & C(203) & 59.9(1) \\ C(215) & C(205) & C(204) & C(203) & -132.9(1) \\ C(119) & C(120) & C(115) & C(116) & 0.5(2) \\ C(115) & C(120) & C(119) & C(118) & 0.3(2) \\ C(613) & C(614) & C(609) & C(602) & -179.8(1) \\ C(520) & C(519) & C(518) & C(517) & 2.2(2) \\ C(509) & C(514) & C(513) & C(512) & -0.7(2) \\ C(507) & C(508) & C(504) & C(505) & 57.4(2) \\ \operatorname{Rh}(5) & C(502) & C(501) & C(507) & -129.3(9) \\ C(503) & C(502) & C(501) & C(507) & -60.1(1) \\ C(509) & C(502) & C(501) & C(507) & 104.4(1) \\ \operatorname{Rh}(5) & C(506) & C(501) & C(507) & 129.0(9) \\ C(505) & C(506) & C(501) & C(507) & 55.5(1) \\ C(511) & C(512) & C(513) & C(514) & 1.4(2)\end{array}$

C(206) C(205) C(204) C(203) 59.9(1)

C(119) C(120) C(115) C(116) $0.5(2)$

C(115) C(120) C(119) C(118) 0.3(2)

C(613) C(614) C(609) C(602) -179.8(1)

C(520) C(519) C(518) C(517) 2.2(2)

C(509) C(514) C(513) C(512) -0.7(2)

C(507) C(508) C(504) C(505) 57.4(2)

C(503) C(502) C(501) C(507) -60.1(1)

C(509) C(502) C(501) C(507) 104.4(1)

C(505) C(506) C(501) C(507) 55.5(1)

C(511) C(512) C(513) C(514) 1.4(2)

Table S11. Distances beyond the asymmetric unit out to $3.60 \AA$

\begin{tabular}{|c|c|c|c|c|c|c|c|}
\hline atom & atom & distance & $\mathrm{ADC}$ & atom & atom & distance & ADC \\
\hline $\mathrm{Rh}(5)$ & $\mathrm{Cl}(6)$ & $2.40(1)$ & (1) & $\operatorname{Rh}(5)$ & $\mathrm{Cl}(5)$ & 2.41(1) & (1) \\
\hline $\operatorname{Rh}(5)$ & $C(503)$ & $2.11(3)$ & (1) & $\operatorname{Rh}(5)$ & $\mathrm{C}(515)$ & $3.1(1)$ & (1) \\
\hline $\operatorname{Rh}(5)$ & $\mathrm{C}(509)$ & $3.11(3)$ & (1) & $\operatorname{Rh}(5)$ & $\mathrm{C}(505)$ & $2.15(5)$ & (1) \\
\hline $\operatorname{Rh}(5)$ & $\mathrm{C}(502)$ & $2.13(2)$ & (1) & $\operatorname{Rh}(5)$ & $C(506)$ & $2.10(4)$ & (1) \\
\hline $\operatorname{Rh}(5)$ & $\mathrm{C}(501)$ & $2.71(7)$ & (1) & $\operatorname{Rh}(5)$ & $C(504)$ & $2.74(9)$ & (1) \\
\hline $\mathrm{Rh}(2)$ & $\mathrm{Cl}(2)$ & $2.40(1)$ & (1) & $\operatorname{Rh}(2)$ & $\mathrm{Cl}(1)$ & $2.40(3)$ & (1) \\
\hline $\operatorname{Rh}(2)$ & $C(206)$ & $2.10(4)$ & (1) & $\operatorname{Rh}(2)$ & $\mathrm{C}(203)$ & $2.09(2)$ & (1) \\
\hline $\operatorname{Rh}(2)$ & $C(215)$ & $3.05(9)$ & (1) & $\operatorname{Rh}(2)$ & C(209) & $3.12(6)$ & (1) \\
\hline $\operatorname{Rh}(2)$ & $C(202)$ & $2.14(2)$ & (1) & $\operatorname{Rh}(2)$ & $C(205)$ & $2.12(2)$ & (1) \\
\hline $\operatorname{Rh}(2)$ & $C(204)$ & $2.73(3)$ & (1) & $\operatorname{Rh}(2)$ & $\mathrm{C}(201)$ & $2.7(1)$ & (1) \\
\hline $\mathrm{Rh}(3)$ & $\mathrm{Cl}(3)$ & $2.40(1)$ & (1) & $\operatorname{Rh}(3)$ & $\mathrm{Cl}(4)$ & $2.42(8)$ & (1) \\
\hline $\operatorname{Rh}(3)$ & $C(303)$ & $2.11(2)$ & (1) & $\operatorname{Rh}(3)$ & $C(309)$ & $3.1(1)$ & (1) \\
\hline $\mathrm{Rh}(3)$ & $C(306)$ & $2.10(5)$ & (1) & $\operatorname{Rh}(3)$ & $C(304)$ & $2.70(4)$ & (1) \\
\hline $\operatorname{Rh}(3)$ & $C(305)$ & $2.14(5)$ & (1) & $\operatorname{Rh}(3)$ & $C(315)$ & $3.15(4)$ & (1) \\
\hline $\mathrm{Rh}(3)$ & $C(301)$ & $2.74(2)$ & (1) & $\operatorname{Rh}(3)$ & $\mathrm{C}(302)$ & $2.15(4)$ & (1) \\
\hline $\operatorname{Rh}(4)$ & $\mathrm{Cl}(3)$ & $2.397(7)$ & (1) & $\operatorname{Rh}(4)$ & $\mathrm{Cl}(4)$ & $2.38(2)$ & (1) \\
\hline $\operatorname{Rh}(4)$ & $\mathrm{C}(403)$ & $2.10(2)$ & (1) & $\operatorname{Rh}(4)$ & C(401) & $2.7(1)$ & (1) \\
\hline $\operatorname{Rh}(4)$ & $\mathrm{C}(405)$ & $2.13(3)$ & (1) & $\operatorname{Rh}(4)$ & C(406) & $2.12(4)$ & (1) \\
\hline $\operatorname{Rh}(4)$ & $\mathrm{C}(402)$ & $2.14(3)$ & (1) & $\operatorname{Rh}(4)$ & C(415) & 3.1(1) & (1) \\
\hline $\operatorname{Rh}(4)$ & C(404) & $2.71(3)$ & (1) & $\operatorname{Rh}(4)$ & C(409) & $3.07(3)$ & (1) \\
\hline $\operatorname{Rh}(6)$ & $\mathrm{Cl}(6)$ & $2.40(1)$ & (1) & $\operatorname{Rh}(6)$ & $\mathrm{Cl}(5)$ & $2.39(6)$ & (1) \\
\hline $\operatorname{Rh}(6)$ & $C(604)$ & $2.72(6)$ & (1) & $\operatorname{Rh}(6)$ & $C(605)$ & $2.15(2)$ & (1) \\
\hline $\operatorname{Rh}(6)$ & $C(601)$ & $2.71(8)$ & (1) & $\operatorname{Rh}(6)$ & $C(602)$ & $2.13(2)$ & (1) \\
\hline $\operatorname{Rh}(6)$ & $C(603)$ & $2.10(4)$ & (1) & $\operatorname{Rh}(6)$ & $C(606)$ & $2.11(6)$ & (1) \\
\hline $\operatorname{Rh}(6)$ & $C(615)$ & $3.09(4)$ & (1) & $\operatorname{Rh}(6)$ & $C(609)$ & $3.07(3)$ & (1) \\
\hline Rh(1) & $\mathrm{Cl}(2)$ & $2.40(2)$ & (1) & $\operatorname{Rh}(1)$ & $\mathrm{Cl}(1)$ & $2.38(5)$ & (1) \\
\hline Rh(1) & $C(102)$ & $2.17(3)$ & (1) & $\operatorname{Rh}(1)$ & $C(104)$ & $2.72(8)$ & (1) \\
\hline Rh(1) & C(109) & $3.21(7)$ & (1) & $\operatorname{Rh}(1)$ & $\mathrm{C}(103)$ & $2.10(3)$ & (1) \\
\hline Rh(1) & $C(101)$ & $2.76(2)$ & (1) & $\operatorname{Rh}(1)$ & $C(106)$ & $2.12(2)$ & (1) \\
\hline $\mathrm{Rh}(1)$ & $C(105)$ & $2.17(6)$ & (1) & $\operatorname{Rh}(1)$ & $C(115)$ & $3.17(9)$ & (1) \\
\hline $\mathrm{Cl}(2)$ & $\mathrm{Cl}(1)$ & $3.175(4)$ & (1) & $\mathrm{Cl}(2)$ & $C(410)$ & $3.50(1)$ & $(1,1,-1)$ \\
\hline $\mathrm{Cl}(2)$ & $\mathrm{C}(114)$ & $3.44(1)$ & (1) & $\mathrm{Cl}(2)$ & C(103) & $3.436(8)$ & (1) \\
\hline $\mathrm{Cl}(2)$ & $C(203)$ & $3.53(1)$ & (1) & $\mathrm{Cl}(2)$ & $C(202)$ & $3.593(9)$ & (1) \\
\hline $\mathrm{Cl}(2)$ & $\mathrm{C}(214)$ & $3.32(1)$ & (1) & $\mathrm{Cl}(3)$ & $\mathrm{Cl}(4)$ & $3.243(3)$ & (1) \\
\hline
\end{tabular}




\begin{tabular}{|c|c|c|c|c|c|c|c|}
\hline $\mathrm{Cl}(3)$ & $\mathrm{C}(604)$ & $3.596(8)$ & (1) & $\mathrm{Cl}(3)$ & $C(403)$ & $3.486(8)$ & (1) \\
\hline $\mathrm{Cl}(3)$ & $C(306)$ & $3.395(9)$ & (1) & $\mathrm{Cl}(3)$ & $\mathrm{C}(402)$ & $3.509(8)$ & (1) \\
\hline $\mathrm{Cl}(3)$ & $\mathrm{C}(305)$ & $3.576(8)$ & (1) & $\mathrm{Cl}(3)$ & $\mathrm{C}(414)$ & $3.49(1)$ & (1) \\
\hline $\mathrm{Cl}(3)$ & C(409) & $3.476(8)$ & (1) & $\mathrm{Cl}(3)$ & $\mathrm{C}(608)$ & $3.50(1)$ & (1) \\
\hline $\mathrm{Cl}(4)$ & $C(303)$ & $3.483(8)$ & (1) & $\mathrm{Cl}(4)$ & $\mathrm{C}(314)$ & $3.332(9)$ & (1) \\
\hline $\mathrm{Cl}(4)$ & $C(405)$ & $3.474(8)$ & (1) & $\mathrm{Cl}(4)$ & $\mathrm{C}(616)$ & $3.564(9)$ & (1) \\
\hline $\mathrm{Cl}(4)$ & $C(406)$ & $3.444(8)$ & (1) & $\mathrm{Cl}(4)$ & $\mathrm{C}(415)$ & $3.512(8)$ & (1) \\
\hline $\mathrm{Cl}(4)$ & $\mathrm{C}(420)$ & $3.472(9)$ & (1) & $\mathrm{Cl}(4)$ & $\mathrm{C}(617)$ & $3.50(1)$ & (1) \\
\hline $\mathrm{Cl}(6)$ & $\mathrm{Cl}(5)$ & $3.239(3)$ & (1) & $\mathrm{Cl}(6)$ & $\mathrm{C}(605)$ & $3.541(8)$ & (1) \\
\hline $\mathrm{Cl}(6)$ & $\mathrm{C}(503)$ & $3.449(9)$ & (1) & $\mathrm{Cl}(6)$ & $\mathrm{C}(509)$ & $3.575(8)$ & (1) \\
\hline $\mathrm{Cl}(6)$ & $\mathrm{C}(620)$ & $3.405(9)$ & (1) & $\mathrm{Cl}(6)$ & $\mathrm{C}(606)$ & $3.479(8)$ & (1) \\
\hline $\mathrm{Cl}(6)$ & $\mathrm{C}(502)$ & $3.530(9)$ & (1) & $\mathrm{Cl}(6)$ & $\mathrm{C}(615)$ & $3.541(8)$ & (1) \\
\hline $\mathrm{Cl}(5)$ & $\mathrm{C}(602)$ & $3.520(8)$ & (1) & $\mathrm{Cl}(5)$ & $\mathrm{C}(603)$ & $3.421(8)$ & (1) \\
\hline $\mathrm{Cl}(5)$ & $C(505)$ & $3.588(9)$ & (1) & $\mathrm{Cl}(5)$ & $\mathrm{C}(520)$ & $3.52(1)$ & (1) \\
\hline $\mathrm{Cl}(5)$ & $\mathrm{C}(614)$ & $3.49(1)$ & (1) & $\mathrm{Cl}(5)$ & $C(506)$ & $3.470(9)$ & (1) \\
\hline $\mathrm{Cl}(5)$ & $C(117)$ & $3.583(9)$ & \multicolumn{2}{|c|}{$(1,1,0,0,-1) \mathrm{Cl}(5)$} & $\mathrm{C}(609)$ & $3.531(8)$ & (1) \\
\hline $\mathrm{Cl}(1)$ & $C(206)$ & $3.401(9)$ & (1) & $\mathrm{Cl}(1)$ & $\mathrm{C}(106)$ & $3.52(1)$ & (1) \\
\hline $\mathrm{Cl}(1)$ & $\mathrm{C}(105)$ & $3.515(9)$ & (1) & $\mathrm{Cl}(1)$ & $\mathrm{C}(215)$ & $3.538(9)$ & (1) \\
\hline $\mathrm{Cl}(1)$ & $C(205)$ & $3.532(9)$ & (1) & $\mathrm{Cl}(1)$ & $\mathrm{C}(120)$ & $3.34(1)$ & (1) \\
\hline $\mathrm{Cl}(1)$ & C(319) & $3.459(9)$ & (1) & $\mathrm{Cl}(1)$ & $\mathrm{C}(115)$ & $3.571(9)$ & (1) \\
\hline $\mathrm{Cl}(1)$ & $C(220)$ & $3.56(1)$ & (1) & $\mathrm{C}(510)$ & $C(116)$ & $3.49(1)$ & $(1,1,0,0,-1)$ \\
\hline $\mathrm{C}(510)$ & $C(509)$ & $1.40(1)$ & (1) & $\mathrm{C}(510)$ & $\mathrm{C}(511)$ & $1.42(1)$ & (1) \\
\hline$C(510)$ & $C(514)$ & $2.41(2)$ & (1) & $\mathrm{C}(510)$ & $\mathrm{C}(502)$ & $2.49(1)$ & (1) \\
\hline$C(510)$ & $C(512)$ & $2.43(1)$ & (1) & $C(510)$ & $\mathrm{C}(501)$ & $3.07(1)$ & (1) \\
\hline$C(510)$ & $C(513)$ & $2.78(2)$ & (1) & $\mathrm{C}(303)$ & $\mathrm{C}(314)$ & $3.00(1)$ & (1) \\
\hline $\mathrm{C}(303)$ & C(309) & $2.56(1)$ & (1) & $\mathrm{C}(303)$ & $C(306)$ & $2.70(1)$ & (1) \\
\hline $\mathrm{C}(303)$ & $\mathrm{C}(304)$ & $1.51(1)$ & (1) & $\mathrm{C}(303)$ & $C(305)$ & $2.37(1)$ & (1) \\
\hline$C(303)$ & $\mathrm{C}(308)$ & $2.53(1)$ & (1) & $\mathrm{C}(303)$ & $\mathrm{C}(301)$ & $2.42(1)$ & (1) \\
\hline$C(303)$ & $\mathrm{C}(302)$ & $1.41(1)$ & (1) & $\mathrm{C}(303)$ & $\mathrm{C}(307)$ & $2.88(1)$ & (1) \\
\hline $\mathrm{C}(102)$ & $\mathrm{C}(104)$ & $2.46(1)$ & (1) & $\mathrm{C}(102)$ & $\mathrm{C}(109)$ & $1.48(1)$ & (1) \\
\hline $\mathrm{C}(102)$ & $\mathrm{C}(114)$ & $2.52(1)$ & (1) & $\mathrm{C}(102)$ & $\mathrm{C}(103)$ & $1.41(1)$ & (1) \\
\hline$C(102)$ & $\mathrm{C}(101)$ & $1.52(1)$ & (1) & $\mathrm{C}(102)$ & $\mathrm{C}(108)$ & $2.92(1)$ & (1) \\
\hline $\mathrm{C}(102)$ & $\mathrm{C}(106)$ & $2.34(1)$ & (1) & $\mathrm{C}(102)$ & $C(105)$ & $2.79(1)$ & (1) \\
\hline$C(102)$ & $C(110)$ & $2.48(1)$ & (1) & $\mathrm{C}(102)$ & $\mathrm{C}(107)$ & $2.50(1)$ & (1) \\
\hline$C(314)$ & C(309) & $1.38(1)$ & (1) & $\mathrm{C}(314)$ & $\mathrm{C}(413)$ & $3.50(1)$ & $(2,1,1,0,-2)$ \\
\hline C(314) & $C(310)$ & $2.38(1)$ & (1) & $\mathrm{C}(314)$ & $\mathrm{C}(311)$ & $2.77(1)$ & (1) \\
\hline$C(314)$ & $\mathrm{C}(312)$ & 2.41(1) & (1) & $\mathrm{C}(314)$ & $\mathrm{C}(302)$ & $2.49(1)$ & (1) \\
\hline$C(314)$ & $\mathrm{C}(313)$ & $1.41(1)$ & (1) & $\mathrm{C}(604)$ & $\mathrm{C}(616)$ & $3.02(1)$ & (1) \\
\hline C(604) & $C(605)$ & $1.51(1)$ & (1) & $\mathrm{C}(604)$ & $\mathrm{C}(601)$ & $2.58(1)$ & (1) \\
\hline C(604) & $\mathrm{C}(602)$ & $2.44(1)$ & (1) & $\mathrm{C}(604)$ & $\mathrm{C}(603)$ & $1.51(1)$ & (1) \\
\hline$C(604)$ & $\mathrm{C}(606)$ & $2.42(1)$ & (1) & $\mathrm{C}(604)$ & $\mathrm{C}(607)$ & $2.54(1)$ & (1) \\
\hline$C(604)$ & $\mathrm{C}(615)$ & $2.63(1)$ & (1) & $\mathrm{C}(604)$ & $\mathrm{C}(608)$ & $1.55(1)$ & (1) \\
\hline C(309) & $\mathrm{C}(310)$ & $1.40(1)$ & (1) & $\mathrm{C}(309)$ & $\mathrm{C}(311)$ & $2.44(1)$ & (1) \\
\hline C(309) & $\mathrm{C}(312)$ & 2.81(1) & (1) & $\mathrm{C}(309)$ & $\mathrm{C}(301)$ & $2.64(1)$ & (1) \\
\hline C(309) & $\mathrm{C}(302)$ & $1.48(1)$ & (1) & $\mathrm{C}(309)$ & $\mathrm{C}(313)$ & $2.43(1)$ & (1) \\
\hline C(309) & $\mathrm{C}(307)$ & $3.60(1)$ & (1) & $\mathrm{C}(403)$ & $\mathrm{C}(401)$ & $2.43(1)$ & (1) \\
\hline$C(403)$ & $\mathrm{C}(405)$ & $2.36(1)$ & (1) & $\mathrm{C}(403)$ & $\mathrm{C}(408)$ & $2.51(1)$ & (1) \\
\hline$C(403)$ & $\mathrm{C}(406)$ & $2.70(1)$ & (1) & $\mathrm{C}(403)$ & $\mathrm{C}(402)$ & $1.42(1)$ & (1) \\
\hline$C(403)$ & $\mathrm{C}(414)$ & $3.01(1)$ & (1) & $\mathrm{C}(403)$ & $\mathrm{C}(404)$ & $1.53(1)$ & (1) \\
\hline$C(403)$ & $\mathrm{C}(407)$ & $2.89(1)$ & (1) & $\mathrm{C}(403)$ & $\mathrm{C}(409)$ & $2.55(1)$ & (1) \\
\hline$C(213)$ & $\mathrm{C}(210)$ & $2.78(2)$ & (1) & $\mathrm{C}(213)$ & $\mathrm{C}(211)$ & $2.40(2)$ & (1) \\
\hline$C(213)$ & $\mathrm{C}(209)$ & $2.44(1)$ & (1) & $\mathrm{C}(213)$ & $\mathrm{C}(212)$ & $1.39(2)$ & (1) \\
\hline$C(213)$ & $\mathrm{C}(214)$ & $1.38(1)$ & (1) & $C(316)$ & $C(616)$ & $3.56(1)$ & (1) \\
\hline
\end{tabular}




\begin{tabular}{|c|c|c|c|c|c|c|c|}
\hline$C(316)$ & $C(304)$ & $3.13(1)$ & (1) & $C(316)$ & $C(318)$ & $2.41(1)$ & (1) \\
\hline$C(316)$ & $\mathrm{C}(305)$ & $2.50(1)$ & (1) & $C(316)$ & $C(315)$ & $1.40(1)$ & (1) \\
\hline$C(316)$ & $C(320)$ & $2.40(1)$ & (1) & $C(316)$ & C(319) & $2.76(1)$ & (1) \\
\hline$C(316)$ & $C(317)$ & $1.40(1)$ & (1) & $C(316)$ & $\mathrm{C}(615)$ & $3.46(1)$ & (1) \\
\hline$C(401)$ & $\mathrm{C}(405)$ & $2.46(1)$ & (1) & $\mathrm{C}(401)$ & $C(410)$ & $3.02(1)$ & (1) \\
\hline$C(401)$ & $\mathrm{C}(408)$ & $2.54(1)$ & (1) & $\mathrm{C}(401)$ & C(406) & $1.50(1)$ & (1) \\
\hline C(401) & $\mathrm{C}(402)$ & $1.53(1)$ & (1) & $\mathrm{C}(401)$ & C(404) & $2.59(1)$ & (1) \\
\hline C(401) & $\mathrm{C}(407)$ & $1.55(1)$ & (1) & $\mathrm{C}(401)$ & C(409) & $2.64(1)$ & (1) \\
\hline$C(405)$ & $\mathrm{C}(408)$ & $2.53(1)$ & (1) & $C(405)$ & C(406) & $1.42(1)$ & (1) \\
\hline$C(405)$ & $\mathrm{C}(402)$ & $2.78(1)$ & (1) & $C(405)$ & C(416) & 2.51(1) & (1) \\
\hline$C(405)$ & $\mathrm{C}(415)$ & $1.49(1)$ & (1) & $C(405)$ & C(404) & $1.53(1)$ & (1) \\
\hline$C(405)$ & $\mathrm{C}(407)$ & 2.93(1) & (1) & $C(405)$ & $C(420)$ & $2.52(1)$ & (1) \\
\hline$C(104)$ & $C(116)$ & $3.00(1)$ & (1) & C(104) & C(103) & $1.53(1)$ & (1) \\
\hline$C(104)$ & $C(101)$ & $2.57(1)$ & (1) & $C(104)$ & C(108) & $1.55(1)$ & (1) \\
\hline$C(104)$ & $C(106)$ & $2.41(1)$ & (1) & $C(104)$ & $C(105)$ & $1.51(1)$ & (1) \\
\hline$C(104)$ & $C(107)$ & $2.50(1)$ & (1) & $C(104)$ & $C(115)$ & $2.63(1)$ & (1) \\
\hline$C(116)$ & $\mathrm{C}(206)$ & $3.56(1)$ & (1) & $C(116)$ & C(118) & $2.42(1)$ & (1) \\
\hline$C(116)$ & $C(105)$ & $2.49(1)$ & (1) & $C(116)$ & $C(120)$ & $2.40(1)$ & (1) \\
\hline$C(116)$ & $C(115)$ & $1.38(1)$ & (1) & $C(116)$ & $\mathrm{C}(201)$ & $3.58(1)$ & (1) \\
\hline$C(116)$ & $\mathrm{C}(119)$ & $2.78(1)$ & (1) & $C(116)$ & C(117) & $1.40(1)$ & (1) \\
\hline $\mathrm{C}(109)$ & $C(114)$ & $1.39(1)$ & (1) & $C(109)$ & C(103) & $2.55(1)$ & (1) \\
\hline $\mathrm{C}(109)$ & $C(101)$ & 2.61(1) & (1) & $C(109)$ & $\mathrm{C}(111)$ & $2.41(1)$ & (1) \\
\hline $\mathrm{C}(109)$ & C(113) & $2.42(1)$ & (1) & $C(109)$ & $C(110)$ & $1.40(1)$ & (1) \\
\hline $\mathrm{C}(109)$ & C(107) & $3.50(1)$ & (1) & $C(109)$ & $C(112)$ & $2.81(1)$ & (1) \\
\hline$C(410)$ & $C(413)$ & $2.77(2)$ & (1) & $C(410)$ & C(402) & $2.45(1)$ & (1) \\
\hline$C(410)$ & $\mathrm{C}(411)$ & $1.41(1)$ & (1) & $C(410)$ & $\mathrm{C}(412)$ & $2.43(1)$ & (1) \\
\hline$C(410)$ & $\mathrm{C}(414)$ & $2.37(1)$ & (1) & $C(410)$ & C(409) & $1.36(1)$ & (1) \\
\hline$C(413)$ & $\mathrm{C}(411)$ & $2.36(2)$ & (1) & $C(413)$ & C(412) & $1.34(2)$ & (1) \\
\hline$C(413)$ & $\mathrm{C}(414)$ & $1.39(1)$ & (1) & $C(413)$ & C(409) & $2.43(1)$ & (1) \\
\hline$C(413)$ & $C(219)$ & $3.51(2)$ & (1) & $C(616)$ & $\mathrm{C}(605)$ & $2.51(1)$ & (1) \\
\hline$C(616)$ & $\mathrm{C}(619)$ & $2.76(1)$ & (1) & $C(616)$ & C(618) & 2.41(1) & (1) \\
\hline$C(616)$ & $C(620)$ & $2.38(1)$ & (1) & $C(616)$ & C(617) & $1.39(1)$ & (1) \\
\hline$C(616)$ & $C(615)$ & $1.40(1)$ & (1) & $C(605)$ & $\mathrm{C}(601)$ & $2.47(1)$ & (1) \\
\hline$C(605)$ & $C(602)$ & $2.77(1)$ & (1) & $C(605)$ & $C(603)$ & $2.33(1)$ & (1) \\
\hline$C(605)$ & $C(620)$ & 2.51(1) & (1) & $C(605)$ & C(606) & $1.42(1)$ & (1) \\
\hline$C(605)$ & $\mathrm{C}(607)$ & $2.92(1)$ & (1) & $C(605)$ & $\mathrm{C}(615)$ & $1.49(1)$ & (1) \\
\hline$C(605)$ & $C(608)$ & $2.53(1)$ & (1) & $\mathrm{C}(408)$ & C(406) & $2.92(1)$ & (1) \\
\hline C(408) & $\mathrm{C}(402)$ & $2.94(1)$ & (1) & C(408) & $C(217)$ & $3.46(1)$ & $(1,1,1)$ \\
\hline C(408) & $C(415)$ & $3.59(1)$ & (1) & $\mathrm{C}(408)$ & C(404) & $1.53(1)$ & (1) \\
\hline C(408) & C(407) & $1.54(1)$ & (1) & $C(601)$ & $C(610)$ & $3.06(1)$ & (1) \\
\hline $\mathrm{C}(601)$ & $C(602)$ & $1.52(1)$ & (1) & $C(601)$ & $C(603)$ & $2.43(1)$ & (1) \\
\hline$C(601)$ & $C(606)$ & $1.53(1)$ & (1) & $C(601)$ & C(607) & $1.54(1)$ & (1) \\
\hline$C(601)$ & $C(608)$ & $2.53(1)$ & (1) & $C(601)$ & C(609) & $2.64(1)$ & (1) \\
\hline$C(306)$ & $C(304)$ & $2.44(1)$ & (1) & $C(306)$ & $C(305)$ & $1.41(1)$ & (1) \\
\hline$C(306)$ & $\mathrm{C}(308)$ & $2.96(1)$ & (1) & $C(306)$ & $C(315)$ & $2.56(1)$ & (1) \\
\hline$C(306)$ & $\mathrm{C}(301)$ & $1.52(1)$ & (1) & $C(306)$ & $C(302)$ & $2.33(1)$ & (1) \\
\hline$C(306)$ & $\mathrm{C}(320)$ & $3.02(1)$ & (1) & $C(306)$ & C(307) & $2.53(1)$ & (1) \\
\hline$C(417)$ & C(418) & $1.39(1)$ & (1) & $C(417)$ & C(416) & $1.37(1)$ & (1) \\
\hline$C(417)$ & C(419) & $2.37(1)$ & (1) & $C(417)$ & $C(415)$ & $2.40(1)$ & (1) \\
\hline$C(417)$ & C(420) & $2.74(1)$ & (1) & $C(406)$ & C(402) & $2.34(1)$ & (1) \\
\hline$C(406)$ & C(415) & $2.58(1)$ & (1) & $C(406)$ & C(404) & $2.43(1)$ & (1) \\
\hline$C(406)$ & $\mathrm{C}(407)$ & $2.51(1)$ & (1) & $C(406)$ & $C(420)$ & $3.03(1)$ & (1) \\
\hline$C(304)$ & $C(305)$ & $1.54(1)$ & (1) & C(304) & C(308) & $1.57(1)$ & (1) \\
\hline
\end{tabular}




\begin{tabular}{|c|c|c|c|c|c|c|c|}
\hline$C(304)$ & $\mathrm{C}(315)$ & $2.63(1)$ & (1) & $C(304)$ & $\mathrm{C}(301)$ & $2.59(1)$ & (1) \\
\hline$C(304)$ & $\mathrm{C}(302)$ & $2.46(1)$ & (1) & $\mathrm{C}(304)$ & $\mathrm{C}(307)$ & $2.52(1)$ & (1) \\
\hline $\mathrm{C}(114)$ & $\mathrm{C}(103)$ & $3.10(1)$ & (1) & $\mathrm{C}(114)$ & $\mathrm{C}(111)$ & $2.75(2)$ & (1) \\
\hline $\mathrm{C}(114)$ & $\mathrm{C}(113)$ & $1.40(1)$ & (1) & $\mathrm{C}(114)$ & $\mathrm{C}(110)$ & $2.40(2)$ & (1) \\
\hline$C(114)$ & $\mathrm{C}(112)$ & $2.42(2)$ & (1) & $\mathrm{C}(318)$ & $C(315)$ & $2.80(1)$ & (1) \\
\hline$C(318)$ & $\mathrm{C}(320)$ & $2.41(1)$ & (1) & $\mathrm{C}(318)$ & $\mathrm{C}(319)$ & $1.39(1)$ & (1) \\
\hline $\mathrm{C}(318)$ & $\mathrm{C}(317)$ & $1.37(1)$ & (1) & $\mathrm{C}(503)$ & $\mathrm{C}(509)$ & $2.56(1)$ & (1) \\
\hline$C(503)$ & $C(507)$ & $2.94(1)$ & (1) & $\mathrm{C}(503)$ & $C(505)$ & $2.32(1)$ & (1) \\
\hline$C(503)$ & $\mathrm{C}(514)$ & $3.01(1)$ & (1) & $\mathrm{C}(503)$ & $\mathrm{C}(508)$ & $2.53(1)$ & (1) \\
\hline $\mathrm{C}(503)$ & $\mathrm{C}(502)$ & $1.43(1)$ & (1) & $\mathrm{C}(503)$ & $C(506)$ & $2.69(1)$ & (1) \\
\hline$C(503)$ & $\mathrm{C}(501)$ & $2.45(1)$ & (1) & $\mathrm{C}(503)$ & $C(504)$ & $1.52(1)$ & (1) \\
\hline $\mathrm{C}(610)$ & $C(602)$ & $2.52(1)$ & (1) & $\mathrm{C}(610)$ & $\mathrm{C}(613)$ & 2.77(1) & (1) \\
\hline $\mathrm{C}(610)$ & $C(611)$ & $1.38(1)$ & (1) & $\mathrm{C}(610)$ & $C(614)$ & $2.40(1)$ & (1) \\
\hline$C(610)$ & $C(612)$ & $2.40(1)$ & (1) & $\mathrm{C}(610)$ & $\mathrm{C}(609)$ & $1.40(1)$ & (1) \\
\hline C(402) & C(414) & $2.51(1)$ & (1) & $C(402)$ & $C(404)$ & $2.47(1)$ & (1) \\
\hline C(402) & C(407) & $2.51(1)$ & (1) & $\mathrm{C}(402)$ & $\mathrm{C}(409)$ & $1.47(1)$ & (1) \\
\hline $\mathrm{C}(515)$ & $C(517)$ & $2.41(1)$ & (1) & $\mathrm{C}(515)$ & $C(505)$ & $1.49(1)$ & (1) \\
\hline$C(515)$ & $C(520)$ & $1.41(1)$ & (1) & $C(515)$ & $C(516)$ & 1.41(1) & (1) \\
\hline$C(515)$ & $C(519)$ & $2.43(1)$ & (1) & $C(515)$ & $C(508)$ & $3.58(1)$ & (1) \\
\hline$C(515)$ & $C(506)$ & $2.59(1)$ & (1) & $\mathrm{C}(515)$ & $\mathrm{C}(518)$ & $2.83(1)$ & (1) \\
\hline$C(515)$ & $C(504)$ & $2.62(1)$ & (1) & $\mathrm{C}(602)$ & $C(603)$ & $1.40(1)$ & (1) \\
\hline $\mathrm{C}(602)$ & C(606) & $2.35(1)$ & (1) & $\mathrm{C}(602)$ & $C(614)$ & $2.52(1)$ & (1) \\
\hline$C(602)$ & $\mathrm{C}(607)$ & $2.53(1)$ & (1) & $\mathrm{C}(602)$ & $C(608)$ & 2.91(1) & (1) \\
\hline$C(602)$ & C(609) & $1.49(1)$ & (1) & $\mathrm{C}(411)$ & $\mathrm{C}(412)$ & $1.39(2)$ & (1) \\
\hline C(411) & C(414) & $2.73(2)$ & (1) & $\mathrm{C}(411)$ & $C(409)$ & $2.40(1)$ & (1) \\
\hline $\mathrm{C}(517)$ & C(416) & $3.54(1)$ & (1) & $C(517)$ & $C(520)$ & $2.73(1)$ & (1) \\
\hline $\mathrm{C}(517)$ & $C(516)$ & $1.37(1)$ & (1) & $\mathrm{C}(517)$ & $\mathrm{C}(519)$ & $2.36(1)$ & (1) \\
\hline $\mathrm{C}(517)$ & $\mathrm{C}(518)$ & $1.37(1)$ & (1) & $\mathrm{C}(217)$ & $C(216)$ & $1.38(2)$ & (1) \\
\hline $\mathrm{C}(217)$ & $\mathrm{C}(218)$ & $1.36(2)$ & (1) & $\mathrm{C}(217)$ & $\mathrm{C}(215)$ & $2.41(1)$ & (1) \\
\hline $\mathrm{C}(217)$ & C(407) & $3.55(1)$ & $(1,1,-1)$ & $\mathrm{C}(217)$ & $C(219)$ & $2.37(2)$ & (1) \\
\hline $\mathrm{C}(217)$ & $\mathrm{C}(220)$ & $2.75(2)$ & (1) & C(619) & $\mathrm{C}(618)$ & $1.39(1)$ & (1) \\
\hline $\mathrm{C}(619)$ & C(620) & $1.39(1)$ & (1) & C(619) & C(203) & $3.59(1)$ & $(2,1,0,0,-2)$ \\
\hline C(619) & $\mathrm{C}(617)$ & $2.38(1)$ & (1) & $\mathrm{C}(619)$ & $\mathrm{C}(615)$ & $2.42(1)$ & (1) \\
\hline C(418) & $C(416)$ & $2.40(1)$ & (1) & $\mathrm{C}(418)$ & $C(419)$ & $1.37(1)$ & (1) \\
\hline C (418) & $\mathrm{C}(415)$ & $2.81(1)$ & (1) & $\mathrm{C}(418)$ & $\mathrm{C}(420)$ & $2.40(1)$ & (1) \\
\hline C(416) & C(419) & $2.75(1)$ & (1) & $C(416)$ & $\mathrm{C}(415)$ & $1.39(1)$ & (1) \\
\hline C(416) & $\mathrm{C}(404)$ & $3.05(1)$ & (1) & $C(416)$ & $\mathrm{C}(516)$ & $3.58(1)$ & (1) \\
\hline C (416) & $\mathrm{C}(420)$ & $2.37(1)$ & (1) & $\mathrm{C}(207)$ & $\mathrm{C}(206)$ & $2.52(1)$ & (1) \\
\hline$C(207)$ & $\mathrm{C}(203)$ & $2.90(1)$ & (1) & $\mathrm{C}(207)$ & $\mathrm{C}(208)$ & $1.56(2)$ & (1) \\
\hline$C(207)$ & $\mathrm{C}(209)$ & $3.51(1)$ & (1) & $\mathrm{C}(207)$ & $\mathrm{C}(202)$ & $2.49(1)$ & (1) \\
\hline$C(207)$ & $\mathrm{C}(205)$ & $2.95(2)$ & (1) & $\mathrm{C}(207)$ & $C(204)$ & $2.52(2)$ & (1) \\
\hline$C(207)$ & $\mathrm{C}(201)$ & $1.54(1)$ & (1) & $\mathrm{C}(613)$ & $\mathrm{C}(611)$ & $2.38(1)$ & (1) \\
\hline$C(613)$ & $\mathrm{C}(614)$ & $1.40(1)$ & (1) & $\mathrm{C}(613)$ & $\mathrm{C}(612)$ & $1.38(1)$ & (1) \\
\hline$C(613)$ & $\mathrm{C}(609)$ & $2.44(1)$ & (1) & $\mathrm{C}(618)$ & $C(620)$ & $2.42(1)$ & (1) \\
\hline C(618) & $\mathrm{C}(617)$ & $1.37(1)$ & (1) & $\mathrm{C}(618)$ & $\mathrm{C}(615)$ & $2.82(1)$ & (1) \\
\hline$C(310)$ & $\mathrm{C}(311)$ & $1.41(1)$ & (1) & $\mathrm{C}(310)$ & $\mathrm{C}(312)$ & $2.42(1)$ & (1) \\
\hline$C(310)$ & $\mathrm{C}(301)$ & $3.03(1)$ & (1) & $\mathrm{C}(310)$ & $C(302)$ & 2.51(1) & (1) \\
\hline$C(310)$ & $\mathrm{C}(313)$ & $2.76(1)$ & (1) & $\mathrm{C}(509)$ & $C(507)$ & $3.56(1)$ & (1) \\
\hline C(509) & $\mathrm{C}(511)$ & $2.44(1)$ & (1) & $\mathrm{C}(509)$ & $C(514)$ & $1.40(1)$ & (1) \\
\hline C(509) & $C(502)$ & $1.48(1)$ & (1) & $\mathrm{C}(509)$ & $C(512)$ & $2.82(1)$ & (1) \\
\hline C(509) & $\mathrm{C}(501)$ & $2.63(1)$ & (1) & $\mathrm{C}(509)$ & $C(513)$ & $2.42(1)$ & (1) \\
\hline$C(103)$ & $\mathrm{C}(101)$ & 2.41(1) & (1) & $\mathrm{C}(103)$ & $\mathrm{C}(108)$ & $2.52(1)$ & (1) \\
\hline C(103) & $C(106)$ & $2.68(1)$ & (1) & C(103) & $C(105)$ & $2.36(1)$ & (1) \\
\hline
\end{tabular}




\begin{tabular}{|c|c|c|c|c|c|c|c|}
\hline$C(103)$ & $C(107)$ & $2.90(1)$ & (1) & $C(311)$ & $C(312)$ & $1.39(2)$ & (1) \\
\hline $\mathrm{C}(311)$ & $\mathrm{C}(313)$ & $2.38(2)$ & (1) & $C(305)$ & $\mathrm{C}(308)$ & $2.54(1)$ & (1) \\
\hline$C(305)$ & $\mathrm{C}(315)$ & $1.47(1)$ & (1) & $C(305)$ & $C(301)$ & $2.45(1)$ & (1) \\
\hline$C(305)$ & $C(302)$ & $2.78(1)$ & (1) & $C(305)$ & $C(320)$ & $2.48(1)$ & (1) \\
\hline$C(305)$ & $\mathrm{C}(307)$ & 2.91(1) & (1) & C(206) & $C(203)$ & $2.69(1)$ & (1) \\
\hline$C(206)$ & $\mathrm{C}(208)$ & 2.91(1) & (1) & $C(206)$ & $C(215)$ & $2.57(1)$ & (1) \\
\hline$C(206)$ & $C(202)$ & $2.34(1)$ & (1) & $\mathrm{C}(206)$ & $C(205)$ & $1.40(1)$ & (1) \\
\hline$C(206)$ & $C(204)$ & $2.42(1)$ & (1) & C(206) & $C(201)$ & $1.50(1)$ & (1) \\
\hline$C(206)$ & $C(220)$ & $3.02(1)$ & (1) & C(101) & $C(108)$ & $2.50(1)$ & (1) \\
\hline$C(101)$ & $C(106)$ & $1.52(1)$ & (1) & C(101) & $C(105)$ & $2.45(1)$ & (1) \\
\hline$C(101)$ & $C(110)$ & $2.96(1)$ & (1) & C(101) & $C(107)$ & $1.51(1)$ & (1) \\
\hline$C(216)$ & $\mathrm{C}(218)$ & $2.38(2)$ & (1) & $C(216)$ & $C(312)$ & $3.58(1)$ & $(2,1,1,-1,-2)$ \\
\hline$C(216)$ & $C(215)$ & $1.41(1)$ & (1) & $C(216)$ & $\mathrm{C}(407)$ & $3.56(1)$ & $(1,1,-1)$ \\
\hline$C(216)$ & $C(205)$ & $2.53(1)$ & (1) & $C(216)$ & $C(204)$ & $3.13(2)$ & (1) \\
\hline$C(216)$ & $C(219)$ & $2.76(2)$ & (1) & $C(216)$ & $\mathrm{C}(220)$ & $2.40(2)$ & (1) \\
\hline$C(111)$ & $\mathrm{C}(113)$ & $2.35(2)$ & (1) & C(111) & $C(110)$ & $1.39(1)$ & (1) \\
\hline $\mathrm{C}(111)$ & $C(112)$ & $1.36(2)$ & (1) & $\mathrm{C}(218)$ & $C(215)$ & $2.76(2)$ & (1) \\
\hline$C(218)$ & $C(219)$ & $1.37(2)$ & (1) & $C(218)$ & $C(220)$ & $2.38(2)$ & (1) \\
\hline C(419) & $C(415)$ & $2.42(1)$ & (1) & C(419) & $C(420)$ & $1.39(1)$ & (1) \\
\hline C(308) & $\mathrm{C}(315)$ & $3.54(1)$ & (1) & C(308) & $C(301)$ & $2.55(1)$ & (1) \\
\hline C(308) & $\mathrm{C}(302)$ & $2.96(1)$ & (1) & C(308) & $C(307)$ & $1.55(1)$ & (1) \\
\hline$C(312)$ & $C(313)$ & $1.36(1)$ & (1) & C(118) & $C(120)$ & $2.39(1)$ & (1) \\
\hline $\mathrm{C}(118)$ & $\mathrm{C}(115)$ & 2.81(1) & (1) & C(118) & $C(119)$ & $1.40(1)$ & (1) \\
\hline $\mathrm{C}(118)$ & C(117) & $1.40(1)$ & (1) & $C(603)$ & $C(606)$ & $2.70(1)$ & (1) \\
\hline$C(603)$ & C(614) & $2.99(1)$ & (1) & $C(603)$ & $C(607)$ & $2.93(1)$ & (1) \\
\hline$C(603)$ & $\mathrm{C}(608)$ & $2.52(1)$ & (1) & $\mathrm{C}(603)$ & $C(609)$ & $2.55(1)$ & (1) \\
\hline $\mathrm{C}(507)$ & $\mathrm{C}(505)$ & 2.94(1) & (1) & $C(507)$ & $C(508)$ & $1.55(2)$ & (1) \\
\hline $\mathrm{C}(507)$ & $\mathrm{C}(502)$ & $2.52(1)$ & (1) & $C(507)$ & $C(506)$ & $2.52(1)$ & (1) \\
\hline$C(507)$ & $C(501)$ & $1.55(1)$ & (1) & $C(507)$ & $C(504)$ & $2.55(1)$ & (1) \\
\hline C(415) & C(404) & $2.65(1)$ & (1) & C(415) & $C(420)$ & $1.39(1)$ & (1) \\
\hline$C(620)$ & $C(606)$ & $3.02(1)$ & (1) & $C(620)$ & $C(617)$ & $2.75(1)$ & (1) \\
\hline$C(620)$ & $C(615)$ & $1.39(1)$ & (1) & $C(606)$ & $C(607)$ & $2.50(1)$ & (1) \\
\hline$C(606)$ & $C(615)$ & $2.58(1)$ & (1) & $C(606)$ & $C(608)$ & $2.92(1)$ & (1) \\
\hline$C(505)$ & $\mathrm{C}(520)$ & $2.53(1)$ & (1) & $\mathrm{C}(505)$ & $C(516)$ & $2.54(1)$ & (1) \\
\hline$C(505)$ & $\mathrm{C}(508)$ & $2.49(1)$ & (1) & $C(505)$ & $C(502)$ & $2.77(1)$ & (1) \\
\hline$C(505)$ & $C(506)$ & $1.43(1)$ & (1) & $C(505)$ & $\mathrm{C}(501)$ & $2.47(1)$ & (1) \\
\hline$C(505)$ & $C(504)$ & $1.51(1)$ & (1) & $C(210)$ & $C(211)$ & $1.38(1)$ & (1) \\
\hline$C(210)$ & $\mathrm{C}(209)$ & $1.40(1)$ & (1) & $C(210)$ & $C(202)$ & $2.52(1)$ & (1) \\
\hline$C(210)$ & $C(201)$ & $3.02(1)$ & (1) & $C(210)$ & $C(212)$ & 2.41(1) & (1) \\
\hline$C(210)$ & $C(214)$ & $2.40(2)$ & (1) & C(108) & $C(611)$ & $3.51(1)$ & $(2,1,0,0,-2)$ \\
\hline C(108) & $C(106)$ & $2.90(1)$ & (1) & C(108) & $C(105)$ & $2.50(1)$ & (1) \\
\hline $\mathrm{C}(108)$ & $\mathrm{C}(107)$ & $1.52(1)$ & (1) & C(108) & $C(115)$ & $3.54(1)$ & (1) \\
\hline C(412) & $\mathrm{C}(414)$ & $2.36(1)$ & (1) & C(412) & C(409) & $2.79(1)$ & (1) \\
\hline$C(611)$ & $C(614)$ & $2.75(1)$ & (1) & $\mathrm{C}(611)$ & $C(612)$ & $1.37(1)$ & (1) \\
\hline$C(611)$ & $\mathrm{C}(609)$ & $2.42(1)$ & (1) & C(106) & $C(105)$ & $1.41(1)$ & (1) \\
\hline$C(106)$ & $\mathrm{C}(120)$ & $3.03(1)$ & (1) & C(106) & $C(107)$ & $2.49(1)$ & (1) \\
\hline$C(106)$ & $C(115)$ & $2.55(1)$ & (1) & $C(520)$ & $C(516)$ & $2.40(1)$ & (1) \\
\hline$C(520)$ & $\mathrm{C}(519)$ & $1.38(1)$ & (1) & $C(520)$ & $C(506)$ & $3.04(1)$ & (1) \\
\hline$C(520)$ & $\mathrm{C}(518)$ & 2.41(1) & (1) & C(113) & $C(110)$ & $2.75(2)$ & (1) \\
\hline$C(113)$ & C(112) & $1.38(2)$ & (1) & $C(211)$ & $C(209)$ & $2.42(1)$ & (1) \\
\hline$C(211)$ & $C(212)$ & $1.40(2)$ & (1) & $C(211)$ & $C(214)$ & $2.76(2)$ & (1) \\
\hline$C(105)$ & $\mathrm{C}(120)$ & $2.53(1)$ & (1) & $C(105)$ & $C(107)$ & $2.88(1)$ & (1) \\
\hline$C(105)$ & $\mathrm{C}(115)$ & $1.48(1)$ & (1) & $C(511)$ & $C(514)$ & $2.78(2)$ & (1) \\
\hline
\end{tabular}




\begin{tabular}{|c|c|c|c|c|c|c|c|}
\hline$C(511)$ & $C(512)$ & $1.39(2)$ & (1) & $C(511)$ & $C(513)$ & $2.40(2)$ & (1) \\
\hline$C(414)$ & C(409) & $1.40(1)$ & (1) & $C(203)$ & $\mathrm{C}(208)$ & $2.50(1)$ & (1) \\
\hline$C(203)$ & C(209) & $2.56(1)$ & (1) & $\mathrm{C}(203)$ & $C(202)$ & $1.41(1)$ & (1) \\
\hline$C(203)$ & $C(205)$ & $2.35(1)$ & (1) & $\mathrm{C}(203)$ & $C(204)$ & $1.52(1)$ & (1) \\
\hline$C(203)$ & $C(201)$ & $2.43(1)$ & (1) & $\mathrm{C}(203)$ & $C(214)$ & $3.03(1)$ & (1) \\
\hline$C(315)$ & $C(320)$ & $1.39(1)$ & (1) & $C(315)$ & C(319) & $2.40(1)$ & (1) \\
\hline$C(315)$ & $C(317)$ & $2.42(1)$ & (1) & $\mathrm{C}(208)$ & $\mathrm{C}(202)$ & $2.90(1)$ & (1) \\
\hline$C(208)$ & $C(205)$ & $2.54(1)$ & (1) & $\mathrm{C}(208)$ & $\mathrm{C}(204)$ & $1.51(2)$ & (1) \\
\hline$C(208)$ & $C(317)$ & $3.52(1)$ & $(2,1,0,-1,-2)$ & C(208) & $\mathrm{C}(201)$ & $2.53(1)$ & (1) \\
\hline$C(301)$ & $C(302)$ & $1.52(1)$ & (1) & $\mathrm{C}(301)$ & C(307) & $1.53(1)$ & (1) \\
\hline$C(215)$ & $\mathrm{C}(205)$ & $1.50(1)$ & (1) & $C(215)$ & $\mathrm{C}(204)$ & $2.69(2)$ & (1) \\
\hline$C(215)$ & $\mathrm{C}(219)$ & $2.39(2)$ & (1) & $\mathrm{C}(215)$ & $\mathrm{C}(220)$ & $1.37(2)$ & (1) \\
\hline C(209) & $\mathrm{C}(202)$ & $1.47(1)$ & (1) & $\mathrm{C}(209)$ & $\mathrm{C}(201)$ & $2.62(1)$ & (1) \\
\hline C(209) & $\mathrm{C}(212)$ & 2.81(1) & (1) & $\mathrm{C}(209)$ & $\mathrm{C}(214)$ & $1.42(1)$ & (1) \\
\hline C(404) & $\mathrm{C}(407)$ & $2.51(1)$ & (1) & $\mathrm{C}(407)$ & $\mathrm{C}(409)$ & $3.59(1)$ & (1) \\
\hline$C(302)$ & $\mathrm{C}(307)$ & $2.50(1)$ & (1) & $\mathrm{C}(516)$ & $\mathrm{C}(519)$ & $2.76(1)$ & (1) \\
\hline$C(516)$ & $\mathrm{C}(518)$ & $2.40(1)$ & (1) & C(516) & $C(504)$ & $3.06(1)$ & (1) \\
\hline$C(202)$ & $C(205)$ & $2.77(1)$ & (1) & $\mathrm{C}(202)$ & C(204) & $2.45(1)$ & (1) \\
\hline$C(202)$ & $\mathrm{C}(201)$ & $1.53(1)$ & (1) & $\mathrm{C}(202)$ & $C(214)$ & $2.51(1)$ & (1) \\
\hline$C(205)$ & $\mathrm{C}(204)$ & $1.55(1)$ & (1) & $C(205)$ & $\mathrm{C}(201)$ & $2.45(1)$ & (1) \\
\hline$C(205)$ & $C(220)$ & $2.50(1)$ & (1) & $\mathrm{C}(120)$ & $C(115)$ & $1.42(1)$ & (1) \\
\hline$C(120)$ & C(119) & $1.36(1)$ & (1) & $\mathrm{C}(120)$ & C(117) & $2.78(1)$ & (1) \\
\hline C(614) & $\mathrm{C}(612)$ & 2.41(1) & (1) & $\mathrm{C}(614)$ & $C(609)$ & 1.41(1) & (1) \\
\hline$C(320)$ & $C(319)$ & $1.37(1)$ & (1) & $\mathrm{C}(320)$ & $C(317)$ & $2.77(1)$ & (1) \\
\hline$C(319)$ & $C(317)$ & $2.38(1)$ & (1) & $C(319)$ & $C(219)$ & $3.50(2)$ & (1) \\
\hline$C(204)$ & $\mathrm{C}(201)$ & $2.58(1)$ & (1) & C(110) & $C(112)$ & $2.40(2)$ & (1) \\
\hline$C(612)$ & C(609) & 2.81(1) & (1) & C(617) & $C(615)$ & $2.42(1)$ & (1) \\
\hline$C(112)$ & C(307) & $3.54(1)$ & $(1,1,-1)$ & $\mathrm{C}(519)$ & $C(514)$ & $3.43(2)$ & $(2,1,1,-1,-3)$ \\
\hline $\mathrm{C}(519)$ & $\mathrm{C}(518)$ & $1.40(1)$ & (1) & $\mathrm{C}(607)$ & $C(608)$ & $1.56(1)$ & (1) \\
\hline$C(115)$ & C(119) & $2.43(1)$ & (1) & C(115) & C(117) & $2.42(1)$ & (1) \\
\hline$C(514)$ & $\mathrm{C}(502)$ & $2.51(1)$ & (1) & $\mathrm{C}(514)$ & $C(512)$ & $2.42(2)$ & (1) \\
\hline$C(514)$ & $\mathrm{C}(513)$ & $1.38(1)$ & (1) & $\mathrm{C}(508)$ & $\mathrm{C}(502)$ & $2.92(1)$ & (1) \\
\hline$C(508)$ & $\mathrm{C}(506)$ & $2.89(1)$ & (1) & C(508) & $C(501)$ & $2.53(1)$ & (1) \\
\hline $\mathrm{C}(508)$ & $\mathrm{C}(504)$ & $1.55(1)$ & (1) & $\mathrm{C}(219)$ & $\mathrm{C}(220)$ & $1.38(1)$ & (1) \\
\hline$C(502)$ & C(506) & $2.35(1)$ & (1) & $\mathrm{C}(502)$ & $\mathrm{C}(501)$ & $1.53(1)$ & (1) \\
\hline$C(502)$ & $C(504)$ & $2.46(1)$ & (1) & C(119) & $\mathrm{C}(117)$ & $2.42(1)$ & (1) \\
\hline$C(506)$ & $\mathrm{C}(501)$ & $1.52(1)$ & (1) & $C(506)$ & $C(504)$ & $2.43(1)$ & (1) \\
\hline$C(212)$ & $\mathrm{C}(214)$ & $2.39(2)$ & (1) & $C(512)$ & $C(513)$ & $1.40(2)$ & (1) \\
\hline $\mathrm{C}(501)$ & $\mathrm{C}(504)$ & $2.61(1)$ & (1) & & & & \\
\hline
\end{tabular}

*ADC (S, L, TX, TY, TZ) represents:

S: Symmetry operation number. $-\mathrm{S}$ is inversion of operation $\mathrm{S}$.

(1) $X, Y, Z$

(2) $-X, Y+1 / 2,-Z$

L: Lattice translation.

(1) $0,0,0$

TX, TY, TZ: Unit cell translation along the $\mathrm{x}, \mathrm{y}$, and $\mathrm{z}$ directions.

Table S12. Distances beyond the asymmetric unit out to $3.60 \AA$ 


\begin{tabular}{|c|c|c|c|c|c|c|c|}
\hline atom & atom & distance & $\mathrm{ADC}$ & atom & atom & distance & $\mathrm{ADC}$ \\
\hline $\operatorname{Rh}(5)$ & $\mathrm{Cl}(6)$ & $2.40(1)$ & (1) & $\operatorname{Rh}(5)$ & $\mathrm{Cl}(5)$ & $2.41(1)$ & (1) \\
\hline $\operatorname{Rh}(5)$ & $C(503)$ & $2.11(3)$ & (1) & $\operatorname{Rh}(5)$ & $\mathrm{C}(515)$ & $3.1(1)$ & (1) \\
\hline $\operatorname{Rh}(5)$ & $C(509)$ & $3.11(3)$ & (1) & $\operatorname{Rh}(5)$ & $\mathrm{C}(505)$ & $2.15(5)$ & (1) \\
\hline $\operatorname{Rh}(5)$ & $C(502)$ & $2.13(2)$ & (1) & $\operatorname{Rh}(5)$ & $C(506)$ & $2.10(4)$ & (1) \\
\hline $\operatorname{Rh}(5)$ & $\mathrm{C}(501)$ & $2.71(7)$ & (1) & $\operatorname{Rh}(5)$ & $C(504)$ & 2.74(9) & (1) \\
\hline $\operatorname{Rh}(2)$ & $\mathrm{Cl}(2)$ & $2.40(1)$ & (1) & $\operatorname{Rh}(2)$ & $\mathrm{Cl}(1)$ & $2.40(3)$ & (1) \\
\hline $\operatorname{Rh}(2)$ & $C(206)$ & $2.10(4)$ & (1) & $\operatorname{Rh}(2)$ & $C(203)$ & $2.09(2)$ & (1) \\
\hline $\operatorname{Rh}(2)$ & $C(215)$ & $3.05(9)$ & (1) & $\operatorname{Rh}(2)$ & $\mathrm{C}(209)$ & $3.12(6)$ & (1) \\
\hline $\operatorname{Rh}(2)$ & $C(202)$ & $2.14(2)$ & (1) & $\operatorname{Rh}(2)$ & $C(205)$ & $2.12(2)$ & (1) \\
\hline $\operatorname{Rh}(2)$ & C(204) & $2.73(3)$ & (1) & $\operatorname{Rh}(2)$ & $\mathrm{C}(201)$ & 2.7(1) & (1) \\
\hline $\operatorname{Rh}(3)$ & $\mathrm{Cl}(3)$ & $2.40(1)$ & (1) & $\operatorname{Rh}(3)$ & $\mathrm{Cl}(4)$ & $2.42(8)$ & (1) \\
\hline $\operatorname{Rh}(3)$ & $\mathrm{C}(303)$ & $2.11(2)$ & (1) & $\operatorname{Rh}(3)$ & $C(309)$ & 3.1(1) & (1) \\
\hline $\operatorname{Rh}(3)$ & $\mathrm{C}(306)$ & $2.10(5)$ & (1) & $\operatorname{Rh}(3)$ & $C(304)$ & $2.70(4)$ & (1) \\
\hline $\operatorname{Rh}(3)$ & $\mathrm{C}(305)$ & $2.14(5)$ & (1) & $\operatorname{Rh}(3)$ & $C(315)$ & $3.15(4)$ & (1) \\
\hline $\operatorname{Rh}(3)$ & $\mathrm{C}(301)$ & $2.74(2)$ & (1) & $\operatorname{Rh}(3)$ & $C(302)$ & $2.15(4)$ & (1) \\
\hline $\operatorname{Rh}(4)$ & $\mathrm{Cl}(3)$ & $2.397(7)$ & (1) & $\operatorname{Rh}(4)$ & $\mathrm{Cl}(4)$ & $2.38(2)$ & (1) \\
\hline $\operatorname{Rh}(4)$ & C(403) & $2.10(2)$ & (1) & $\operatorname{Rh}(4)$ & $C(401)$ & 2.7(1) & (1) \\
\hline $\operatorname{Rh}(4)$ & C(405) & $2.13(3)$ & (1) & $\operatorname{Rh}(4)$ & $C(406)$ & $2.12(4)$ & (1) \\
\hline $\operatorname{Rh}(4)$ & $\mathrm{C}(402)$ & $2.14(3)$ & (1) & $\operatorname{Rh}(4)$ & $C(415)$ & $3.1(1)$ & (1) \\
\hline $\operatorname{Rh}(4)$ & C(404) & 2.71(3) & (1) & $\operatorname{Rh}(4)$ & $C(409)$ & $3.07(3)$ & (1) \\
\hline $\operatorname{Rh}(6)$ & $\mathrm{Cl}(6)$ & $2.40(1)$ & (1) & $\operatorname{Rh}(6)$ & $\mathrm{Cl}(5)$ & $2.39(6)$ & (1) \\
\hline $\operatorname{Rh}(6)$ & $\mathrm{C}(604)$ & $2.72(6)$ & (1) & $\operatorname{Rh}(6)$ & $C(605)$ & $2.15(2)$ & (1) \\
\hline $\operatorname{Rh}(6)$ & $\mathrm{C}(601)$ & $2.71(8)$ & (1) & $\operatorname{Rh}(6)$ & $C(602)$ & $2.13(2)$ & (1) \\
\hline $\operatorname{Rh}(6)$ & C(603) & $2.10(4)$ & (1) & $\operatorname{Rh}(6)$ & $C(606)$ & 2.11(6) & (1) \\
\hline $\operatorname{Rh}(6)$ & $\mathrm{C}(615)$ & $3.09(4)$ & (1) & $\operatorname{Rh}(6)$ & $C(609)$ & $3.07(3)$ & (1) \\
\hline $\operatorname{Rh}(1)$ & $\mathrm{Cl}(2)$ & $2.40(2)$ & (1) & $\operatorname{Rh}(1)$ & $\mathrm{Cl}(1)$ & $2.38(5)$ & (1) \\
\hline $\mathrm{Rh}(1)$ & $C(102)$ & $2.17(3)$ & (1) & $\operatorname{Rh}(1)$ & C(104) & $2.72(8)$ & (1) \\
\hline $\operatorname{Rh}(1)$ & C(109) & $3.21(7)$ & (1) & $\operatorname{Rh}(1)$ & $C(103)$ & $2.10(3)$ & (1) \\
\hline $\operatorname{Rh}(1)$ & $\mathrm{C}(101)$ & $2.76(2)$ & (1) & $\operatorname{Rh}(1)$ & C(106) & $2.12(2)$ & (1) \\
\hline $\mathrm{Rh}(1)$ & $C(105)$ & $2.17(6)$ & (1) & $\operatorname{Rh}(1)$ & $C(115)$ & $3.17(9)$ & (1) \\
\hline $\mathrm{Cl}(2)$ & $\mathrm{H}(409)$ & $3.0(7)$ & $(1,1,-1)$ & $\mathrm{Cl}(2)$ & $\mathrm{H}(401)$ & $3.3(1)$ & $(1,1,-1)$ \\
\hline $\mathrm{Cl}(2)$ & $\mathrm{H}(213)$ & $2.91(4)$ & (1) & $\mathrm{Cl}(2)$ & $\mathrm{H}(202)$ & $3.51(4)$ & (1) \\
\hline $\mathrm{Cl}(2)$ & $\mathrm{H}(113)$ & $2.69(6)$ & (1) & $\mathrm{Cl}(2)$ & $\mathrm{H}(102)$ & $3.36(7)$ & (1) \\
\hline $\mathrm{Cl}(3)$ & $\mathrm{H}(515)$ & $3.3(1)$ & (1) & $\mathrm{Cl}(3)$ & $\mathrm{H}(614)$ & $3.25(6)$ & (1) \\
\hline $\mathrm{Cl}(3)$ & $\mathrm{H}(608)$ & $3.3(1)$ & (1) & $\mathrm{Cl}(3)$ & $\mathrm{H}(607)$ & $3.2(1)$ & (1) \\
\hline $\mathrm{Cl}(3)$ & $\mathrm{H}(603)$ & 2.9(1) & (1) & $\mathrm{Cl}(3)$ & $\mathrm{H}(413)$ & $3.47(6)$ & (1) \\
\hline $\mathrm{Cl}(3)$ & $\mathrm{H}(304)$ & $3.32(6)$ & (1) & $\mathrm{Cl}(3)$ & $\mathrm{H}(402)$ & $3.46(3)$ & (1) \\
\hline $\mathrm{Cl}(4)$ & $\mathrm{H}(514)$ & $3.15(6)$ & (1) & $\mathrm{Cl}(4)$ & $\mathrm{H}(614)$ & $3.0(1)$ & (1) \\
\hline $\mathrm{Cl}(4)$ & $H(615)$ & $2.82(7)$ & (1) & $\mathrm{Cl}(4)$ & $\mathrm{H}(313)$ & $3.01(5)$ & (1) \\
\hline $\mathrm{Cl}(4)$ & $\mathrm{H}(418)$ & $3.30(3)$ & (1) & $\mathrm{Cl}(4)$ & $\mathrm{H}(302)$ & $3.43(6)$ & (1) \\
\hline $\mathrm{Cl}(4)$ & $\mathrm{H}(404)$ & $3.4(1)$ & (1) & $\mathrm{Cl}(6)$ & $\mathrm{H}(610)$ & $3.55(8)$ & $(2,1,0,0,-3)$ \\
\hline $\mathrm{Cl}(6)$ & $\mathrm{H}(618)$ & $3.22(8)$ & (1) & $\mathrm{Cl}(6)$ & $\mathrm{H}(604)$ & $3.4(1)$ & (1) \\
\hline $\mathrm{Cl}(6)$ & $\mathrm{H}(502)$ & $3.4(1)$ & (1) & $\mathrm{Cl}(6)$ & $\mathrm{H}(114)$ & $3.0(2)$ & $(1,1,0,0,-1)$ \\
\hline $\mathrm{Cl}(6)$ & $\mathrm{H}(103)$ & $3.3(1)$ & $(1,1,0,0$ & & $\mathrm{H}(107)$ & $3.3(6)$ & $(1,1,0,0,-1)$ \\
\hline $\mathrm{Cl}(5)$ & $\mathrm{H}(518)$ & $3.40(5)$ & (1) & $\mathrm{Cl}(5)$ & $\mathrm{H}(613)$ & $3.37(3)$ & (1) \\
\hline $\mathrm{Cl}(5)$ & $\mathrm{H}(602)$ & $3.36(5)$ & (1) & $\mathrm{Cl}(5)$ & $\mathrm{H}(504)$ & $3.42(8)$ & (1) \\
\hline $\mathrm{Cl}(5)$ & H(209) & $3.5(2)$ & $(1,1,0,0$ & & $\mathrm{H}(201)$ & $3.6(1)$ & $(1,1,0,0,-1)$ \\
\hline $\mathrm{Cl}(5)$ & $\mathrm{H}(114)$ & $3.3(7)$ & $(1,1,0,0$ & & $\mathrm{H}(115)$ & $2.8(3)$ & $(1,1,0,0,-1)$ \\
\hline $\mathrm{Cl}(1)$ & $\mathrm{H}(310)$ & $3.3(2)$ & $(1,1,-1)$ & $\mathrm{Cl}(1)$ & $\mathrm{H}(318)$ & $3.6(2)$ & (1) \\
\hline $\mathrm{Cl}(1)$ & $\mathrm{H}(317)$ & $2.78(7)$ & (1) & $\mathrm{Cl}(1)$ & $\mathrm{H}(405)$ & $3.1(9)$ & $(1,1,-1)$ \\
\hline $\mathrm{Cl}(1)$ & $\mathrm{H}(218)$ & $3.51(4)$ & (1) & $\mathrm{Cl}(1)$ & $\mathrm{H}(204)$ & $3.3(1)$ & (1) \\
\hline
\end{tabular}




\begin{tabular}{|c|c|c|c|c|c|c|c|}
\hline $\mathrm{Cl}(1)$ & $\mathrm{H}(118)$ & $2.99(6)$ & (1) & $\mathrm{Cl}(1)$ & $\mathrm{H}(104)$ & $3.50(4)$ & (1) \\
\hline $\mathrm{C}(510)$ & $\mathrm{H}(511)$ & $3.29(5)$ & (1) & $\mathrm{C}(510)$ & $\mathrm{H}(509)$ & $0.95(1)$ & (1) \\
\hline $\mathrm{C}(510)$ & $\mathrm{H}(510)$ & $2.06(6)$ & (1) & $\mathrm{C}(510)$ & $\mathrm{H}(513)$ & $3.27(5)$ & (1) \\
\hline $\mathrm{C}(510)$ & $\mathrm{H}(516)$ & $3.4(5)$ & \multicolumn{2}{|c|}{$(2,1,1,0,-3) C(510)$} & $\mathrm{H}(501)$ & $2.70(5)$ & (1) \\
\hline $\mathrm{C}(510)$ & $\mathrm{H}(112)$ & $3.6(5)$ & \multirow{2}{*}{\multicolumn{2}{|c|}{$\begin{array}{l}(1,1,1,0,-1) C(510) \\
(1,1,0,0,-1) C(303)\end{array}$}} & $\mathrm{H}(111)$ & $3.1(5)$ & $(1,1,1,0,-1)$ \\
\hline $\mathrm{C}(510)$ & $\mathrm{H}(107)$ & $3.4(2)$ & & & $\mathrm{H}(615)$ & $3.60(6)$ & (1) \\
\hline $\mathrm{C}(303)$ & $\mathrm{H}(313)$ & $2.67(6)$ & (1) & $\mathrm{C}(303)$ & $\mathrm{H}(308)$ & $2.75(7)$ & (1) \\
\hline $\mathrm{C}(303)$ & $\mathrm{H}(307)$ & $3.3(1)$ & (1) & $\mathrm{C}(303)$ & $\mathrm{H}(306)$ & $3.19(4)$ & (1) \\
\hline $\mathrm{C}(303)$ & $\mathrm{H}(301)$ & $3.24(7)$ & (1) & $C(303)$ & $\mathrm{H}(303)$ & $2.06(3)$ & (1) \\
\hline$C(303)$ & $\mathrm{H}(302)$ & $0.95(1)$ & (1) & $C(102)$ & $\mathrm{H}(310)$ & $3.4(1)$ & $(1,1,-1)$ \\
\hline$C(102)$ & H(113) & $2.69(3)$ & (1) & $C(102)$ & H(109) & $2.64(4)$ & (1) \\
\hline $\mathrm{C}(102)$ & $\mathrm{H}(103)$ & $3.3(1)$ & (1) & $\mathrm{C}(102)$ & $\mathrm{H}(101)$ & $2.07(3)$ & (1) \\
\hline$C(102)$ & $\mathrm{H}(108)$ & $3.2(1)$ & (1) & $C(102)$ & $\mathrm{H}(105)$ & $3.31(7)$ & (1) \\
\hline$C(102)$ & $\mathrm{H}(106)$ & $2.72(5)$ & (1) & $C(102)$ & $\mathrm{H}(104)$ & $3.2(1)$ & (1) \\
\hline$C(102)$ & $\mathrm{H}(102)$ & $2.06(2)$ & (1) & $\mathrm{C}(314)$ & $\mathrm{H}(313)$ & $0.95(2)$ & (1) \\
\hline$C(314)$ & $\mathrm{H}(309)$ & $3.24(5)$ & (1) & C(314) & $\mathrm{H}(311)$ & $3.3(1)$ & (1) \\
\hline$C(314)$ & $\mathrm{H}(312)$ & $2.06(5)$ & (1) & $C(314)$ & $\mathrm{H}(412)$ & $2.9(3)$ & $(2,1,1,0,-2)$ \\
\hline $\mathrm{C}(314)$ & $\mathrm{H}(302)$ & $2.77(6)$ & (1) & $C(314)$ & $\mathrm{H}(404)$ & $3.30(4)$ & (1) \\
\hline$C(604)$ & $\mathrm{H}(614)$ & $2.67(3)$ & (1) & $\mathrm{C}(604)$ & $\mathrm{H}(605)$ & $3.1(1)$ & (1) \\
\hline$C(604)$ & $\mathrm{H}(606)$ & $3.18(7)$ & (1) & C(604) & $\mathrm{H}(608)$ & $2.08(3)$ & (1) \\
\hline C(604) & $\mathrm{H}(607)$ & $2.07(5)$ & (1) & C(604) & $\mathrm{H}(603)$ & $0.95(1)$ & (1) \\
\hline C(604) & $\mathrm{H}(601)$ & $3.5(1)$ & (1) & C(604) & $\mathrm{H}(602)$ & $2.16(4)$ & (1) \\
\hline C(604) & $\mathrm{H}(604)$ & $3.3(1)$ & (1) & C(309) & $\mathrm{H}(313)$ & $2.02(3)$ & (1) \\
\hline C(309) & $\mathrm{H}(309)$ & $2.04(3)$ & (1) & C(309) & $\mathrm{H}(310)$ & $3.3(1)$ & (1) \\
\hline C(309) & $\mathrm{H}(312)$ & $3.3(1)$ & (1) & C(309) & $\mathrm{H}(412)$ & $3.1(2)$ & $(2,1,1,0,-2)$ \\
\hline C(309) & $\mathrm{H}(306)$ & $3.5(1)$ & (1) & C(309) & $\mathrm{H}(301)$ & 2.71(9) & (1) \\
\hline C(309) & $\mathrm{H}(302)$ & $2.8(1)$ & (1) & C(309) & $\mathrm{H}(404)$ & $3.49(9)$ & (1) \\
\hline$C(403)$ & $\mathrm{H}(515)$ & $3.2(1)$ & (1) & C(403) & $\mathrm{H}(413)$ & $2.69(8)$ & (1) \\
\hline$C(403)$ & $\mathrm{H}(308)$ & $3.6(3)$ & $(2,1,1,-1,-2)$ & C(403) & $\mathrm{H}(306)$ & $3.5(3)$ & $(2,1,1,-1,-2)$ \\
\hline$C(403)$ & $\mathrm{H}(408)$ & $2.73(4)$ & (1) & C(403) & $\mathrm{H}(407)$ & $3.33(4)$ & (1) \\
\hline$C(403)$ & $\mathrm{H}(406)$ & $3.2(2)$ & (1) & C(403) & $\mathrm{H}(403)$ & $2.08(6)$ & (1) \\
\hline C(403) & $\mathrm{H}(401)$ & $3.3(1)$ & (1) & C(403) & $\mathrm{H}(402)$ & $0.95(1)$ & (1) \\
\hline$C(403)$ & $\mathrm{H}(110)$ & $3.4(3)$ & $(2,1,0,-1,-2)$ & $C(213)$ & $\mathrm{H}(616)$ & $3.5(3)$ & $(2,1,0,-1,-2)$ \\
\hline$C(213)$ & $\mathrm{H}(504)$ & $3.5(5)$ & $(1,1,-1,0,1) C$ & 13) & $\mathrm{H}(212)$ & $0.95(1)$ & (1) \\
\hline$C(213)$ & $\mathrm{H}(210)$ & $3.3(1)$ & (1) & $C(213)$ & $\mathrm{H}(211)$ & $2.04(3)$ & (1) \\
\hline$C(213)$ & $\mathrm{H}(213)$ & $2.02(3)$ & (1) & $C(213)$ & $\mathrm{H}(102)$ & $3.08(6)$ & (1) \\
\hline$C(316)$ & $\mathrm{H}(607)$ & $3.11(4)$ & (1) & $C(316)$ & $\mathrm{H}(314)$ & $0.95(1)$ & (1) \\
\hline$C(316)$ & $\mathrm{H}(316)$ & $3.27(4)$ & (1) & C(316) & $\mathrm{H}(318)$ & $3.26(5)$ & (1) \\
\hline$C(316)$ & $\mathrm{H}(315)$ & $2.04(6)$ & (1) & $C(316)$ & $\mathrm{H}(303)$ & $2.77(5)$ & (1) \\
\hline$C(316)$ & $\mathrm{H}(208)$ & $3.3(5)$ & $(2,1,0,0,-2)$ & 16) & $\mathrm{H}(203)$ & $3.0(5)$ & $(2,1,0,0,-2)$ \\
\hline $\mathrm{C}(401)$ & H(409) & $2.67(9)$ & (1) & $\mathrm{C}(401)$ & $\mathrm{H}(408)$ & $3.16(5)$ & (1) \\
\hline $\mathrm{C}(401)$ & $\mathrm{H}(407)$ & $3.2(1)$ & (1) & C(401) & $\mathrm{H}(405)$ & $2.06(2)$ & (1) \\
\hline $\mathrm{C}(401)$ & H(406) & $2.07(3)$ & (1) & $\mathrm{C}(401)$ & H(404) & $2.15(3)$ & (1) \\
\hline $\mathrm{C}(401)$ & $\mathrm{H}(403)$ & $3.54(4)$ & (1) & C(401) & $\mathrm{H}(401)$ & $0.95(1)$ & (1) \\
\hline $\mathrm{C}(401)$ & $\mathrm{H}(402)$ & $3.3(1)$ & (1) & $C(405)$ & $\mathrm{H}(316)$ & $3.2(2)$ & $(1,1,1)$ \\
\hline$C(405)$ & $\mathrm{H}(414)$ & $2.66(3)$ & (1) & $C(405)$ & $\mathrm{H}(418)$ & $2.67(3)$ & (1) \\
\hline$C(405)$ & $\mathrm{H}(408)$ & $3.35(5)$ & (1) & $C(405)$ & $\mathrm{H}(407)$ & $2.73(5)$ & (1) \\
\hline$C(405)$ & $\mathrm{H}(405)$ & $3.3(1)$ & (1) & $C(405)$ & $\mathrm{H}(404)$ & $2.06(3)$ & (1) \\
\hline$C(405)$ & $\mathrm{H}(403)$ & $2.08(2)$ & (1) & $C(405)$ & $\mathrm{H}(401)$ & $3.27(3)$ & (1) \\
\hline$C(405)$ & $\mathrm{H}(402)$ & $3.20(5)$ & (1) & C(104) & $\mathrm{H}(114)$ & $2.62(4)$ & (1) \\
\hline$C(104)$ & H(103) & $0.95(1)$ & (1) & C(104) & $\mathrm{H}(101)$ & $3.52(9)$ & (1) \\
\hline C(104) & $\mathrm{H}(108)$ & $2.07(3)$ & (1) & C(104) & H(107) & $2.07(4)$ & (1) \\
\hline C(104) & $\mathrm{H}(105)$ & $3.11(4)$ & (1) & C(104) & $\mathrm{H}(106)$ & $3.14(4)$ & (1) \\
\hline
\end{tabular}




\begin{tabular}{|c|c|c|c|c|c|c|c|}
\hline$C(104)$ & $\mathrm{H}(104)$ & $3.28(6)$ & (1) & $\mathrm{C}(104)$ & $\mathrm{H}(102)$ & $2.17(2)$ & (1) \\
\hline$C(116)$ & $\mathrm{H}(509)$ & $3.1(1)$ & $(1,1,0,0,1)$ & 16) & $\mathrm{H}(204)$ & $3.04(5)$ & (1) \\
\hline$C(116)$ & $\mathrm{H}(201)$ & $2.86(4)$ & (1) & $C(116)$ & $\mathrm{H}(114)$ & $0.95(1)$ & (1) \\
\hline$C(116)$ & $\mathrm{H}(116)$ & $3.3(1)$ & (1) & C(116) & $\mathrm{H}(118)$ & $3.26(5)$ & (1) \\
\hline$C(116)$ & $\mathrm{H}(115)$ & $2.05(4)$ & (1) & C(116) & $\mathrm{H}(103)$ & $2.63(7)$ & (1) \\
\hline$C(116)$ & $\mathrm{H}(107)$ & $3.52(7)$ & (1) & C(109) & $\mathrm{H}(309)$ & $3.4(1)$ & $(1,1,-1)$ \\
\hline C(109) & $\mathrm{H}(310)$ & $3.5(1)$ & $(1,1,-1)$ & C(109) & $\mathrm{H}(113)$ & $2.04(3)$ & (1) \\
\hline C(109) & $\mathrm{H}(110)$ & $3.27(5)$ & (1) & C(109) & $\mathrm{H}(112)$ & $3.27(5)$ & (1) \\
\hline $\mathrm{C}(109)$ & $\mathrm{H}(109)$ & $2.05(3)$ & (1) & C(109) & $\mathrm{H}(101)$ & $2.69(5)$ & (1) \\
\hline C(109) & $\mathrm{H}(106)$ & $3.3(1)$ & (1) & C(109) & $\mathrm{H}(102)$ & $2.75(7)$ & (1) \\
\hline C(410) & $\mathrm{H}(309)$ & $3.58(6)$ & (1) & $C(410)$ & $\mathrm{H}(409)$ & $0.95(1)$ & (1) \\
\hline$C(410)$ & $\mathrm{H}(410)$ & $2.06(5)$ & (1) & $C(410)$ & $\mathrm{H}(411)$ & 3.3(1) & (1) \\
\hline$C(410)$ & $\mathrm{H}(413)$ & $3.22(8)$ & (1) & $C(410)$ & $\mathrm{H}(301)$ & $3.40(5)$ & (1) \\
\hline C(410) & $\mathrm{H}(304)$ & $3.45(8)$ & (1) & $C(410)$ & $\mathrm{H}(401)$ & 2.64(7) & (1) \\
\hline C(413) & $\mathrm{H}(313)$ & $3.5(3)$ & $(2,1,1,-1,-2)$ & C(413) & $\mathrm{H}(412)$ & $0.95(1)$ & (1) \\
\hline C(413) & $\mathrm{H}(410)$ & $3.21(8)$ & (1) & $C(413)$ & $\mathrm{H}(411)$ & $1.99(4)$ & (1) \\
\hline C(413) & $\mathrm{H}(413)$ & $2.03(5)$ & (1) & C(413) & $\mathrm{H}(217)$ & $2.9(1)$ & (1) \\
\hline C(413) & $\mathrm{H}(218)$ & $3.5(1)$ & (1) & C(616) & $\mathrm{H}(514)$ & $3.31(6)$ & (1) \\
\hline$C(616)$ & $\mathrm{H}(614)$ & $0.95(1)$ & (1) & C(616) & $\mathrm{H}(616)$ & $3.27(4)$ & (1) \\
\hline$C(616)$ & $\mathrm{H}(618)$ & $3.2(1)$ & (1) & C(616) & $\mathrm{H}(615)$ & $2.03(3)$ & (1) \\
\hline$C(616)$ & $\mathrm{H}(503)$ & $3.2(1)$ & (1) & C(616) & $\mathrm{H}(603)$ & $2.61(3)$ & (1) \\
\hline$C(616)$ & $\mathrm{H}(502)$ & $3.18(7)$ & (1) & C(616) & $\mathrm{H}(314)$ & $2.9(1)$ & (1) \\
\hline $\mathrm{C}(605)$ & $\mathrm{H}(614)$ & $2.67(7)$ & (1) & $\mathrm{C}(605)$ & $\mathrm{H}(618)$ & $2.66(4)$ & (1) \\
\hline $\mathrm{C}(605)$ & $\mathrm{H}(605)$ & $3.2(1)$ & (1) & $C(605)$ & $\mathrm{H}(608)$ & $3.34(5)$ & (1) \\
\hline $\mathrm{C}(605)$ & $\mathrm{H}(607)$ & $2.75(3)$ & (1) & $\mathrm{C}(605)$ & $\mathrm{H}(603)$ & $2.06(5)$ & (1) \\
\hline$C(605)$ & $\mathrm{H}(601)$ & $3.28(5)$ & (1) & $C(605)$ & $\mathrm{H}(602)$ & $3.17(4)$ & (1) \\
\hline$C(605)$ & $\mathrm{H}(604)$ & $2.07(4)$ & (1) & $C(605)$ & $\mathrm{H}(315)$ & $3.4(1)$ & (1) \\
\hline C(408) & $\mathrm{H}(511)$ & $3.3(3)$ & $(2,1,1,-1,-3)$ & C(408) & $\mathrm{H}(510)$ & $3.6(3)$ & $(2,1,1,-1,-3)$ \\
\hline C(408) & $\mathrm{H}(408)$ & $0.95(1)$ & (1) & C(408) & $\mathrm{H}(407)$ & $0.95(1)$ & (1) \\
\hline C(408) & $\mathrm{H}(405)$ & $2.06(4)$ & (1) & C(408) & $\mathrm{H}(406)$ & $2.06(2)$ & (1) \\
\hline C(408) & $\mathrm{H}(403)$ & $2.08(7)$ & (1) & C(408) & $\mathrm{H}(401)$ & $3.38(8)$ & (1) \\
\hline C(408) & $\mathrm{H}(402)$ & $3.10(5)$ & (1) & C(408) & $\mathrm{H}(215)$ & $3.1(4)$ & $(1,1,1)$ \\
\hline$C(601)$ & $\mathrm{H}(609)$ & $2.73(7)$ & (1) & $C(601)$ & $\mathrm{H}(605)$ & $2.06(3)$ & (1) \\
\hline$C(601)$ & $\mathrm{H}(606)$ & $2.06(3)$ & (1) & $C(601)$ & $\mathrm{H}(608)$ & $3.1(1)$ & (1) \\
\hline$C(601)$ & $\mathrm{H}(607)$ & $3.2(1)$ & (1) & $\mathrm{C}(601)$ & $\mathrm{H}(603)$ & $3.5(1)$ & (1) \\
\hline$C(601)$ & $\mathrm{H}(601)$ & $0.95(1)$ & (1) & $\mathrm{C}(601)$ & $\mathrm{H}(602)$ & $3.29(5)$ & (1) \\
\hline$C(601)$ & $\mathrm{H}(604)$ & $2.17(3)$ & (1) & $C(306)$ & $\mathrm{H}(318)$ & $2.72(3)$ & (1) \\
\hline$C(306)$ & $\mathrm{H}(307)$ & $3.30(5)$ & (1) & C(306) & $\mathrm{H}(306)$ & $3.34(6)$ & (1) \\
\hline$C(306)$ & $\mathrm{H}(305)$ & $2.76(4)$ & (1) & C(306) & $\mathrm{H}(301)$ & $2.07(6)$ & (1) \\
\hline$C(306)$ & $\mathrm{H}(303)$ & $3.26(7)$ & (1) & $C(306)$ & $\mathrm{H}(304)$ & $0.95(1)$ & (1) \\
\hline C(306) & $\mathrm{H}(117)$ & $2.99(8)$ & (1) & $\mathrm{C}(417)$ & $\mathrm{H}(512)$ & $3.4(9)$ & $(2,1,1,-1,-3)$ \\
\hline C(417) & $\mathrm{H}(507)$ & $3.3(1)$ & (1) & $\mathrm{C}(417)$ & $\mathrm{H}(601)$ & $3.2(2)$ & $(1,1,1)$ \\
\hline C(417) & $\mathrm{H}(604)$ & $3.4(1)$ & $(1,1,1)$ & C(417) & $\mathrm{H}(415)$ & $0.95(1)$ & (1) \\
\hline C(417) & $\mathrm{H}(416)$ & $2.04(3)$ & (1) & C(417) & $\mathrm{H}(414)$ & $2.02(4)$ & (1) \\
\hline C(417) & $\mathrm{H}(417)$ & $3.23(5)$ & (1) & $C(417)$ & $\mathrm{H}(210)$ & $3.4(5)$ & $(1,1,1,0,-1)$ \\
\hline $\mathrm{C}(417)$ & $\mathrm{H}(211)$ & $3.0(6)$ & $(1,1,1,0,-1) C$ & 06) & $\mathrm{H}(316)$ & $3.1(3)$ & $(1,1,1)$ \\
\hline C(406) & $\mathrm{H}(418)$ & $2.70(5)$ & (1) & $\mathrm{C}(406)$ & $\mathrm{H}(407)$ & $3.25(5)$ & (1) \\
\hline C(406) & $\mathrm{H}(405)$ & $2.74(8)$ & (1) & C(406) & $\mathrm{H}(406)$ & $3.32(7)$ & (1) \\
\hline C(406) & $\mathrm{H}(404)$ & $0.95(1)$ & (1) & C(406) & $\mathrm{H}(403)$ & $3.25(4)$ & (1) \\
\hline C(406) & $\mathrm{H}(401)$ & $2.05(2)$ & (1) & $C(304)$ & $\mathrm{H}(314)$ & $2.9(1)$ & (1) \\
\hline C(304) & $\mathrm{H}(308)$ & $2.09(3)$ & (1) & C(304) & $\mathrm{H}(307)$ & $2.10(5)$ & (1) \\
\hline$C(304)$ & $\mathrm{H}(306)$ & 3.1(1) & (1) & $C(304)$ & $\mathrm{H}(305)$ & $3.14(5)$ & (1) \\
\hline C(304) & $\mathrm{H}(301)$ & $3.5(2)$ & (1) & $C(304)$ & $\mathrm{H}(303)$ & $0.95(2)$ & (1) \\
\hline
\end{tabular}




\begin{tabular}{|c|c|c|c|c|c|c|c|}
\hline $\mathrm{C}(304)$ & $\mathrm{H}(304)$ & $3.31(4)$ & (1) & $C(304)$ & $\mathrm{H}(302)$ & $2.16(5)$ & (1) \\
\hline $\mathrm{C}(114)$ & $\mathrm{H}(501)$ & $3.5(5)$ & \multicolumn{2}{|c|}{$(1,1,-1,0,1) \mathrm{C}(114)$} & $\mathrm{H}(309)$ & $3.3(1)$ & $(1,1,-1)$ \\
\hline $\mathrm{C}(114)$ & $\mathrm{H}(113)$ & $0.95(1)$ & (1) & $\mathrm{C}(114)$ & $\mathrm{H}(112)$ & $2.04(3)$ & (1) \\
\hline $\mathrm{C}(114)$ & $\mathrm{H}(109)$ & $3.26(5)$ & (1) & $\mathrm{C}(114)$ & $\mathrm{H}(111)$ & $3.28(6)$ & (1) \\
\hline $\mathrm{C}(114)$ & $\mathrm{H}(102)$ & $2.9(1)$ & (1) & $\mathrm{C}(318)$ & $\mathrm{H}(605)$ & $2.88(4)$ & (1) \\
\hline $\mathrm{C}(318)$ & $\mathrm{H}(607)$ & $3.02(9)$ & (1) & $\mathrm{C}(318)$ & $\mathrm{H}(314)$ & $3.26(7)$ & (1) \\
\hline $\mathrm{C}(318)$ & $\mathrm{H}(316)$ & $0.95(1)$ & (1) & $\mathrm{C}(318)$ & $\mathrm{H}(318)$ & $3.3(2)$ & (1) \\
\hline $\mathrm{C}(318)$ & $\mathrm{H}(317)$ & $2.04(3)$ & (1) & $\mathrm{C}(318)$ & $\mathrm{H}(315)$ & $2.02(3)$ & (1) \\
\hline $\mathrm{C}(318)$ & $\mathrm{H}(418)$ & $3.4(4)$ & $(1,1,-1)$ & $\mathrm{C}(318)$ & $\mathrm{H}(405)$ & $3.5(6)$ & $(1,1,-1)$ \\
\hline $\mathrm{C}(318)$ & $\mathrm{H}(404)$ & $3.6(2)$ & $(1,1,-1)$ & $\mathrm{C}(503)$ & $\mathrm{H}(513)$ & $2.69(4)$ & (1) \\
\hline $\mathrm{C}(503)$ & $\mathrm{H}(501)$ & $3.26(9)$ & (1) & $\mathrm{C}(503)$ & $\mathrm{H}(506)$ & $3.3(1)$ & (1) \\
\hline$C(503)$ & $\mathrm{H}(507)$ & $3.34(5)$ & (1) & $\mathrm{C}(503)$ & $\mathrm{H}(508)$ & $2.77(5)$ & (1) \\
\hline$C(503)$ & $\mathrm{H}(503)$ & $2.07(6)$ & (1) & $\mathrm{C}(503)$ & $\mathrm{H}(502)$ & $0.95(1)$ & (1) \\
\hline $\mathrm{C}(610)$ & $\mathrm{H}(512)$ & $3.1(2)$ & $(2,1,0,-1,-3)$ & $C(610)$ & $\mathrm{H}(609)$ & $0.95(1)$ & (1) \\
\hline$C(610)$ & $\mathrm{H}(610)$ & $2.02(4)$ & (1) & $C(610)$ & $\mathrm{H}(613)$ & $3.26(6)$ & (1) \\
\hline$C(610)$ & $\mathrm{H}(611)$ & $3.25(5)$ & (1) & $C(610)$ & $\mathrm{H}(601)$ & $2.67(4)$ & (1) \\
\hline $\mathrm{C}(610)$ & $\mathrm{H}(108)$ & $3.2(5)$ & $(2,1,0,-1,-2)$ & $\mathrm{C}(610)$ & $\mathrm{H}(107)$ & $3.6(4)$ & $(2,1,0,-1,-2)$ \\
\hline$C(610)$ & $\mathrm{H}(106)$ & $3.3(7)$ & $(2,1,0,-1,-2)$ & $C(402)$ & $\mathrm{H}(409)$ & $2.60(4)$ & (1) \\
\hline$C(402)$ & $\mathrm{H}(413)$ & $2.67(3)$ & (1) & $C(402)$ & $\mathrm{H}(308)$ & $3.3(2)$ & $(2,1,1,-1,-2)$ \\
\hline $\mathrm{C}(402)$ & $\mathrm{H}(408)$ & $3.27(7)$ & (1) & $\mathrm{C}(402)$ & $\mathrm{H}(405)$ & $3.33(5)$ & (1) \\
\hline$C(402)$ & $\mathrm{H}(406)$ & $2.71(7)$ & (1) & $\mathrm{C}(402)$ & $\mathrm{H}(404)$ & $3.17(5)$ & (1) \\
\hline $\mathrm{C}(402)$ & $\mathrm{H}(403)$ & $3.3(1)$ & (1) & $\mathrm{C}(402)$ & $\mathrm{H}(401)$ & $2.08(5)$ & (1) \\
\hline $\mathrm{C}(402)$ & $\mathrm{H}(402)$ & $2.07(3)$ & (1) & $\mathrm{C}(515)$ & $\mathrm{H}(515)$ & $3.26(4)$ & (1) \\
\hline $\mathrm{C}(515)$ & $\mathrm{H}(518)$ & $2.04(3)$ & (1) & $\mathrm{C}(515)$ & $\mathrm{H}(514)$ & $2.06(3)$ & (1) \\
\hline $\mathrm{C}(515)$ & $\mathrm{H}(517)$ & $3.28(5)$ & (1) & $\mathrm{C}(515)$ & $\mathrm{H}(507)$ & $3.44(5)$ & (1) \\
\hline $\mathrm{C}(515)$ & $\mathrm{H}(503)$ & $2.69(3)$ & (1) & $\mathrm{C}(515)$ & $\mathrm{H}(602)$ & $3.5(1)$ & (1) \\
\hline $\mathrm{C}(515)$ & $\mathrm{H}(504)$ & 2.79(4) & (1) & $\mathrm{C}(602)$ & $\mathrm{H}(609)$ & $2.67(8)$ & (1) \\
\hline$C(602)$ & $\mathrm{H}(613)$ & $2.67(5)$ & (1) & $\mathrm{C}(602)$ & $\mathrm{H}(605)$ & $3.3(1)$ & (1) \\
\hline $\mathrm{C}(602)$ & $\mathrm{H}(606)$ & $2.76(8)$ & (1) & $C(602)$ & $\mathrm{H}(608)$ & $3.2(1)$ & (1) \\
\hline$C(602)$ & $\mathrm{H}(603)$ & $3.3(1)$ & (1) & $C(602)$ & $\mathrm{H}(601)$ & $2.07(3)$ & (1) \\
\hline$C(602)$ & $\mathrm{H}(602)$ & $2.05(3)$ & (1) & $\mathrm{C}(602)$ & $\mathrm{H}(604)$ & $3.19(4)$ & (1) \\
\hline $\mathrm{C}(602)$ & $\mathrm{H}(106)$ & $3.3(1)$ & $(2,1,0,-1,-2)$ & $\mathrm{C}(411)$ & $\mathrm{H}(409)$ & $2.06(5)$ & (1) \\
\hline $\mathrm{C}(411)$ & $\mathrm{H}(412)$ & $3.22(9)$ & (1) & $\mathrm{C}(411)$ & $\mathrm{H}(410)$ & $0.95(2)$ & (1) \\
\hline $\mathrm{C}(411)$ & $\mathrm{H}(411)$ & $2.04(4)$ & (1) & $\mathrm{C}(411)$ & $\mathrm{H}(301)$ & $3.50(7)$ & (1) \\
\hline $\mathrm{C}(411)$ & $\mathrm{H}(304)$ & $3.3(1)$ & (1) & $\mathrm{C}(411)$ & $\mathrm{H}(116)$ & $2.9(1)$ & (1) \\
\hline $\mathrm{C}(411)$ & $\mathrm{H}(117)$ & $3.36(6)$ & (1) & $\mathrm{C}(517)$ & $\mathrm{H}(515)$ & $0.95(1)$ & (1) \\
\hline $\mathrm{C}(517)$ & $\mathrm{H}(514)$ & $2.01(3)$ & (1) & $\mathrm{C}(517)$ & $\mathrm{H}(517)$ & $3.22(5)$ & (1) \\
\hline $\mathrm{C}(517)$ & $\mathrm{H}(516)$ & $2.03(3)$ & (1) & $\mathrm{C}(517)$ & $\mathrm{H}(603)$ & $3.27(7)$ & (1) \\
\hline $\mathrm{C}(517)$ & $\mathrm{H}(602)$ & $2.99(7)$ & (1) & $\mathrm{C}(517)$ & $\mathrm{H}(414)$ & $3.02(5)$ & (1) \\
\hline $\mathrm{C}(517)$ & $\mathrm{H}(403)$ & $3.5(2)$ & (1) & $\mathrm{C}(517)$ & $\mathrm{H}(110)$ & $3.5(4)$ & $(2,1,0,-1,-2)$ \\
\hline $\mathrm{C}(217)$ & $\mathrm{H}(311)$ & $3.1(3)$ & $(2,1,1,-1,-2)$ & $\mathrm{C}(217)$ & $\mathrm{H}(408)$ & $3.2(5)$ & $(1,1,-1)$ \\
\hline $\mathrm{C}(217)$ & $\mathrm{H}(407)$ & $3.2(7)$ & $(1,1,-1)$ & $\mathrm{C}(217)$ & $\mathrm{H}(405)$ & $3.3(2)$ & $(1,1,-1)$ \\
\hline $\mathrm{C}(217)$ & $\mathrm{H}(406)$ & $3.4(2)$ & $(1,1,-1)$ & $\mathrm{C}(217)$ & $\mathrm{H}(215)$ & $0.95(2)$ & (1) \\
\hline$C(217)$ & $\mathrm{H}(214)$ & $2.03(6)$ & (1) & $\mathrm{C}(217)$ & $\mathrm{H}(216)$ & $2.01(6)$ & (1) \\
\hline$C(217)$ & $\mathrm{H}(217)$ & $3.23(6)$ & (1) & $C(217)$ & $\mathrm{H}(101)$ & $3.4(3)$ & $(2,1,0,-1,-2)$ \\
\hline $\mathrm{C}(217)$ & $\mathrm{H}(104)$ & $3.3(1)$ & $(2,1,0,-1,-2)$ & $\mathrm{C}(619)$ & $\mathrm{H}(610)$ & $2.9(3)$ & $(2,1,0,0,-3)$ \\
\hline $\mathrm{C}(619)$ & $\mathrm{H}(617)$ & $0.95(1)$ & (1) & $\mathrm{C}(619)$ & $\mathrm{H}(616)$ & $2.05(4)$ & (1) \\
\hline $\mathrm{C}(619)$ & $\mathrm{H}(618)$ & $2.03(3)$ & (1) & $\mathrm{C}(619)$ & $\mathrm{H}(615)$ & $3.2(1)$ & (1) \\
\hline $\mathrm{C}(619)$ & $\mathrm{H}(502)$ & $3.4(1)$ & (1) & $C(619)$ & $\mathrm{H}(314)$ & $3.28(4)$ & (1) \\
\hline $\mathrm{C}(619)$ & $\mathrm{H}(206)$ & $3.6(6)$ & $(2,1,0,0,-2)$ & 19) & $\mathrm{H}(202)$ & $3.2(2)$ & $(2,1,0,0,-2)$ \\
\hline $\mathrm{C}(619)$ & $\mathrm{H}(208)$ & $2.9(9)$ & $(2,1,0,0,-2)$ & 18) & $\mathrm{H}(612)$ & $3.5(6)$ & $(2,1,1,0,-3)$ \\
\hline$C(418)$ & $\mathrm{H}(611)$ & $3.2(9)$ & $(2,1,1,0,-3)$ & 18) & $\mathrm{H}(507)$ & $2.9(1)$ & (1) \\
\hline $\mathrm{C}(418)$ & $\mathrm{H}(604)$ & $2.9(1)$ & $(1,1,1)$ & $C(418)$ & $\mathrm{H}(415)$ & $2.03(3)$ & (1) \\
\hline
\end{tabular}




\begin{tabular}{|c|c|c|c|c|c|c|c|}
\hline$C(418)$ & $\mathrm{H}(416)$ & $0.95(1)$ & (1) & $C(418)$ & $\mathrm{H}(414)$ & $3.25(6)$ & (1) \\
\hline C(418) & $\mathrm{H}(417)$ & $2.02(3)$ & (1) & $C(418)$ & $\mathrm{H}(418)$ & $3.26(5)$ & (1) \\
\hline C(418) & $\mathrm{H}(210)$ & $3.4(6)$ & $(1,1,1,0,-1) C$ & 18) & $\mathrm{H}(211)$ & $3.3(7)$ & $(1,1,1,0,-1)$ \\
\hline C(416) & $\mathrm{H}(511)$ & $3.2(2)$ & $(2,1,1,-1,-3)$ & $C(416)$ & $\mathrm{H}(512)$ & $3.4(1)$ & $(2,1,1,-1,-3)$ \\
\hline C(416) & $\mathrm{H}(515)$ & $3.59(7)$ & (1) & $C(416)$ & $\mathrm{H}(601)$ & $3.6(3)$ & $(1,1,1)$ \\
\hline$C(416)$ & $\mathrm{H}(415)$ & $2.01(3)$ & (1) & $C(416)$ & $\mathrm{H}(416)$ & $3.26(8)$ & (1) \\
\hline C(416) & $\mathrm{H}(414)$ & $0.95(1)$ & (1) & $C(416)$ & $\mathrm{H}(418)$ & $3.23(5)$ & (1) \\
\hline C(416) & $\mathrm{H}(403)$ & $2.66(4)$ & (1) & $C(207)$ & $\mathrm{H}(617)$ & $3.4(4)$ & $(2,1,0,-1,-2)$ \\
\hline$C(207)$ & $\mathrm{H}(417)$ & $3.4(3)$ & $(2,1,1,-1,-2)$ & $C(207)$ & $\mathrm{H}(209)$ & $3.42(7)$ & (1) \\
\hline$C(207)$ & $\mathrm{H}(206)$ & $0.95(2)$ & (1) & $C(207)$ & $\mathrm{H}(205)$ & $0.95(1)$ & (1) \\
\hline$C(207)$ & $\mathrm{H}(207)$ & $2.08(3)$ & (1) & $C(207)$ & $\mathrm{H}(208)$ & $2.08(4)$ & (1) \\
\hline$C(207)$ & $\mathrm{H}(203)$ & $3.4(2)$ & (1) & $C(207)$ & $\mathrm{H}(204)$ & $3.12(5)$ & (1) \\
\hline$C(207)$ & $\mathrm{H}(201)$ & $2.09(3)$ & (1) & $C(613)$ & $\mathrm{H}(612)$ & $0.95(1)$ & (1) \\
\hline$C(613)$ & $\mathrm{H}(610)$ & $3.23(6)$ & (1) & $C(613)$ & $\mathrm{H}(613)$ & $2.04(5)$ & (1) \\
\hline$C(613)$ & $\mathrm{H}(611)$ & $2.04(5)$ & (1) & $C(613)$ & $\mathrm{H}(508)$ & $3.5(2)$ & $(2,1,1,-1,-3)$ \\
\hline$C(613)$ & $\mathrm{H}(416)$ & $3.4(4)$ & $(2,1,1,-1,-3)$ & $C(613)$ & $\mathrm{H}(206)$ & $3.5(7)$ & $(1,1,0,0,-1)$ \\
\hline$C(613)$ & $\mathrm{H}(108)$ & $3.1(1)$ & $(2,1,0,-1,-2)$ & $C(618)$ & $\mathrm{H}(614)$ & $3.26(4)$ & (1) \\
\hline C(618) & $\mathrm{H}(617)$ & $2.04(5)$ & (1) & $C(618)$ & $\mathrm{H}(616)$ & $0.95(1)$ & (1) \\
\hline C(618) & $\mathrm{H}(618)$ & $3.27(8)$ & (1) & $C(618)$ & $\mathrm{H}(615)$ & $2.02(4)$ & (1) \\
\hline C(618) & $\mathrm{H}(502)$ & $3.13(9)$ & (1) & $C(618)$ & $\mathrm{H}(314)$ & 3.3(1) & (1) \\
\hline C(618) & $\mathrm{H}(213)$ & $3.4(2)$ & $(2,1,0,0,-2) C$ & 18) & $\mathrm{H}(202)$ & $3.0(2)$ & $(2,1,0,0,-2)$ \\
\hline $\mathrm{C}(310)$ & $\mathrm{H}(313)$ & $3.24(7)$ & (1) & $C(310)$ & $\mathrm{H}(309)$ & $0.95(2)$ & (1) \\
\hline$C(310)$ & $\mathrm{H}(310)$ & $2.06(5)$ & (1) & $C(310)$ & $\mathrm{H}(311)$ & 3.3(1) & (1) \\
\hline$C(310)$ & $\mathrm{H}(409)$ & $3.25(5)$ & (1) & $C(310)$ & $\mathrm{H}(412)$ & $3.6(1)$ & $(2,1,1,0,-2)$ \\
\hline$C(310)$ & $\mathrm{H}(301)$ & $2.64(6)$ & (1) & $C(310)$ & $\mathrm{H}(404)$ & $3.5(1)$ & (1) \\
\hline$C(310)$ & $\mathrm{H}(401)$ & $3.37(5)$ & (1) & $\mathrm{C}(509)$ & $\mathrm{H}(512)$ & $3.27(5)$ & (1) \\
\hline $\mathrm{C}(509)$ & $\mathrm{H}(509)$ & $2.04(3)$ & (1) & $\mathrm{C}(509)$ & $\mathrm{H}(510)$ & 3.3(1) & (1) \\
\hline $\mathrm{C}(509)$ & $\mathrm{H}(513)$ & $2.04(3)$ & (1) & $\mathrm{C}(509)$ & $\mathrm{H}(517)$ & $3.4(2)$ & $(2,1,1,0,-3)$ \\
\hline $\mathrm{C}(509)$ & $\mathrm{H}(516)$ & $3.3(4)$ & $(2,1,1,0,-3) C$ & 99) & $\mathrm{H}(501)$ & $2.72(5)$ & (1) \\
\hline $\mathrm{C}(509)$ & $\mathrm{H}(506)$ & $3.40(6)$ & (1) & $\mathrm{C}(509)$ & $\mathrm{H}(502)$ & $2.76(5)$ & (1) \\
\hline$C(103)$ & $\mathrm{H}(113)$ & $2.88(8)$ & (1) & $C(103)$ & $\mathrm{H}(103)$ & $2.08(6)$ & (1) \\
\hline$C(103)$ & $\mathrm{H}(101)$ & $3.23(4)$ & (1) & $C(103)$ & $\mathrm{H}(108)$ & $2.73(7)$ & (1) \\
\hline$C(103)$ & $\mathrm{H}(107)$ & 3.3(1) & (1) & $C(103)$ & $\mathrm{H}(106)$ & $3.24(4)$ & (1) \\
\hline$C(103)$ & $\mathrm{H}(102)$ & $0.95(1)$ & (1) & $\mathrm{C}(311)$ & $\mathrm{H}(309)$ & $2.05(5)$ & (1) \\
\hline $\mathrm{C}(311)$ & $\mathrm{H}(310)$ & $0.95(2)$ & (1) & $\mathrm{C}(311)$ & $\mathrm{H}(311)$ & $2.04(5)$ & (1) \\
\hline $\mathrm{C}(311)$ & $\mathrm{H}(312)$ & $3.24(6)$ & (1) & $\mathrm{C}(311)$ & $\mathrm{H}(404)$ & $3.18(5)$ & (1) \\
\hline $\mathrm{C}(311)$ & $\mathrm{H}(401)$ & $3.29(5)$ & (1) & $C(305)$ & $\mathrm{H}(314)$ & $2.7(1)$ & (1) \\
\hline$C(305)$ & $\mathrm{H}(318)$ & $2.64(6)$ & (1) & $C(305)$ & $\mathrm{H}(308)$ & $3.36(5)$ & (1) \\
\hline$C(305)$ & $\mathrm{H}(307)$ & $2.75(5)$ & (1) & $C(305)$ & $\mathrm{H}(305)$ & $3.24(6)$ & (1) \\
\hline$C(305)$ & $\mathrm{H}(301)$ & $3.3(2)$ & (1) & $C(305)$ & $\mathrm{H}(303)$ & $2.08(3)$ & (1) \\
\hline$C(305)$ & $\mathrm{H}(304)$ & $2.06(3)$ & (1) & $C(305)$ & $\mathrm{H}(302)$ & $3.20(7)$ & (1) \\
\hline$C(206)$ & $\mathrm{H}(218)$ & $2.69(9)$ & (1) & $C(206)$ & $\mathrm{H}(206)$ & $3.32(5)$ & (1) \\
\hline$C(206)$ & $\mathrm{H}(205)$ & $2.77(7)$ & (1) & $C(206)$ & $\mathrm{H}(207)$ & $3.24(4)$ & (1) \\
\hline$C(206)$ & $\mathrm{H}(203)$ & $3.23(6)$ & (1) & $C(206)$ & $\mathrm{H}(204)$ & $0.95(1)$ & (1) \\
\hline$C(206)$ & $\mathrm{H}(201)$ & $2.06(3)$ & (1) & $C(101)$ & $\mathrm{H}(606)$ & $3.6(5)$ & $(2,1,0,0,-2)$ \\
\hline $\mathrm{C}(101)$ & $\mathrm{H}(310)$ & $3.6(1)$ & $(1,1,-1)$ & $C(101)$ & $\mathrm{H}(215)$ & $3.5(2)$ & $(2,1,0,0,-2)$ \\
\hline $\mathrm{C}(101)$ & $\mathrm{H}(216)$ & $3.4(5)$ & $(2,1,0,0,-2) C$ & 01) & H(109) & $2.58(4)$ & (1) \\
\hline $\mathrm{C}(101)$ & $\mathrm{H}(103)$ & $3.52(9)$ & (1) & $C(101)$ & $\mathrm{H}(101)$ & $0.95(1)$ & (1) \\
\hline $\mathrm{C}(101)$ & $\mathrm{H}(108)$ & $3.11(7)$ & (1) & $C(101)$ & $\mathrm{H}(107)$ & $3.1(1)$ & (1) \\
\hline $\mathrm{C}(101)$ & $\mathrm{H}(105)$ & $2.03(5)$ & (1) & $C(101)$ & H(106) & $2.04(3)$ & (1) \\
\hline $\mathrm{C}(101)$ & $\mathrm{H}(104)$ & $2.16(6)$ & (1) & $\mathrm{C}(101)$ & $\mathrm{H}(102)$ & $3.28(7)$ & (1) \\
\hline$C(216)$ & $\mathrm{H}(311)$ & $2.9(5)$ & $(2,1,1,-1,-2)$ & $C(216)$ & $\mathrm{H}(307)$ & $3.4(1)$ & $(2,1,0,-1,-2)$ \\
\hline$C(216)$ & $\mathrm{H}(405)$ & $3.4(5)$ & $(1,1,-1)$ & $C(216)$ & H(406) & $3.1(3)$ & $(1,1,-1)$ \\
\hline
\end{tabular}




\begin{tabular}{|c|c|c|c|c|c|c|c|}
\hline $\mathrm{C}(216)$ & $\mathrm{H}(215)$ & $2.04(3)$ & (1) & $C(216)$ & $\mathrm{H}(214)$ & $0.95(2)$ & (1) \\
\hline $\mathrm{C}(216)$ & $\mathrm{H}(216)$ & $3.2(1)$ & (1) & $C(216)$ & $\mathrm{H}(218)$ & $3.25(9)$ & (1) \\
\hline$C(216)$ & $\mathrm{H}(203)$ & $2.74(7)$ & (1) & $\mathrm{C}(111)$ & $\mathrm{H}(516)$ & $3.2(4)$ & $(2,1,0,0,-2)$ \\
\hline $\mathrm{C}(111)$ & $\mathrm{H}(413)$ & $3.4(2)$ & \multicolumn{2}{|c|}{$(2,1,0,0,-2) C(111)$} & $\mathrm{H}(306)$ & $3.4(9)$ & $(1,1,-1)$ \\
\hline $\mathrm{C}(111)$ & $\mathrm{H}(402)$ & $3.3(3)$ & \multicolumn{2}{|c|}{$(2,1,0,0,-2) \mathrm{C}(111)$} & $\mathrm{H}(110)$ & $0.95(2)$ & (1) \\
\hline $\mathrm{C}(111)$ & $\mathrm{H}(112)$ & $3.21(6)$ & (1) & $\mathrm{C}(111)$ & $\mathrm{H}(109)$ & $2.04(3)$ & (1) \\
\hline $\mathrm{C}(111)$ & $\mathrm{H}(111)$ & $2.01(3)$ & (1) & $\mathrm{C}(218)$ & $\mathrm{H}(317)$ & $3.32(6)$ & (1) \\
\hline $\mathrm{C}(218)$ & $\mathrm{H}(407)$ & $3.5(5)$ & $(1,1,-1)$ & $\mathrm{C}(218)$ & $\mathrm{H}(215)$ & $2.00(4)$ & (1) \\
\hline $\mathrm{C}(218)$ & $\mathrm{H}(214)$ & $3.2(1)$ & (1) & $\mathrm{C}(218)$ & $\mathrm{H}(216)$ & $0.95(2)$ & (1) \\
\hline $\mathrm{C}(218)$ & $\mathrm{H}(217)$ & $2.02(5)$ & (1) & $\mathrm{C}(218)$ & $\mathrm{H}(218)$ & $3.2(1)$ & (1) \\
\hline $\mathrm{C}(218)$ & $\mathrm{H}(101)$ & $3.1(4)$ & $(2,1,0,-1,-2)$ & $C(419)$ & $\mathrm{H}(611)$ & $3.5(1)$ & $(2,1,1,0,-3)$ \\
\hline C(419) & $\mathrm{H}(507)$ & $3.5(2)$ & (1) & $\mathrm{C}(419)$ & $\mathrm{H}(604)$ & $3.3(4)$ & $(1,1,1)$ \\
\hline C(419) & $\mathrm{H}(315)$ & $3.3(5)$ & $(1,1,1)$ & C(419) & $\mathrm{H}(415)$ & $3.22(5)$ & (1) \\
\hline C(419) & $\mathrm{H}(416)$ & $2.03(3)$ & (1) & C(419) & $\mathrm{H}(417)$ & $0.95(1)$ & (1) \\
\hline$C(419)$ & $\mathrm{H}(418)$ & $2.04(3)$ & (1) & $C(419)$ & $\mathrm{H}(207)$ & $3.5(3)$ & $(2,1,1,0,-2)$ \\
\hline C(308) & $\mathrm{H}(308)$ & $0.95(1)$ & (1) & C(308) & $\mathrm{H}(307)$ & $0.95(1)$ & (1) \\
\hline $\mathrm{C}(308)$ & $\mathrm{H}(306)$ & $2.06(7)$ & (1) & C(308) & $\mathrm{H}(305)$ & $2.06(4)$ & (1) \\
\hline $\mathrm{C}(308)$ & $\mathrm{H}(301)$ & $3.38(8)$ & (1) & C(308) & $\mathrm{H}(303)$ & $2.12(4)$ & (1) \\
\hline $\mathrm{C}(308)$ & $\mathrm{H}(302)$ & $3.1(1)$ & (1) & $\mathrm{C}(308)$ & $\mathrm{H}(408)$ & $3.4(4)$ & $(2,1,1,0,-2)$ \\
\hline $\mathrm{C}(308)$ & $\mathrm{H}(406)$ & $3.2(1)$ & \multicolumn{2}{|c|}{$(2,1,1,0,-2) \mathrm{C}(308)$} & $\mathrm{H}(214)$ & $3.4(2)$ & $(2,1,0,0,-2)$ \\
\hline $\mathrm{C}(312)$ & $\mathrm{H}(313)$ & $3.3(1)$ & (1) & $\mathrm{C}(312)$ & $\mathrm{H}(309)$ & $3.3(1)$ & (1) \\
\hline $\mathrm{C}(312)$ & $\mathrm{H}(310)$ & $2.04(3)$ & (1) & $\mathrm{C}(312)$ & $\mathrm{H}(311)$ & $0.95(1)$ & (1) \\
\hline$C(312)$ & $\mathrm{H}(312)$ & $2.00(3)$ & (1) & $C(312)$ & $\mathrm{H}(404)$ & $2.98(5)$ & (1) \\
\hline $\mathrm{C}(118)$ & $\mathrm{H}(509)$ & $3.1(3)$ & $(1,1,0,0,1) C$ & 18) & $\mathrm{H}(410)$ & 3.1(1) & (1) \\
\hline C(118) & $\mathrm{H}(411)$ & $3.13(6)$ & (1) & $\mathrm{C}(118)$ & $\mathrm{H}(204)$ & $3.12(5)$ & (1) \\
\hline C(118) & $\mathrm{H}(114)$ & $3.3(1)$ & (1) & $C(118)$ & $\mathrm{H}(116)$ & $0.95(1)$ & (1) \\
\hline $\mathrm{C}(118)$ & H(118) & $3.3(1)$ & (1) & $C(118)$ & $\mathrm{H}(117)$ & $2.05(3)$ & (1) \\
\hline $\mathrm{C}(118)$ & $\mathrm{H}(115)$ & $2.05(3)$ & (1) & $C(118)$ & $\mathrm{H}(112)$ & $3.1(2)$ & $(1,1,1)$ \\
\hline C(603) & $\mathrm{H}(613)$ & $2.67(4)$ & (1) & $C(603)$ & $\mathrm{H}(606)$ & $3.3(1)$ & (1) \\
\hline $\mathrm{C}(603)$ & $\mathrm{H}(608)$ & $2.73(4)$ & (1) & $C(603)$ & $\mathrm{H}(607)$ & $3.33(8)$ & (1) \\
\hline $\mathrm{C}(603)$ & $\mathrm{H}(603)$ & $2.06(4)$ & (1) & $C(603)$ & $\mathrm{H}(601)$ & $3.24(4)$ & (1) \\
\hline $\mathrm{C}(603)$ & $\mathrm{H}(602)$ & $0.95(1)$ & (1) & $\mathrm{C}(507)$ & $\mathrm{H}(517)$ & $3.6(1)$ & $(2,1,1,0,-3)$ \\
\hline $\mathrm{C}(507)$ & $\mathrm{H}(612)$ & $3.3(2)$ & \multicolumn{2}{|c|}{$(2,1,1,0,-3) \mathrm{C}(507)$} & $\mathrm{H}(501)$ & $2.10(7)$ & (1) \\
\hline $\mathrm{C}(507)$ & $\mathrm{H}(506)$ & $0.95(1)$ & (1) & $\mathrm{C}(507)$ & $\mathrm{H}(505)$ & $0.95(1)$ & (1) \\
\hline $\mathrm{C}(507)$ & $\mathrm{H}(507)$ & $2.06(5)$ & (1) & $C(507)$ & $\mathrm{H}(508)$ & $2.07(3)$ & (1) \\
\hline$C(507)$ & $\mathrm{H}(503)$ & $3.4(1)$ & (1) & $\mathrm{C}(507)$ & $\mathrm{H}(504)$ & $3.11(4)$ & (1) \\
\hline $\mathrm{C}(507)$ & $\mathrm{H}(211)$ & $3.5(6)$ & \multicolumn{2}{|c|}{$(1,1,1,0,-1) \mathrm{C}(415)$} & $\mathrm{H}(316)$ & $3.1(6)$ & $(1,1,1)$ \\
\hline C(415) & $\mathrm{H}(415)$ & $3.25(5)$ & (1) & $\mathrm{C}(415)$ & $\mathrm{H}(414)$ & $2.03(3)$ & (1) \\
\hline C(415) & $\mathrm{H}(417)$ & $3.27(6)$ & (1) & $\mathrm{C}(415)$ & $\mathrm{H}(418)$ & $2.03(3)$ & (1) \\
\hline$C(415)$ & $\mathrm{H}(407)$ & $3.45(9)$ & (1) & $\mathrm{C}(415)$ & $\mathrm{H}(404)$ & $2.78(3)$ & (1) \\
\hline $\mathrm{C}(415)$ & $\mathrm{H}(403)$ & $2.72(3)$ & (1) & $\mathrm{C}(620)$ & $\mathrm{H}(610)$ & $3.2(3)$ & $(2,1,0,0,-3)$ \\
\hline $\mathrm{C}(620)$ & $\mathrm{H}(611)$ & $3.2(2)$ & \multicolumn{2}{|c|}{$(2,1,0,0,-3) \mathrm{C}(620)$} & $\mathrm{H}(614)$ & $3.2(1)$ & (1) \\
\hline $\mathrm{C}(620)$ & $\mathrm{H}(617)$ & $2.04(2)$ & (1) & $\mathrm{C}(620)$ & $\mathrm{H}(616)$ & $3.28(6)$ & (1) \\
\hline $\mathrm{C}(620)$ & $\mathrm{H}(618)$ & $0.95(1)$ & (1) & $\mathrm{C}(620)$ & $\mathrm{H}(604)$ & $2.78(4)$ & (1) \\
\hline$C(620)$ & $\mathrm{H}(502)$ & $3.6(2)$ & (1) & $\mathrm{C}(620)$ & $\mathrm{H}(314)$ & $3.13(6)$ & (1) \\
\hline $\mathrm{C}(620)$ & $\mathrm{H}(315)$ & $3.1(1)$ & (1) & $\mathrm{C}(620)$ & $\mathrm{H}(208)$ & $3.0(7)$ & $(2,1,0,0,-2)$ \\
\hline$C(606)$ & $\mathrm{H}(618)$ & $2.70(3)$ & (1) & C(606) & $\mathrm{H}(605)$ & $2.69(6)$ & (1) \\
\hline$C(606)$ & $\mathrm{H}(606)$ & $3.32(4)$ & (1) & C(606) & $\mathrm{H}(607)$ & 3.3(1) & (1) \\
\hline C(606) & $\mathrm{H}(603)$ & $3.2(1)$ & (1) & $\mathrm{C}(606)$ & $\mathrm{H}(601)$ & $2.08(2)$ & (1) \\
\hline$C(606)$ & $\mathrm{H}(604)$ & $0.95(1)$ & (1) & C(606) & $\mathrm{H}(315)$ & $3.40(6)$ & (1) \\
\hline$C(606)$ & $\mathrm{H}(210)$ & $3.6(1)$ & $(1,1,0,0,-1) C$ & 05) & $\mathrm{H}(518)$ & $2.68(4)$ & (1) \\
\hline $\mathrm{C}(505)$ & $\mathrm{H}(514)$ & $2.71(4)$ & (1) & $C(505)$ & $\mathrm{H}(501)$ & $3.28(4)$ & (1) \\
\hline $\mathrm{C}(505)$ & $\mathrm{H}(505)$ & $3.3(1)$ & (1) & $C(505)$ & $\mathrm{H}(507)$ & $2.69(4)$ & (1) \\
\hline
\end{tabular}




\begin{tabular}{|c|c|c|c|c|c|c|c|}
\hline$C(505)$ & $\mathrm{H}(508)$ & $3.31(5)$ & (1) & $C(505)$ & $\mathrm{H}(503)$ & $2.06(3)$ & (1) \\
\hline$C(505)$ & $\mathrm{H}(502)$ & $3.15(4)$ & (1) & $C(505)$ & $\mathrm{H}(504)$ & $2.08(3)$ & (1) \\
\hline$C(210)$ & $\mathrm{H}(209)$ & $0.95(1)$ & (1) & $C(210)$ & $\mathrm{H}(210)$ & $2.02(3)$ & (1) \\
\hline$C(210)$ & $\mathrm{H}(211)$ & $3.3(1)$ & (1) & $C(210)$ & $\mathrm{H}(213)$ & $3.3(1)$ & (1) \\
\hline$C(210)$ & $H(206)$ & $3.50(7)$ & (1) & $C(210)$ & $\mathrm{H}(201)$ & $2.65(9)$ & (1) \\
\hline$C(210)$ & $\mathrm{H}(114)$ & $3.6(1)$ & (1) & $\mathrm{C}(210)$ & $\mathrm{H}(103)$ & $2.89(5)$ & (1) \\
\hline$C(210)$ & $\mathrm{H}(102)$ & $3.24(7)$ & (1) & $\mathrm{C}(108)$ & $\mathrm{H}(114)$ & $3.45(5)$ & (1) \\
\hline $\mathrm{C}(108)$ & $\mathrm{H}(103)$ & $2.10(3)$ & (1) & C(108) & $\mathrm{H}(101)$ & $3.3(1)$ & (1) \\
\hline $\mathrm{C}(108)$ & $\mathrm{H}(108)$ & $0.95(2)$ & (1) & C(108) & $\mathrm{H}(107)$ & $0.95(1)$ & (1) \\
\hline $\mathrm{C}(108)$ & $\mathrm{H}(105)$ & $2.05(3)$ & (1) & C(108) & $\mathrm{H}(106)$ & $2.05(3)$ & (1) \\
\hline $\mathrm{C}(108)$ & $\mathrm{H}(102)$ & $3.11(6)$ & (1) & $C(412)$ & $\mathrm{H}(409)$ & $3.28(7)$ & (1) \\
\hline$C(412)$ & $\mathrm{H}(412)$ & $1.99(4)$ & (1) & C(412) & $\mathrm{H}(410)$ & $2.03(5)$ & (1) \\
\hline$C(412)$ & $\mathrm{H}(411)$ & $0.95(2)$ & (1) & C(412) & $\mathrm{H}(413)$ & $3.21(8)$ & (1) \\
\hline$C(412)$ & $\mathrm{H}(304)$ & $3.56(7)$ & (1) & C(412) & $\mathrm{H}(217)$ & $3.4(2)$ & (1) \\
\hline$C(412)$ & $\mathrm{H}(218)$ & $3.27(5)$ & (1) & $C(412)$ & $\mathrm{H}(116)$ & $3.09(6)$ & (1) \\
\hline$C(412)$ & $\mathrm{H}(117)$ & $3.47(6)$ & (1) & C(611) & $\mathrm{H}(512)$ & $3.5(2)$ & $(2,1,0,-1,-3)$ \\
\hline$C(611)$ & $\mathrm{H}(609)$ & $2.03(3)$ & (1) & C(611) & $\mathrm{H}(612)$ & $3.23(5)$ & (1) \\
\hline$C(611)$ & $\mathrm{H}(610)$ & $0.95(1)$ & (1) & C(611) & $\mathrm{H}(611)$ & $2.02(3)$ & (1) \\
\hline$C(611)$ & $\mathrm{H}(617)$ & $3.0(2)$ & $(2,1,0,-1,-3)$ & $C(611)$ & $\mathrm{H}(618)$ & $3.4(1)$ & $(2,1,0,-1,-3)$ \\
\hline$C(611)$ & $H(206)$ & $3.2(5)$ & $(1,1,0,0,-1) \mathrm{C}$ & 11) & $\mathrm{H}(108)$ & $2.9(4)$ & $(2,1,0,-1,-2)$ \\
\hline$C(611)$ & $\mathrm{H}(107)$ & $3.4(5)$ & $(2,1,0,-1,-2)$ & C(106) & $\mathrm{H}(310)$ & $3.4(8)$ & $(1,1,-1)$ \\
\hline$C(106)$ & $\mathrm{H}(215)$ & $3.2(1)$ & $(2,1,0,0,-2) \mathrm{C}$ & 06) & $\mathrm{H}(118)$ & $2.72(8)$ & (1) \\
\hline$C(106)$ & $\mathrm{H}(103)$ & $3.23(4)$ & (1) & C(106) & $\mathrm{H}(101)$ & $2.06(3)$ & (1) \\
\hline$C(106)$ & $\mathrm{H}(107)$ & $3.2(1)$ & (1) & C(106) & $\mathrm{H}(105)$ & $2.69(4)$ & (1) \\
\hline$C(106)$ & $\mathrm{H}(106)$ & $3.30(9)$ & (1) & C(106) & $\mathrm{H}(104)$ & $0.95(2)$ & (1) \\
\hline$C(520)$ & $\mathrm{H}(518)$ & $0.95(1)$ & (1) & $C(520)$ & $\mathrm{H}(514)$ & $3.26(5)$ & (1) \\
\hline$C(520)$ & $\mathrm{H}(517)$ & $2.02(3)$ & (1) & $C(520)$ & $\mathrm{H}(516)$ & $3.28(5)$ & (1) \\
\hline$C(520)$ & $\mathrm{H}(504)$ & $2.78(4)$ & (1) & C(113) & $\mathrm{H}(509)$ & $3.3(5)$ & $(1,1,-1,0,1)$ \\
\hline$C(113)$ & $\mathrm{H}(501)$ & $2.9(6)$ & $(1,1,-1,0,1) \mathrm{C}$ & 13) & $\mathrm{H}(309)$ & $3.4(1)$ & $(1,1,-1)$ \\
\hline$C(113)$ & $\mathrm{H}(301)$ & $3.3(1)$ & $(1,1,-1)$ & $\mathrm{C}(113)$ & $\mathrm{H}(113)$ & $2.05(3)$ & (1) \\
\hline$C(113)$ & $\mathrm{H}(110)$ & $3.21(6)$ & (1) & $\mathrm{C}(113)$ & $\mathrm{H}(112)$ & $0.95(2)$ & (1) \\
\hline$C(113)$ & $\mathrm{H}(111)$ & $2.04(4)$ & (1) & $\mathrm{C}(211)$ & $\mathrm{H}(609)$ & $3.4(6)$ & $(1,1,0,0,1)$ \\
\hline$C(211)$ & $\mathrm{H}(415)$ & $3.1(6)$ & $(1,1,-1,0,1) \mathrm{C}$ & 11) & $\mathrm{H}(416)$ & $3.5(6)$ & $(1,1,-1,0,1)$ \\
\hline$C(211)$ & $\mathrm{H}(212)$ & $3.3(1)$ & (1) & $\mathrm{C}(211)$ & $\mathrm{H}(209)$ & $2.03(5)$ & (1) \\
\hline$C(211)$ & $\mathrm{H}(210)$ & $0.95(2)$ & (1) & $C(211)$ & $\mathrm{H}(211)$ & $2.05(4)$ & (1) \\
\hline$C(211)$ & $\mathrm{H}(103)$ & $3.2(1)$ & (1) & $\mathrm{C}(211)$ & $\mathrm{H}(102)$ & $3.15(6)$ & (1) \\
\hline$C(105)$ & $\mathrm{H}(114)$ & $2.64(8)$ & (1) & C(105) & $\mathrm{H}(118)$ & $2.7(1)$ & (1) \\
\hline$C(105)$ & $\mathrm{H}(103)$ & $2.06(3)$ & (1) & $C(105)$ & $\mathrm{H}(101)$ & $3.26(6)$ & (1) \\
\hline$C(105)$ & $\mathrm{H}(108)$ & $3.32(8)$ & (1) & C(105) & $\mathrm{H}(107)$ & $2.71(4)$ & (1) \\
\hline$C(105)$ & $\mathrm{H}(105)$ & $3.18(4)$ & (1) & C(105) & $\mathrm{H}(104)$ & $2.05(3)$ & (1) \\
\hline$C(105)$ & $\mathrm{H}(102)$ & $3.2(1)$ & (1) & $C(511)$ & $\mathrm{H}(511)$ & $2.04(3)$ & (1) \\
\hline$C(511)$ & $\mathrm{H}(512)$ & $3.26(7)$ & (1) & $\mathrm{C}(511)$ & $\mathrm{H}(509)$ & $2.06(3)$ & (1) \\
\hline$C(511)$ & $\mathrm{H}(510)$ & $0.95(2)$ & (1) & $\mathrm{C}(511)$ & $\mathrm{H}(403)$ & $3.2(2)$ & $(2,1,1,0,-3)$ \\
\hline$C(511)$ & $\mathrm{H}(111)$ & $3.2(5)$ & $(1,1,1,0,-1) \mathrm{C}$ & & $\mathrm{H}(107)$ & $3.0(2)$ & $(1,1,0,0,-1)$ \\
\hline$C(511)$ & $\mathrm{H}(105)$ & $3.3(2)$ & $(1,1,0,0,-1) \mathrm{C}$ & 14) & $\mathrm{H}(409)$ & $3.23(8)$ & (1) \\
\hline$C(414)$ & $\mathrm{H}(412)$ & $2.03(5)$ & (1) & $\mathrm{C}(414)$ & $\mathrm{H}(411)$ & $3.2(1)$ & (1) \\
\hline$C(414)$ & $\mathrm{H}(413)$ & $0.95(1)$ & (1) & $C(414)$ & $\mathrm{H}(308)$ & $3.1(1)$ & $(2,1,1,-1,-2)$ \\
\hline$C(414)$ & $\mathrm{H}(306)$ & $3.1(1)$ & $(2,1,1,-1,-2)$ & C(414) & $\mathrm{H}(402)$ & $2.75(9)$ & (1) \\
\hline$C(203)$ & $\mathrm{H}(617)$ & $3.2(1)$ & $(2,1,0,-1,-2)$ & $C(203)$ & $\mathrm{H}(616)$ & $3.5(2)$ & $(2,1,0,-1,-2)$ \\
\hline$C(203)$ & $H(213)$ & $2.71(8)$ & (1) & C(203) & $\mathrm{H}(206)$ & $3.2(2)$ & (1) \\
\hline$C(203)$ & $\mathrm{H}(202)$ & $0.95(1)$ & (1) & $C(203)$ & $\mathrm{H}(207)$ & $3.32(5)$ & (1) \\
\hline$C(203)$ & $\mathrm{H}(208)$ & $2.72(5)$ & (1) & $C(203)$ & $\mathrm{H}(203)$ & $2.07(4)$ & (1) \\
\hline$C(203)$ & $\mathrm{H}(201)$ & $3.2(1)$ & (1) & $C(315)$ & $\mathrm{H}(607)$ & $3.33(5)$ & (1) \\
\hline
\end{tabular}




\begin{tabular}{|c|c|c|c|c|c|c|c|}
\hline$C(315)$ & $\mathrm{H}(314)$ & $2.04(3)$ & (1) & $C(315)$ & $\mathrm{H}(318)$ & $2.04(3)$ & (1) \\
\hline$C(315)$ & $\mathrm{H}(317)$ & $3.25(7)$ & (1) & $\mathrm{C}(315)$ & $\mathrm{H}(315)$ & $3.3(2)$ & (1) \\
\hline$C(315)$ & $\mathrm{H}(307)$ & $3.39(6)$ & (1) & $C(315)$ & $\mathrm{H}(303)$ & $2.71(3)$ & (1) \\
\hline$C(315)$ & $\mathrm{H}(304)$ & $2.8(1)$ & (1) & $\mathrm{C}(208)$ & $\mathrm{H}(617)$ & $3.4(5)$ & $(2,1,0,-1,-2)$ \\
\hline $\mathrm{C}(208)$ & $\mathrm{H}(312)$ & $3.3(2)$ & $(2,1,1,-1,-2)$ & $C(208)$ & $\mathrm{H}(315)$ & $3.0(2)$ & $(2,1,0,-1,-2)$ \\
\hline$C(208)$ & $\mathrm{H}(417)$ & $3.1(3)$ & $(2,1,1,-1,-2)$ & $\mathrm{C}(208)$ & $\mathrm{H}(206)$ & $2.08(4)$ & (1) \\
\hline $\mathrm{C}(208)$ & $\mathrm{H}(205)$ & $2.09(6)$ & (1) & $C(208)$ & $\mathrm{H}(202)$ & $3.09(5)$ & (1) \\
\hline$C(208)$ & $\mathrm{H}(207)$ & $0.95(2)$ & (1) & $\mathrm{C}(208)$ & $\mathrm{H}(208)$ & $0.95(2)$ & (1) \\
\hline $\mathrm{C}(208)$ & $\mathrm{H}(203)$ & $2.06(7)$ & (1) & $C(208)$ & $\mathrm{H}(201)$ & $3.37(5)$ & (1) \\
\hline $\mathrm{C}(301)$ & $\mathrm{H}(309)$ & $2.67(6)$ & (1) & C(301) & $\mathrm{H}(308)$ & $3.17(5)$ & (1) \\
\hline $\mathrm{C}(301)$ & $\mathrm{H}(307)$ & $3.16(4)$ & (1) & $\mathrm{C}(301)$ & $\mathrm{H}(306)$ & $2.05(3)$ & (1) \\
\hline $\mathrm{C}(301)$ & $\mathrm{H}(305)$ & $2.05(5)$ & (1) & C(301) & $\mathrm{H}(301)$ & $0.95(2)$ & (1) \\
\hline $\mathrm{C}(301)$ & $\mathrm{H}(303)$ & $3.5(2)$ & (1) & C(301) & $\mathrm{H}(304)$ & $2.17(3)$ & (1) \\
\hline $\mathrm{C}(301)$ & $\mathrm{H}(302)$ & $3.29(4)$ & (1) & C(301) & $\mathrm{H}(117)$ & $3.3(1)$ & (1) \\
\hline$C(215)$ & $\mathrm{H}(311)$ & $3.3(7)$ & $(2,1,1,-1,-2)$ & $C(215)$ & $\mathrm{H}(312)$ & $3.3(1)$ & $(2,1,1,-1,-2)$ \\
\hline$C(215)$ & $H(215)$ & $3.3(1)$ & (1) & $C(215)$ & $\mathrm{H}(214)$ & $2.06(4)$ & (1) \\
\hline$C(215)$ & $\mathrm{H}(217)$ & $3.2(1)$ & (1) & $C(215)$ & $\mathrm{H}(218)$ & $2.01(3)$ & (1) \\
\hline$C(215)$ & $\mathrm{H}(207)$ & $3.55(6)$ & (1) & $C(215)$ & $\mathrm{H}(203)$ & $2.76(8)$ & (1) \\
\hline$C(215)$ & $\mathrm{H}(204)$ & $2.77(7)$ & (1) & $\mathrm{C}(209)$ & $\mathrm{H}(617)$ & $3.3(1)$ & $(2,1,0,-1,-2)$ \\
\hline$C(209)$ & $\mathrm{H}(212)$ & $3.29(8)$ & (1) & C(209) & $\mathrm{H}(209)$ & $2.04(6)$ & (1) \\
\hline$C(209)$ & $H(210)$ & $3.27(6)$ & (1) & C(209) & $\mathrm{H}(213)$ & $2.05(5)$ & (1) \\
\hline$C(209)$ & $\mathrm{H}(206)$ & $3.33(5)$ & (1) & C(209) & $\mathrm{H}(202)$ & $2.77(5)$ & (1) \\
\hline$C(209)$ & $\mathrm{H}(201)$ & $2.69(4)$ & (1) & C(209) & $\mathrm{H}(103)$ & $3.51(4)$ & (1) \\
\hline$C(209)$ & $\mathrm{H}(102)$ & $3.24(4)$ & (1) & $C(404)$ & $\mathrm{H}(511)$ & $3.2(2)$ & $(2,1,1,-1,-3)$ \\
\hline C(404) & $\mathrm{H}(515)$ & $3.5(2)$ & (1) & C(404) & $\mathrm{H}(414)$ & $2.71(3)$ & (1) \\
\hline C(404) & $\mathrm{H}(408)$ & $2.06(4)$ & (1) & $C(404)$ & $\mathrm{H}(407)$ & $2.05(3)$ & (1) \\
\hline C(404) & $\mathrm{H}(405)$ & $3.1(1)$ & (1) & C(404) & H(406) & $3.1(1)$ & (1) \\
\hline C(404) & $\mathrm{H}(404)$ & $3.29(4)$ & (1) & C(404) & H(403) & $0.95(1)$ & (1) \\
\hline$C(404)$ & $\mathrm{H}(401)$ & $3.54(4)$ & (1) & C(404) & $\mathrm{H}(402)$ & $2.18(4)$ & (1) \\
\hline C(407) & $\mathrm{H}(317)$ & $3.4(2)$ & $(1,1,1)$ & $C(407)$ & $\mathrm{H}(308)$ & $3.4(2)$ & $(2,1,1,-1,-2)$ \\
\hline C(407) & $\mathrm{H}(408)$ & $2.06(3)$ & (1) & C(407) & H(407) & $2.07(4)$ & (1) \\
\hline C(407) & $\mathrm{H}(405)$ & $0.95(1)$ & (1) & C(407) & H(406) & $0.95(1)$ & (1) \\
\hline $\mathrm{C}(407)$ & $\mathrm{H}(404)$ & $3.12(4)$ & (1) & C(407) & $\mathrm{H}(403)$ & $3.3(1)$ & (1) \\
\hline $\mathrm{C}(407)$ & $\mathrm{H}(401)$ & $2.10(4)$ & (1) & $C(407)$ & $\mathrm{H}(215)$ & $3.6(2)$ & $(1,1,1)$ \\
\hline C(407) & $\mathrm{H}(214)$ & $3.6(6)$ & $(1,1,1)$ & $C(302)$ & $\mathrm{H}(313)$ & $2.65(8)$ & (1) \\
\hline$C(302)$ & $\mathrm{H}(309)$ & $2.7(1)$ & (1) & $C(302)$ & $\mathrm{H}(308)$ & $3.29(6)$ & (1) \\
\hline$C(302)$ & $\mathrm{H}(306)$ & $2.70(6)$ & (1) & $C(302)$ & $\mathrm{H}(305)$ & $3.3(1)$ & (1) \\
\hline$C(302)$ & $\mathrm{H}(301)$ & $2.07(3)$ & (1) & $C(302)$ & $\mathrm{H}(303)$ & $3.3(1)$ & (1) \\
\hline$C(302)$ & $\mathrm{H}(304)$ & $3.17(6)$ & (1) & $C(302)$ & $\mathrm{H}(302)$ & $2.06(3)$ & (1) \\
\hline$C(313)$ & $\mathrm{H}(313)$ & $2.06(5)$ & (1) & C(313) & $\mathrm{H}(310)$ & $3.23(7)$ & (1) \\
\hline$C(313)$ & $\mathrm{H}(311)$ & $2.01(6)$ & (1) & $\mathrm{C}(313)$ & $\mathrm{H}(312)$ & $0.95(2)$ & (1) \\
\hline$C(313)$ & $\mathrm{H}(412)$ & $3.2(4)$ & $(2,1,1,0,-2) \mathrm{C}(3$ & 13) & H(418) & $3.17(5)$ & (1) \\
\hline$C(313)$ & $\mathrm{H}(404)$ & $3.04(4)$ & (1) & $C(313)$ & $\mathrm{H}(207)$ & $3.3(2)$ & $(2,1,1,0,-2)$ \\
\hline$C(516)$ & $\mathrm{H}(515)$ & $2.00(3)$ & (1) & $C(516)$ & $\mathrm{H}(518)$ & $3.26(4)$ & (1) \\
\hline$C(516)$ & $\mathrm{H}(514)$ & $0.95(1)$ & (1) & $C(516)$ & $\mathrm{H}(516)$ & $3.26(5)$ & (1) \\
\hline$C(516)$ & $\mathrm{H}(614)$ & $3.31(9)$ & (1) & $C(516)$ & $\mathrm{H}(503)$ & $2.65(3)$ & (1) \\
\hline$C(516)$ & $\mathrm{H}(603)$ & $3.1(1)$ & (1) & $C(516)$ & $\mathrm{H}(602)$ & $3.16(4)$ & (1) \\
\hline$C(516)$ & $\mathrm{H}(414)$ & $3.4(1)$ & (1) & $C(202)$ & $\mathrm{H}(617)$ & $3.3(9)$ & $(2,1,0,-1,-2)$ \\
\hline$C(202)$ & $\mathrm{H}(209)$ & $2.68(7)$ & (1) & $C(202)$ & $\mathrm{H}(213)$ & $2.65(4)$ & (1) \\
\hline$C(202)$ & $\mathrm{H}(206)$ & $2.67(7)$ & (1) & $C(202)$ & $\mathrm{H}(205)$ & $3.32(6)$ & (1) \\
\hline$C(202)$ & $\mathrm{H}(202)$ & $2.06(4)$ & (1) & $\mathrm{C}(202)$ & $\mathrm{H}(208)$ & $3.23(4)$ & (1) \\
\hline$C(202)$ & $\mathrm{H}(203)$ & $3.26(4)$ & (1) & $C(202)$ & $\mathrm{H}(204)$ & $3.2(1)$ & (1) \\
\hline$C(202)$ & $\mathrm{H}(201)$ & $2.08(4)$ & (1) & $C(205)$ & $\mathrm{H}(312)$ & $3.5(2)$ & $(2,1,1,-1,-2)$ \\
\hline
\end{tabular}




\begin{tabular}{|c|c|c|c|c|c|c|c|}
\hline$C(205)$ & $\mathrm{H}(214)$ & $2.69(8)$ & (1) & $C(205)$ & $\mathrm{H}(218)$ & $2.65(8)$ & (1) \\
\hline$C(205)$ & $\mathrm{H}(205)$ & $3.3(1)$ & (1) & $C(205)$ & $\mathrm{H}(202)$ & $3.2(1)$ & (1) \\
\hline$C(205)$ & $\mathrm{H}(207)$ & $2.75(5)$ & (1) & $C(205)$ & $\mathrm{H}(208)$ & $3.35(6)$ & (1) \\
\hline$C(205)$ & $\mathrm{H}(203)$ & $2.09(3)$ & (1) & $C(205)$ & $\mathrm{H}(204)$ & $2.05(4)$ & (1) \\
\hline$C(205)$ & $\mathrm{H}(201)$ & $3.26(7)$ & (1) & $C(120)$ & $\mathrm{H}(510)$ & $3.4(5)$ & $(1,1,0,0,1)$ \\
\hline$C(120)$ & $\mathrm{H}(318)$ & $3.46(4)$ & (1) & $C(120)$ & $\mathrm{H}(305)$ & $3.6(1)$ & (1) \\
\hline $\mathrm{C}(120)$ & $\mathrm{H}(204)$ & $3.19(4)$ & (1) & $C(120)$ & $\mathrm{H}(114)$ & $3.26(5)$ & (1) \\
\hline$C(120)$ & $\mathrm{H}(116)$ & $3.25(8)$ & (1) & $C(120)$ & $\mathrm{H}(118)$ & $0.95(1)$ & (1) \\
\hline$C(120)$ & $\mathrm{H}(117)$ & $2.01(5)$ & (1) & $C(120)$ & $\mathrm{H}(104)$ & $2.76(4)$ & (1) \\
\hline $\mathrm{C}(420)$ & $\mathrm{H}(316)$ & $2.9(6)$ & $(1,1,1)$ & $C(420)$ & $\mathrm{H}(315)$ & $3.4(2)$ & $(1,1,1)$ \\
\hline C(420) & $\mathrm{H}(416)$ & $3.27(7)$ & (1) & $C(420)$ & $\mathrm{H}(414)$ & $3.23(5)$ & (1) \\
\hline $\mathrm{C}(420)$ & $\mathrm{H}(417)$ & $2.03(4)$ & (1) & $C(420)$ & $\mathrm{H}(418)$ & $0.95(1)$ & (1) \\
\hline C(420) & $\mathrm{H}(404)$ & $2.76(5)$ & (1) & $C(614)$ & H(609) & $3.26(4)$ & (1) \\
\hline C(614) & $\mathrm{H}(612)$ & $2.05(3)$ & (1) & $C(614)$ & $\mathrm{H}(613)$ & $0.95(1)$ & (1) \\
\hline C(614) & $\mathrm{H}(611)$ & 3.3(1) & (1) & $C(614)$ & $\mathrm{H}(602)$ & $2.71(6)$ & (1) \\
\hline$C(614)$ & $\mathrm{H}(108)$ & $3.3(1)$ & $(2,1,0,-1,-2)$ & $C(614)$ & H(106) & $3.2(2)$ & $(2,1,0,-1,-2)$ \\
\hline $\mathrm{C}(320)$ & $\mathrm{H}(607)$ & $3.37(5)$ & (1) & $C(320)$ & $\mathrm{H}(314)$ & $3.26(5)$ & (1) \\
\hline $\mathrm{C}(320)$ & $\mathrm{H}(316)$ & $3.3(2)$ & (1) & $C(320)$ & $\mathrm{H}(318)$ & $0.95(1)$ & (1) \\
\hline $\mathrm{C}(320)$ & $\mathrm{H}(317)$ & $2.01(3)$ & (1) & $C(320)$ & $\mathrm{H}(304)$ & $2.78(8)$ & (1) \\
\hline $\mathrm{C}(320)$ & $\mathrm{H}(217)$ & $3.23(5)$ & (1) & $C(320)$ & $\mathrm{H}(118)$ & $3.59(5)$ & (1) \\
\hline $\mathrm{C}(319)$ & $\mathrm{H}(607)$ & $3.2(1)$ & (1) & $C(319)$ & $\mathrm{H}(316)$ & $2.05(7)$ & (1) \\
\hline $\mathrm{C}(319)$ & $\mathrm{H}(318)$ & $2.02(6)$ & (1) & $C(319)$ & $\mathrm{H}(317)$ & $0.95(2)$ & (1) \\
\hline $\mathrm{C}(319)$ & $\mathrm{H}(315)$ & $3.24(5)$ & (1) & $\mathrm{C}(319)$ & $\mathrm{H}(405)$ & $3.2(2)$ & $(1,1,-1)$ \\
\hline $\mathrm{C}(319)$ & $\mathrm{H}(217)$ & $3.41(8)$ & (1) & $C(204)$ & $\mathrm{H}(314)$ & $3.4(6)$ & $(2,1,0,-1,-2)$ \\
\hline$C(204)$ & $\mathrm{H}(214)$ & $2.81(5)$ & (1) & $C(204)$ & $\mathrm{H}(206)$ & $3.1(1)$ & (1) \\
\hline$C(204)$ & $\mathrm{H}(205)$ & $3.1(2)$ & (1) & $C(204)$ & $\mathrm{H}(202)$ & $2.17(5)$ & (1) \\
\hline$C(204)$ & $\mathrm{H}(207)$ & $2.04(4)$ & (1) & $C(204)$ & $\mathrm{H}(208)$ & $2.04(3)$ & (1) \\
\hline$C(204)$ & $\mathrm{H}(203)$ & $0.95(2)$ & (1) & $C(204)$ & $\mathrm{H}(204)$ & $3.29(4)$ & (1) \\
\hline$C(204)$ & $\mathrm{H}(201)$ & $3.5(1)$ & (1) & $C(110)$ & $\mathrm{H}(608)$ & $3.5(2)$ & $(2,1,0,0,-2)$ \\
\hline$C(110)$ & $\mathrm{H}(113)$ & $3.26(5)$ & (1) & $C(110)$ & $\mathrm{H}(110)$ & $2.02(3)$ & (1) \\
\hline$C(110)$ & $\mathrm{H}(109)$ & $0.95(1)$ & (1) & $C(110)$ & $\mathrm{H}(111)$ & $3.26(5)$ & (1) \\
\hline $\mathrm{C}(110)$ & $\mathrm{H}(101)$ & $2.64(5)$ & (1) & $C(110)$ & $\mathrm{H}(106)$ & $3.2(1)$ & (1) \\
\hline$C(107)$ & $\mathrm{H}(511)$ & $3.4(2)$ & $(1,1,0,0,1)$ & 7) & $\mathrm{H}(606)$ & $2.8(5)$ & $(2,1,0,0,-2)$ \\
\hline$C(107)$ & $\mathrm{H}(109)$ & $3.1(1)$ & (1) & $C(107)$ & $\mathrm{H}(103)$ & $3.34(5)$ & (1) \\
\hline$C(107)$ & $\mathrm{H}(101)$ & $2.07(6)$ & (1) & $C(107)$ & $\mathrm{H}(108)$ & $2.05(3)$ & (1) \\
\hline$C(107)$ & $\mathrm{H}(107)$ & $2.05(6)$ & (1) & $C(107)$ & $\mathrm{H}(105)$ & $0.95(1)$ & (1) \\
\hline$C(107)$ & $\mathrm{H}(106)$ & $0.95(2)$ & (1) & $C(107)$ & $\mathrm{H}(104)$ & $3.08(8)$ & (1) \\
\hline $\mathrm{C}(612)$ & $\mathrm{H}(609)$ & $3.25(7)$ & (1) & $C(612)$ & $\mathrm{H}(612)$ & $2.03(3)$ & (1) \\
\hline$C(612)$ & $\mathrm{H}(610)$ & $2.01(3)$ & (1) & $C(612)$ & $\mathrm{H}(613)$ & 3.3(1) & (1) \\
\hline$C(612)$ & $\mathrm{H}(611)$ & $0.95(1)$ & (1) & $C(612)$ & $\mathrm{H}(617)$ & $3.5(2)$ & $(2,1,0,-1,-3)$ \\
\hline$C(612)$ & $\mathrm{H}(618)$ & $3.1(1)$ & $(2,1,0,-1,-3)$ & $C(612)$ & $\mathrm{H}(416)$ & $3.2(6)$ & $(2,1,1,-1,-3)$ \\
\hline$C(612)$ & $\mathrm{H}(417)$ & $3.6(1)$ & $(2,1,1,-1,-3)$ & $C(612)$ & $\mathrm{H}(206)$ & $3.0(3)$ & $(1,1,0,0,-1)$ \\
\hline$C(612)$ & $\mathrm{H}(108)$ & $2.8(8)$ & $(2,1,0,-1,-2)$ & $C(617)$ & $\mathrm{H}(614)$ & $2.04(2)$ & (1) \\
\hline C(617) & $\mathrm{H}(617)$ & $3.2(1)$ & (1) & $C(617)$ & $\mathrm{H}(616)$ & $2.03(3)$ & (1) \\
\hline$C(617)$ & $\mathrm{H}(615)$ & $0.95(2)$ & (1) & $C(617)$ & $\mathrm{H}(503)$ & $3.3(1)$ & (1) \\
\hline $\mathrm{C}(617)$ & $\mathrm{H}(502)$ & $2.98(5)$ & (1) & $C(617)$ & $\mathrm{H}(314)$ & $3.1(1)$ & (1) \\
\hline $\mathrm{C}(112)$ & $\mathrm{H}(509)$ & $3.2(5)$ & $(1,1,-1,0,1) C$ & & $\mathrm{H}(516)$ & $3.2(4)$ & $(2,1,0,0,-2)$ \\
\hline$C(112)$ & $\mathrm{H}(501)$ & $3.4(6)$ & $(1,1,-1,0,1) C$ & 12) & $\mathrm{H}(306)$ & $3.0(1)$ & $(1,1,-1)$ \\
\hline$C(112)$ & $\mathrm{H}(305)$ & $3.3(8)$ & $(1,1,-1)$ & $C(112)$ & $\mathrm{H}(301)$ & $3.6(1)$ & $(1,1,-1)$ \\
\hline $\mathrm{C}(112)$ & $\mathrm{H}(402)$ & $3.5(3)$ & $(2,1,0,0,-2) C$ & 12) & $\mathrm{H}(113)$ & $3.27(6)$ & (1) \\
\hline $\mathrm{C}(112)$ & $\mathrm{H}(110)$ & $2.00(4)$ & (1) & $C(112)$ & $\mathrm{H}(112)$ & $2.02(4)$ & (1) \\
\hline$C(112)$ & $\mathrm{H}(109)$ & $3.25(5)$ & (1) & $C(112)$ & $\mathrm{H}(111)$ & $0.95(1)$ & (1) \\
\hline C(519) & $\mathrm{H}(513)$ & $3.3(2)$ & $(2,1,1,-1,-3)$ & $C(519)$ & $\mathrm{H}(515)$ & $3.22(5)$ & (1) \\
\hline
\end{tabular}




\begin{tabular}{|c|c|c|c|c|c|c|c|}
\hline$C(519)$ & $\mathrm{H}(518)$ & $2.02(3)$ & (1) & $C(519)$ & $\mathrm{H}(517)$ & $0.95(1)$ & (1) \\
\hline $\mathrm{C}(519)$ & $\mathrm{H}(516)$ & $2.06(3)$ & (1) & $C(519)$ & $\mathrm{H}(613)$ & $3.50(5)$ & (1) \\
\hline $\mathrm{C}(519)$ & $\mathrm{H}(506)$ & $3.4(1)$ & $(2,1,1,-1,-3)$ & $\mathrm{C}(519)$ & $\mathrm{H}(602)$ & $3.4(1)$ & (1) \\
\hline $\mathrm{C}(317)$ & $\mathrm{H}(605)$ & $3.20(5)$ & (1) & $C(317)$ & $\mathrm{H}(607)$ & $2.93(4)$ & (1) \\
\hline $\mathrm{C}(317)$ & $\mathrm{H}(314)$ & $2.05(2)$ & (1) & C(317) & $\mathrm{H}(316)$ & $2.03(3)$ & (1) \\
\hline $\mathrm{C}(317)$ & $\mathrm{H}(317)$ & $3.24(5)$ & (1) & $\mathrm{C}(317)$ & $\mathrm{H}(315)$ & $0.95(1)$ & (1) \\
\hline $\mathrm{C}(317)$ & $\mathrm{H}(207)$ & $3.3(2)$ & $(2,1,0,0,-2) \mathrm{C}$ & 17) & $\mathrm{H}(208)$ & $3.0(3)$ & $(2,1,0,0,-2)$ \\
\hline $\mathrm{C}(317)$ & $\mathrm{H}(203)$ & $3.3(3)$ & $(2,1,0,0,-2) \mathrm{C}$ & 07) & $\mathrm{H}(605)$ & $0.95(1)$ & (1) \\
\hline$C(607)$ & $\mathrm{H}(606)$ & $0.95(1)$ & (1) & $\mathrm{C}(607)$ & $\mathrm{H}(608)$ & $2.08(5)$ & (1) \\
\hline $\mathrm{C}(607)$ & $\mathrm{H}(607)$ & $2.08(5)$ & (1) & $C(607)$ & $\mathrm{H}(603)$ & $3.37(5)$ & (1) \\
\hline $\mathrm{C}(607)$ & $\mathrm{H}(601)$ & $2.09(6)$ & (1) & $C(607)$ & $\mathrm{H}(604)$ & $3.08(5)$ & (1) \\
\hline $\mathrm{C}(607)$ & $\mathrm{H}(216)$ & $3.46(7)$ & (1) & $C(607)$ & $\mathrm{H}(105)$ & $3.3(3)$ & $(2,1,0,-1,-2)$ \\
\hline$C(607)$ & $\mathrm{H}(106)$ & $3.3(7)$ & $(2,1,0,-1,-2)$ & $C(115)$ & $\mathrm{H}(509)$ & $3.5(2)$ & $(1,1,0,0,1)$ \\
\hline$C(115)$ & $\mathrm{H}(510)$ & $3.4(9)$ & $(1,1,0,0,1) \mathrm{C}$ & 15) & $\mathrm{H}(204)$ & $3.12(4)$ & (1) \\
\hline$C(115)$ & $\mathrm{H}(201)$ & $3.57(5)$ & (1) & $\mathrm{C}(115)$ & $\mathrm{H}(114)$ & $2.03(3)$ & (1) \\
\hline$C(115)$ & $\mathrm{H}(118)$ & $2.05(3)$ & (1) & $C(115)$ & $\mathrm{H}(117)$ & $3.3(1)$ & (1) \\
\hline$C(115)$ & $\mathrm{H}(115)$ & $3.3(1)$ & (1) & C(115) & $\mathrm{H}(103)$ & $2.7(1)$ & (1) \\
\hline$C(115)$ & $\mathrm{H}(107)$ & $3.38(7)$ & (1) & $C(115)$ & $\mathrm{H}(104)$ & $2.74(6)$ & (1) \\
\hline C(409) & $\mathrm{H}(409)$ & $2.01(4)$ & (1) & C(409) & $\mathrm{H}(412)$ & $3.28(7)$ & (1) \\
\hline C(409) & $\mathrm{H}(410)$ & $3.26(8)$ & (1) & C(409) & $\mathrm{H}(413)$ & $2.04(4)$ & (1) \\
\hline C(409) & $\mathrm{H}(308)$ & $3.1(1)$ & $(2,1,1,-1,-2)$ & C(409) & $\mathrm{H}(406)$ & $3.45(4)$ & (1) \\
\hline C(409) & $\mathrm{H}(401)$ & $2.72(3)$ & (1) & C(409) & $\mathrm{H}(402)$ & $2.76(4)$ & (1) \\
\hline$C(514)$ & $\mathrm{H}(511)$ & $3.3(1)$ & (1) & $C(514)$ & $\mathrm{H}(512)$ & $2.02(3)$ & (1) \\
\hline$C(514)$ & $\mathrm{H}(509)$ & $3.27(7)$ & (1) & $C(514)$ & $\mathrm{H}(513)$ & $0.95(2)$ & (1) \\
\hline$C(514)$ & $\mathrm{H}(517)$ & $3.0(2)$ & $(2,1,1,0,-3) \mathrm{C}$ & & $\mathrm{H}(516)$ & $3.4(4)$ & $(2,1,1,0,-3)$ \\
\hline$C(514)$ & $\mathrm{H}(610)$ & $3.4(2)$ & $(2,1,0,0,-3) \mathrm{C}$ & 14) & $\mathrm{H}(502)$ & $2.74(7)$ & (1) \\
\hline$C(514)$ & $\mathrm{H}(107)$ & $3.5(2)$ & $(1,1,0,0,-1) \mathrm{C}$ & 08) & $\mathrm{H}(612)$ & $3.0(2)$ & $(2,1,1,0,-3)$ \\
\hline $\mathrm{C}(508)$ & $\mathrm{H}(501)$ & $3.4(1)$ & (1) & $C(508)$ & $\mathrm{H}(506)$ & $2.07(3)$ & (1) \\
\hline$C(508)$ & $\mathrm{H}(505)$ & $2.07(5)$ & (1) & $C(508)$ & $\mathrm{H}(507)$ & $0.95(1)$ & (1) \\
\hline$C(508)$ & $\mathrm{H}(508)$ & $0.95(1)$ & (1) & $C(508)$ & $\mathrm{H}(503)$ & $2.10(6)$ & (1) \\
\hline$C(508)$ & $\mathrm{H}(502)$ & $3.14(5)$ & (1) & $C(508)$ & $\mathrm{H}(211)$ & $3.5(6)$ & $(1,1,1,0,-1)$ \\
\hline $\mathrm{C}(219)$ & $\mathrm{H}(318)$ & $3.30(5)$ & (1) & $\mathrm{C}(219)$ & $\mathrm{H}(317)$ & $3.02(6)$ & (1) \\
\hline$C(219)$ & $\mathrm{H}(412)$ & $2.94(8)$ & (1) & $C(219)$ & $\mathrm{H}(215)$ & $3.2(1)$ & (1) \\
\hline$C(219)$ & $\mathrm{H}(216)$ & $2.01(4)$ & (1) & $C(219)$ & $\mathrm{H}(217)$ & $0.95(2)$ & (1) \\
\hline$C(219)$ & $\mathrm{H}(218)$ & $2.03(3)$ & (1) & $C(502)$ & $\mathrm{H}(509)$ & $2.65(6)$ & (1) \\
\hline$C(502)$ & $\mathrm{H}(513)$ & $2.66(4)$ & (1) & $C(502)$ & $\mathrm{H}(517)$ & $3.5(1)$ & $(2,1,1,0,-3)$ \\
\hline$C(502)$ & $\mathrm{H}(501)$ & $2.07(5)$ & (1) & $C(502)$ & $\mathrm{H}(506)$ & $2.71(4)$ & (1) \\
\hline$C(502)$ & $\mathrm{H}(505)$ & $3.34(6)$ & (1) & $C(502)$ & $\mathrm{H}(508)$ & $3.3(1)$ & (1) \\
\hline$C(502)$ & $\mathrm{H}(503)$ & 3.3(1) & (1) & $C(502)$ & $\mathrm{H}(502)$ & $2.08(3)$ & (1) \\
\hline$C(502)$ & $\mathrm{H}(504)$ & $3.18(4)$ & (1) & C(307) & $\mathrm{H}(413)$ & $3.3(1)$ & $(2,1,1,0,-2)$ \\
\hline C(307) & $\mathrm{H}(308)$ & $2.08(4)$ & (1) & $\mathrm{C}(307)$ & $\mathrm{H}(307)$ & $2.08(5)$ & (1) \\
\hline C(307) & $\mathrm{H}(306)$ & $0.95(1)$ & (1) & C(307) & $\mathrm{H}(305)$ & $0.95(2)$ & (1) \\
\hline C(307) & $\mathrm{H}(301)$ & $2.08(3)$ & (1) & C(307) & $\mathrm{H}(303)$ & $3.4(1)$ & (1) \\
\hline$C(307)$ & $\mathrm{H}(304)$ & $3.13(4)$ & (1) & C(307) & $\mathrm{H}(117)$ & $3.50(6)$ & (1) \\
\hline C(307) & $\mathrm{H}(111)$ & $3.2(1)$ & $(1,1,1)$ & $C(615)$ & $\mathrm{H}(614)$ & $2.04(5)$ & (1) \\
\hline$C(615)$ & $\mathrm{H}(617)$ & $3.27(4)$ & (1) & $\mathrm{C}(615)$ & $\mathrm{H}(618)$ & $2.03(4)$ & (1) \\
\hline$C(615)$ & $\mathrm{H}(615)$ & $3.27(8)$ & (1) & $\mathrm{C}(615)$ & $\mathrm{H}(607)$ & $3.51(5)$ & (1) \\
\hline$C(615)$ & $\mathrm{H}(603)$ & $2.69(4)$ & (1) & $C(615)$ & $\mathrm{H}(604)$ & $2.79(6)$ & (1) \\
\hline C(615) & $\mathrm{H}(502)$ & $3.5(1)$ & (1) & $C(615)$ & $\mathrm{H}(314)$ & $2.99(4)$ & (1) \\
\hline$C(615)$ & $\mathrm{H}(315)$ & $3.2(1)$ & (1) & $\mathrm{C}(201)$ & $\mathrm{H}(209)$ & $2.66(5)$ & (1) \\
\hline$C(201)$ & $\mathrm{H}(206)$ & $2.06(3)$ & (1) & $\mathrm{C}(201)$ & $\mathrm{H}(205)$ & $2.06(3)$ & (1) \\
\hline$C(201)$ & $\mathrm{H}(202)$ & $3.30(9)$ & (1) & $\mathrm{C}(201)$ & $\mathrm{H}(207)$ & $3.13(5)$ & (1) \\
\hline$C(201)$ & $\mathrm{H}(208)$ & $3.16(4)$ & (1) & $\mathrm{C}(201)$ & $\mathrm{H}(203)$ & $3.5(1)$ & (1) \\
\hline
\end{tabular}




\begin{tabular}{|c|c|c|c|c|c|c|c|}
\hline$C(201)$ & $\mathrm{H}(204)$ & $2.15(5)$ & (1) & $\mathrm{C}(201)$ & $\mathrm{H}(201)$ & $0.95(1)$ & (1) \\
\hline$C(201)$ & $\mathrm{H}(114)$ & $3.49(6)$ & (1) & $\mathrm{C}(119)$ & $\mathrm{H}(509)$ & $3.5(5)$ & $(1,1,0,0,1)$ \\
\hline$C(119)$ & $\mathrm{H}(318)$ & $3.4(1)$ & (1) & C(119) & $\mathrm{H}(410)$ & $3.45(7)$ & (1) \\
\hline C(119) & $\mathrm{H}(411)$ & $3.49(6)$ & (1) & C(119) & $\mathrm{H}(305)$ & $3.28(6)$ & (1) \\
\hline C(119) & $\mathrm{H}(304)$ & $3.3(1)$ & (1) & C(119) & $\mathrm{H}(218)$ & $3.37(7)$ & (1) \\
\hline C(119) & $\mathrm{H}(204)$ & $3.20(5)$ & (1) & $\mathrm{C}(119)$ & $\mathrm{H}(116)$ & $2.05(5)$ & (1) \\
\hline C(119) & $\mathrm{H}(118)$ & $2.01(4)$ & (1) & $\mathrm{C}(119)$ & $\mathrm{H}(117)$ & $0.95(2)$ & (1) \\
\hline C(119) & $\mathrm{H}(115)$ & $3.28(6)$ & (1) & C(119) & $\mathrm{H}(112)$ & $3.5(4)$ & $(1,1,1)$ \\
\hline$C(506)$ & $\mathrm{H}(518)$ & $2.72(4)$ & (1) & $\mathrm{C}(506)$ & $\mathrm{H}(501)$ & $2.07(3)$ & (1) \\
\hline$C(506)$ & $\mathrm{H}(506)$ & $3.33(4)$ & (1) & $\mathrm{C}(506)$ & $\mathrm{H}(505)$ & $2.76(4)$ & (1) \\
\hline$C(506)$ & $\mathrm{H}(507)$ & $3.2(1)$ & (1) & $\mathrm{C}(506)$ & $\mathrm{H}(503)$ & $3.25(5)$ & (1) \\
\hline$C(506)$ & $\mathrm{H}(504)$ & $0.95(1)$ & (1) & $C(506)$ & $\mathrm{H}(212)$ & $3.4(5)$ & $(1,1,1,0,-1)$ \\
\hline$C(506)$ & $\mathrm{H}(115)$ & $3.2(1)$ & $(1,1,0,0,-1) C$ & 12) & $\mathrm{H}(505)$ & $3.3(6)$ & $(1,1,-1,0,1)$ \\
\hline$C(212)$ & $\mathrm{H}(415)$ & $2.9(6)$ & $(1,1,-1,0,1)$ & 12) & $\mathrm{H}(416)$ & $3.5(7)$ & $(1,1,-1,0,1)$ \\
\hline$C(212)$ & $\mathrm{H}(212)$ & $2.04(6)$ & (1) & $C(212)$ & $\mathrm{H}(209)$ & $3.27(8)$ & (1) \\
\hline$C(212)$ & $\mathrm{H}(210)$ & $2.04(6)$ & (1) & $C(212)$ & $\mathrm{H}(211)$ & $0.95(2)$ & (1) \\
\hline$C(212)$ & $\mathrm{H}(213)$ & $3.24(6)$ & (1) & $C(212)$ & $\mathrm{H}(102)$ & $3.07(6)$ & (1) \\
\hline$C(512)$ & $\mathrm{H}(511)$ & $0.95(2)$ & (1) & $C(512)$ & $\mathrm{H}(512)$ & $2.04(3)$ & (1) \\
\hline$C(512)$ & $\mathrm{H}(509)$ & $3.28(5)$ & (1) & $\mathrm{C}(512)$ & $\mathrm{H}(510)$ & $2.03(4)$ & (1) \\
\hline$C(512)$ & $\mathrm{H}(513)$ & $3.3(1)$ & (1) & $C(512)$ & $\mathrm{H}(414)$ & $2.9(1)$ & $(2,1,1,0,-3)$ \\
\hline$C(512)$ & $\mathrm{H}(403)$ & $2.9(2)$ & $(2,1,1,0,-3)$ & 12) & $\mathrm{H}(107)$ & $3.0(1)$ & $(1,1,0,0,-1)$ \\
\hline$C(512)$ & $\mathrm{H}(105)$ & $2.8(1)$ & $(1,1,0,0,-1)$ & 14) & $\mathrm{H}(617)$ & $3.5(2)$ & $(2,1,0,-1,-2)$ \\
\hline$C(214)$ & $\mathrm{H}(616)$ & $2.9(2)$ & $(2,1,0,-1,-2)$ & $C(214)$ & $H(212)$ & $2.02(5)$ & (1) \\
\hline$C(214)$ & H(209) & $3.3(1)$ & (1) & $C(214)$ & $\mathrm{H}(211)$ & $3.25(6)$ & (1) \\
\hline$C(214)$ & $\mathrm{H}(213)$ & $0.95(2)$ & (1) & $C(214)$ & $\mathrm{H}(202)$ & $2.79(5)$ & (1) \\
\hline$C(214)$ & $\mathrm{H}(102)$ & $3.16(4)$ & (1) & $C(608)$ & $\mathrm{H}(605)$ & $2.08(3)$ & (1) \\
\hline$C(608)$ & $\mathrm{H}(606)$ & $2.08(3)$ & (1) & $C(608)$ & $\mathrm{H}(608)$ & $0.95(1)$ & (1) \\
\hline$C(608)$ & $\mathrm{H}(607)$ & $0.95(1)$ & (1) & $\mathrm{C}(608)$ & $\mathrm{H}(603)$ & $2.10(5)$ & (1) \\
\hline$C(608)$ & $\mathrm{H}(601)$ & $3.4(2)$ & (1) & $\mathrm{C}(608)$ & $\mathrm{H}(602)$ & $3.1(1)$ & (1) \\
\hline$C(608)$ & $\mathrm{H}(216)$ & $3.37(8)$ & (1) & $C(518)$ & $\mathrm{H}(515)$ & $2.00(3)$ & (1) \\
\hline$C(518)$ & $\mathrm{H}(518)$ & $3.26(5)$ & (1) & $C(518)$ & $\mathrm{H}(514)$ & $3.25(4)$ & (1) \\
\hline$C(518)$ & $\mathrm{H}(517)$ & $2.04(3)$ & (1) & $\mathrm{C}(518)$ & $\mathrm{H}(516)$ & $0.95(1)$ & (1) \\
\hline$C(518)$ & $\mathrm{H}(506)$ & $3.5(2)$ & $(2,1,1,-1,-3)$ & $C(518)$ & $\mathrm{H}(602)$ & $3.16(4)$ & (1) \\
\hline$C(518)$ & $\mathrm{H}(414)$ & $3.35(9)$ & (1) & $C(518)$ & $\mathrm{H}(110)$ & $3.5(4)$ & $(2,1,0,-1,-2)$ \\
\hline$C(117)$ & $\mathrm{H}(509)$ & $2.9(6)$ & $(1,1,0,0,1)$ & 17) & $\mathrm{H}(501)$ & $3.6(1)$ & $(1,1,0,0,1)$ \\
\hline$C(117)$ & $\mathrm{H}(204)$ & $3.1(1)$ & (1) & $\mathrm{C}(117)$ & $\mathrm{H}(201)$ & $3.24(5)$ & (1) \\
\hline$C(117)$ & $\mathrm{H}(114)$ & $2.04(5)$ & (1) & $\mathrm{C}(117)$ & $\mathrm{H}(116)$ & $2.05(6)$ & (1) \\
\hline$C(117)$ & $\mathrm{H}(117)$ & $3.28(5)$ & (1) & $\mathrm{C}(117)$ & $\mathrm{H}(115)$ & $0.95(1)$ & (1) \\
\hline$C(501)$ & $\mathrm{H}(509)$ & $2.8(1)$ & (1) & $C(501)$ & $\mathrm{H}(501)$ & $0.95(1)$ & (1) \\
\hline$C(501)$ & $\mathrm{H}(506)$ & $2.07(3)$ & (1) & $C(501)$ & $\mathrm{H}(505)$ & $2.08(3)$ & (1) \\
\hline $\mathrm{C}(501)$ & $\mathrm{H}(507)$ & $3.1(2)$ & (1) & $\mathrm{C}(501)$ & $\mathrm{H}(508)$ & $3.2(1)$ & (1) \\
\hline$C(501)$ & $\mathrm{H}(503)$ & $3.55(5)$ & (1) & $C(501)$ & $\mathrm{H}(502)$ & $3.3(1)$ & (1) \\
\hline$C(501)$ & $\mathrm{H}(504)$ & $2.16(3)$ & (1) & $C(501)$ & $\mathrm{H}(112)$ & $3.5(6)$ & $(1,1,1,0,-1)$ \\
\hline$C(504)$ & $\mathrm{H}(514)$ & $2.73(4)$ & (1) & $C(504)$ & $\mathrm{H}(501)$ & $3.55(5)$ & (1) \\
\hline$C(504)$ & $\mathrm{H}(506)$ & $3.15(9)$ & (1) & $C(504)$ & $\mathrm{H}(505)$ & $3.2(1)$ & (1) \\
\hline$C(504)$ & $\mathrm{H}(507)$ & $2.06(3)$ & (1) & $C(504)$ & $\mathrm{H}(508)$ & $2.06(3)$ & (1) \\
\hline$C(504)$ & $\mathrm{H}(503)$ & $0.95(1)$ & (1) & $\mathrm{C}(504)$ & $\mathrm{H}(502)$ & $2.16(3)$ & (1) \\
\hline$C(504)$ & $\mathrm{H}(504)$ & $3.29(4)$ & (1) & $C(220)$ & $\mathrm{H}(312)$ & $3.5(9)$ & $(2,1,1,-1,-2)$ \\
\hline$C(220)$ & $\mathrm{H}(318)$ & $3.52(6)$ & (1) & $C(220)$ & $\mathrm{H}(317)$ & $3.34(9)$ & (1) \\
\hline$C(220)$ & $\mathrm{H}(412)$ & $3.27(7)$ & (1) & $C(220)$ & $\mathrm{H}(411)$ & $3.4(1)$ & (1) \\
\hline$C(220)$ & $\mathrm{H}(214)$ & $3.26(5)$ & (1) & $\mathrm{C}(220)$ & $H(216)$ & $3.23(6)$ & (1) \\
\hline$C(220)$ & $\mathrm{H}(217)$ & $2.02(6)$ & (1) & $C(220)$ & $\mathrm{H}(218)$ & $0.95(2)$ & (1) \\
\hline$C(220)$ & $\mathrm{H}(204)$ & $2.75(4)$ & (1) & $\mathrm{C}(609)$ & $\mathrm{H}(609)$ & $2.04(3)$ & (1) \\
\hline
\end{tabular}




$\begin{array}{llllllll}\mathrm{C}(609) & \mathrm{H}(612) & 3.30(7) & (1) & \mathrm{C}(609) & \mathrm{H}(610) & 3.3(1) & (1) \\ \mathrm{C}(609) & \mathrm{H}(613) & 2.04(2) & (1) & \mathrm{C}(609) & \mathrm{H}(606) & 3.50(8) & (1) \\ \mathrm{C}(609) & \mathrm{H}(601) & 2.72(7) & (1) & \mathrm{C}(609) & \mathrm{H}(602) & 2.74(8) & (1) \\ \mathrm{C}(609) & \mathrm{H}(108) & 3.4(1) & (2,1,0,-1,-2) & \mathrm{C}(609) & \mathrm{H}(106) & 2.9(1) & (2,1,0,-1,-2) \\ \mathrm{C}(513) & \mathrm{H}(511) & 2.06(5) & (1) & \mathrm{C}(513) & \mathrm{H}(512) & 0.95(2) & (1) \\ \mathrm{C}(513) & \mathrm{H}(510) & 3.26(5) & (1) & \mathrm{C}(513) & \mathrm{H}(513) & 2.03(5) & (1) \\ \mathrm{C}(513) & \mathrm{H}(609) & 3.0(3) & (2,1,0,0,-3) \mathrm{C}(513) & \mathrm{H}(610) & 3.4(3) & (2,1,0,0,-3) \\ \mathrm{C}(513) & \mathrm{H}(415) & 3.5(8) & (2,1,1,0,-3) \mathrm{C}(513) & \mathrm{H}(414) & 3.1(1) & (2,1,1,0,-3) \\ \mathrm{C}(513) & \mathrm{H}(107) & 3.2(1) & (1,1,0,0,-1) & & & & \end{array}$

*ADC (S, L, TX, TY, TZ) represents:

S: Symmetry operation number. $-\mathrm{S}$ is inversion of operation $\mathrm{S}$.

(1) $X, Y, Z$

(2) $-X, Y+1 / 2,-Z$

L: Lattice translation.

(1) $0,0,0$

TX, TY, TZ: Unit cell translation along the $\mathrm{x}, \mathrm{y}$, and $\mathrm{z}$ directions. 